\title{
Ethics at the interface of reproductive medicine and genetic technology : the case of mitochondrial disorders
}

Citation for published version (APA):

Bredenoord, A. L. (2010). Ethics at the interface of reproductive medicine and genetic technology : the case of mitochondrial disorders. [Doctoral Thesis, Maastricht University]. Maastricht University. https://doi.org/10.26481/dis.20100618ab

Document status and date:

Published: 01/01/2010

DOI:

10.26481/dis.20100618ab

Document Version:

Publisher's PDF, also known as Version of record

Please check the document version of this publication:

- A submitted manuscript is the version of the article upon submission and before peer-review. There can be important differences between the submitted version and the official published version of record.

People interested in the research are advised to contact the author for the final version of the publication, or visit the DOI to the publisher's website.

- The final author version and the galley proof are versions of the publication after peer review.

- The final published version features the final layout of the paper including the volume, issue and page numbers.

Link to publication

\footnotetext{
General rights rights.

- You may freely distribute the URL identifying the publication in the public portal. please follow below link for the End User Agreement:

www.umlib.nl/taverne-license

Take down policy

If you believe that this document breaches copyright please contact us at:

repository@maastrichtuniversity.nl

providing details and we will investigate your claim.
}

Copyright and moral rights for the publications made accessible in the public portal are retained by the authors and/or other copyright owners and it is a condition of accessing publications that users recognise and abide by the legal requirements associated with these

- Users may download and print one copy of any publication from the public portal for the purpose of private study or research.

- You may not further distribute the material or use it for any profit-making activity or commercial gain

If the publication is distributed under the terms of Article $25 \mathrm{fa}$ of the Dutch Copyright Act, indicated by the "Taverne" license above, 
Ethics at the interface of reproductive medicine and genetic technology: the case of mitochondrial disorders

Annelien Bredenoord 
Maastricht University, Faculty of Health, Medicine and Life Sciences

Thesis Maastricht University - with a summary in Dutch

Proefschrift Universiteit Maastricht - met een samenvatting in het Nederlands

ISBN/EAN: $\quad$ 978-90-5335-283-0

Author: Annelien Bredenoord

Cover design: $\quad$ Simone Vinke, Ridderprint BV

Cover pictures: Salvador Dali, DNA Structure.

Lay-out: Annelien Bredenoord

Printed by: $\quad$ Ridderprint BV, Ridderkerk, Netherlands

Financial support by the J.E. Jurriaanse Stichting for the publication of this thesis were gratefully acknowledged. 
Ethics at the interface of reproductive medicine and genetic technology: the case of mitochondrial disorders

\section{PROEFSCHRIFT}

ter verkrijging van de graad van doctor aan de Universiteit Maastricht, op gezag van de Rector Magnificus, Prof Mr G.P.M.F. Mols

volgens het besluit van het College van Decanen,

in het openbaar te verdedigen

op vrijdag 18 juni 2010 om 12.00 uur

door

Anne Lydia Bredenoord 


\section{Promotores:}

Prof. dr. G. de Wert

Prof. dr. G. Pennings (Ghent University, Belgium)

\section{Copromotor:}

Dr. W.J. Dondorp

\section{Beoordelingscommissie:}

Prof. dr. J. Geraedts (chair)

Prof. dr. I.D. de Beaufort (Erasmus University Rotterdam)

Prof. dr. ir. W. Bijker

Prof. dr. J.L.H. Evers

Prof. dr. J. Poulton (Oxford University, United Kingdom) 


\section{Contents}

Chapter 1 General introduction

Chapter 2 Dealing with uncertainties:

ethics of prenatal diagnosis and preimplantation genetic diagnosis to prevent mitochondrial disorders Human Reproduction Update 2008;14(1):83-94

Chapter 3 PGD to reduce reproductive risk:

the case of mitochondrial DNA disorders

Human Reproduction 2008;23(11):2392-401

Chapter 4 Preimplantation genetic diagnosis for

mitochondrial DNA disorders: ethical guidance

for clinical practice

European Journal of Human Genetics 2009;17(12):1550-1559

Chapter 5 Reproductive decision-making in the context of mitochondrial DNA disorders: views and experiences of professionals

Clinical Genetics 2010;77(1):10-17

Chapter 6 Ooplasmic and nuclear transfer to prevent mitochondrial DNA disorders: conceptual and normative issues Human Reproduction Update 2008;14(6):669-678

Chapter 7 Ethics of nuclear transfer to prevent mitochondrial DNA disorders: revisiting the debate on germ-line modification and reproductive cloning Submitted for publication

Chapter 8 Avoiding transgenerational risks of mitochondrial DNA disorders: a morally acceptable reason for sex selection? Human Reproduction, in press 
Chapter 9 General Discussion

References

173

Summary

199

Samenvatting

209

Dankwoord

221

Curriculum Vitae

227 
Chapter 1

General Introduction 



\subsection{The interface of assisted reproductive technology and genetics}

This thesis examines ethical issues that arise at the interface of assisted reproductive technology (ART) and genetics. At this crossroads of reproductive genetic technologies, questions regarding, for instance, embryo research and testing, oocyte donation, the welfare of future children, professional responsibilities, reproductive autonomy, germ-line modification and reproductive cloning not only confront parentsto-be and health care professionals, but also society and politics at large.

More in particular, this thesis examines a specific case: the ethical issues that arise when reproductive genetic technology is used to prevent the transmission of mitochondrial disorders, particularly those caused by a mutation in the mitochondrial DNA (mtDNA). Mitochondrial disorders are clinically heterogeneous but usually severe disorders, involving defects in energy production. Whereas the ethical issues at the crossroads of ART and genetics are complex, this is even more so when reproductive genetic technology is deployed in the context of disorders caused by a mutation in the mtDNA. This is mainly due to the specific characteristics of mitochondrial genetics, which differs considerably from Mendelian genetics (Tuppen et al, 2009). This thesis offers an ethical evaluation of various reproductive genetic technologies used with the aim of preventing the transmission of mitochondrial DNA disease.

The central topic of this thesis could be interpreted and localized in a wider debate regarding the acceptability of novel (applications of) ART, not primarily used to overcome infertility problems, but to prevent genetic disease. ART was initially introduced as a form of treatment for couples facing infertility. Louise Brown, born in 1978, was the first baby conceived by in vitro fertilisation (IVF). Whereas ART was initially used for infertility problems, it has increasingly been deployed as a vehicle for genetic technology. An outstanding present-day example of this alliance between ART and genetics is preimplantation genetic diagnosis (PGD). PGD can be perceived as an early form of prenatal diagnosis (PND), in which embryos created in vitro are analysed for a particular genetic mutation. After the genetic analysis, one or two unaffected embryos are transferred to the womb (Sermon et al, 2004; de Wert, 2005; Geraedts and de Wert, 2009; Harper, 2009). Clearly, PGD usually presupposes IVF; in this context, IVF is used to produce embryos that can be subjected to genetic analysis. As UNESCO has put it: 'IVF aims at having a child, PGD aims at having a healthy child' (UNESCO, 2003; Soini et al, 2006). A possible future example of the alliance between ART and genetics is the use of nuclear transfer techniques - the transfer of a nucleus of an oocyte or early embryo to an enucleated donor oocyte. Nuclear transfer became well known because it was used to create the sheep Dolly (Campbell et al, 1996), but its use in humans with the aim 
of preventing mitochondrial genetic disease is announced as well (e.g. Tachibana et al, 2009).

The emergence of reproductive genetic technology has provided couples, who were previously anxious to have children because they or their family members were affected by or carrier of a genetic disorder, with several reproductive options. At the same time, future parents, professionals and society have to find a way to deal with the complex ethical questions evoked by reproductive genetic technology. This thesis aims to contribute to the ethical debate regarding the acceptability of novel (applications of existing) reproductive genetic technology by examining the case of mtDNA disorders. Addressed are both the ethical issues 'at the bench', when a reproductive genetic technology is preclinically (in the laboratory) developed as well as the ethical issues 'at the bedside', when a reproductive genetic technology is offered to patients in the clinic.

\subsection{Mitochondrial disorders}

Although mitochondrial disease may present 'with any symptom in any organ at any age' (Munnich et al, 1996), often the most energy demanding tissues such as the central nervous system, heart and skeletal muscles, liver and kidneys are affected. As a consequence of this variable phenotype, patients are seen by a variety of clinical specialists. Symptoms may vary from exercise intolerance in older patients up to fatal multisystem disease with an onset in early childhood. Due to the variability in clinical symptoms, the diagnosis of mitochondrial disease is not easily made and the prevalence of mitochondrial disease is difficult to establish (McFarland et al, 2007). Recent studies indicate with regard to disorders due to a defect in the mtDNA that about 1 in 5000 people have, or are likely to develop mtDNA disease (Schaefer et al, 2008) and as many as 1 in 200 newborns carry one of the common mtDNA mutations (Elliott et al, 2008), making mtDNA disease one of the most common inherited neuromuscular disorders.

The mitochondrial genome is not well-known. When people talk about genes, they usually refer to the nuclear genome. All human cells, however, contain two different genomes. One indeed is located in the nucleus and follows a Mendelian pattern of autosomal recessive, dominant or X-linked inheritance. This genome is by far the largest, containing about $22.000-24.000$ genes. The other genome is located in the mitochondria. These are tiny organelles in the cytoplasma surrounding the nucleus; they serve as the 'powerhouses' of the cell. The mitochondrial genome is extremely small, containing 37 genes, which is about $0.1 \%$ of the total amount of DNA (Taylor 
and Turnbull, 2005). The relevant genes are all involved in the cell's energy metabolism, whereas the nuclear DNA has a much wider range of functions.

Mitochondria are under the dual genetic control of both the nuclear and mitochondrial genomes (McFarland et al, 2007). Mitochondrial genetics varies from Mendelian genetics in several aspects (Taylor and Turnbull, 2005). As a consequence, diseases due to a defect in the mtDNA show some special characteristics. Firstly, many mtDNA mutations are heteroplasmic. This means that there is a mixture of normal and mutant mtDNA in one person, the level of which can differ among tissues. If the mutant load (the ratio of mutant to normal mtDNA) exceeds a specific threshold, clinical features become manifest. However, the exact threshold to disease expression is often not known and genotype-phenotype correlations are often lacking, even within families. Secondly, the mtDNA is maternally inherited. Due to the so-called genetic bottleneck, extreme shifts in mutant load can be observed between mother and child and also siblings can have variable levels of mutant load (Marchington et al, 2009). These specifics of mtDNA genetics are further elaborated in chapter 2.

\subsection{Aims and scope of this thesis}

As there is no curative treatment, helping carriers of mitochondrial mutations to have healthy children has been a central focus of attention (Taylor and Turnbull, 2005; Chinnery et al, 2006). A distinction can be made between strategies that do not, or only partly provide parents with a genetically related child, and reproductive strategies that fully maintain the genetic link to both parents. The first group, which includes adoption and oocyte donation, is only mentioned briefly in this thesis. The focus is on the second group of reproductive options. This group can be divided in two types of interventions:

\section{(1) Genetic testing and selection}

a. Prenatal diagnosis (PND): the genetic testing of a fetus, either by means of chorionic villus sampling (CVS, around week 12 of gestation), or by means of amniocentesis (usually in week 14-18 of gestation).

b. Preimplantation genetic diagnosis (PGD): PGD presupposes in vitro fertilization (IVF) with or without intracytoplasmic sperm injection (ICSI). One or two cells are dissected for genetic analysis. This usually happens at the eight-cell stage. After the genetic analysis, only the unaffected embryos will be transferred. An early variant of PGD regards polar body analysis, in which the first (and possibly also second) polar body of the oocyte is used for the genetic analysis. 
(2) Genetic intervention or modification

a. Ooplasmic transfer (OT): the transfer of donor ooplasm with normal mitochondria to the oocyte of a woman who carries mutant mtDNA in her oocytes.

b. Mitochondrial gene replacement by means of nuclear transfer (NT): the transfer of the nuclear DNA of a woman carrying an mtDNA mutation into an enucleated donor oocyte. The nuclear transplantation can be performed before and after IVF, using the nucleus of an unfertilized oocyte, the pronuclei of the zygote or the nucleus of a blastomere of an embryo.

The main goal of this thesis is to ethically evaluate the above strategies to prevent the transmission of mitochondrial disease, particularly those caused by a mutation in the mtDNA. Whereas some of the above strategies are already - albeit cautiously offered in clinical practice (PND, PGD), other strategies are at the moment (still) theoretical (NT). Nevertheless, as preclinical experiments to examine the feasibility of NT are currently conducted, a pro-active ethical analysis is of paramount importance. Subgoals of this thesis are to make an inventory of the ethical issues involved in these various reproductive strategies and, where possible and appropriate, to develop ethical guidelines for their preclinical development and clinical application.

The main research question is: how should we ethically evaluate the various reproductive strategies for couples at risk of transmitting a mitochondrial disorder to their offspring, particularly for carriers of mtDNA mutations? Whereas PND, PGD, OT as well as NT for mtDNA disorders will be discussed, special attention is given to PGD and NT.

\subsection{Structure of this thesis}

The main chapters of this thesis consist of articles, which have been published or submitted for publication.

Chapters 2, 3 and 4 discuss the reproductive options that entail genetic selection of the embryo or fetus. Chapter 2 offers an overview of the moral landscape of PND and PGD for mitochondrial disorders. First, a distinction is made between mitochondrial disorders caused by a mutation in the nuclear DNA, mitochondrial disorders with an unknown genetic cause and mitochondrial disorders caused by a mutation in the mtDNA, the so-called 'mtDNA disorders'. Second, a classification is made of different types of mtDNA mutations. Subsequently, an inventory is made of the ethical issues involved in PND and PGD for mtDNA disease. What about the ethics of offering suboptimal reproductive genetic tests, in the sense that the results 
may be difficult to interpret and not very predictive? Is it acceptable to terminate a pregnancy based on ambiguous test results? How to obtain informed consent?

Chapter 3 goes more deeply into the subject of PGD for mtDNA disorders. Whereas more 'traditional' uses of PGD are targeted at transferring embryos that are free of the mutation in question, it is conceivable that in PGD for mtDNA disorders the best one can achieve is to transfer the least affected embryo. Would such a 'risk-reducing PGD' be morally acceptable? Is it acceptable to (knowingly) bring a child into the world whose health may be impaired? What about parental and professional responsibilities? In this chapter, we defend the reasonable welfare standard, which is the view that for (medically assisted) reproduction to be justified, the future child must have a reasonable chance of an acceptable quality of life. The contra-indication is a high risk of serious harm. In addition, the medical team should make efforts to reduce the risks as much as reasonably possible. We conclude that although introducing such an application of PGD in clinical practice would make high demands on the medical team, no convincing arguments exist to regard this risk-reducing PGD morally unjustified beforehand.

Nevertheless, several ethical questions arise when considering application in clinical practice. Is it morally justified to (experimentally) offer PGD, or is this still premature? How to determine a cut-off point below which embryos are considered to be eligible for transfer? To what extent should one search for the best possible embryo? These and other questions are addressed in chapter 4, in which the conditions for a responsible introduction and use of PGD for mtDNA disorders in clinical practice are examined.

Chapter 5 is based on the semi-structured interviews that have been conducted during the research project resulting in this thesis. It explores the attitudes and experiences of professionals involved on a daily basis with their patients' reproductive decision-making in the context of mtDNA disease. As professionals feel that the inherent challenges of reproductive genetic testing and counseling are intensified in the context of mtDNA disorders, this chapter unfolds the tension between imperfect knowledge - inherent to the rapid developments in medical genetics and the application of state of the art science - and clinical practice.

Chapters 6 and 7 examine the reproductive options that entail genetic intervention in the oocyte or (early) embryo. Chapter 6 offers an overview of the conceptual and moral landscape surrounding OT and NT to prevent mtDNA disorders. Should we consider OT and NT as types of germ-line modification, and if so, what about the ethics? Is NT a type of human cloning? What about the health and safety risks of OT and NT? NT results in a child carrying its nuclear DNA from its parents and the mtDNA from an oocyte donor. Should we consider the oocyte donor a genetic parent 
and if so, what about the moral implications of having 'three genetic parents'? Although it is by now generally accepted that OT is not a suitable option to prevent the transmission of a mitochondrial mutation, an inventory of the moral issues involved is nevertheless of relevance, also in light of its (according to many premature) use as an infertility technique and the ongoing preclinical experiments.

Chapter 7 goes more deeply into the subject of NT to prevent mtDNA disease. First, we argue that whereas all variants of NT entail germ-line modification, one variant also implies reproductive cloning $(\mathrm{RC})$. Second, we examine the validity of the main categorical (non-safety) arguments used against germ-line modification and RC. Opponents have for instance argued that germ-line modification and $R C$ are repugnant and unnatural, or that they violate human dignity, or that they violate the child's right to an open future. What about the validity of these objections? And do they apply in the context of NT to prevent mtDNA disease?

Chapter 8 discusses the acceptability of 'transgenerational' sex selection: not intended to avoid the birth of a child with a severe genetic disorder, but to avoid health risks further along the line of generations. Whereas sex selection is highly controversial for nonmedical uses, it is generally accepted for medical reasons. The use as described in this chapter entails an intermediate one: although it would not fall within the bounds of strict medical applications of sex selection (avoiding a child with a severe genetic disorder), neither does it seem correct to present it as an instance of sex selection for nonmedical reasons. The reason for bringing up this issue is that sex selection may be a helpful additional measure in the context of preventing mtDNA disease. In a clinical application of PGD and in a possible future application of NT for mtDNA disorders, the resulting child may still carry a certain amount of mutant mtDNA. Although this mutant load is expected to be below the threshold for disease expression, the mutant load may rise in the third generation - the couple's grandchildren. As mitochondria are transferred maternally, male offspring will not pass on their mutant mtDNA to the next generation. Does the avoidance of transgenerational health risks provide a morally acceptable reason for sex selection?

Finally, chapter 9 looks back at the main results, conclusions and limitations of this study. It looks forward as well, to questions for future research.

\subsection{Methods: Wide Reflective Equilibrium}

This thesis is based on the Workpackage Ethics of the European Union $6^{\text {th }}$ Framework Program MITOCIRCLE: "Mitochondrial diseases: from bedside to genome to bedside". In this interdisciplinary project, researchers and clinicians from 5 leading centers from 4 European countries (Italy, France, United Kingdom and the 
Netherlands) participated in order to optimize efficient and accurate diagnosis throughout the EU and thereby establish best practice for the clinical investigation and management of patients and families with mitochondrial disease. This setting provided me with an excellent opportunity to closely collaborate with clinicians and researchers at the bench. In this thesis, extensive attention has been paid to (clinical and research) practice by means of working visits to the participating centers and by means of semi-structured interviews with patients, health care professionals and policy-makers. Adding intuitions and experiences from practitioners to ethics research offers an invaluable supplementation and enrichment of the scientific and scholarly debates, literature and theory. The alliance with practice is a prerequisite for practicing ethics well-informed and pro-actively and for avoiding armchair philosophy.

This attention to practice however raises questions about how these intuitions and experiences from clinical practice can be integrated in ethical theory and analysis. After all, although intuitions and experiences are highly valuable as moral markers, the added value of ethics is to reflect, rethink and systematically analyze those intuitions and experiences in order to decide whether they indeed are right or wrong, and to arrive at a justifiable moral judgment or theory. Our intuitions and experiences may be misleading and put us in the wrong direction. Intuition and experience should be subjected to rational analysis, because otherwise it would imply a permit for moral prejudice (Nussbaum, 2004). Gillon (1999) for instance illustrates this with an example from medicine: whereas in daily life it is (generally speaking) a rather strange thing to put your finger in a stranger's bottom, in medical practice it actually can be the right thing to do. Moreover, not doing it can sometimes even be considered negligence. In other words, careful moral analysis may show that things are not always as straightforward as they seem to be or vice versa.

Wide Reflective Equilibrium (RE) is a model for moral reasoning. It was introduced by John Rawls in 1971, in his seminal work $A$ Theory of Justice, as an argumentative method for developing and justifying a theory of justice (Rawls, 1971). Many others have adjusted and modified the concept of RE after Rawls. Particularly in the context of applied ethics, it is often used as a method to integrate practical experience, intuitions and empirical data with ethical theory.

A RE is both a reasoning process and the product resulting from this process, although the product is always a 'provisional fixed point', not an endpoint (Rawls, 1971; Van der Burg and Van Willigenburg, 1998). RE is a procedure of adjusting and rendering intuitions, principles, morally relevant facts and relevant background theories into a coherent moral view. This process can be characterized as a dialogue 
of going back and forth between beliefs stemming from practice (morally relevant facts and moral intuitions) and from theory (principles and background theory) (Van Delden and Van Thiel, 1998). From the perspective of RE, ethical reasoning is analogous to hypotheses in science: in a continuous process we test them, modify them, refine them or reject them through experiments and experimental thinking (Beauchamp and Childress, 2009). As the outcomes are provisional fixed points, both science and ethics are never finished for they require continuous refinement and adjustment. They should be considered work in progress rather than finished products (Beauchamp and Childress, 2009).

In recent years, ethicists increasingly began to incorporate empirical research (using methods from social science) in the model of RE. The empirical data are used as input for the reasoning process. These data are of different nature and can play several roles in the reasoning process. One of the roles is to provide the ethicist with knowledge about views, experiences and attitudes. The ethicist is taken to integrate this enriched set of relevant facts and beliefs in a process of reasoning towards a morally justifiable conclusion. In this study, the views, experiences and attitudes of professionals, patients and policy-makers were examined by means of a qualitative interview design (Patton, 2002). Whereas only chapter 5 is explicitly based on the interviews with professionals, the information obtained in the interviews is more implicitly interwoven in the other chapters as well.

Next to these empirical data, I used casuistry - but not as a method to reach moral conclusions (Jonsen and Toulmin, 1988; Beauchamp and Childress, 2009), but as a heuristic tool to explore new cases. By means of case-comparison, precedent cases and analogy, novel cases can be placed in the context of similar cases. By exploring the morally relevant similarities and differences, the morally relevant features of a case can be elucidated. This vividly illustrates the case under study, enables understanding and enriches the moral reasoning process. In chapter 8 for example we elaborate the analogy of sex selection in the context of preventing mtDNA disorders compared to sex selection in the context of preventing $X$-linked disease with the aim to clarify the characteristics of the case in question. As casuistry is a method without content (Beauchamp and Childress, 2009), it is a useful tool of thought for case-comparison and analogous thinking, but not suitable in itself as a method of justification. Therefore it needs to be supplemented with argumentation, principles and critical analysis.

In ethics, the ultimate objective is to establish one's case by presenting sufficient grounds for it (Beauchamp and Childress, 2009). In other words, what is needed is 
moral justification. Whether this thesis succeeded in providing these sufficient and convincing grounds is up to the reader.

In his essay The Future of Human Nature, the German philosopher Habermas (2003) makes an interesting remark. Whereas in Germany the bioethical discussion is targeted at the question of whether further developments in genetic technology are desirable and permissible, the debate in the USA focuses on the question of how the developments in genetic technology should be implemented. This distinction is a well-known one in medical ethics. Those who only address the fundamental questions of desirability may run the risk to be taken for a technology pessimist. Those ethicists will be accused of fighting a rearguard action. But those who only address the implementation side of new technology may be taken as a so-called 'statutory woman', only functioning as a type of window dressing. They may forget to reflect on the desirability of developments in biomedical practice. By on the one hand addressing more fundamental debates (e.g. chapter 3 and chapter 7 ), and on the other hand developing and proposing conditions for a morally sound clinical practice (e.g. chapter 4), this thesis tries to keep the balance between the Scylla of being superseded and the Charybdis of functioning solely as a lubricant for technological developments. 



\section{Chapter 2}

Dealing with uncertainties:

ethics of prenatal diagnosis and preimplantation genetic diagnosis to prevent mitochondrial disorders

Bredenoord AL, Pennings G, Smeets HJ, de Wert G Human Reproduction Update 2008 Jan-Feb;14(1):83-94 


\section{Abstract}

This chapter aims to address the ethical issues regarding prenatal diagnosis (PND) and preimplantation genetic diagnosis (PGD) of mitochondrial disorders. Owing to the absence of effective treatment, the prevention of the transmission of mitochondrial disorders is considered to be of key importance. The characteristics of mtDNA, such as heteroplasmy and the genetic bottleneck, make it difficult to estimate recurrence risks correctly and to provide an accurate prognosis for many mtDNA mutations. A limited number of mtDNA mutations allow reliable predictions, though results in the 'grey zone' are problematic. Both PND and PGD for mtDNA disorders are complicated by the interpretation of the test results. As a consequence, these applications confront both clinical practice and society at large with several ethical questions and issues for further debate, among which the acceptability of suboptimal genetic testing, the value and research use of embryos, the evaluation of late abortion, the ethics of PGD for disorders with an incomplete penetrance and variable expression, the possible transfer of embryos with residual health risks, the acceptability of risks and drawbacks of genetic reproductive technology in general, and the scope and limits of reproductive autonomy and professional responsibility. 


\subsection{Introduction}

Mitochondrial disorders or diseases due to the defects of oxidative phosphorylation are mostly severe disorders, caused by defects in energy production and affecting at least one in 8500 individuals. Usually the most energy demanding tissues such as the central nervous system, heart and skeletal muscles, liver and kidney are affected. Mitochondrial disorders cause chronic morbidity and can be fatal. They can be discerned in three groups (Thorburn and Dahl, 2001):

1. Diseases with a known pathogenic mutation in a nuclear-encoded gene.

2. Diseases with a respiratory chain enzyme defect in which no pathogenic mutation has yet been identified (i.e. deficiencies with an unknown genetic cause).

3. Diseases with a known pathogenic mutation in an mtDNA-encoded gene.

Disorders from the first group follow a Mendelian pattern of autosomal recessive, dominant or X-linked disease inheritance. Diseases due to mtDNA-defects (group 3), however, have some specific characteristics.

First, mutations are usually heteroplasmic, i.e. there is a mixture of normal and mutant mtDNA, the level of which can differ among tissues. If the mutant load, i.e. the ratio of mutant to normal mtDNA, exceeds a tissue- and individual-specific threshold, clinical features become manifest, although exact genotype-phenotype correlations are usually lacking even within families.

Second, the mtDNA is exclusively maternally inherited. The percentage heteroplasmy of point mutations in the offspring is related to the mutation percentage in the mother (Chinnery et al, 1998), but extreme shifts in mutation percentages are observed due to the genetic bottleneck (White et al, 1999a,b,c; Carelli et al, 2002), which occurs during oocyte development.

These characteristics make it difficult to provide an accurate prognosis for many mtDNA mutations and to estimate recurrence risks correctly. Owing to the absence of effective treatment (Chinnery et al, 2006), the prevention of the transmission of mitochondrial disorders is of key importance (White et al, 1999a; Graff et al, 2000; Chinnery and Turnbull, 2001; Dahl and Thorburn, 2001; Thorburn and Dahl, 2001; Thorburn, 2004; Jacobs et al, 2005a,b; Brown et al, 2006; Schapira, 2006; Spikings et al, 2006). PND for most mtDNA mutations is, however, troublesome. Three criteria have been proposed to select those mutations for which reliable PND can be offered (Poulton and Turnbull, 2000): 
1. There is a close correlation between the level of mutant load and disease severity.

2. There is a uniform distribution of mutant mtDNA in all tissues.

3. There is no change in mutant load with time.

These criteria seem reasonable, since together they determine the predictability of the phenotype, but it is evident that only few mutations meet these requirements. The correlation between the level of mutant load (genotype) and phenotype is low or unknown for many mtDNA diseases. Furthermore, exact threshold values cannot be defined, and interpreting intermediate levels of mutant load is problematic (the socalled 'grey zone') (Poulton and Turnbull, 2000; Poulton and Marchington, 2000; Thorburn and Dahl, 2001; Brown et al, 2006).

Preimplantation genetic diagnosis (PGD) can be an alternative for PND. PGD presupposes IVF with or without ICSI. One or two cells are dissected for genetic analysis. This usually happens at the 8-cell stage, but PGD is sometimes performed on polar bodies. After the genetic analysis, only the unaffected embryos will be transferred (Health Council of the Netherlands, 2003; Sermon et al, 2004). However, the characteristics of mtDNA genetics are complicating PGD as well. As both PND and PGD to prevent mtDNA and mitochondrial disorders with unknown genetic cause raise specific ethical questions, this chapter aims to clarify the central ethical issues involved. Furthermore, we will pay attention to the issues that need further scrutiny and debate now that the field of mitochondrial genetics is rapidly growing.

As specific moral issues are mainly raised by PND or PGD for diseases with an unknown genetic cause (group 2) and mtDNA-caused disease (group 3), and to be more precise about the specific moral questions, another classification is first proposed to guide the ethical debate. This classification is based on the characteristics of the specific mutations.

\subsection{Heteroplasmic mtDNA mutations}

\subsubsection{De novo mutations with a low recurrence risk}

At least one-third of adults with mtDNA disease have a de novo or sporadic mutation. Parts of these are point mutations, but the majority of these patients have a single mtDNA deletion with no or a low recurrence risk (Chinnery, 2002). Observations by Chinnery et al (2004) suggest that clinically unaffected mothers are highly unlikely to have more than one affected child, but affected women have, on average, a $4 \%$ risk of having a clinically affected child. Examples are the three deletion syndromes: progressive external ophthalmoplegia, Kearns Sayre syndrome and Pearson's 
syndrome. Prenatal testing has been performed for mtDNA deletions, mainly for reassurance, but interpretation of the result in case of a mutation found in the chorionic villous sample would be complex and dependent on the nature of the mutation.

\subsubsection{Inherited mutations}

a. Stable mutations, with a predictable outcome.

PND or PGD is generally accepted for the few mutations that, broadly speaking, provide reliable predictions and meet the three criteria proposed by Poulton and Turnbull (2000). The main examples are the mutations $m .8993 T>G$ and $m .8993 T>C$, leading to the neurodegenerative diseases NARP (neurogenic muscle weakness, ataxia, retinis pigmentosa) and Leigh syndrome. Both mutations have a strong genotype-phenotype correlation and show very little tissue-dependent or agedependent variation in mutant load (White et al, 1999b,c; Dahl et al, 2000). When a test result shows mutant load $<60 \%$, the child is very likely to be healthy. When a test result shows mutant load $>90 \%$, the child is very likely to be affected. Results in between these are much less clear, as for these mutations a grey zone exists between 60 and 90\% mutant load (Tatuch et al, 1992; Ciafaloni et al, 1993). PND for both mutations is feasible and has been offered with good results (Harding et al, 1992; White et al, 1999a,b; Leshinsky-Silver et al, 2003; Steffann et al, 2007), although outliers have been reported as well (Enns et al, 2006).

\section{b. Unstable mutations, with an unpredictable outcome.}

Sufficient data show that for some mutations making reliable predictions is very troublesome. They do not meet the Poulton and Turnbull criteria. An example is the m.3243A>G mutation leading to MELAS (mitochondrial myopathy, encephalopathy, lactic acidosis and stroke-like episodes), one of the most common mtDNA disorders (Poulton and Turnbull, 2000). The m.3243A>G mutation is problematic, because there is no clear genotype-phenotype correlation and the mutation load differs among tissues and changes in time. Previous research has shown that there is a rough relation between the severity of the clinical phenotype, the maternal mutation load and the frequency of affected offspring, but this is not sufficiently predictable for individual cases and exceptions do occur (Chinnery, 2002; Chou et al, 2004). In spite of this, and considering the limitations, PND for the m.3243A>G mutation is being offered (Bouchet et al, 2006).

\section{c. Mutations with an unknown outcome.}

For private or family-specific mutations, insufficient information is available to judge if these mutations allow sufficient reliable predictions and meet the Poulton and 
Turnbull criteria. This makes it difficult to identify potentially healthy offspring. Oocyte sampling might be useful as a preliminary step to determine the distribution of the mutation load and the number of mutation-free oocytes. Although it is not sure that the information obtained is representative for the oocyte and possible future embryo, it could be used for preconception counseling (Poulton and Marchington, 2002). An example is the m.9176T>C mutation. PND for this mutation (leading to Leigh syndrome) seems technically reliable, but the prognostic predictions are not straightforward (Jacobs et al, 2005a,b).

\subsubsection{Homoplasmic mutations}

Homoplasmic mutations are present in $100 \%$ of the mtDNA. Homoplasmic mutations are genetic risk factors for the development of for example Leber hereditary optic neuropathy (LHON, hearing loss or cardiomyopathies). Prenatal testing to assess the mutant load is useless, as the mutation load will always be $100 \%$. However, in some cases prenatal testing might nevertheless be considered for other reasons. For example, for LHON, sex selection could be performed to exclude males. Males have a $50 \%$ lifetime risk of blindness compared with that of $10 \%$ for females. Most male and female patients experience visual loss in their late teens or early 20s (Man et al, 2002). The recurrence risks are well established from previous pedigree analyses (Chinnery, 2002).

\subsection{Prenatal diagnosis for mtDNA disorders: ethical issues}

Prenatal diagnosis for Mendelian and chromosomal disorders has been extensively discussed, predominantly because of the link with selective abortion (see e.g. Katz Rothman, 1986; Sutton, 1990; Clarke, 1998a,b; de Wert, 1999; Buchanan et al, 2000; Steinbock, 2002; Wertz et al, 2003). There is, at least in Western countries, a strong consensus that PND can be morally justified. But what about PND for mtDNA disorders? PND for most mtDNA mutations is complicated by the interpretation of the test results. All publications in the field emphasize the difficulties in making reliable predictions. These particularly regard intermediate levels of mutant load, when ambiguous information must be dealt with. The criteria of Poulton and Turnbull (2000) suggest that a prenatal test should have a strong predictive character, i.e. it should be possible to predict the phenotype with a high degree of certainty. At this moment, for most mtDNA mutations the predictive character of the test results is troublesome. Only testing for the heteroplasmic stable inherited mtDNA mutations yields high chances that the test results are able to predict the phenotype, at least when the test result shows mutant load outside the grey zone, thus, in other words, $<60$ or $>90 \%$. 
Whether genetic testing is acceptable, partly depends on the interpretation of two ethical core concepts: non-directive counseling and informed consent. Key publications in the field of mtDNA disease all emphasize the importance of adequate genetic counseling and carefully obtaining informed consent (e.g. Poulton and Marchington, 2000; Thorburn and Dahl, 2001; Bouchet et al, 2006; Brown et al, 2006). However, both concepts are ambiguous and open for discussion. Different interpretations of these concepts will lead to different views regarding the acceptability of PND for mtDNA disease. Every person involved in mtDNA genetics and reproductive decision-making, whether physician, researcher or otherwise, has, explicitly or implicitly, an ethical opinion about these concepts.

\subsection{Non-directive counseling}

Non-directive genetic counseling is the standard in clinical reproductive genetics. Freedom of choice and patient autonomy are essential goals of genetic counseling (Wertz et al, 2003). However, the meaning of (non-) directivity is unclear: when is a genetic counselor inadmissibly directive? Does non-directivity imply the providing of unconditional support, whatever the clients request (de Wert, 1999)? What should be the ethos of genetic counseling (Clarke, 1998a; Wertz et al, 2003)? Furthermore, it has been emphasized that the social context in which individuals make reproductive decisions is relevant, both on the socio-political level and in a private context (Clarke, 1998b).

When health care professionals counsel patients about preventing an mtDNA disorder, they should be aware of the scope and limits of non-directive counseling. Personal opinions about prevention, PND and PGD may influence their counseling and the decision-making process of their clients. This is particularly important when difficult and precarious decisions seem unavoidable, like in the case of the m.3243A>G mutation leading to MELAS.

\subsection{Informed consent}

There is a strong consensus that informed consent is a necessary condition for medical interventions in general and genetic testing in particular. Consent should be based on the understanding of adequate and accurate information (Kuhse, 2002). This process of informed consent should also encompass the pre-test counseling phase (Abramsky, 2003). Indeed, deciding whether or not to have a genetic test at all is one of the most important steps in the decision-making process. This counts even more for mtDNA disorders, as there are many uncertainties to be explained to and understood by the parents. In other words, all intricacies should be assessed right from the start. Nevertheless, the exact prerequisites of informed consent are subject 
to discussion. Is informed consent a sufficient condition, or merely a necessary condition?

At one end of the scale, any treatment or intervention is acceptable when adequate informed consent is obtained. Informed consent is interpreted as a procedure, with guaranteed ethically acceptable outcomes, provided the procedure of informed consent is carried out correctly and adequate (pre-test) counseling has occurred. Applied to the context of testing for mtDNA mutations, it would be sufficient to clarify prior to testing the possible drawbacks of testing. These possible drawbacks include the considerable amount of uncertainty and the possible false-negative and falsepositive results. Thus, even when there is hardly any predictive information, such as for the private point mutations, or when it is known that only ambiguous information is at hand, as for the m.3243A>G mutation, testing would be justified if clients give informed consent. Parents should be enabled to make their own decision, because it mainly affects their lives and not the doctor's. When parents request prenatal diagnosis for the m.3243A>G mutation, one could decide to offer this and leave the decision up to them, after adequate counseling (Chou et al, 2004). Clearly, this interpretation leaves maximal space for reproductive autonomy. This is usually defined as the right to control one's own procreation unless the state has a compelling reason for denying a person that control (Dworkin, 1993; Harris, 1998). The scope and precise substance of 'compelling reasons' is, of course, the subject of discussion. Most will accept this (negative) right to control one's own procreation, but whether this also implies a positive right of assistance to realize those rights and a correlating duty of the physician is less clear (Nys et al, 2002).

At the other end of the scale, it is argued that informed consent is a necessary condition, but not sufficient. This position adds some substantial preconditions. To be a good doctor, the professional standard requires several conditions that have to be met before a treatment can be offered. The criteria proposed by Poulton and Turnbull (2000) can be perceived this way. Authors who commit themselves to these criteria thus acknowledge that mere informed consent is not enough. There are additional criteria; the amount of false-negative and false-positive results should be limited and the test should have a sufficient predictive character. If not, it should not be offered, even when parents insist.

This more restrictive attitude towards the offering of tests can be founded on several theoretical notions. The first background theory regards the concept of futility, the goals of medicine, and more in particular the goals of PND. According to this view, the available data for most point mutations are simply insufficient to construct a 
balanced informed consent. Even more, testing for these mutations might be called medically futile. The main aim of PND is to inform parents about the health of the fetus, enabling them to make informed reproductive choices. If this information is inaccurate, the goals of PND are lost; the test does not provide clarity and reassurance, but uncertainty and distress. From a moral point of view, testing will become increasingly problematic as the test results become more unreliable.

A second base of a more restrictive attitude towards testing may be classic paternalism. Although autonomy is a worthwhile ideal, in reality illness, uncertainty and other circumstances make people dependent and restrict freedom and autonomy (Gaylin and Jennings, 2003). Informed consent as a sufficient condition is therefore, according to this view, unacceptable. Paternalism may be legitimate, because people are in a far from ideal situation, strongly desiring healthy children. This may affect their autonomous decision-making capacity and therefore, it is not acceptable to burden people with such difficult decisions. Furthermore, patients have to be protected against too high a percentage of false-negative and false-positive or insufficient predictive test results.

A middle road to introduce new applications of PND (and PGD) may be to introduce the test only as part of scientific research. When in doubt about the acceptability of a new application, it could be performed in an experimental setting, with very small numbers of patients. This may be an appropriate format when only very limited information is available about specific mutations, such as the private point mutations, but also for the unstable mutations. After evaluation of the experiences of the people involved, a better-founded decision can be made about whether or not to continue. In this situation, the ethics of medical scientific research would provide guidance. Of course, careful criteria of inclusion should be formulated.

\subsection{Terminating pregnancy}

If the prenatal test indicates the presence of mutant mtDNA, termination of pregnancy is an option. Besides the general ethical, psychological and social difficulties of terminating a pregnancy on genetic grounds (Katz Rothman, 1986; Korenromp et al, 1992; Warren, 1998; Tooley, 1999; Gillon, 2001; Wertz et al, 2003), considering selective abortion after prenatal testing for an mtDNA mutation is further complicated by the uncertainty of the data and the possibility of repeating the testing in the third trimester, which might result in a late termination of pregnancy.

\subsubsection{Selective abortion based on uncertain data}

The uncertainty of the test results may cause additional difficulties when considering the termination of pregnancy. None of the publications in the field of the prevention of mtDNA disease pay attention to this. These additional difficulties arise, first, from a 
psychological perspective. Couples who decide to terminate the pregnancy when there is (some) uncertainty about the severity of the defect or the accuracy of the diagnosis are at higher risk for prolonged grieving after the termination. When the genetic defect of the fetus could not be diagnosed with certainty or when the severity of the defect was uncertain, couples' doubts about the rightness of the decision increase. It seems optimal when as few uncertainties as possible about the effects, prognosis and life expectancy of the future child are left (Korenromp et al, 1992; Garret and Margerison, 2003). For most mtDNA mutations, this is exactly the problem, for both the expression and the penetrance of the disease are uncertain and hard to predict.

Furthermore, ethical difficulties may arise when due to suboptimal data an unaffected fetus may be aborted. Whether it matters ethically that the decision to terminate the pregnancy is based on uncertain data, depends on the moral status assigned to a fetus. Broadly, we can discern three standpoints (Bewley, 2003; Tooley, 1998; Gillon, 2001; Pennings and de Wert, 2003). The first position holds that the embryo is a person from conception onwards, with full human rights, deserving full protection (Sacred Congregation for the Doctrine of Faith, 1987). A variant holds that the embryo, even if not yet considered to be a person, still deserves full protection because of its inherent potential to become a person. Clearly, (selective) abortion is, then, unacceptable. The second position, the gradualist or intermediate view, considers the fetus to have some independent moral status, which is increasing throughout pregnancy (Gillon, 2001). By adopting the gradualist view, the general intuition that an abortion becomes more problematic the later it is performed can be explained (MacMahan, 2002). Selective abortion, then, is considered to be justified only when other interests overrule the moral value of the fetus. These interests might be the health of the mother and the prevention of severe harm to the future child. Terminating a possible unaffected fetus due to suboptimal data might complicate the balance. Also the value assigned to reproductive autonomy is important here. Finally, the third position states that the fetus has no independent moral status. Whether it should be treated with respect and care depends on the intentions of the couple, in particular the woman. Those adhering to the third position have no moral problems with selective abortion if this is the parents' decision. Of course, psychological problems may remain.

Prenatal tests to prevent mtDNA disease, at this moment, are far from perfect. Fetuses that could have developed into healthy children may be aborted. Some will accept this as an unavoidable side-effect of fallible technology. Others will consider this a bridge too far. This, however, is not unique for mitochondrial genetics. Other 
examples exist where pregnancies are terminated when there is a chance that the future child would have been healthy; e.g. Duchenne's muscular disease. For some couples, the only type of PND is fetal sexing, with the option of terminating the pregnancy when the fetus is male. This means that in half of the cases a healthy fetus is aborted (Garret and Margerison, 2003). Another example may be prenatal exclusion testing for Huntington's disease. This test is applied when potential carriers wish to exclude transmission of the mutation to their offspring, but at the same time do not want to know their own genetic status. Here also, an unaffected fetus is aborted in $50 \%$ of the cases (while full testing would have avoided this) (de Wert, 2002; Jacopini et al, 2002; Zoeteweij et al, 2002). These practices set a possible precedent for mtDNA mutations.

\subsubsection{Late abortion}

Previously, we made a distinction between mitochondrial disorders caused by a known pathogenic mutation and mitochondrial disorders with an unknown genetic cause, but with a biochemical deficiency. For the latter group, biochemical (enzymebased) tests may be offered. There are, however, limitations to such testing because, again, the reliability is not optimal. This means false-negative results and a lot of 'noise' in the testing results (Bouchet et al, 2006). A normal test result in week 10 cannot exclude an affected child (Faivre et al, 2000). A possible solution would be sequential testing. Some (Faivre et al, 2000; Steffann et al, 2007) suggest performing an additional amniocentesis in week 28-30 of gestation, e.g. to avoid false-negative results due to possible late expression of the biochemical defect. Testing at several times, including the third trimester, may technically be the best option to decrease the uncertainty, but it raises some new ethical issues. First, these tests may cause fetal loss. Second, when a test in week 28 shows an adverse outcome, a late, third trimester, abortion needs to be considered.

In the discussion about late abortion, the legal and moral status of late abortion should be discerned. Whereas in some countries late abortion is prohibited, other jurisdictions do not make a distinction between early and late abortion (e.g. World Health Organisation, 2003; Visser et al, 2005). For the ethical discussion, the moral status of the fetus is a crucial point. For people assigning absolute or high moral status to the embryo right from the start, the timing of abortion is ethically irrelevant. Those assigning no independent moral status to the fetus will leave the decision in the hands of the couple, more specifically the mother. Late abortion is most complex for those who assign a growing moral status to the fetus (the gradualist view). After all, as the fetus develops, its moral value evolves as well (Sprang et al, 1998).

Viability, usually defined as the (potential) ability of the fetus to survive outside the uterus, is often regarded as a highly relevant moral dividing line (Chervenak et al, 
1995; Sprang et al, 1998). It is presumed to exist after 24 weeks of gestation and not prior to 20 weeks. The weeks in between form a grey zone (Human Fertilisation and Embryology Act, 1990; Gans Epner et al, 1998). Others deny or question the ethical importance of viability (Fost et al, 1980; Rhoden, 1986; Grimes, 1998). In any case, late abortion causes an additional grief for both parents and staff (Boxall and Turner, 2003). The further the pregnancy is progressed, the more difficult the decision to terminate becomes for couples (Korenromp et al, 1992). No doubt, developments in preventing mtDNA disease require further reflection regarding the moral weight of viability and the ethics of late abortion.

\subsection{Preimplantation genetic diagnosis for mtDNA disorders: ethical issues}

Although many ethical issues raised by PGD are the same as for PND, PGD is not just a simple alternative to PND. After all, there are preliminary moral issues surrounding assisted reproductive technology in general, and IVF and ICSI in particular. Most authors and practitioners do not support these objections to assisted reproductive technology (e.g. de Wert, 1998a; Kuhse, 2002; Pennings and de Wert, 2003). The objections to PGD mainly regard risk and safety issues, the discarding of embryos, the selection of embryos, the slippery slope and fears for eugenics (King, 1999). Notwithstanding these objections, there is a strong consensus that PGD is justifiable as well, at least for severe genetic disorders (Fasouliotis and Schenker, 1998; Buchanan et al, 2000; Verlinsky and Kuliev, 2000; Steinbock, 2002; Robertson, 2003; Klipstein, 2005; Kuliev and Verlinksy, 2005a,b; MacMahan, 2005; Dresser, 2006). There also seem to be some obvious advantages of PGD compared with PND, such as the possibility to avoid a termination of pregnancy (Fasouliotis and Schenker, 1998; Verlinsky and Kuliev, 2000; Lashwood, 2003). One could say this advantage comes even more to the fore when selective abortion is complicated by the uncertainty of the data.

As far as we can see from the literature, PGD for mtDNA disease has currently been reported only once. It has been successfully applied in a family with the m.8993T>G mutation (Steffann et al, 2006) and seems technically feasible for other mtDNA mutations as well (Brown et al, 2006). However, since the chance of making reliable predictions is higher, the opportunities for the stable inherited heteroplasmic mutations, including the m.8993T>G mutation, seem better than for the other mutations (Steffann et al, 2006). The uncertainty that, at this moment, is inherent in PND of most mtDNA mutations complicates PGD for mtDNA disorders as well. Some ethical topics are similar to those we have discussed in the context of PND, especially the issues of genetic counseling and informed consent. We will not repeat these issues here. But PGD also raises additional ethical questions. Whereas in case 
of PND, informed consent and reproductive autonomy may be restricted on grounds of two background theories, PGD brings in a third background theory, regarding the special responsibility the physician takes in this field. Furthermore, PGD might give greater choice to couples, which also can raise new issues. Before we will discuss the implications of these contextual factors of PGD combined with the uncertainty of mtDNA genetics, the issue of embryo research will be briefly considered.

\subsection{Embryo research}

Preclinical embryo research can be useful for two reasons: first, to develop reliable preimplantation diagnostic tests and, secondly, to study mtDNA inheritance, i.e. for scientific research regarding early embryonic development and the distribution of mtDNA. One publication in the field explicitly refers to this need for embryo research (Steffann et al, 2006). To investigate mtDNA inheritance, the embryos should carry an mtDNA mutation. Embryo research needs debate for several reasons.

First, views regarding the moral status of the embryo are important, both for the evaluation of research using surplus embryos and because it may be necessary, due to the unsuitability of surplus embryos, to create embryos. Although we notice a slow move towards a more liberal regulation, creating embryos for research purposes is at this moment for many countries unacceptable. It is also prohibited by the Convention on Human Rights and Biomedicine (Council of Europe, 1997; Strong, 1997; Olsthoorn-Heim et al, 2006).

A second point is whether asking women to donate oocytes for research is morally justified. Dean et al (2003) note that obtaining oocytes or embryos from women with mtDNA mutations is difficult. One could of course ask a carrier of an mtDNA mutation to create and give up embryos for research, but the question remains whether this is justified. Oocyte donation for research is a topic of international debate, for two reasons. Firstly, what about the proportionality, also in light of the health risks for the donor. And secondly, there is debate regarding how to ensure voluntariness and how to avoid undue pressure (Hyun, 2006; Mertes and Pennings, 2006). To assess the proportionality, two distinctions may be morally relevant. A first distinction can be made between oocyte donors who are subfertile, opting for IVF anyway and oocyte donors who will undergo IVF solely to donate oocytes for research. The development of in vitro maturation is morally relevant here as well, since it implies that hormonal stimulation is no longer necessary. A second distinction can be made between healthy women donating an embryo for research purposes and patients donating, thus, women carrying an mtDNA mutation. In the latter case, the woman may already have a treatment relationship with a particular physician or hospital. This may be both an argument for and against donating. Defenders of oocyte donation for research 
argue that due to this treatment relationship the woman will be particularly motivated to make a contribution. Others fear undue influence and pressure by the physician to donate embryos. Clearly, further debate about embryo research and oocyte donation for research, also in the context of mtDNA genetics, is needed.

\subsection{The responsibility of the health care professional}

Assisted reproductive technology is characterized by the fact that the doctor is involved in the normally two-person enterprise of making a baby (Purdy, 1998). There is a strong consensus that the involvement of the fertility specialist in the conception of the child implies some kind of responsibility towards this child (Health Council of the Netherlands, 2003; Human Fertilisation and Embryology Authority, 2003; Purdy, 1998; de Wert, 1998b, 1999). However, the precise implications of this professional responsibility, its extent and limits, are much less clear, both for the fertility specialist and for the geneticist (Clarke, 1998b). Furthermore, a tension can exist between the responsibility of the health care professional and the reproductive autonomy of the parents. What if parents, carrying an mtDNA mutation, ask for medically assisted reproduction?

Some emphasize the monopoly position and social role of the physician, leading to a prima facie obligation to assist parents asking for help (Harris, 1999). If a procreative choice or technology can lead to suboptimal circumstances for the resulting children, this does not automatically mean that reproductive autonomy should be restricted immediately. Objections have to be strong and convincing (e.g. Robertson, 1994; Burley and Harris, 1999). Although the starting point is to maximize well being, doctors may do less than the best and thus cooperate to suboptimal outcomes when there are 'good reasons' (Savulescu, 1999). Similar tensions between professional responsibility and reproductive autonomy can arise once a physician and the parents agreed about starting an IVF/PGD trajectory (de Wert, 1999; Pennings et al, 2003). It can be argued that the final decision is the responsibility of the woman, because in most cases she is the main caregiver to her (disabled) child (Wertz et al, 2003) and because denying women effective means of controlling their own fertility has harmful consequences for families, women and children (Warren, 1998). Others, however, argue that making these decisions is a joint enterprise, and that the professional may still be entitled to refuse assistance, taking into account the welfare of the child (de Wert, 1999). Both the ESHRE PGD Consortium (2005) and the Human Fertilisation and Embryology Act (1990) require the welfare of the child to be considered in the decision to offer assisted reproductive technologies. But how to make it operational in everyday practice? 


\subsection{The welfare of the child}

There is an ongoing debate about the practical application of 'the welfare of the child' (Blyth and Cameron, 1998; Brewaeys, 1998; Mumford et al, 1998). Three different evaluation standards are used to assess whether offering assisted reproductive technology is acceptable [see Table 1] (Pennings, 1999; de Wert, 1999). Here, we will translate them to the question whether offering IVF/PGD is acceptable, based on the quality of life, or the expected health status, of the resulting child.

The first is the 'maximum welfare principle': one should not knowingly and intentionally bring a child into the world in sub-ideal circumstances. Based upon this principle, the overwhelming majority of the candidate parents would be excluded. All possible deviations from the ideal life are criteria of exclusion. According to this principle, most if not all mtDNA mutations should probably be excluded for PGD, as, first, in the majority of cases, a healthy child cannot be guaranteed and, secondly, due to the maternal inheritance, the mother may develop the disease (except for mutations de novo). A sick mother deviates from ideal circumstances.

The second standard to assess the welfare of the child is the 'minimum threshold principle', or, more particularly, the 'wrongful life' or 'worse than death' criterion: the only reason not to bring a child in the world is when the child would have been better off not to live at all. This principle entails a minimal conception of the responsibility of the health care professional and leaves almost absolute space for reproductive autonomy. According to this principle, offering IVF/PGD is acceptable for all mtDNA mutations as long as the test result is predictable. In case the test result may be suboptimal, the expected life standard of the resulting child may not be worse than death. Thus, when living with LHON, in the worse case scenario the child would become blind at young age. Although a serious impairment, most would agree that this still remains a life worth living. Physicians should therefore, based on this standard, offer IVF/PGD. Whether other mtDNA mutations will enable the child to live a life worth living is debatable and also depends on the severity of the disease. In particular for mutations with a highly variable expression, such as the m.3243A>G mutation leading to MELAS, making judgments is complicated.

Finally, the third, intermediate, principle is the 'reasonable welfare principle': assistance of the health care professional is justified when there is reasonable chance of having a reasonably happy child (or acceptable life standard). A contraindication for assisted reproduction would be a 'high risk of serious harm' (de Wert, 1999; Benatar, 2006). Whether a child with one of the mtDNA mutations will have an 
acceptable life standard depends on the harm-probability ratio of the specific mutation.

Table 1 Standards to assess the welfare of the child

Maximum welfare principle
IVF/PGD is only acceptable in ideal circumstances
Minimum threshold principle
IVF/PGD is acceptable when the expected life standard of the resulting child will not be worse than
death
Reasonable welfare principle
IVF/PGD is acceptable when the expected life standard of the resulting child is reasonable and there
is no 'high risk of serious harm'

Thus, if one adheres to the maximum welfare principle, PGD to prevent any mtDNA disease would be unjustified, since, currently both a healthy child and the long-term availability of a healthy mother cannot be guaranteed. Both other criteria, however, leave ample room for discussion.

\subsection{Responsible parenthood and parental responsibility}

Obviously, discussions about responsible parenthood are not exclusive for mtDNA disease. This discussion regards all genetic diseases as a result of which one of the parents has a fair chance to become seriously ill (ESHRE PGD Consortium, 2005). All mtDNA disorders follow a maternal mode of inheritance. A female carrier may have no or mild symptoms (especially if she has a lower mutant load), but she may also have a substantial mutant load and develop severe symptoms. Her life expectancy may be seriously reduced. The physician might consider not offering assisted reproduction, because his or her professional responsibility requires that he or she should not burden a child with a possibly sick mother. Furthermore, the child might lose its mother at a young age. Counter-arguments are that one-parent families are often doing well. In other cases, we cannot predict the future health of the parents either. Furthermore, according to both the minimum threshold principle and the reasonable welfare principle, living with one parent or a sick mother is acceptable. Last but not least, for most mtDNA mutations, it is debatable whether the health of the mother is poor enough to justify a limitation of their reproductive autonomy. If the mother is affected, much depends on the bearing power of the prospective father. 


\subsection{The transfer of embryos with residual risk}

A successful IVF/PGD procedure normally provides the availability of several embryos. In that case, the couple can make a decision about which embryo to transfer, whereas with PND the only choice is whether to terminate the pregnancy (Draper and Chadwick, 1999). The most straightforward scenario would be if one or two embryos without detectable mutant load are available. In this case, we can assume these embryos will be transferred. However, in many cases, PGD of mtDNA disorders does not enable people to eliminate genetic risk, but only to reduce it, both for heteroplasmic and homoplasmic mutations.

Characteristic for mtDNA heteroplasmic mutations is that embryos and people often have a certain amount of mutant load, which can vary from 0 to almost $100 \%$. Most authors regard only embryos with undetectable or very low amounts of mutant mtDNA as suitable for transfer (Thorburn and Dahl, 2001; Dean et al, 2003; Jacobs et al, 2005a,b). However, when performing PGD for, e.g. the m.3243A>G mutation, it is not inconceivable that only embryos with residual health risks may be available. Would it be morally justified, then, to transfer an embryo with some mutant load? And what should the cut-off point be? Clearly, the lower the cut-off point, the more embryos are discarded and, thus, the lower the take home baby rate - and vice versa. Another issue is whether and when the clinicians' responsibility to transfer the best possible embryo would imply to start another IVF/PGD cycle in order to obtain mutant-free embryos. Savulescu (2001) defends the so-called 'procreative beneficence' principle, arguing that couples should select the embryo which is expected to have the best life. They should use all the available information and choose the option that is the most likely to bring about the best outcome. This view raises the question how many IVF/PGD-cycles could (or should) justifiably be done before one chooses to implant an embryo with a certain mutant load.

The application of PGD for homoplasmic mutations, such as LHON, goes one step further as all embryos will carry residual health risks. Prenatal testing to assess the mutant load is useless, as the mutation load will always be $100 \%$. PGD could, however, be considered for sex-selection to exclude males. These males have a $50 \%$ lifetime risk of blindness compared to that of $10 \%$ for females. Why not transfer a female embryo, if the prospective parents insist and if a healthy embryo is out of the question? Should the physician insist on only replacing female embryos? No doubt, this application is controversial. In these cases, a conflict may arise between the prospective parents' reproductive autonomy and the responsibility of the reproductive physician to take into account the welfare of the future child. Furthermore, the slippery slope argument should be considered, as applications like 
this may open the door for sexing for all disorders in which the mutation's penetrance and/or the expression is lower or milder in one sex (Pennings, 2002).

In any case, PGD for mtDNA disease brings the classic application of PGD up for discussion. 'It is one thing, it could be argued, for couples to refuse to undertake P[I]GD, but quite another for them to request that an affected embryo be implanted' (Draper and Chadwich, 1999). Intuitively one could argue that the primary goal of PGD is quite the opposite: the birth of healthy children. However, sometimes this goal seems out of reach. Though PGD may not be ideal in these situations, some couples and clinicians nevertheless may consider PGD to be the best (or the least unfavourable) option.

\subsection{Polar body analysis}

Polar body analysis can potentially be used in the diagnosis of mtDNA disease (Briggs et al, 2000; Dean et al, 2003). The first polar body arises just before ovulation and can be used to determine the genetic status of the oocyte. To improve the reliability of the genetic analysis, it is possible to test the second polar body as well. This appears after the sperm cell has penetrated the oocyte. Limitations of polar body analysis are generally twofold. First, it only reveals chromosomal aberrations that already have taken place. New chromosomal deficiencies will not be detected. Second, with regard to Mendelian disorders, only the genetic material of the mother is analysed (Verlinsky and Kuliev, 2000; German National Ethics Council, 2003, Kuliev and Verlinsky, 2005b; Soini et al, 2006). These limitations, however, do not apply to the polar body analysis for mtDNA mutations, because only the maternal mitochondrial contribution needs to be determined. However, at the moment blastomere biopsy seems to have a higher diagnostic efficiency (Briggs et al, 2000; Dean et al, 2003).

To those who oppose embryo testing and selection, analysis of the 'first' polar body has an ethical advantage; after all it entails oocyte selection (Dean et al, 2003). In countries where PGD is prohibited, e.g. in Germany, polar body analysis is considered to be an alternative. Polar body analysis is, however, performed internationally on a very small scale, mostly combined with blastomere biopsy (German National Ethics Council, 2005). When carried out in combination with either second polar body or blastomere biopsy, the reliability may be higher, but the initial goal, by-passing embryo selection, is not met.

\subsection{Genetic testing of minors}

Some authors genetically test children born after PND or PGD. Some have measured postnatally the mutant load in the cord blood (White et al, 1999a; Graff et 
al, 2000). Others do not assess the presence of the mtDNA mutation 'for ethical reasons' (Jacobs et al, 2005a,b; Bouchet et al, 2006). The reasons for or against testing are, however, not made explicit by these authors, although it seems that testing is performed to confirm the absence of mutant mtDNA (Graff et al, 2000).

As genetic testing of minors is a controversial issue, it is important to carefully distinguish the reasons for and implications of testing. In this case, testing a (thus far) healthy child after birth could probably be done for two reasons. The first reason would be to remove the residual uncertainty and to confirm the test results. This will probably be the case when a 'healthy' child is expected. When the child, as expected, does not have mutant mtDNA, then the parents are reassured. However, when the mutation is present against all expectations, a neutral affirmation may turn out to be a tricky genetic prediction. Predictive genetic testing of minors is an ethically sensitive topic. There is a strong consensus that such testing is only acceptable when there are clear health advantages for the child. The situation is ethically less clear when the medical benefit is uncertain or absent, when there are no preventive or other therapeutic measures available, or when the disease will manifest at adult age. Arguments against predictive genetic testing of these minors regard the autonomy of the child, its privacy and the child's right not to know (Clarke, 1998b; de Wert, 1999; Borry et al, 2006). For mtDNA disease, there are currently no real treatment options (Chinnery et al, 2006). The medical benefit of testing is therefore unclear and an alternative could be just to wait and see, as there are insufficient empirical data regarding the psychosocial consequences of predictive testing in minors (Evers-Kiebooms, 2006).

A second reason for testing may be the scientific surplus value: to increase our knowledge about mtDNA inheritance and the genotype-phenotype relation. However, none of the publications in the field mention this reason. In the case of a test performed for scientific reasons, the ethics of non-therapeutic medical research with incompetent subjects is the leading framework (World Medical Association, 1964/2008; Council for International Organizations of Medical Sciences, 2002). In our opinion, it should be made clear whether testing children born after PGD and PND for mtDNA disease is for medical purposes or for scientific purposes, since this would imply a different ethical framework. Owing to the sensitivity of this subject, further debate is needed about the pros and cons of genetically testing children born after PND or PGD for an mtDNA mutation.

\subsection{Mitochondrial DNA disease a new indication for PGD?}

PGD was introduced as a technique to prevent monogenetic disorders that result in serious harm. Indications for PGD were similar to those accepted for PND. In recent 
literature, attention has been paid to new applications of PGD, beyond the strict criteria for PND (Kuliev and Verlinsky, 2005a,b). Whether a wider scope of application is acceptable is open for debate (Pennings and de Wert, 2003; Robertson, 2003; UNESCO, 2003; Shahine and Caughey, 2005; de Wert, 2005; Health Council of the Netherlands, 2006; Niermeijer et al, 2006). It may be useful for the debate about the ethics of PGD for mtDNA disease to make a comparison with analogous cases, such as PGD for cancer susceptibility genes. PGD for adult-onset cancer predisposition genes, such as the BRCA mutations and HNPCC (hereditary non-polyposis colorectal cancer), is controversial for several reasons. First, due to the incomplete penetrance of most of the relevant mutations, some carriers will not be affected. Second, due to the later age of onset, people can often live for more than 30 years without developing the disease - but there are exceptions, such as LiFraumeni syndrome, causing a high risk of childhood cancers (Rice, 2006). Third, for some disorders prophylactic or surveillance measures are possible, like a double mastectomy (Harris et al, 2005; Kuliev and Verlinsky, 2005a,b; Rice, 2006). Likewise, people with mutant mtDNA may have the chance of never or only later in life develop symptoms. However, they may become seriously ill as well, or even die at young age. Furthermore, effective therapy is currently not available. An important difference between the two types of disorders, however, is that PGD for mtDNA disease cannot always exclude residual health risks, whereas PGD for genes predisposing to cancer can guarantee the birth of children free from the particular hereditary cancer.

To people assigning a certain or high moral status to embryos, PGD is only ethically acceptable when done for good reasons, such as preventing offspring with serious genetic disease. When discussing new applications of PGD, the question therefore is whether the reasons are good enough to select and discard embryos (Robertson, 2003). Some fear a slippery slope. So far, PGD was mainly used for severe single gene disorders. In the near future, it may be increasingly used to select against mild diseases. Arguments in favour of extending the indications for PGD are, among others, reproductive autonomy and the prevention of harm. To substantially decrease the risk of cancer by means of PGD constitutes good preventive medicine (Braude, 2006). Performing PGD to prevent mtDNA disorders can also be perceived in this way, although the difficulty to predict the severity of the disease is a complicating factor. Countries have different views about extending the criteria for PGD. In 2006, the HFEA has included certain cancer susceptibility genes as legitimate reasons for PGD (Braude, 2006). PGD for mtDNA disease might therefore be accepted as well. In the Netherlands, the political discussion about extending the criteria for PGD is still ongoing (Handelingen Tweede Kamer, 2005-2006; Health Council of the 
Netherlands, 2006). Meanwhile, health care professionals have different views (UNESCO, 2003).

\subsection{Considering alternatives: genetic relatedness, harm to the child and proportionality}

Thus far we have focused on PND and PGD. However, some alternatives exist to prevent mtDNA disorders. Although (pro)nuclear transfer may become an option in the future (Rubenstein et al, 1995; Roberts, 1999; de Wert, 1999; Thorburn and Dahl, 2001; Jacobs et al, 2005a,b; Spikings et al, 2006; Brown et al, 2006), currently available alternatives include oocyte donation and adoption. A practical problem is the scarcity of donor oocytes. Furthermore, for some, oocyte donation raises the same ethical questions as assisted conception in general, such as the meaning of family values, sexuality, parenthood, gender relations and commodification (e.g. Murray, 1996). Adoption would also be a solution for mtDNA disorders. However, these options result in unrelated or only partially related offspring. These strategies are, therefore, for a lot of couples less desirable. Some authors briefly mention these alternatives (Poulton and Marchington, 2000, 2002; Poulton and Turnbull, 2000; Thorburn, 2004) but they do not, or only briefly address the psychological and moral issues involved (Thorburn and Dahl, 2001; Jacobs et al, 2005).

The main moral question is whether genetic relatedness is that important that the use of costly, suboptimal and time-consuming techniques to prevent an mtDNA disease is justifiable when relatively 'simple' alternatives are available. Some would say that genetic relatedness, among others, is not the true determining factor for the child's well-being (Golombok, 1998). Others mention social pressure, both to become parents, and to have children of one's own (Strong, 1997). Furthermore, if we allow suboptimal outcomes to ensure genetic relatedness, then we have generated a strong argument in favour of reproductive cloning (Savulescu, 1999). After all, cloning ensures the highest degree of genetic relatedness. The importance of genetic relatedness is often assumed, but further reflection about the value and significance of the desire for a genetically related child is required (Graumann and Haker, 1998; Sparrow, 2006).

An intensive, costly and time consuming treatment for couples at risk can only meet the moral requirement of proportionality, also in light of scarce resources in health care and urgent global questions, when there is some degree of certainty about which embryo to transfer. The category of stable inherited heteroplasmic mutations (including the m.8993T>G mutation) is in this perspective different from the other categories. However, making proportionality operational in everyday practice is a 
complex issue. It may be helpful to distinguish between people needing IVF anyway because of infertility, and fertile people with a known risk of genetic disease, who want to avoid PND and possibly abortion by having IVF and PGD. The latter couples make a claim to IVF not because they are infertile, but because this is a prerequisite to have PGD. With the development of PGD, new kinds of uses of IVF have arisen (Holm, 1998; Soini et al, 2006). IVF, which started as a fertility technique in the 1980s, has turned into a wheelbarrow for genetic services.

Women carrying a higher mutant load have a stronger likelihood of having a fetus with an intermediate or high mutant mtDNA. Some authors therefore suggest that PGD might, because of the hormonal stimulation, financial costs etc, be more suitable for woman with high mutant mtDNA (Dean et al, 2003; Poulton and Marchington, 2002; Jacobs et al, 2005a,b). Moreover, in case of a mutation with a low recurrence risk (or de novo), PND may be a better option than PGD. After all, in these cases starting an IVF/PGD treatment may be disproportionate. Moreover, the chances to become pregnant are higher with natural reproduction and the risk of an affected child (and subsequently of considering a possible termination) are small.

\subsection{Concluding remarks}

This paper aims to address the ethical issues regarding PND and PGD of mtDNA disorders and mitochondrial disorders with an unknown genetic cause. Extended ethical analysis and interdisciplinary debate will contribute to further guidance and to the adequate management of the challenging questions surrounding the prevention of the different mtDNA mutations. A limited number of mtDNA mutations, in particular the stable inherited mutations, allow reliable predictions, though results in the 'grey zone' complicate testing. Flaws in genetic and biochemical diagnosis of the other mtDNA mutations and the uncertainty that is currently inherent to this field, lead to complex ethical questions. From the unassailable fact that genetic testing in this field is far from perfect we cannot, however, jump directly to the conclusion that all applications are unsound. No doubt, an extended ethical debate and analysis is of utmost importance for the further development of good clinical practice. 


\section{Chapter 3}

PGD to reduce reproductive risk: the case of mitochondrial DNA disorders

Bredenoord AL, Dondorp WJ, Pennings G, De Die-Smulders CEM, de Wert G Human Reproduction 2008 Nov;23(11):2392-401 


\section{Abstract}

This chapter discusses the pros and cons of introducing preimplantation genetic diagnosis (PGD) for mitochondrial DNA (mtDNA) disorders such as NARP (Neurogenic muscle weakness, Ataxia, Retinis Pigmentosa)/Leigh, MELAS (Mitochondrial myopathy, Encephalopathy, Lactic acidosis, and Stroke-like episodes), private mtDNA mutations and LHON (Leber Hereditary Optic Neuropathy). Although there is little experience with PGD for mtDNA disorders, it is reasonable to assume that in many cases, the best one can achieve is the selection of the 'least' affected embryos for transfer. So instead of 'promising' parents a healthy child, PGD in these cases can only aim at reducing reproductive risk. From an ethical point of view, this raises challenging questions about parental and medical responsibilities. The main argument in favour of PGD is that it offers couples at risk the opportunity of reducing their chances of having a severely affected child. Potential objections are manifold, but we conclude that none of them supplies convincing moral arguments to regard risk-reducing PGD as unacceptable. Nevertheless, introducing this new application of PGD in clinical practice will raise further complex issues of determining conditions for its responsible use. 


\subsection{Introduction}

With the expanding unravelling of the causes of mitochondrial disorders, more women at a risk of having a child with a disorder caused by a mitochondrial DNA (mtDNA) mutation are identified. In order to prevent the birth of an(other) affected child, these 'carrier' women ask for prenatal diagnosis (PND). However, as the complexity of mitochondrial genetics negatively affects predictive value, PND is (at least for most mtDNA mutations) not a very satisfactory method of genetic testing (Bredenoord et al, 2008a). In view of this, preimplantation genetic diagnosis (PGD) after IVF is proposed as an alternative. So far, PGD for mtDNA disorders has been performed twice and it has been seriously considered as an option in other cases (Bickerstaff et al, 2001; Steffann et al, 2006). Although PGD is subject to similar limitations as PND, PGD would allow testing prior to pregnancy. Because of this, difficult decisions are avoided about whether or not an unfavourable prediction would be a reason for abortion. Moreover, for those couples who already depend on IVF for fertility problems, PGD would be the logical approach to test for genetic disease.

Nevertheless, PGD for mtDNA disorders is a new and controversial use of the technology. Traditional applications are targeted at Mendelian and chromosomal disorders. In those cases, it is (in principle) certain whether the relevant gene defect is present and which of the available embryos can safely be transferred. When PGD is performed for mtDNA disorders, this will probably be less clear. Although there is little experience with PGD for mtDNA disorders, it is conceivable that in many cases the best one can achieve is the selection of the 'least' affected embryos for transfer. Instead of 'promising' parents a healthy child, PGD in those cases can only aim at reducing reproductive risk. From an ethical point of view, this raises challenging questions about parental and medical responsibilities. Is it acceptable to start an IVF/PGD procedure knowing that there may only be affected embryos? is it acceptable to knowingly bring a child into the world whose health may be (significantly) impaired?

In this chapter, we aim to contribute to the ethical discussion of the developing practice of PGD for mtDNA disorders. We will focus on the question of whether PGD for mtDNA disorders is acceptable. In other words, is PGD acceptable as a means of reducing rather than eliminating genetic risk?

\subsection{Background}

Mitochondrial disorders are usually severe. They involve defects in energy production and affect the most energy demanding tissues such as the central nervous system, heart and skeletal muscles, liver and kidneys. The clinical phenotype of mitochondrial diseases is extremely variable, affecting patients at any age and in a wide variety of 
tissues. Mitochondrial diseases cause chronic morbidity and can be fatal at young age. In view of the absence of effective treatment (Taylor and Turnbull, 2005; Chinnery et al, 2006), preventing the transmission of mitochondrial disorders is considered to be of key importance (White et al, 1999a; Graff et al, 2000; Chinnery and Turnbull, 2001; Dahl and Thorburn, 2001; Thorburn and Dahl, 2001; Thorburn, 2004; Jacobs et al, 2005; Brown et al, 2006; Schapira, 2006; Spikings et al, 2006).

Mitochondrial disorders caused by a pathogenic mutation encoded in the nuclear DNA follow a Mendelian pattern of inheritance, causing (in cases where the molecular defect is known) no specific problems for prenatal or preimplantation genetic testing. However, many mitochondrial diseases are caused by a pathogenic mutation in an mtDNA-encoded gene. Some of these mutations are homoplasmic, meaning that only mutant mtDNA is present in all tissues of an affected individual. Genetic testing aimed at selective abortion or selective transfer is generally not a useful option in homoplasmic mtDNA mutations (we will shortly discuss a possible exception to this). Homoplasmy will be present in all embryos and fetuses of a woman carrying the mutation, since mtDNA is always maternally inherited.

Most mtDNA mutations, however, are heteroplasmic. This means that there is a mixture of normal and mutant mtDNA, the level of which can differ among tissues. If the mutant load, i.e. the ratio of mutant to normal mtDNA, exceeds a tissue- and individual-specific threshold, clinical features become manifest. However, exact genotype-phenotype correlations are usually lacking even within families. With regard to heteroplasmic mtDNA mutations, genetic testing aimed at selective abortion or transfer may be an option. In this chapter, we will discuss the possible role of PGD, even though many considerations will 'mutatis mutandis' also apply to PND.

Preventing the transmission of mtDNA disease is fraught with uncertainties. In view of this, the mitochondrial research community has developed criteria for ascertaining phenotype predictability. These criteria were developed in the context of PND (Poulton and Turnbull, 2000). When we would extrapolate these criteria to PGD, PGD could reliably be offered when there is:

1. a close correlation between the mutation load and disease severity;

2. a uniform distribution of mutant mtDNA in all blastomeres;

3. no change in mutant load with time.

Only few mutations fulfill these criteria and even in those cases exceptions may occur. Owing to the existence of many different types of mtDNA mutations, we earlier proposed a further classification (Bredenoord et al, 2008a). We will use this classification to illustrate the moral difficulties and opportunities of PGD. 
The first group regards de novo mutations, but PGD will not often be considered an option for this category as the recurrence risks are fairly low (Chinnery et al, 2004).

The second group regards stable mutations, with a predictable outcome. The main examples are the mutations m.8993T>G and m.8993T>C, leading to the neurodegenerative diseases NARP (Neurogenic muscle weakness, Ataxia, Retinis Pigmentosa) and Leigh syndrome. Both mutations have a strong genotypephenotype correlation and show very little tissue-dependent or age-dependent variation in mutant load (White et al, 1999b,c; Dahl et al, 2000). PGD has been reported once for the m.8993T>G mutation, which resulted in the transfer of two mutant-free embryos, leading to the birth of one thus far healthy child (Steffann et al, 2006). For these two mutations, reliable predictions can only be made if testing reveals a mutant load below $60 \%$, meaning that the child is very likely to be healthy, or above $90 \%$, in which case the child is very likely to be affected. There is a grey zone between 60 and 90\% mutant load (Tatuch et al, 1992; Ciafaloni et al, 1993). This means that also with regard to the stable mtDNA mutations that fulfill the criteria mentioned above, PGD may lead to difficult questions. What if only embryos with a mutant load in the grey zone turn out to be available? Would it be acceptable to transfer these if that is what the couple requests? Is PGD acceptable if it may lead to such dilemmas? Or should its use be restricted to cases where grey zone outcomes are not to be expected?

The third group regards unstable mutations, with an unpredictable outcome. An example of this is the m.3243A>G mutation leading to MELAS (mitochondrial myopathy, encephalopathy, lactic acidosis and stroke-like episodes), one of the most common mtDNA disorders (Poulton and Turnbull, 2000). The mutation shows a capricious development and genotype-phenotype correlations do not show a consistent pattern (Chinnery, 2002). However, according to experts in the field, a fairly low mutant load generally gives a better prognosis than a fairly high one. With respect to PGD, this implies that transfer of embryos with a low mutant load can be expected to lead to a higher chance of unaffected or mildly affected children. However, even in the more favourable cases (when embryos with a low mutant load are available) the child may still be affected.

The fourth group contains mutations with an unknown outcome: the private point mutations, which are patient or family-specific mutations. Characteristic for these mutations is that very little is known about them. Insufficient evidence exists to decide whether they follow the criteria above and thus allow reliable predictions. As with the m.3243A>G mutation leading to MELAS, PGD may nevertheless be an option: the transfer of an embryo with a low mutant load can be expected to lead to a higher chance of an unaffected or mildly affected child, but also here exceptions may occur. 
As a final illustration of the difficulties surrounding PGD for mtDNA disorders, we point at the fifth group of homoplasmic mutations. Homoplasmic mutations are present in $100 \%$ of the mtDNA. The main example of this group is LHON (Leber Hereditary Optic Neuropathy). Most patients, usually males, experience visual loss in their late teens or early 20s. About $50 \%$ of men and $10 \%$ of women carrying a pathogenic mtDNA mutation actually develop Leber's disease (Huoponen et al, 2002; Man et al, 2002; Hudson et al, 2007). PGD for LHON with selective transfer of female embryos has been reported once, but this did not result in a pregnancy (Bickerstaff et al, 2001). As the penetrance of the disease is considerably higher in men than in women, PGD followed by sex selective transfer of female embryos would lower the risk of blindness in comparison with natural reproduction from 30 to $10 \%$ (Spruijt, 2007). This $10 \%$ risk is unavoidable. Here again, the issue is whether PGD is acceptable if, instead of eliminating the risks of disease transmission, it will at best lead to risk reduction.

\subsection{Arguments in favour of PGD}

At least three arguments can be advanced for offering couples PGD for mtDNA mutations. Notwithstanding the limitations of genetic testing for mtDNA mutations, both PND and PGD offer couples at risk the opportunity to reduce their risk of having a severely affected child. It contributes to reproductive confidence. PGD not only has the additional advantage of testing prior to pregnancy, but also of testing several embryos in one cycle. This enlarges the chances of obtaining embryos without the relevant mutation or with a relatively low mutant load (or in case of PGD for LHON: female embryos). Ideally, one would only transfer embryos without a (detectable) mutant load. But in cases where these are not available, PGD for heteroplasmic disorders such as NARP/Leigh, MELAS and the private point mutations at least allows transfer of the embryo with the lowest mutant load. In such cases, selective transfer of the embryo with the lowest mutant load may still be beneficial with respect to (i) the risk of developing the disease and (ii) the severity of the disease. With regard to LHON, selecting the least affected embryo is no option, as all embryos will have a $100 \%$ mutant load. However, since the penetrance of the disease is sexdependent, selecting female embryos will reduce the risk of clinical disease in comparison with spontaneous conception from 30 to $10 \%$. The main argument for PGD would thus be that it allows couples at risk to avoid the higher risks of natural reproduction, by eliminating embryos with a very high mutant load.

This is indeed a limited advantage. For couples at risk who definitely want an unaffected child, PGD will not be an attractive option, except when mutation-free embryos are available. However, due to the limited experience with PGD for mtDNA 
disorders, it is not clear how often that will be the case. Studies done by Steffann et al (2006) show that, for the m.8993T>G mutation leading to NARP, mutant load among embryos is likely to be skewed. This means that low mutant load or mutationfree embryos may well be available. However, no such information is available for other mtDNA mutations. Other couples may be inclined to accept transmitting certain health risks if - short of refraining from having a genetically related child - that is their only alternative. For those couples, PGD would be of value by allowing them at least to limit their reproductive risks. Furthermore, adding PGD seems a logical step for those already having an indication for IVF due to fertility problems.

The second argument in favour of PGD concerns reproductive autonomy. This is usually defined as the right to control one's own procreation unless there is a compelling reason for denying a person that control (Dworkin, 1993). The rationale behind this principle is that in a liberal democratic society, the presumption is that people should be free to make their own choices according to their own values (Harris, 2007). Denying couples access to PGD for mtDNA disorders is only acceptable when good and sufficient reasons exist to do so. We will discuss below whether indeed good and sufficient arguments exist for not offering PGD.

Thirdly, as 'the proof of the pudding is in the eating', doing PGD will generate important new scientific data to guide counseling and decision-making in future cases. Analysis of embryos not selected for transfer, and follow-up studies of the children born after PGD, can be expected to considerably contribute to our knowledge.

\subsection{Arguments against PGD: valid objections?}

What are the possible objections to PGD for mtDNA disorders-and are these objections valid? Do they undermine the acceptability of PGD for mtDNA disorders?

\subsubsection{PGD for mtDNA disorders does not respect the welfare of the child}

In a recent report by the Health Council of the Netherlands, PGD is defined in terms of allowing couples at high genetic risk to have a child not affected by the relevant disease (Health Council of the Netherlands, 2006). Similarly, in its 'Guidance on Preimplantation Testing', the British Human Fertilisation and Embryology Authority (HFEA) states that the purpose of PGD is to provide information that allows unaffected embryos to be selected for transfer' (HFEA, 2003). Both reports define the goal of PGD as providing couples at risk to have a child not affected by the particular disease. This must of course be qualified: unaffected children can never be guaranteed with certainty, also because a small chance of misdiagnosis is an inherent part of PGD (and this was even more so in the early days of PGD; before the introduction of linked markers, there was a $4-8 \%$ chance of misdiagnosis). But, 
as shown by the examples given above, PGD for mtDNA mutations is a special case, in that it is not the technical limitations that cause the residual risks, but an embryo at (small) risk is knowingly transferred. Transferring unaffected embryos cannot, even with qualifications, be seen as the aim of the procedure.

A first possible objection to PGD for mtDNA disorders may therefore be that it is at odds with the welfare of the future child. It is widely acknowledged that in reproductive medicine, the welfare of the child should be an important consideration. In this field, doctors have a dual responsibility: towards the couple asking for help and towards the child conceived by his/her assistance. With regard to PGD for mtDNA disorders, the question arises whether it is acceptable to knowingly conceive a child with a (significantly) higher risk of developing a disease. This is first of all a question that the future parents should ask themselves. However, the active part played by doctors in the conception of the child means that they cannot comfortably hide behind parental autonomy. After all, when offering IVF/PGD to carriers of mtDNA mutations, doctors are actively involved in bringing a child into the world whose health may be seriously compromised. Are not doctors in those cases inducing rather than reducing health risks? If so, whether this would be morally acceptable is strongly connected to one's view regarding the responsibility of the physician towards the welfare of the child.

Earlier, we described three possible accounts of what the appeal to the welfare of the child would entail (de Wert, 1999; Pennings, 1999; Bredenoord et al, 2008a). These standards are developed to assess whether reproduction and as a consequence offering medical assistance to reproduction would be justifiable. According to the 'minimum threshold standard', offering assisted reproduction technology is acceptable as long as the future child can be expected to have a life that reasonable persons would not regard as 'worse than death' (Harris, 2000; Cavaghan, 2007). Applied to our discussion, the standard entails that transferring embryos at risk is acceptable, provided the clinical manifestations of the disease are not so severe that an affected child would be better off dead. With regard to sex selection in PGD for LHON, the worse case scenario is that the girl would become blind at a young age. Although a serious impairment, most would agree that this still remains a life worth living. Other things being equal, the welfare of the child does not provide a valid argument against doctors offering IVF/PGD. Also the other mtDNA mutations will probably enable the child to live a life worth living, though this may depend on the severity of the clinical symptoms. In particular, for mutations with a highly variable expression, such as the m.3243A>G mutation leading to MELAS, judgements will be complicated. The reasoning behind this first standard is that even if the child can be 
expected to have a life burdened by disability or disease, bringing it into existence would normally not amount to harming it. This is because 'this' child could not possibly have had a better life (Parker, 2007). The only real alternative would have been non-existence (Parfit, 1984). According to the proponents of this standard, the child can only be harmed by a life so awful that it would be worse than not living at all. Insofar as violating the interests of the future child is concerned, this line of reasoning is generally accepted. There is, however, less consensus about whether this settles the issue with regard to justifying assisted reproduction. Many argue that even if being brought into the world does not harm the child, this does not make it a matter of moral indifference what quality-of-life the child can be expected to have (Arras, 1990; Steinbock and McClamrock, 1994; Parker, 2005). Although no harm is done in the sense of a violation of the child's interests, it is still meaningful to say in a more general sense that bringing a (seriously) handicapped or diseased child in the world is to cause avoidable harm. Since doing so may fall short both of parental and professional responsibilities, the minimum threshold standard strikes us as 'aiming too low' (Glover, 2006).

At the other end of the spectrum, we find the 'standard of maximum welfare'. It says that reproduction, and as a consequence medical assistance to reproduction, is only acceptable if it leads to a child with the best possible quality-of-life. This would urge us to reject all forms of PGD for mtDNA disorders. Even in cases where unaffected embryos would be available for transfer, the (risk of) disease of the mother would remain a liability for the child's quality-of-life. However, this standard is also problematic. The difficulty here is that it would rule out far too much. If we were to take maximum welfare seriously as a criterion, should IVF still be available for couples living busy lives with both partners having demanding jobs? Or for couples of advanced age or of modest financial means? As the circumstances are always less than ideal, maximum welfare effectively denies reproductive assistance to most if not all. Moreover, it sets far stricter requirements for assisted reproduction than most people would apply to reproduction without medical help (Cavaghan, 2007).

Thirdly, the 'reasonable welfare standard' involves an intermediate position between the other two. This is the view that for (assisted) reproduction to be justified, the child to be must have a reasonable chance of an acceptable quality-of-life. On this account, the contra-indication is a high risk of serious harm (in the sense of a seriously impaired quality-of-life) (Arras, 1990; Pennings, 1999; de Wert, 1999; Benatar, 2006). The standard requires that both the magnitude of the expected harm and the probability of the harm actually occurring, i.e. the harm/probability ratio, are included in the assessment. In our view, this third standard best balances both the 
welfare of the child and the importance people attach to having children of their own. Having a 'reasonable chance of an acceptable quality of life' is intentionally vague: it is a rule-of-thumb test, to be assessed case-by-case (Glover, 2006). Applied to PGD for mtDNA disorders, the standard entails that embryos not having a high risk of serious harm are eligible for transfer. With regard to the 8993 mutations leading to NARP/Leigh, this means embryos without detectable mutant load and embryos with a mutant load below $60 \%$. Making reliable predictions regarding the health of the future child is difficult for embryos carrying a mutant load in the grey zone (60-90\%). Whether embryos in the grey zone run a high risk of serious harm is therefore debatable. With regard to the m.3243A>G mutation leading to MELAS and the private point mutations, embryos without a (detectable) mutant load would in any case be eligible, whereas there may be discussion about transferring embryos with a low mutant load. Although implying a risk of serious harm, it seems reasonable to assume that this would not amount to a high risk. Nevertheless, transferring such embryos is at the margins of acceptability (Arras, 1990). We regard this as a borderline case about which reasonable people can have different opinions.

Sex-selective PGD for homoplasmic LHON, finally, raises the question of how a $10 \%$ lifetime risk of serious visual impairment should be assessed. Most people will agree that becoming blind is not so serious that it would make a life not worth living. From the perspective of the reasonable welfare standard, however, this is not enough. The question is whether the future child can be expected to have an acceptable qualityof-life. The loss of sight, certainly when it occurs at a relatively young age, constitutes an important harm. This is not just a matter of living without the joy of seeing. The impossibility to see will also hamper daily activities and affect the quality-of-life. As a 'general purpose means', sight is valuable to nearly any plan of life (Buchanan et al, 2000). Nevertheless, blind persons do usually have happy and fulfilling lives. Moreover, the impact of blindness on the quality-of-life may also vary according to whether society has made successful efforts to remove practical impediments to social participation of the blind (Glover, 2006). As these considerations show, it is a matter of debate whether acquired blindness constitutes a 'serious harm' in the sense of a contra-indication under the reasonable welfare standard. If not, PGD for LHON is acceptable as a way of reducing but not avoiding chances that the future child will at some age become blind. However, if the expectation of acquired blindness is seen as a contra-indication, a further issue involves assessing the precise magnitude of the risks. The $50 \%$ for boys certainly constitutes a high risk, but what about the $10 \%$ for girls? Does this still amount to a high risk of serious harm? It is obvious that also this allows for different views and answers. 
An additional question regarding PGD for LHON is whether it is acceptable to transfer a female embryo that, since mtDNA is always maternally transmitted, will be confronted with the same difficult reproductive decisions as her parents (provided she has a wish for a child). After all, even when she will not be clinically affected herself, her offspring will be at risk. Although decisions indeed may be tough, this will not result in an unacceptable quality-of-life and therefore could not be a convincing argument against replacement. Besides, ICSI is an accepted form of assisted reproduction as well, also in cases where this is known to transmit the patient's infertility to his sons (de Wert, 1998).

A final issue regarding the professional responsibility to take into account the welfare of the child is whether the medical team should take stock of parental intentions. Would it matter for their decision-making about whether or not offering PGD if a couple would try to have a child anyway? Or if the couple would only consider reproduction through the less risky route (with regard to the child's health) of IVF/PGD? What if natural reproduction is simply not possible for them because of a fertility problem? Doctors should of course not stop asking themselves whether all things considered it would be acceptable for them to offer PGD. The benefits must be proportional to any health risks involved. But the mere fact that certain applications of IVF technology may lead to a child with a less than complete health does not render these application unacceptable from the point of view of the reasonable welfare standard. For instance, assisted reproduction is also offered to human immunodeficiency virus (HIV) seropositive women (both fertile and unfertile), leaving a vertical transmission rate between 1 and $2 \%$ even when all prophylactic steps are taken (Pennings, 2003; Volmink et al, 2007).

We conclude that the reasonable welfare standard (according to us the best defendable account of how the welfare of the child should figure in decisions about (assisted) reproduction) does not dismiss risk-reducing PGD for mtDNA-disorders beforehand.

\subsubsection{PGD for mtDNA disorders violates the 'right' not to know}

A second objection could be that transferring embryos with a certain mutant load leads to children being born with a positive test result for being at risk of developing a mitochondrial disease for which no effective treatment or means of prevention exists. That would seem to be in violation of the widely (but not universally) endorsed presumption that children should not be tested for late onset diseases. The exception to this is when testing offers medical benefit for the child. This would be the case when treatment is available (Clarke, 1998; de Wert, 1999; Borry et al, 2006; Steinbock, 2007). The rationale behind this position is that genetic testing would 
burden children with information that could eventually be harmful to them. It would also effectively deprive them of making their own autonomous decisions about whether or not to be tested at some later age. In other words, the child is deprived of its 'right' not to know. The same reasoning would also apply to prenatal testing. For instance, it has been argued that prenatal testing for Huntington's disease is only acceptable if the woman agrees to a termination of pregnancy in case of an adverse test result (e.g. Laurie, 1999; de Wert, 1999; Pennings et al, 2003). Does the argument also apply to PGD for mtDNA disorders? Does it provide a valid argument for not transferring embryos with (some) mutant load in cases where no unaffected embryos are available after IVF?

Although international and professional guidelines take a position against predictive testing of minors for untreatable late-onset disorders, there has been debate on this topic for years. Some argue that knowledge of its genetic status may be a psychomedical benefit for the child (Robertson and Savulescu, 2001; Rhodes, 2006). Others point to the fact that there are insufficient empirical data on the psychosocial consequences and impact of predictive testing in minors (Evers-Kieboom, 2006; Duncan and Delatycki, 2006). A recent study by Duncan et al (2007) suggests that there may even be benefits of genetic testing of young people for Huntington's disease (although this study was restricted to those aged 15 years and older). Clearly, this second possible objection against PGD would not be endorsed by those who do not categorically oppose genetic testing of minors for untreatable late-onset disorders. But what should we think about the transfer of embryos with some mutant load if we would depart from the majority view of not testing children for late onset disease unless clear medical benefits exist? Clearly, no adequate treatment for mtDNA disease is currently available and the child is provided with knowledge it might have preferred not to know.

Two comments should be made. First, the child would not exist but with the knowledge of being at risk. Even if we, strictly speaking, regard the transfer of an embryo at risk as a violation of the 'right' not to know, one should ask what is in the interests of the child and what would have been the alternative. Clearly, the alternative would have been non-existence (as the embryo would have been deselected). The main question is whether knowledge of being at risk will have such an impact that the child will not have an acceptable quality-of-life. It is implausible that this will be the case. After all, contrary to carrying the causative mutation for Huntington's disease, carrying a low mutant load is likely to have little health impact. Otherwise, it would not have been reconcilable with the contra-indication that was formulated above: avoiding a high risk of serious harm. Secondly, suppose we would 
regard the transfer of an embryo with a residual risk as an insurmountable violation of the 'right' not to know. Then, it still does not follow that performing PGD is unacceptable. It only sets limitations on the transfer policy: only mutation-free embryos (thus probably leading to disease free offspring) are eligible for transfer. Another option may be to withhold information from the couple about the precise amount of mutant load of the transferred embryo. The future child then would only know that he/she carries no or at most a small amount of mutant mtDNA. However, determining what still constitutes a small amount of mutant load is related to personal values about acceptable risk. Parents probably (and appropriately) desire to be more involved in the decision-making process regarding embryo transfer.

\subsubsection{PGD for mtDNA disorders is too complex for parents to decide}

A fourth possible objection has to do with the aim of counseling in reproductive genetics. Counseling intends to provide couples at risk of transmitting a genetic disease with information in order to facilitate well-informed reproductive choices. Decisions concerning reproduction are pivotal for people's sense of identity and for the realization of their life plans. Enhancing opportunities for reproductive autonomy is therefore rightly seen as a driving value in this field (Robertson, 1994; Boivin and Pennings, 2005; Cavaghan, 2007). However, with regard to PGD for mtDNA mutations, one may wonder whether the decisions are too difficult for them to handle.

Although testing for mtDNA disorders may have some predictive value, interpreting the results is often full of uncertainties. Starting an IVF/PGD procedure may lead to embryos with intermediate mutant loads of which the precise implications are unclear. Starting a new IVF-cycle in the hope of obtaining embryos with no or a lower mutation load is an option, but chances exist that even after several tries only affected embryos are available. Even in cases where the transfer of an embryo at risk would not be at odds with the reasonable welfare standard, the couple still faces a challenging choice. They want a child of their own, but the unavoidable price is accepting at least a low or moderate risk that this child will have a more or less compromised health.

We do not deny that these are difficult decisions, involving the processing and balancing of different types of information. Nor do we deny that empirical studies have shown that in general people are not very good at handling statistics (Schwartz, 2004). We also agree that there are many other pitfalls surrounding the ideal of enhancing autonomous decision-making, including emotional stress or misplaced trust that whatever doctors offer must be good. However, none of this is limited to decision-making in the context we are considering. When deciding, e.g. whether to 
have chemotherapy and which regimen to follow, there may also be several kinds of uncertainty to be dealt with, whereas time constraints, illness and stress are complicating factors. No matter how difficult such decisions may be, this is never taken as a ground for not offering chemotherapy. We see no reason why this should be different here. Moreover, decision-making for PGD has the (relative) advantage that the choices can be made before the IVF procedure is started. This does not take away the complexity of the decisions, but at least it leaves some time and scope for reconsideration and reflection.

Depriving competent couples at risk from the option of IVF/PGD because the decisions are too complex amounts to a form of paternalism that would be hard to justify. Since decisions like these will be very much related to the personal values of the couple, there is no good reason for thinking that doctors or society would be better placed to make the decisions. Instead of curbing autonomy, we plea for enhancing autonomy as much as possible and facilitating reproductive choice, which means that good counseling becomes even more important (and this is a topic that needs continuous concern, as time is a scarce resource in daily clinics).

\subsubsection{PGD for mtDNA disorders ignores available alternatives}

A further objection might be that there are alternatives allowing the couple to have a healthy child. They could opt for IVF using donor oocytes or apply for adoption. Should this be a reason for not offering PGD, or at least for not transferring any embryos at risk? The question is whether the available options constitute real alternatives. A first problem is that their availability may in reality be very limited. Not only generally speaking, but more specifically so for the couples involved. Donor oocytes are very scarce, but even more so when maternal relatives are not suitable donors as they may also be at risk of transmitting the same mutation. Adoption procedures are not just lengthy, complicated and expensive, but often also exclude couples with a less than perfect health.

However, for many of the couples involved, there is a more fundamental reason why these options would not count as real alternatives. They do not just want a child, they want a child of which they are the genetic parents together. Of course they also want this imagined child to be completely healthy, but should that be impossible, the alternative for them would be a less healthy child of their own, rather than a child with which they would not both be genetically related. As there are limits to the risks they are willing to take, risk-reducing PGD provides them with a valuable reproductive option, for which only natural reproduction followed by PND would be an alternative. By choosing for PGD they may reassure themselves that they did all that was 
reasonably possible to avoid the birth of an affected child. In the future, things may change if (pro)nuclear transfer were to become available as a way of allowing couples at risk of mtDNA mutations to have non-affected children of their own (Brown et al, 2006). At present, however, that is still a remote option, mainly because of safety concerns that have to be solved first. Besides this, nuclear transfer raises several other ethical questions.

We may of course question whether the desire for a genetically related child should be taken as important enough to justify PGD for mtDNA mutations without even considering donor IVF or adoption. It has been argued that if our genetically related children will suffer some disease, perhaps we should have genetically unrelated children without these diseases' (Savulescu, 1999). This is certainly a perspective that some couples at risk of transmitting an mtDNA mutation will find convincing. Obviously, it is important that counselors discuss this as a possible way of looking at the situation. But to suggest that couples ought to take this view is problematic. It fails to acknowledge how deeply entrenched, both biologically and culturally, the ideal of having genetically related children seems to be. One need only to look at how current practices of assisted reproduction are built around this very ideal. For instance, in cases where the male partner is at risk of transmitting a genetic disease, 'regular' PGD is offered without urging the couple to try donor insemination first, even though that is a simpler, cheaper and more efficient procedure to have a healthy child. It seems unfair that only couples at risk of transmitting an mtDNA mutation would have to revise their preferences. Nor would the fact that in their case genetically related children may all be affected amount to a sufficient reason for denying them the option of PGD. To suggest otherwise betrays allegiance to the 'perfectionist' standard of maximum welfare that would be difficult to maintain consistently. As we have argued, short of a high risk of serious harm, the health prospects of the future child are not a good reason for withholding IVF/PGD.

\subsubsection{PGD for mtDNA disorders leads to an unacceptable wastage of human embryos}

Yet another possible objection may hold that PGD for mtDNA mutations leads to an unacceptable wastage of human embryos. There are different ways of construing this argument. If it is not to be understood as a rejection of all practices where human embryos are wasted (including IVF and natural intercourse), the issue must be about proportionality rather than acceptability per se.

PGD for mtDNA mutations is likely to consume far more embryos than 'regular' IVF/PGD. Although this is not true for sex-selective PGD for LHON (where on average half of the embryos will be available for transfer), it is true where 
heteroplasmic mutations are concerned. More embryos may have to be rejected because of an unacceptably high level of mutant load than because of Mendeliantype mutations in 'regular' IVF/PGD. As this will more often lead to an aborted procedure, or to having a new try, the net result is a greater wastage of embryos. To put this in perspective, however, we refer to the practice of PGD for HLA-typing, where more than $80 \%$ of the embryos are not suitable for transfer (de Wert et al, 2007). Furthermore, wasting embryos can only be proportional when it is for a sufficiently valuable end. So what about the end of PGD for mtDNA mutations (here again including homoplasmic LHON)? How does mere risk reduction compare to the ends of IVF (allowing the infertile to have children) or 'regular' PGD (allowing couples at risk to have unaffected children)? This will of course also depend on the amount of risk reduction that is achieved. As long as this is a considerable reduction, it would seem quite beside the point to suggest that this end would somehow be less important than those of other applications of assisted reproduction. Moreover, once a couple has been fully informed about the risks and burdens of IVF/PGD and they still wish to proceed, then this can be considered as an indication of the seriousness of the disease and the importance they attach to the reduction of the health risks (Amor and Cameron, 2008).

\subsubsection{PGD for mtDNA disorders sets us on a slippery slope}

Finally, it can be objected that we are stepping on a slippery slope when we apply PGD for mtDNA disorders. According to this argument ('slippery slope' or 'thin end of the wedge'), introducing or accepting a technology or application (A) that in itself may not be morally problematic or unacceptable, would still be problematic or unacceptable if doing so would make it impossible (logically or empirically) to avoid the subsequent introduction or acceptance of another technology or application (B) that would be morally unacceptable (van der Burg, 1992; Burgess, 1993; McGleenan, 1995). In the logical version of the slippery slope, accepting A deprives one of valid arguments for rejecting $B$. The empirical version consists of the prediction that accepting $A$ will lead to a climate of acceptance towards $B$ as well. In both versions, the message is that anyone who regards $B$ as undesirable should reject $A$ (Lamb, 1988; de Wert, 2005). With regard to the acceptability of PGD for mtDNA disorders, at least three different slippery slopes are imaginable.

\section{Sliding to increasingly higher risks}

Will accepting embryos with a low mutant load (thus with small risks) as eligible for transfer unavoidably lead towards accepting the transfer of embryos with much higher risks? As in all slippery slope arguments, the first question is whether the imagined position at the bottom end of the slope is indeed to be avoided. The answer 
has already been given: IVF/PGD is unacceptable if there is a high risk that the resulting child will suffer serious harm. The second question is about the inevitability of sliding down. Does allowing the transfer of embryos at a low level of risk leave us without valid arguments against allowing high-risk transfer? As we have indicated, 'high risk of serious harm' is not a clear-cut criterion. There will be borderline cases that allow for different views and interpretations. However, that does not amount to saying that a material distinction between high and low risks cannot be made. Nor is it plausible to think that the transfer of embryos at low risk will create a climate in which gradually larger and larger risks are accepted, until 'high risk of serious harm' is no longer seen as a limiting criterion.

By the way, the transfer of an embryo with some mutant load would not be the first departure from the 'norm' of not transferring embryos at risk. Ever since the introduction of PGD, sex-selective transfer of female embryos has been standard practice for several X-linked diseases (such as Duchenne muscular dystrophy) where mutation analysis was or is not (yet) available (or is considered to be disproportionally time-consuming). Half of these female embryos will be carriers. Though generally healthy, carriers are to some extent at risk of showing relatively mild symptoms of the disease. So if there is a slippery slope here, a first step on that slope has already been set long ago. There is no indication that the long-standing practice of selecting low-risk carrier embryos in PGD for X-linked diseases has led to a greater acceptance of transferring embryos at a higher risk. It is, however, conceivable that in clinical practice the absence of embryos with a low mutant load may lead couples to ask for transfer of embryos with higher mutant loads. But this can be countered by making clear agreements with the couples when considering clinical application.

\section{Sliding to increasingly smaller reductions of risk}

IVF/PGD for mtDNA disorders aims at least to achieve a (considerable) reduction of reproductive risk. It is thought that by discarding embryos with a high or intermediate mutant load, the risk of a severely affected child can be considerably reduced. In homoplasmic LHON, sex-selective PGD in comparison to natural reproduction reduces the lifetime risk of blindness from 30 to 10\% (Spruijt, 2007). Will accepting risk reduction as an alternative end of PGD set us on a slippery slope towards eventually accepting much lower percentages of risk reduction? Here again, the first question is whether the dreaded position at the foot of the slope would indeed be morally unacceptable. In this case, the question is what magnitude in risk reduction is sufficient for IVF/PGD to be offered (Amor and Cameron, 2008). This leads us back to the issue of proportionality. As the benefits of any medical procedure must be 
balanced against the material and immaterial costs, increasingly more marginal benefits will end up rendering risk-reducing PGD disproportional. The cost side of this equation would also have to include the risks and burdens of IVF, potential long-term risks of embryo biopsy for the health of the child, and the use of human embryos. There will of course be debate and different opinions about this, leading to various accounts of where the limit of proportionality must be drawn. But does this put us on a slippery slope? The logical version of the argument does at least not seem to apply, as the very concept of proportionality gives us a tool (albeit a rough one) for distinguishing between acceptable and non-acceptable applications. Neither is the empirical version convincing. In view of the costs and burdens of IVF and PGD, not many couples will request IVF/PGD for increasingly more marginal reductions of risk. The medical team should decide on a case-by-case basis what they still regard an acceptable amount of risk-reduction, also depending on the specific mutation and the wishes and circumstances of a specific couple.

\section{Sliding to increasingly milder conditions}

Most mtDNA mutations show an incomplete penetrance and a variable expression. This means that not all persons carrying the mutation will develop clinical symptoms and that in those who do, the severity of symptoms varies even if having the same mutant load. Will accepting PGD for such conditions set us on a slippery slope towards also accepting PGD for conditions with very low burdens of disease? If there is a slippery slope here, PGD for mtDNA disorders is not the first step. At least not in countries, such as the UK, where-on a case-by-case basis-PGD for hereditary cancers such as BRCA I/II is now accepted. Far from being low risk or mild conditions, these disorders also show an incomplete penetrance and a variable expression, in addition to a late time of onset and (limited) therapeutic options. To the extent that slippery slope concerns are about PGD for increasingly more marginal benefits, we are again dealing with the proportionality issue. In this connection, however, there may be a further concern. PGD for disorders with a lower penetrance and a variable expression may set us on a slippery slope towards regarding smaller and smaller health problems as a reason for genetic testing and prevention. Those living with these conditions may feel compromised. Or couples at risk may experience (social) pressure to make use of genetic technology. If this is where we are heading, this is morally problematic. Nevertheless, it does not provide sufficient reasons to prohibit PGD for mtDNA mutations (or hereditary cancers). After all, accepting or not accepting these new applications of PGD will be at most a relatively minor factor in a much wider configuration of social and cultural causes affecting such a change of climate. 


\subsection{Conclusion}

We have discussed the pros and cons of introducing applications of PGD that may only reduce rather than eliminate reproductive risk. Specifically, we examined the case of PGD for mtDNA disorders, such as NARP/Leigh, MELAS, private mtDNA mutations and LHON. The main arguments in favour of PGD are that it offers couples at risk the opportunity of reproducing while (considerably) reducing and in some cases eliminating their chances of having a severely affected child. Introducing PGD for these conditions is not only beneficial in the sense of contributing to positive health outcomes, but also enhances the reproductive autonomy of the couples involved. Further benefits for patient care in general can also be expected, as PGD will provide new possibilities for the scientific study of mtDNA genetics by using embryos not selected for transfer.

Potential objections are manifold but, as our discussion has shown, none of them supplies compelling reasons to regard risk-reducing PGD as unacceptable. Notwithstanding the acceptability of PGD for mtDNA disorders, our discussion has made clear that introducing this new form of PGD will raise complex issues in the context of determining conditions for its responsible use. Although the avoidance of a high risk of serious harm is the lower limit, an important point for discussion once a couple requests $P G D$ is how much additional effort should be made to further reduce the health risks and to minimize harm. For example, how many IVF/PGD cycles could (or should) be done before an embryo at risk is transferred? And what should be the cut-off points above which embryos are not considered eligible for transfer? Clearly, these and other relevant questions require further interdisciplinary debate. 



\section{Chapter 4}

\section{Preimplantation genetic diagnosis for mitochondrial DNA disorders: ethical guidance for clinical practice}

Bredenoord AL, Dondorp WJ, Pennings G, De Die-Smulders CEM, Smeets HJ, de Wert G.

European Journal of Human Genetics 2009 Dec;17(12):1550-1559 


\begin{abstract}
Although morally acceptable in theory, preimplantation genetic diagnosis (PGD) for mitochondrial DNA (mtDNA) disorders raises several ethical questions in clinical practice. This chapter discusses the major conditions for good clinical practice. Our starting point is that PGD for mtDNA mutations should as far as possible be embedded in a scientific research protocol. For every clinical application of PGD for mtDNA disorders, it is not only important to avoid a 'high risk of serious harm' to the future child, but also to consider to what extent it would be possible, desirable and proportional to try to reduce the health risks and minimize harm. The first issue we discuss is oocyte sampling, which may point out whether PGD is feasible for a specific couple. The second issue is whether one blastomere represents the genetic composition of the embryo as a whole - and how this could (or should) be investigated. The third issue regards the cut-off points below which embryos are considered to be eligible for transfer. We scrutinize how to determine these cut-off points and how to use these cut-off points in clinical practice - for example, when parents ask to take more or less risks. The fourth issue regards the number of cycles that can (or should) justifiably be carried out to find the best possible embryo. Fifth, we discuss whether follow-up studies should be conducted, particularly the genetic testing of children born after IVF/PGD. Finally, we offer the main information that is required to obtain a truly informed consent.
\end{abstract}




\subsection{Introduction}

When applying preimplantation genetic diagnosis (PGD) for mitochondrial DNA (mtDNA) disorders, interpretation of the test results may be difficult and it is conceivable that in some cycles only affected embryos are available for transfer. Instead of enabling parents to have a healthy child, PGD then may only contribute to a reduction of reproductive risk by selecting the embryos with the highest probability of leading to a healthy child. Earlier (Bredenoord et al, 2008a;b), we discussed the pros and cons of PGD for mtDNA disorders, concluding that despite the drawbacks no convincing moral arguments exist to regard risk-reducing PGD as unacceptable. Nevertheless, several ethical questions arise when considering application in clinical practice. These specific ethical questions are generated by the characteristics of mtDNA genetics (such as heteroplasmy, which is a mixture of normal and mutant mtDNA, the level of which can differ among tissues). When a couple asks for PGD for an mtDNA mutation, is it morally justified to honor their request and to (experimentally) offer them PGD? Or is this still premature, taking into account the risks and uncertainties? After all, the reasonable welfare standard, which we consider to be the best way to take into account the welfare of the child, allows PGD for mtDNA mutations insofar, as there is no 'high risk of serious harm' to the future child. This standard entails the view that for assisted reproduction to be justified, the child to be must have a reasonable chance of an acceptable quality of life (de Wert, 1998; Pennings, 1999; Bredenoord et al, 2008b). What are the implications of this standard for determining the heteroplasmy levels (the cut-off points) below which embryos are considered to be eligible for transfer? Does sufficient knowledge exist about whether the analyzed cell(s) represents the entire embryo? And to what extent should one search for better embryos, for example by starting another cycle of in vitro fertilization (IVF)?

In this chapter, we will examine the questions that may successively arise when PGD for mtDNA disorders is considered for application in clinical practice. The central question of this paper is: which moral requirements have to be met before applying PGD for mtDNA disorders in clinical practice? We will not offer a clear-cut recipe, but we aim to present the ingredients, the points to consider when offering PGD for mtDNA mutations.

Three further preliminary remarks should be made. Firstly, little experience exists with PGD for mtDNA mutations (thus far only two applications have been reported) (Bickerstaff et al, 2001; Steffann et al, 2006). Some considerations may therefore be to some extent speculative. This is unavoidable if one aims to practice ethics proactively and this is a reason why we discuss several scenarios. Secondly, we will in 
this paper only consider blastomere biopsy, as PGD on blastomeres currently has a higher efficiency and accuracy than the other approaches (Briggs et al, 2000; Dean et al, 2003). Thirdly, if PGD would indeed be offered, good reasons exist to embed the first applications of PGD for mtDNA mutations in a scientific research protocol. This is the most efficient way to obtain systematic data and to increase knowledge in mtDNA genetics. A further advantage is that it guarantees that patients are sufficiently aware that they are enrolled in an experimental treatment. Obviously, the rarity of some mtDNA mutations may limit the feasibility of clinical trials. In such cases, efforts should be made to conduct (international) multi-centre trials.

\subsection{Three categories of mutations}

Most ethical difficulties in mitochondrial clinical practice are related to interpretation and segregation of heteroplasmy. PGD for homoplasmic mutations (that is, only mutant mtDNA is present) such as Leber Hereditary Optic Neuropathy raises different types of ethical questions (Bredenoord et al, 2008b). This paper is therefore restricted to the heteroplasmic mutations. The reliability of PGD for those mutations will depend on whether the specific mutation shows (Bredenoord et al, 2008b; Poulton and Turnbull, 2000):

1. a close correlation between the mutant load (that is, the ratio of mutant to normal mtDNA) and disease severity;

2. a uniform distribution of mutant mtDNA in all blastomeres;

3. no change in mutant load with time (both prenatally and postnatally).

We will in this chapter refer to three categories of mutations.

Category I regards stable mutations with a predictable outcome. These mutations fulfill the three criteria above (White et al, 1999a;b; Dahl et al, 2000). The main examples are the mutations m.8993T $>G$ and m.8993T>C, leading to the neurodegenerative diseases NARP (Neurogenic muscle weakness, Ataxia, Retinis Pigmentosa) and Leigh syndrome. Both mutations have a strong genotypephenotype correlation and show very little tissue-dependent or age-dependent variation in mutant load (White et al, 1999a;b; Dahl et al, 2000). For these mutations, in general, symptoms seem to occur above $\sim 50 \%$ mutant load and the frequency and severity increases with increasing mutant load (White et al, 1999a). Patients with severe NARP syndrome generally have mutant loads above $70-80 \%$, although some exceptions have been reported as well (White et al, 1999a). Patients with severe Leigh syndrome generally have mutant loads above 80\% (White et al, 1999a). Nevertheless, a twofold cautiousness is required. Firstly, cautiousness regarding the exact cut-off levels, as statistical variation of the mutation percentages is rarely 
supplied and depends highly on the technology used and the number of patients tested. Secondly, cautiousness regarding the interpretation of 'seriousness'. Although some general outlines may be given, what exactly constitutes 'serious symptoms' is something to be assessed case-by-case, depending on the specific circumstances. Although the m.8993T>C mutation (Leigh) is generally considered to be clinically milder than the m.8993T>G mutation (NARP) and the threshold for disease expression seems to be slightly higher, we discuss these mutations here together as the nucleotide 8993 mutations. We presume that also during embryonic and fetal development, tissue and time-dependent variation in mutant load is very limited. Above $80 \%$, the child is very likely to be affected. There is a gray zone between 50 and $80 \%$ mutant load, especially for the m.8993T>G NARP mutation (White et al, 1999b; Tatuch et al, 1992; Ciafaloni et al, 1993). This zone is 'gray' in the sense that there is a chance of developing symptoms, but the frequency and seriousness are not exactly known, and in the sense that precise numbers are lacking because of technical variables. One may reasonably assume that an embryo with a mutant load below $50 \%$ is very likely to result in a healthy child.

Category II are mutations, which are unstable in time with a non-uniform tissue distribution and with no reliable genotype-phenotype prediction on the basis of mutant load. They do not fulfill the criteria above (Poulton and Turnbull, 2000; Chinnery, 2002). An example of this is the m.3243A>G mutation leading to MELAS, which is one of the most common mtDNA mutations. The clinical expression of this mutation is very variable, the classical presentation being the combination of mitochondrial myopathy, encephalopathy, lactic acidosis and stroke-like episodes, whereas in other families only diabetes mellitus and deafness are found. However, not only interfamilial, but also intrafamilial, variation may be huge and is only partly related to mutation load. In general, symptoms may occur above $30 \%$ and the frequency and severity increases with increasing mutation load (Chinnery et al, 1998). However, exceptions occur and prognostic predictions on the basis of mutation load are not reliable for individuals (Chinnery, 2002).

Category III contains mutations, which are private or family-specific point mutations and for which no additional information is available. Insufficient evidence exists to decide whether they fulfill the criteria mentioned and allow reliable PGD.

\subsection{Feasibility and representativity}

\subsubsection{Feasibility: oocyte sampling?}

Oocyte sampling is an option to be considered to answer the question whether IVF and PGD will be feasible (that is, would have a fair chance of success) for a 
particular couple. After a superovulation cycle, all the oocytes of the woman (on average 10-12 oocytes after ovulation induction) are taken and analyzed to assess the chance that eventually embryos with no or a low-mutant load are available (Poulton and Marchington, 2002). For the 8993 mutations, an atypical (skewed) segregation of mutant load among the oocytes may be expected (Dean et al, 2003; Blok et al, 1997). This would be favorable, because this increases the chances of obtaining low or zero-mutant embryos (although the chance of obtaining high mutant load embryos will be increased as well). For the m.3243A>G mutation, a more continuous distribution of mutant load may be expected (Brown et al, 2001). When it would become clear beforehand that the chance of obtaining oocytes with no or a very low-mutant load is negligible, there seems to be no use in starting an IVF/PGD procedure. For those ascribing high moral value to embryos, an advantage of oocyte sampling is that unnecessary creation and destruction of embryos is avoided. Another advantage is the (material and immaterial) costs that may be saved, as there will be no fertilization and further PGD analysis (and subsequently no complex decision making regarding which embryo to transfer). However, these advantages only apply for women with oocytes containing high mutant loads. A major disadvantage for women for whom PGD does seem to be feasible is that they have to undergo a novel cycle; their analyzed oocytes are lost and thus cannot be used to establish a pregnancy. Therefore, we propose to sidestep oocyte sampling and proceed directly to IVF and PGD.

\subsubsection{Representativity: a uniform distribution?}

Before PGD can be considered for an mtDNA mutation, it is important to ascertain that the proportional levels of mutant to wild-type mtDNA quantified in the biopsied cell are representative of the levels in the embryo as a whole (Dean et al, 2003). This issue of representativity is of utmost importance from a moral point of view: would there not be a uniform distribution, then the cell(s) taken for analysis do(es) not represent the genetic composition of the whole embryo. This may result in the birth of a severely affected child. Data of neutral polymorphisms in mice and human, and data regarding the 8993 mutations indicate that the intercellular variation of mitochondrial heteroplasmy is fairly small among most preimplantation embryos (Steffann et al, 2006; Dean et al, 2003). Data are limited for the m.3243A>G mutation. Blastomere representativity could be investigated at two stages: preclinically (IVF/PGD without the intention of embryo transfer) and clinically (IVF/PGD with the intention of embryo transfer). What would be the best route to ascertain this? 


\section{Preclinical route}

In a preclinical route, embryos carrying an mtDNA mutation could be created and dissected to find out whether all blastomeres carry the same amount of mutant mtDNA. If it turns out to be the case, this can for the moment be taken as sufficient proof of blastomere representativity for the mutation in question. The only possibility to obtain embryos carrying a specific mtDNA mutation would be to create them by means of IVF. This requires donation of gametes from both a male and a female donor, whereby the oocyte donor should carry the mtDNA mutation in question.

This leads, firstly, to an ethical assessment of oocyte donation for research purposes and, secondly, to an ethical assessment of creating embryos for research purposes. Given the burdens and risks of the procedure, oocyte donation is morally sensitive. We, nevertheless, consider it to be morally acceptable, provided the risks are minimal, the donor is sufficiently informed about the risks and discomforts, and voluntariness is safeguarded (Mertes and Pennings, 2006; Pennings et al, 2007). In most countries creating embryos specifically for research is both legally prohibited and morally rejected, particularly because of the contested moral status of the embryo. Many would only accept the creation of embryos in the context of a parental project. But what would be wrong with creating embryos in the context of preparing for a possible parental project? The final goal would be to enable couples at risk to conceive healthy children. Although those first embryos will not be used directly for a pregnancy, subsequent embryos will be used, provided the results are encouraging. Although in some cases an extra step is necessary, the ultimate goal remains the conception of children. It cannot be maintained that this is morally very different from the current IVF practice, with its implied acceptance of creating and discarding surplus embryos (Sandel, 2007). Once one accepts the creation and killing of embryos to benefit infertile people with a child-wish, it seems questionable to argue that the creation and killing of embryos for research (which eventually may benefit ill people) is condemnable (Devolder, 2003; Dondorp and de Wert, 2005).

\section{Clinical route}

Another way of obtaining more evidence for embryo representativity could be at the beginning of a clinical application of IVF/PGD. Two approaches are conceivable.

A first approach may be to start an IVF-cycle and to examine two cells of each embryo available (usually 10-12 embryos are available). This may sufficiently prove whether there is a uniform distribution of mutant mtDNA in all blastomeres. If there would be a problem with an uneven segregation, that is, large differences in mutant load, this would probably become clear (although this cannot be stated with certainty, as there will always be a limited number of samples). However, two-cell biopsy is controversial, as current data indicate that it may impede success rates (Cohen et al, 
2007; Combelles, 2008; Goossens et al, 2008). Cohen et al argue that 'this [two-cell biopsy] approach should be reserved for cases in which diagnostic accuracy is considered paramount, more important than embryo implantation' (Cohen et al, 2007). With regard to the mtDNA mutations, diagnostic accuracy actually is more important than implantation. A two-cell biopsy therefore would be justifiable, at least in the first applications: if the mutant load can be reliably determined, one can return to single cell biopsy, and if this is not the case, one should stop offering PGD.

A second approach would be to test a single cell of each embryo. Subsequently, all remaining blastomeres of those embryos with a high mutant load are analyzed within 24 hour (to be able to use this information before implantation). If it turns out that the mutant load for all tested embryos is similar in all blastomeres, this indicates that one blastomere represents the genetic composition of the embryo as a whole. This second approach may be conducted to avoid a two-cell biopsy. It can also be used to determine whether one or two blastomeres should be used for the second cycle.

Both preclinical and clinical investigation may thus indicate whether PGD is reliable. What would be the best approach? If the 'clinical' route indeed provides sufficient evidence, then this route is preferable over the preclinical route. After all, the most important goal - assuring that the genetic test is reliable - can be met and a pregnancy would still be within the possibilities. This fits both the interests of the woman (that is, a healthy pregnancy) and the principle of proportionality (that is, the risks, costs and burdens of a procedure balanced against the expected outcome). As the clinical route is likely to consume fewer embryos than the preclinical one, the clinical route is also preferable from an embryo-saving perspective.

\subsection{Determination of cut-off points}

A cut-off point is the threshold of mutant load above which no embryos are considered for transfer. If only embryos above the threshold are found, further options are limited to either trying again by starting a new IVF/PGD cycle or deciding to stop trying PGD. It is important to stress that a cut-off point figures as the lower limit. It points out what amount of risk is still considered acceptable. Searching for embryos with a lower mutant load is not morally neutral. When opportunities exist to improve the outcome, that is what one should aim at - taking into account other morally relevant aspects as well. This implies that for every clinical application of PGD for mtDNA disorders, it is not only important to be sure that helping this specific couple would be justifiable in view of the reasonable welfare standard, but also to consider to what extent it would be possible, desirable and proportional to try to further reduce the health risks by adapting procedures or trying another cycle. Of course, these are questions both for the medical team and the couple. 
For all mtDNA mutations, health risks could be reduced substantially by determining a low cut-off point. However, the lower the cut-off points are fixed, the more embryos are discarded, and thus, the lower the 'take home baby rate'. On the other hand, the higher the cut-off points are fixed, the more embryos will probably be available. The price to be paid is an increased risk that the welfare of the child will be compromised. In the process of determining the appropriate cut-off point, both the proportionality and the welfare of the child need to be assessed. After all, the risks and burdens (material and immaterial) of IVF and PGD must stand in a reasonable proportion to the desired aim, which is the conception of a (healthy) child.

What, then, are the appropriate cut-off points? For the 8993 mutations (category I), levels of mutant load below $50 \%$ seem compatible with a healthy phenotype (White et al, 1999a;b; Pettman et al, 2007). A grey zone exists between 50 and $80 \%$. Whether the reasonable welfare standard also allows embryos in the gray zone to be transferred is debatable and also depends on the specific mutation. The more the mutant load is heading towards the $80 \%$, the higher the probability of (severe) NARP or Leigh symptoms. Nevertheless, a cut-off point of $80 \%$ may be justifiable as well, bearing in mind that a cut-off point is the absolute lower limit. This implies that if one takes a cut-off point of $80 \%$, physicians and parents should consider to make efforts to search for embryos with a lower mutant load.

For the $m \cdot 3243 A>G$ mutation (category II), the threshold to disease expression is not exactly known and can vary among individuals, which makes determining the cut-off point an intricate enterprise. As a mutant load below $15 \%$ does not seem to result in a severe phenotype, it seems in accordance with the reasonable welfare standard to determine the cut-off point for the m.3243A>G mutation at 15\% (Chinnery et al, 1998; 2000). Although also here a certain margin exists, embryos carrying $15 \%$ mutant load probably do not run a high risk of serious harm.

Characteristic for the group of private mtDNA mutations (category III) is that they are only found incidentally, in only a few families or even a single one, and that therefore insufficient information is available to judge if these mutations allow sufficiently reliable predictions. If more information about a specific mutation would be available, then that mutation would not fit this category any longer (it would shift to category I or II). PGD for any point mutation may be offered as long as mutant-free embryos are available and can be reliably identified for transfer. The problem of transferring embryos with some mutant load is that it is difficult to determine a cut-off point for each private mutation because of the lack of data. For some private mutations, a cutoff point may be derived through extrapolation of what is known about other 
mutations, as for example has been done for the T9176C mutation (Jacobs et al, 2005). Another option is to determine the biochemical cut-off point in a cell line system (cybrids). The rationale is that clinical manifestations are unlikely to occur if the mutation load does not lead to an enzyme deficiency. Nevertheless, as this is rather speculative, we will restrict ourselves here to category I and II, the 8993 and m.3243A>G mutations (which raises similar ethical questions as the private mutations).

The proposed cut-off points above should be regarded as provisional. They should be adjusted when more experience exists with PGD for mtDNA mutations, or when more is known about the threshold to disease expression (and for some mutations complete clarity may never be obtained because of the clinical variability and the possible changes in mutant load).

\subsection{Taking more risks?}

It is conceivable that a couple does not agree with the proposed cut-off point (which may already become clear in the pre-test counseling or during the procedure). Although the initial aim of a couple may have been to look for an embryo below the cut-off point, no suitable embryos may become available during the IVF/PGD procedure. It is conceivable that couples with a very strong wish for a child are satisfied with the transfer of an embryo with a mutant load above the determined cutoff point. This may be especially the case for couples depending on IVF anyway because of fertility problems - which broadly speaking concerns $50 \%$ of PGD users (Harper et al, 2008). Is it acceptable, then, to follow their wish? A (slightly) flexible use of the cut-off points may be defended on grounds of two considerations.

The first consideration is whether the acceptability of taking more risk depends on the number of cycles already carried out. One can imagine that a couple would be more inclined to ask for the transfer of embryos with (some) mutant load in a third or fourth cycle than when they just have started IVF/PGD. Although it is a request easily sympathized with, adhering to the reasonable welfare standard implies that a high risk of serious harm for the resulting child should be avoided in any case. The transfer of an embryo with a mutant load that is likely to cause a severe phenotype is morally unjustified. The second consideration is that some arbitrariness regarding the cut-off values cannot be avoided and that the safety margin taken can be debated, especially for couples in whose family the mutation seems to show a mild phenotype (e.g., diabetes mellitus in case of the m.3243A $>$ G mutation). How far do you need to be on the safe side? Which arguments are valid to adjust the cut-off point? Any sensible argument either directed on the percentage as such, the clinical 
interpretation or the safety margin should be discussed on a case-by-case basis by the health care professionals involved in PGD.

There may also be couples who want to take less risk or no risk at all. They, for example, only regard mutant-free embryos as eligible for transfer. This will be examined below, when we discuss the number of cycles. Another situation that may occur is that a choice has to be made between a low mutant load embryo that is morphologically of lesser quality and a higher mutant load embryo that is morphologically of better quality. Here, one has to maneuver between the futility of transferring an embryo of very poor morphology on the one hand and not exceeding the cut-off point on the other hand.

\subsection{Number of cycles}

Chances of eliminating (or at least minimizing) health risks may increase with the number of embryos. The more embryos available, the higher the chances of obtaining embryos with zero or low(er) mutant load. The number of embryos can be increased in two ways. The first involves a stronger ovarian stimulation. This could provide more oocytes and thus more embryos. Whether it is acceptable to offer a more aggressive hormone treatment to obtain more embryos has already been a point of debate with regard to PGD for translocations (Chen et al, 2005). It is quite contrary to the current tendency to mild ovarian stimulation (Heijnen et al, 2007). Nevertheless, it should not be completely ruled out, especially when the woman belongs to a low-risk group for developing ovarian hyperstimulation syndrome.

A second way to obtain more embryos is by conducting more cycles. Suppose that IVF/PGD for the m.3243A>G mutation results in one embryo with a mutant load of $12 \%$. Or, when PGD is carried out for one of the 8993 mutations, an embryo with $70 \%$ mutant load is available (being in the so-called gray zone). Is it morally justified to transfer these embryos? Or should the medical team propose to start a new cycle to find a 'better' embryo?

\subsection{Should one start a new cycle?}

In other applications of PGD, the medical team will not propose a new cycle if suitable embryos are at hand. So why would they do so here? Although one should, other things being equal, strive to minimize risks, this should also be weighed against the implications. In view of the (physical, emotional and financial) burdens of both IVF and PGD (both for the couple and for society), it can be questioned whether conducting a new cycle is justified. Many couples experience an IVF/PGD cycle as extremely stressful (Lavery et al, 2002). Furthermore, cryopreservation of biopsied embryos is not very successful, at least not at this moment (Magli et al, 2006; Parriego et al, 2007). The chance exists that only embryos in worse condition are 
obtained when one starts a second or third IVF/PGD cycle. The overall result then would be that suitable embryos were available in an earlier round but that these cannot be used anymore because they did not survive thawing. To start a new round when a suitable embryo is available is thus quite a gamble. So is this still proportional?

A reply to this may be that the proposed cut-off points are debatable at the state of knowledge. One cannot say with certainty that an embryo in the gray zone (8993 mutations) or an embryo with, for example, $12 \%$ mutant load ( $m .3243 A>G$ mutation) will result in an unaffected child. One could therefore prefer to obtain embryos with the lowest possible mutant load as long as this application of PGD is still novel and experimental. Starting a new cycle therefore is morally acceptable. Moreover, it is shown for at least a few families that obtaining embryos without mutant load is realistic (Steffann et al, 2006). One could therefore argue that if one chooses to use a risky technology and a possibility exists to considerably reduce the risks, then physicians and the couple should at least consider to do so. One could in this light even consider to preferentially transfer male embryos to reduce the risk of transmission to next generations.

In view of our discussion above, we now may delineate a rough framework about when (not) to start another IVF/PGD cycle and which embryo(s) to transfer (we accept the general consensus regarding the transfer of one, maximally two embryos). Although it seems best in theory to look for 'the best possible embryo', in practice this may be no sinecure. The lower limit in all cases is the avoidance of a high risk of serious harm for the child. More concrete, on the current state of knowledge we have proposed to use a cut-off point of $15 \%$ for the m.3243A>G MELAS mutation. For the 8993 NARP/Leigh mutations, we have proposed to use a mutant load of $80 \%$ as the cut-off point. For many private mutations, it is impossible because of the lack of data to offer a well-founded cut-off point. For those mutations, PGD can be offered but only embryos with zero-mutant load seem eligible for transfer. Although it is morally acceptable that a couple and the medical team confer about whether or not to start a new cycle, looking for better embryos should not be seen as morally required if an embryo below the cut-off point is available. The precise number of cycles is something that should be determined on a case-by-case base, also depending on the specific mutation, the wishes of a couple, their individual chances of success, the number of effective transfers and the number of cycles allowed and reimbursed in a country. 


\subsection{Scientific research after IVF/PGD}

After the IVF/PGD procedure, not all uncertainties surrounding PGD for mtDNA mutations will be resolved. This will particularly be the case for the unstable mutations with an unpredictable outcome (that is, category II, such as the m.3243A>G mutation). Residual uncertainties mainly regard whether the mutant load has changed in time and whether there is a uniform distribution of mutant mtDNA in all blastomeres. Various types of post-procedure scientific research may contribute to the further clarification of these uncertainties.

The first type of scientific research concerns analysis of the embryos that will not be transferred. Probably, the most interesting aim of this research is to check the segregation of mutant load between the blastomeres. To the extent that this would provide an extra safety check relevant for further applications, this type of research is recommendable. It is desirable that the medical team asks the parents to donate their affected embryos for scientific research (as is standard procedure in other applications of PGD).

Another option to check for reliability of the PGD test regards prenatal diagnosis (PND), using CVS or amniocentesis during the pregnancy. However, as we cannot do justice to the complex issue of PND for mtDNA disorders within the scope of this chapter, and as debate is possible about the added value of PND for mtDNA disorders, we will not further discuss this option. Further interdisciplinary debate on whether (and if so, under what conditions) PND should be offered after PGD is desirable.

\subsection{Testing children}

We will focus on a third type of scientific research, that is, the genetic testing of the children born as a result of IVF/PGD for an mtDNA mutation. The main goal would be to validate the PGD procedure by ascertaining that the genetic diagnosis made at the embryonic stage is a reliable predictor of the health of the resulting child. Clearly, periodic clinical examinations could also add to this aim, but only after years and not as clearly as a genetic test could do. The danger is that PGD for mtDNA mutations would be introduced into the clinic and would turn out to be unreliable only after years (when the first children develop disease symptoms). The added value of a genetic test is that it could make clear immediately whether the percentage of the mutation is constant from the embryonic stage throughout pregnancy until birth and thereafter. If not, a reliable prediction cannot be made. One should then either re-assess the criteria for embryo selection or refrain from a next application of PGD. Therefore, the importance of the knowledge possibly generated by genetic testing seems beyond question, but several ethical issues need to be scrutinized before one considers testing a newborn. 
If the newborn will be genetically tested, three scenarios are possible. In a first scenario, the postnatal test confirms the genetic diagnosis obtained in the embryonic phase. This suggests that PGD is reliable. In a second scenario, the mutant load has decreased. Although this is favorable for the child, strong doubts will arise regarding the reliability of PGD. In a third scenario, the postnatal test shows a (considerably) higher mutant load. Not only strong doubts will arise regarding the reliability of PGD, this also results in adverse predictive information about the future health of the (possibly asymptomatic) child. Generally speaking, predictive genetic testing of minors is only considered ethically acceptable provided that it is to the medical benefit of the child. In our case, the child undergoing follow-up would probably not medically benefit from testing, although lack of clarity regarding the therapeutic value of early detection exists. Some may argue that lack of cure does not equate lack of treatment (DiMauro et al, 2006). Regular check-ups and some preventive health measures may be beneficial for the child. Others may reply that this is often also done just 'to do something'. Furthermore, although some argue that children may psychosocially benefit from knowledge about their genetic status (Robertson and Savulescu, 2001; Rhodes, 2006), the majority still view that children should not be tested for untreatable late onset disorders (see Bredenoord et al, 2008b). If we assume that follow-up studies are to be classified as non-therapeutic medical research with incompetent participants, what, then, about the ethics?

A research procedure which is not intended directly to benefit the participating child is not necessarily unethical as long as it meets the following requirements (Smith, 1999; WMA, 1964/2008; CIOMS, 2002). Firstly, a preliminary requirement is that the research satisfies the material and procedural requirements for scientifically and ethically sound medical research. This requirement constitutes an additional argument to embed - if feasible - the first applications of PGD for mtDNA mutations in a scientific research protocol. Secondly, 'these individuals (incompetent research subjects) must not be included in a research study that has no likelihood of benefit for them unless it is intended to promote the health of the population represented by the potential subject' (WMA, 2008 art 27). The results of the intended research should mainly benefit the group of patients to which the participant belongs. Whether this requirement can be met depends on the interpretation of 'the population represented'. If this phrase should be understood as referring here (more broadly) to regards all the people carrying mtDNA mutations, then this requirement can be met. After all, the development of reliable preimplantation testing will benefit those carrying mtDNA mutations by giving them reproductive options. Therefore, it promotes their reproductive health. Furthermore, the increased knowledge of mtDNA genetics may eventually contribute to the development of treatment as well, but this 
is highly speculative. If, however, 'the population represented' only includes minors carrying an mtDNA mutation, then it is questionable whether this requirement is met. Thirdly, 'the research cannot instead be performed with competent persons' (WMA, 2008 art 27). This is the case, as the research can only be conducted on children born after PGD and/or PND for an mtDNA disorder. To wait until the child has reached adulthood is not a real alternative. Fourthly, the health and safety risks and the burdens for the research participant should be 'minimal'. Whether the follow-up studies can meet this last requirement depends on different variables in the research design.

A first factor is which tissue needs to be tested. If the use of existing material (e.g., hair, urine and cord blood) could do the job of detecting the mtDNA mutation (Sue et al, 1998), then a muscle biopsy would be unnecessary and unjustified. A second factor is the timing of testing: is it sufficient to collect the material directly after birth, or are parents asked to come back later? Related to this is a third factor, namely the frequency of testing. A fourth factor is whether the test results will be disclosed to the parents.

This latter issue is complex. As the data are easily retraceable to the child in question, anonymity is not possible. In theory, three options therefore remain: (1) to refrain from genetic testing, (2) genetic testing with disclosure to the parents and (3) genetic testing with non-disclosure (that is, withholding the test results from the parents). In practice, the feasibility of withholding test results from the parents is questionable. Therefore, the dilemma is as following. If one refrains from genetic testing, the reliability of PGD cannot be ascertained (or only after years). If one discloses the test results, the predictive genetic information could (psychosocially) harm the child. But, if feasible at all, when one withholds the test results, parents are not able to use the information for possible extension of their family. If it turns out that the PGD procedure did not prevent the birth of a child with a high mutant load, this may guide them and others in future reproductive decisions. If, to the contrary, the child indeed carries no or a low mutant load, they and future users may gain reproductive confidence.

The main ethical assessment to be made, then, is whether disclosure of the knowledge generated by the test results violates the fourth criterion that states that only minimal risk and burden is acceptable. If the child turns out to have a (much) higher mutant load than expected, this may burden the child in a double sense. Firstly, the child grows up with knowledge about its adverse health prospects. How much weight we should give to this knowledge also depends on the expected seriousness of the disease, the age of onset and the chance that symptoms will develop. As only embryos with low mutant load will be transferred, it might be a 
reasonable guess to estimate the chance that the child will develop severe disease symptoms as fairly low. The problem is that this cannot be stated with certainty and that this is precisely the information that the research aims to clarify. A mitigating factor may be that the genotype of the child is already known, albeit in the embryonic phase (and perhaps also in the fetal phase, if a PND has been carried out). The child is born as a result of PGD: it will know about the presence of an mtDNA mutation in the family anyway, also when it is not tested. The second drawback of disclosure may be that a thus far symptom-free child is heading towards a life of hospital visits, check-ups and so on, although no real treatment is available (but the child may have periodic clinical examinations anyway). As we explained above, opinions will differ on how much weight we should ascribe to these drawbacks.

Involving children in this type of research is morally complex. A tension exists between the need to ensure that PGD is safe, effective and reliable and the need to protect the participating children (e.g., Wendler and Varma, 2006). This tension also exists in pediatric drug research. The importance of clinical trials with children versus the acceptability of exposing children to risk has been a topic of increased debate. The question is whether these requirements can be reconciled, or whether the minimal harm standard needs adjustment (e.g., Caldwell, 2004; Wendler and Varma, 2006). In our case, the risks involve psychosocial harm as a result of knowledge about an unexpected high mutant load. We estimate the probability that the child will experience such harm to be small, but real. It is questionable whether these risks satisfy a strict interpretation of the minimal harm standard. If a more flexible interpretation of the minimal harm standard is considered to be acceptable (in the United States, for example, one accepts a minor increase over minimal risk) (Smith, 1999), and if the requirement that this research is necessary to promote the health of the population represented is interpreted flexibly, then these follow-up studies may be ethically justifiable. Even more so because the child will know about the genetic risk in the family anyway.

Although being aware of the many ethical pitfalls, we are inclined to recommend conducting follow-up studies in the context of mtDNA disease. In close deliberation with the parents one could decide whether and how to disclose the test results. Clearly, this complex topic needs further interdisciplinary debate. This debate should regard both the minimal harm standard, the interpretation of the 'population represented' and the acceptability of predictive genetic testing of children.

\subsection{Informed consent}

Obtaining an adequate informed consent is one of the most important moral requirements. What constitutes an 'adequate' informed consent in our discussion? 
What should be the ingredients to be discussed during the counseling process? Table 2 lists the issues that the counselor should bring up for discussion with the prospective parents. Obviously, guiding the couple to make a truly well-informed decision requires both time and professional counseling skills. In the end, much hinges on a transparent communication between prospective parents and the medical team.

\section{Table 2: Information required to support informed consent}

\section{Scientific research}

Ensure that the couple is aware that PGD for mtDNA mutations is not regular practice but experimental (in case PGD is embedded in scientific research).

\section{Residual risks}

Discuss with the couple that PGD may only reduce reproductive risk; they are sufficiently aware that residual health risks may be unavoidable.

Consider to what extent it is possible, desirable and proportional to try to further reduce the health risks.

Moral minimum: avoid a high risk of serious harm

\section{Alternatives}

Discuss possible alternatives such as PND, oocyte donation and adoption. The couple has weighed the pros and cons of these strategies.

\section{Feasibility}

Bring up the possibility (and pros and cons) of oocyte sampling in order to check the feasibility of PGD.

\section{Reliability}

Ensure that the genetic test result is sufficiently representative. This is to be clarified prior to the IVF/PGD procedure will be carried out further. Ensure that the couple is aware that the IVF/PGD procedure will only be continued when the mutation load can be reliably detected and is representative.

\section{Cut-off point}

Discuss the determined cut-off point.

Discuss whether priority should be given to embryos of good morphology or embryos with a lower mutant load.

\section{Number of cycles}

Discuss and determine the maximum number of cycles.

\section{Scientific research after IVF/PGD}

Insist on the possibility to donate affected embryos for scientific research.

Discuss the possibility of regular clinical examination.

Discuss with the couple the importance and pitfalls of conducting a genetic test after birth; if they decide to enroll, they made a decision about whether or not to disclose the test results (and are aware of the possible implications). 


\subsection{Conclusion}

When considering PGD for an mtDNA mutation, PGD may only contribute to a reduction of reproductive risk. Although morally acceptable (Bredenoord et al, $2008 \mathrm{~b}$ ), both the couple and the medical team should carefully look for possibilities to minimize the risks and harms as much as possible. In this chapter, we offered the points to consider when applying PGD for mtDNA mutations in clinical practice. We have discussed the questions that may arise in clinical practice on the basis of three categories of mtDNA mutations, but this framework of course also applies for other heteroplasmic mtDNA mutations. Although far from ideal and with considerable drawbacks, we think that PGD may be a valuable reproductive option for couples at risk of transmitting an mtDNA mutation to their offspring. 


\section{Chapter 5 \\ Reproductive decision-making in the context of mitochondrial DNA disorders: views and experiences of professionals}

Bredenoord AL, Krumeich A, de Vries MC, Dondorp WJ, de Wert G.

Clinical Genetics 2010 Jan;77(1):10-17 


\section{Abstract}

Whereas a scientific and ethical debate about the possible reproductive options for carriers of mitochondrial DNA (mtDNA) mutations is developing, not much information regarding the views and experiences of professionals exists. This chapter explores the attitudes and experiences of professionals involved on a daily basis with their patients' reproductive decision-making in the context of mtDNA disease. Qualitative international multicenter design using in-depth semi-structured interviews with 20 professionals has been utilized. We identified four main themes emerging from the interviews. Firstly, we illustrate the discussion among professionals as to what extent mitochondrial genetics differs from other areas in genetics, both technically and ethically. Secondly, we show the discomfort and doubts of professionals when an mtDNA mutation is involved, because of the uncertainty remaining after testing. Thirdly, we discuss how professionals struggle with the tension between on the one hand the ideal of reproductive autonomy and on the other hand the reality of their professional responsibility and complex clinical decision-making. Fourthly, we delineate the strategies used by professionals in order to make attempts to control uncertainty.

This chapter illustrates the impact on professionals of reproductive decision-making in the context of mtDNA disease. It shows their feelings of discomfort when interpreting and explaining uncertain or ambiguous data and may be perceived as an example of how professionals deal with the inherent limitations in genetic knowledge representing the state of the art. Insight into the experiences of professionals may contribute to a further improvement of reproductive genetic counseling in the context of mtDNA disorders. 


\subsection{Introduction}

With the proliferation of reproductive genetic technology, the options for couples at risk of transmitting a genetic mutation increase. The more technology, the more choice, the more demands are made on the competence of the professional to guide couples in their decision-making process (Mol, 1995).

In this chapter we examine the attitudes and experiences of professionals with their role in reproductive decision-making in the context of mitochondrial DNA (mtDNA) disorders. Women at risk of transmitting an mtDNA mutation increasingly ask for options to avoid the birth of an(other) affected child. However, due to the specific characteristics of mtDNA inheritance, making reliable predictions about the health of future offspring is difficult. Whereas a certain margin of uncertainty always has to be reckoned with when applying prenatal diagnosis (PND) and preimplantation genetic diagnosis (PGD), this counts a fortiori in the context of mtDNA disorders (Bredenoord et al, 2008a). Although morally acceptable under specific conditions, PND and PGD for mtDNA disorders may only reduce reproductive risk and residual uncertainties cannot be ruled out (Bredenoord et al, 2009). Whereas a scientific and scholarly ethical debate about the possible reproductive options for carriers of mtDNA mutations is developing, not much information regarding the experiences of professionals working in mitochondrial genetics exists. What are their concerns, worries and points of view? What do they consider to be the major ethical issues? The purpose of our study was to explore the attitudes and experiences of professionals involved on a daily basis with treating patients with mitochondrial disease and counseling couples with a wish for a child. This chapter aims to illustrate how professionals deal with the uncertainties and ethical peculiarities that are (currently) linked to reproductive decision-making in the context of mtDNA disease.

\subsection{Materials and methods}

The views, experiences and attitudes of professionals were examined by means of a qualitative interview design (Patton, 2002). One-to-one, in-depth, semi-structured interviews $(n=20)$ were conducted with professionals from different background and specialties [see table 3]. It concerned both basic scientists working at the bench and clinicians treating patients and counseling couples with a wish for a child. By selecting respondents of different nationalities and of different profession and background, with diverse views and perspectives, we aimed to collect a range of opinions and experiences as wide as possible (Polit and Beck, 2003).

We selected respondents from four European countries: Italy, France, the United Kingdom and the Netherlands. These countries were co-participants in a EU-funded project on mitochondrial genetics (the MITOCIRCLE-project). Moreover, a broad range of moral and cultural background perspectives is found between and within 
these countries. The respondents were spread over seven (academic) centers. All these centers offer PND for mtDNA disorders, mostly DNA-based tests. Three out of seven centers offer PGD (in general); these three centers would also offer, or already have offered PGD for mtDNA disorders. One center does not offer PGD, because it is legally not allowed.

Table 3 Background of interviewed professionals

\begin{tabular}{|c|c|c|c|}
\hline Number & Department & Profession & Sex \\
\hline P1 & Neurology & Neurologist & M \\
\hline P2 & Pediatrics & Pediatrician & M \\
\hline P3 & Neurology & Neurologist & M \\
\hline P4 & Neurology & Researcher & M \\
\hline P5 & $\begin{array}{l}\text { Reproductive } \\
\text { medicine }\end{array}$ & Gynecologist & $M$ \\
\hline P6 & Clinical genetics & $\begin{array}{l}\text { Clinical } \\
\text { geneticist }\end{array}$ & M \\
\hline P7 & Pediatrics & $\begin{array}{l}\text { Pediatric } \\
\text { neurologist }\end{array}$ & $\mathrm{F}$ \\
\hline P8 & Clinical genetics & Researcher & M \\
\hline P9 & $\begin{array}{l}\text { Reproductive } \\
\text { medicine }\end{array}$ & Gynecologist & M \\
\hline P10 & Pediatrics & $\begin{array}{l}\text { Pediatric } \\
\text { neurologist }\end{array}$ & M \\
\hline P11 & $\begin{array}{l}\text { Reproductive } \\
\text { medicine }\end{array}$ & Gynecologist & M \\
\hline P12 & Pediatrics & $\begin{array}{l}\text { Pediatric } \\
\text { neurologist }\end{array}$ & M \\
\hline $\mathrm{P} 13$ & Pediatrics & Researcher & M \\
\hline P14 & Clinical genetics & Researcher & $F$ \\
\hline P15 & Clinical genetics & Researcher & $\mathrm{F}$ \\
\hline P16 & Clinical genetics & Counselor & $F$ \\
\hline P17 & Clinical genetics & $\begin{array}{l}\text { Clinical } \\
\text { geneticist }\end{array}$ & $\mathrm{F}$ \\
\hline P18 & Neurology & Neurologist & $\mathrm{F}$ \\
\hline P19 & Clinical genetics & Researcher & $\mathrm{F}$ \\
\hline P20 & Pediatrics & $\begin{array}{l}\text { Pediatric } \\
\text { neurologist }\end{array}$ & $\mathrm{F}$ \\
\hline
\end{tabular}


The data were collected between August 2006 and April 2007. All interviews were conducted by $\mathrm{AB}$, except the interviews in Italy. Initial interview topics with open core questions were formulated after examination of the relevant literature. Consistent with standard qualitative research techniques, the interview topics evolved as the interviews progressed through an iterative process to ensure that the questions captured all relevant emerging themes (Britten, 1995). The interviews contained general topics and no close-ended questions (i.e. yes-or-no questions). The interviews lasted between 45 and 75 minutes. All interviews took place on the workplace of the respondent.

All the interviews were audiotaped and transcribed verbatim. Data analysis was based on the constant comparative method (Strauss and Corbin, 1998; Malterud, 2001). This entails the following steps. AB independently coded the full transcripts by identifying and labeling discrete units of texts that referred to one or more concepts relevant to the study purpose. Two researchers checked the codes for consistency (AK, GdW). Through comparison across transcripts, the open codes were developed into higher order themes to provide a framework for coding subsequent transcripts. When no new thematic content was found in the interviews, subject enrolment was stopped. This process, called thematic saturation, is a well-described qualitative method to avoid unnecessarily large and repetitive data sets (Guest et al, 2006; Denzin and Lincoln, 2005). Finally, representative quotations were chosen to demonstrate the themes identified; these are noted in italics in the results section by participant number.

\subsection{Results}

We identified four main themes emerging from the interviews. Firstly, we illustrate the discussion among professionals as to what extent mitochondrial genetics differs from other areas in genetics, both technically and ethically. Secondly, we show the discomfort and doubts of professionals when an mtDNA mutation seems involved, because of the uncertainty that often remains after testing. Thirdly, we discuss how professionals struggle with the tension between on the one hand the ideal of reproductive autonomy and on the other hand the reality of their professional responsibility and complex clinical decision-making. Fourthly, we delineate the strategies used by professionals in order to make attempts to control the uncertainty.

\section{Theme 1. Is mitochondrial genetics different?}

"My first question is, really, from first principles, is the issue of mitochondrial disease ethically different to other diseases?" (P1) 
This question is evoked by most of the professionals. Most of them struggle with the question to what extent mitochondrial genetics resembles other areas in genetics. In other words, how peculiar is mitochondrial genetics? For some, the complexity is not exclusive for (reproductive) genetic testing in mitochondrial genetics, as no genetic test is without risks and uncertainties. From this perspective, uncertainty is an inherent part of genetic medicine and mitochondrial genetics should not be given a status aparte.

"I would compare mtDNA with all the testing activities in the field of susceptibility testing, where, at the end, the contribution of the test results explain only $20 \%$ or $30 \%$ or $50 \%$ of the likelihood that the disease will develop or not. (...) We should not make it an exceptional case, although the uncertainty regarding the interpretation of test results is indeed somehow different" (P17)

Most professionals, however, notify that the challenges of reproductive genetic testing and counseling are intensified in the context of mtDNA disease. Professionals frequently brought up the complexity of interpreting mtDNA heteroplasmy and the difficulty of excluding that an mtDNA mutation is involved.

"Actually, the whole of genetic medicine is a continuum, you know, the blurriness of mitochondrial diseases is not, in principle, any different from the blurriness in Huntington's disease actually, because there are late onset mild forms, which just involve one little twitchiness, and there are young onset severe forms which involve dementia before your twenties. And actually, the genetic test doesn't separate those two, you can't separate them. (...) But in Huntington's, at least you have got one situation which is cast in tables and stone, that is, if you haven't inherited the change, you won't be affected. You can't say that with mitochondrial disease" (P1)

"The peculiarity is that you are never completely confident that an mtDNA mutation has been excluded. And the other compound of uncertainty is of course the level of heteroplasmy (...) So when you are dealing with heteroplasmy, the ethical problem is again that it is a quantitative genetics, it's not Mendelian 'yes or no'” (P12) 


\title{
Theme 2. Professional reactions when an mtDNA mutation is suspected
}

The uncertainty inherently linked to mitochondrial genetics is discomforting for the professionals involved, because they prefer to communicate certainties.

\begin{abstract}
"Also the physicians are very anxious when mtDNA mutations are involved. In those cases there are heated discussions in the staff. Physicians want certainty, which is exactly the problem" (P16)
\end{abstract}

"As a physician I would like to pass judgments as hard as a rock. Not ambiguous judgments. They [the couple] take a decision that is based on my opinion, which is not robustly enough. I find that regrettable" (P7)

Professionals indicate that these are difficult situations, not only for the couple but for the professional as well.

"Until you make sure that no mtDNA mutation is involved, until that time you cannot say a word. So this is the most risky situation for the geneticist. To advise families. You can be face to face with a lady who is carrier, and you don't know. She has no symptoms, nothing" (P12)

Some perceive the situation to be so uncertain, that they are inclined to not offering specific type of tests anymore:

"Our approach [with offering PND for mtDNA disorders] is so cautious that we have almost no experience" (P20)

"So, more and more often we do not propose enzyme-based testing for these mutations anymore. It's less and less, because we are not very confident of the results" (P14)

Others propose to not offering testing for specific types of mutations. Regarding some mutations, professionals are more insecure than with other mutations. When for example discussing PND and PGD for the m.3243A>G MELAS (mitochondrial myopathy, encephalopathy and stroke-like episodes) mutation professionals are more reserved than with other mutations:

"I think it is on the edge [PND and PGD for MELAS] and it is questionable whether we should pass that line. It would keep me awake" (P8) 
Many professionals thus show cautiousness when an mtDNA mutation is involved. As a consequence, some professionals exclude particular types of testing, or they exclude certain mtDNA mutations. What, then, once they have decided to go ahead with a specific couple? How do they view the counseling process?

\section{Theme 3. Tension between the ideal of reproductive autonomy and the reality of clinical practice}

For most professionals, reproductive decision-making is 'teamwork', in which the clinician, the researchers and the couple together form a team. However, to be able to make informed decisions, the couple should be provided with information on at least a part of the complexities and uncertainties of mitochondrial genetics. They should also, according to professionals, understand the remaining uncertainties. Although all professionals identify reproductive autonomy as a core value, they do not agree about whether couples are capable of making autonomous decisions when the information provided is so complex and ambiguous. Some are quite positive about the possibilities to explain the ins and outs:

"I use images, symbols and examples and they understand it very well. Believe me, even the very simple, not-educated are not stupid" (P12)

"For me, there is absolute no problem in using tests for which we have difficulties or which have limits, as far as you inform patients on these limits if you have doubts. So this is a general approach in medicine: we have a possible treatment for you, we don't know yet if it will work or not, nor whether it may have adverse effects. But if you inform the patient, in the end the patient, after balancing, can make her own decision (...) It's [informing couples] a matter really of time. I know that for complicated decisions you need at least 45 minutes or one hour with a couple or a patient. And most physicians cannot spend that much time with patients. But if you have the resources to do that, it works" (P17)

Others, however, question whether the nuances of mitochondrial genetics can be explained to couples:

"One of the difficulties, of the ethical bits that actually no one challenges is the fact that actually to understand this [information regarding $m t D N A$ ], you have got to have a certain level of intellectual capacity. And this is an awful thing to say, but the truth is that only a proportion of the population can handle this information and understand it, I believe. (...) If a physician 
cannot make a decision, based on the evidence, how in earth the others?" (P1)

"The clinical phenotype varies and people visit us to have a healthy child, so you really have to counsel them well in order to explain everything. The hospital setting implies that people are inclined to think that everything will be all right and that their child will be healthy after all" (P8)

\section{Theme 4. Strategies to control the uncertainties}

Because of the uncertainties in predicting the phenotype and the complexities in explaining these uncertainties, some professionals do not offer specific types of genetic testing or they exclude testing for some mutations. Others, however, are more positive towards offering tests, but this is accompanied with the introduction of several mechanisms to control and reduce the uncertainties. Most professionals are looking for 'steady ground', for control.

Several strategies are used to try to control the uncertainty. First of all, many professionals introduce some kind of quality requirement: they emphasize that only very experienced and well-equipped professionals should offer testing in the context of mtDNA disease. Furthermore, they also stress that the centers that currently offer genetic testing for mtDNA disease are highly qualified and conduct cutting edge research.

"Doing the biochemistry is really tricky. So you don't want people doing it who don't have a lot of experience" (P3)

"Also the patient organizations regard the European centers at cutting edge in mitochondrial genetics" (P12)

Secondly, many professionals emphasize the importance of international cooperation in order to improve scientific knowledge.

"We have got to work together as a [mitochondrial] community on this, because each individual centre still has a very limited amount of information" (P3)

"In order to generate more knowledge with regard to mtDNA we should establish big databases, as well as multidisciplinary and international research, like the MITOCIRCLE project. The most important is that we share and exchange our experience" (P8) 
"What I think is useful is that we have different approaches in different groups, to have different answers. Not all the labs can do everything so it is good to specialize and we will see after two or three years of research what is the best way to go" (P14)

A third reaction to gain control contains the efforts of professionals to try to calculate the recurrence risks as precisely as possible. Professionals seem to feel uncomfortable that they cannot fulfill what they regard as their main job, namely providing expert knowledge on the basis of data that are precise and well understood, also in terms of clinical consequences. They are very anxious to find out the precise risks and to communicate these to their patients.

\begin{abstract}
"What interests me the most really is just how difficult it is to explain recurrence risks to patients. It is easy mathematically, but really what does that mean for a person, you know, when you are faced with a decision whether or not you should go ahead with a pregnancy. Now that's just separate to include a 25,50 or $75 \%$ risk in rare circumstances. What's happening when you start breaking that up all in between? What on earth does that mean? $18 \%$ or $33 \%$, what does that mean? (...) Sometimes, as a kind of opposite argument to what I just presented to you about the uncertainty of the data, I sometimes think maybe we are trying to be too good, too accurate" (P1)
\end{abstract}

\title{
5.4. Discussion
}

Our results show that all professionals feel that the inherent challenges of reproductive genetic testing and counseling are intensified in the context of mtDNA disease (theme 1). Even for the few professionals that did not explicitly state that they perceive mitochondrial genetics to be different, one may still infer this from their reactions to offering PND or PGD (theme 2). The uncertainties linked to mitochondrial genetics seem to confuse professionals. They tend to regard these uncertainties as conflicting with their task of providing accurate information when counseling couples. Many feel uncomfortable by not being able to communicate definite and unambiguous information. Mitochondrial genetics is currently characterized by two types of uncertainty: uncertainty in predicting the recurrence risk of a couple and uncertainty in predicting whether a child will develop clinical disease symptoms, and if so, how serious these will be. So, when an mtDNA mutation is involved, it is not business as usual.

This has consequences for the framework in which professionals usually assist and counsel their patients. Genetic counseling is usually perceived as a process aimed at 
enabling couples to take meaningful decisions. Information is not given as an end in itself, but as a means of helping people to reach a personally satisfactory decision (Shiloh et al, 2006a). Similarly, most professionals in our study view reproductive decision-making as both a process and as 'teamwork', in which the clinician, the researchers and the couple together form a team. However, in order to be able to make informed decisions, the couple should be informed about at least a part of the complexities and uncertainties of mitochondrial genetics. All professionals identify reproductive autonomy as a core value, but they disagree as to whether couples facing the risk of transmitting an mtDNA mutation are capable to make informed, autonomous decisions with regard to the reproductive options open to them (theme 3). Mitochondrial genetics may therefore be perceived as a test case for reproductive autonomy. To answer the ethical question whether and under which conditions PND or PGD in the context of mtDNA disease is acceptable falls outside the scope of this paper, but is discussed elsewhere (Bredenoord et al 2008b; Bredenoord et al, 2009). It is at the least interesting that in our interviews different professionals draw different conclusions. Whereas a part of the professionals hold the opinion that reproductive autonomy is not endangered, others doubt whether the nuances of mitochondrial genetics can still be explained.

Because of the uncertainties in predicting the phenotype and the difficulties in explaining the ins and outs, some professionals conclude that either specific reproductive genetic tests should currently not be offered or that these tests should not be applied to all mtDNA mutations. Others draw the conclusion that one may go ahead, but only in accompaniment of several mechanisms to control and reduce the uncertainties (theme 4). So, these tests may be offered, but only by professionals and laboratories with the highest qualifications and only in the context of the best possible international collaboration and research programs. Furthermore, these professionals try calculating and explaining the recurrence risks as adequately as possible. They hold the view that the best possible way of dealing with uncertainties is to calculate the risks as precisely as possible.

Although knowing the magnitude of any remaining risks is necessary for determining whether testing can justifiably be offered, and although it is interesting from a scientific point of view, more precise calculations will not always provide the couples involved with a better basis for decision-making. Even apart from the fact that - as studies indicate - patients have a poor understanding of quantitative risk information (Lloyd, 2001), it could be questioned whether it will be helpful for couples to know whether the risk that their child will be clinically affected is $33 \%$ or $39 \%$ (with the qualification that even mtDNA specialists are currently unable to predict such a risk level). From this perspective, the last quoted citation is striking: perhaps 
professionals are trying to be too accurate. Indeed, research suggests that counselees are not very interested in more precise risk information. They long for information providing them with a high degree of certainty about expected outcomes (van Zuuren et al, 1997; Shiloh et al, 2006b). Whereas genetic information is normally already replete with uncertainties, this is even more so in the context of mitochondrial disease.

Although we can imagine that some professionals conclude that reproductive genetic testing for couples affected by an mtDNA mutation is too challenging, we think that this should be decided on a case-by-case basis. After all, some reproductive options may considerably bring down the transmission risk. Although challenging, this still can be a meaningful option for couples who are willing and able to deal with the uncertainties involved. In our view, it belongs to the responsibility of professionals working in this field to try to help affected couples to make a well-informed decision, even if this cannot always consist in providing them with precise data about remaining risks. The uncertainties inherently linked to genetic information in general and to mitochondrial genetics specifically could be the starting point of the counseling process. Furthermore, studies show that counselees find information about the consequences of having an affected child and of alternative reproductive options very helpful (Shiloh et al, 2006a;b). So, professionals could pay attention to the consequences of living with a child with a mtDNA disorder, the range of possible clinical manifestations, the expected quality of life and the possibilities and limitations for the child (and its siblings). As predicting whether a child will be clinically affected (and if so, how serious) may remain difficult, it could be helpful to discuss several scenarios with the couple (i.e. the worse case scenario as well as a more favorable outcome) (Abramsky, 2003). Here, the role of the counselor is to help people to see what all the possible consequences of the different reproductive options are and how these relate to their circumstances and values (Marteau and Dormandy, 2001). This implies that the counselor and couple should discuss the uncertainties and moral dilemmas involved, the different reproductive strategies for carriers of mtDNA mutations and their pros and cons.

Obviously, this type of counseling is time-consuming. We recommend to seriously building in extra time to provide people with the appropriate information. This is a sine qua non for guaranteeing that reproductive autonomy is more than just a catchy term and for assuring that people make well-informed decisions. Furthermore, to concentrate testing and counseling for mtDNA disease in some specialized centers guarantees that researchers and counselors are capable of developing more experience in mtDNA based counseling. 


\subsection{Conclusion}

This paper illustrates the impact of reproductive counseling and decision-making in the context of mtDNA disease on professionals. Mitochondrial genetics is currently characterized by two types of uncertainty: uncertainty in predicting the recurrence risk of a couple and uncertainty in predicting whether a child will develop clinical disease symptoms, and if so how serious. This study shows that professionals feel discomfort when handling, interpreting, and explaining uncertain or ambiguous data. As some professionals doubt whether the nuances of mitochondrial genetics can still be explained, mitochondrial genetics may be perceived as a test case for reproductive autonomy. Furthermore, this study may be perceived as an example of how professionals deal with the inherent limitations in genetic knowledge representing the state of the art. After all, novel developments in medical genetics will continuously confront professionals with ambiguous information. Part of the uncertainty in mitochondrial genetics may be caused by insufficient knowledge and may therefore be reduced by future research. However, part of the uncertainty may be inherently linked to mtDNA genetics. In that case, reproductive counseling and decision-making will always remain complex in the context of mtDNA disease. 



\section{Chapter 6}

Ooplasmic and nuclear transfer to prevent mitochondrial DNA disorders: conceptual and normative issues

Bredenoord AL, Pennings G, de Wert G.

Human Reproduction Update 2008 Nov-Dec;14(6):669-678 


\section{Abstract}

Mitochondrial DNA (mtDNA) disorders are an important cause of human diseases. In view of the limitations of prenatal diagnosis (PND) and preimplantation genetic diagnoses (PGD), alternatives such as ooplasmic transfer (OT) and nuclear transfer (NT) have been proposed to prevent the transmission of mtDNA mutations. Both OT and NT are radical in the sense that they do not entail genetic selection, but genetic intervention to correct the genetic cause of the disease. After interviews with experts in the field, the relevant literature was searched and analyzed. Bioethical issues were divided into conceptual and normative points.

OT is the transfer of normal mitochondria to a carrier's oocyte containing mutant mtDNA. In case of NT, a donated oocyte is enucleated and replaced with the nuclear DNA from a woman carrying an mtDNA mutation. NT can be performed both before and after in vitro fertilization, respectively, with the nucleus of an unfertilized oocyte, with the pronuclei of the zygote, or with the nucleus of a blastomere of an embryo. Conceptual questions regard whether these techniques amount to germ-line modification and human cloning. Normative questions concern, among others, the significance of intervening in the mtDNA, the implications of having 'three genetic parents', the ethics of oocyte donation and the health and safety risks for children conceived as a result of one of these techniques. Further interdisciplinary debate and research is needed to determine whether a clinical application of OT and NT can be morally justified, and if so, under which conditions. 


\subsection{Introduction}

The Human Fertilization and Embryology Act (1990), one of the world's first laws enacted to regulate developments in assisted reproductive technology and embryo research, is currently under review. In December 2007, the UK House of Lords discussed a special amendment of the law, containing a proposal to permit pronuclear transfer to prevent mitochondrial DNA (mtDNA) disorders. The heated political debate focused on the acceptability of germ-line modification, the fear of reproductive cloning and the significance of the mtDNA. Earlier, in 2005, the Human Fertilization and Embryology Authority (HFEA) granted a license to determine the feasibility of pronuclear transfer (Brown et al, 2006). This license also evoked public and political attention (e.g. Randerson, 2004). Pronuclear transfer is not the only strategy that has been proposed as a possible preventive option for women at risk of transmitting an mtDNA mutation to their offspring.

Mitochondrial disorders can both arise from nuclear gene mutations and from defects in the mtDNA. MtDNA mutations are an important cause of human diseases, although the precise prevalence is difficult to estimate (Taylor and Turnbull, 2005). Mitochondrial disorders usually are severe disorders, involving defects in energy production. Although mtDNA disease may present 'with any symptom in any organ at any age' (Munnich et al, 1996; Haas et al, 2007), often the most energy demanding tissues such as the central nervous system, heart and skeletal muscles, liver and kidneys are affected. Because there is no curative treatment (Taylor and Turnbull, 2005; Chinnery et al, 2006), the prevention of the transmission of mtDNA disorders is considered to be of key importance. Although prenatal diagnosis (PND) and preimplantation genetic diagnosis (PGD) are morally acceptable in specific circumstances, the application of both techniques for mtDNA disorders raises complex ethical questions (Bredenoord et al, 2008a,b). In view of the limitations of PND and PGD, the scientific community is searching for 'radical' alternatives, including ooplasmic transfer (OT) and (pro)nuclear transfer (NT). These approaches are radical in the sense that they do not entail genetic selection, but genetic intervention (or modification) to correct the genetic cause of the disease. These techniques aim to avoid mitochondrial disorders by intervening at the very beginning.

As preclinical experiments are currently performed and the clinical application of pronuclear transfer may be seriously considered in the near future, this chapter proactively identifies the ethical issues involved. After interviews with experts in the field, we searched and analyzed the relevant literature (using a so-called snowball method). Bioethical issues were divided in conceptual and normative aspects of OT and NT. Our aim is to clarify these complex conceptual and normative aspects and to 
scrutinize both the ethical questions surrounding the development of those techniques in the laboratory as well as their possible clinical application. The main part of this chapter will focus on NT (instead of OT) for three reasons: first, NT seems more promising, secondly, recently doubts have risen regarding the value of OT in patients with mtDNA disease (Brown et al, 2006) and finally, the conceptual and normative questions for both techniques largely overlap.

\subsection{Ooplasmic transfer}

In the literature, both the terms ooplasmic transfer and 'cytoplasmic' transfer are being used. In this paper, we use the more precise term ooplasmic transfer. This is the transfer of donor ooplasm with normal mitochondria to an oocyte containing mutant mtDNA. It has been introduced as an assisted reproductive technique for women who experienced repeated embryonic development failure. As defective ooplasm may cause infertility, normal mitochondria from a donor oocyte are transferred into the patient's oocytes (Cohen et al, 1997, 1998; Huang et al, 1999; Lanzendorf et al, 1999; Barritt et al, 2001a,b; Krey et al, 2001; de Wert, 2003; Jacobs et al, 2006). This may provide the mitochondrial 'boost' necessary for embryonic development to prevent IVF failures (Van Blerkom et al, 1998). OT would also dilute or reduce the effect of mtDNA defects (Kagawa and Hayashi, 1997). However, the suitability and feasibility of OT for preventing mtDNA disease has been doubted for two reasons. First, in the initial applications in women experiencing repeated embryonic development failure, a relatively high number of chromosomal abnormalities and birth defects after OT have been reported (Jacobs et al, 2006; Brown et al, 2006). Secondly, in those applications only small amounts (10-15\%) of donor ooplasm were transferred to the recipient's oocyte. To prevent the transmission of mtDNA disease, a larger amount (up to $50 \%$ ) of donor ooplasm is needed. It is seriously questioned whether it is possible to introduce such an amount of ooplasm into the oocyte. The consequence is that the relative proportion of mutated to wildtype mtDNA is unlikely to change enough to prevent clinical disease (Thorburn and Dahl, 2001; Jacobs et al, 2006; Taylor and Turnbull, 2005; Brown et al, 2006; Fulka et al, 2007; Gardner et al, 2007).

\subsection{Nuclear transfer}

Although the technique was proposed earlier, it was only in 1999 that Roberts announced that 'a new human reproductive option now has the potential to eliminate the possibility of recurrence of mitochondrial disease in affected families' (Rubenstein et al, 1995; Roberts, 1999:265). In case of NT, a donated oocyte is enucleated and replaced with the nuclear DNA from a woman carrying an mtDNA mutation. As the woman's female relatives could be carriers as well, the donated oocyte would 
preferably be from the paternal side of the family or from an unrelated donor. The nuclear transplantation can be performed both before and after in vitro fertilization (IVF), respectively, with the nucleus of an unfertilized oocyte, with the pronuclei of the zygote (biopsied during the fertilization process), or with the nucleus of a blastomere of a fertilized oocyte, i.e. an embryo (de Wert, 2000; Brown et al, 2006). The current studies in the UK regard the feasibility of this second variant of NT, also called pronuclear transfer (Brown et al, 2006; Gardner et al, 2007).

\subsection{Conceptual issues}

\subsubsection{Is OT and/or NT a type of germ-line modification?}

A first conceptual issue concerns whether OT and/or NT should be classified as forms of germ-line modification. This question can be answered both affirmatively and negatively, depending on what is determined as the defining characteristic of germline modification. In general, a modification of the germ-line means that new genetic material is introduced into the gametes (or early embryo). This genetic modification is not only passed on to the child, but also to subsequent generations. In this sense, both OT and NT can be perceived as forms of germ-line modification. The mtDNA of the oocyte of the affected mother is supplemented with (OT) or exchanged for (NT) mtDNA of a donor oocyte and therefore irreversibly changed (Rubenstein et al, 1995; de Wert, 1999; Health Council of the Netherlands, 2001; Thorburn et al, 2001), although it can be questioned whether the small amount of mitochondria introduced in case of OT will pass to the next generation.

Initially, germ-line modification was synonymous with changes in the nuclear DNA. When the definition of germ-line modification is restricted to modification of the cell 'nucleus', then OT and NT would not amount to germ-line modification. After all, the cell nucleus remains intact. Both OT and NT to prevent mtDNA disease can be classified as a therapeutic germ-line intervention (or modification), whereby only the cell membrane is penetrated but the nuclear membrane is not (Wivel and Walters, 1993). They encompass a type of germ-line modification in which only the mtDNA is changed, which is extra-nuclear DNA.

Clearly, this conceptual issue is highly relevant for the normative analysis of OT and NT. After all, human germ-line modification is ethically much more controversial than somatic genetic modification. It is prohibited by international organs, such as the Council of Europe (Council of Europe, 1997 Art 13: 'an intervention seeking to modify the human genome may only be undertaken for preventive, diagnostic or therapeutic purposes and only if its aim is not to introduce any modification in the genome of any descendants'). Some countries, though, legally make a distinction between germ-line modification of the nuclear DNA and germ-line modification of the mtDNA. The Dutch 
Embryo Act (2002), for example, leaves room for modifying the mtDNA, while modification of the nuclear DNA is prohibited. Also the HFE Act 2007 review proposes to prohibit modifications of both the nuclear and the mtDNA "with an exception' for modifications of the mtDNA that are designed to prevent the transmission of serious mitochondrial disease.

The question is whether the strict dichotomy that is made between nuclear DNA and mtDNA is tenable. Much, after all, is unknown about nucleo-mitochondrial interaction (St John et al, 2004; Poulton et al, 2006; Bowles et al, 2007). Modifications in the mtDNA may also influence nuclear gene expression. What does it mean to alter the match between nuclear and mitochondrial complements (Bonnicksen, 1998)? The ethical assessment of germ-line modification (in the context of mtDNA disorders) should therefore also reflect on the significance of the distinction between nuclear DNA and mtDNA.

\subsubsection{Is nuclear transfer a type of human cloning?}

The second conceptual issue, relevant for NT only, is whether it amounts to human cloning. This question also can be answered both affirmatively and negatively, depending on what is determined as the defining characteristic of cloning and on whether fertilized or unfertilized oocytes are used (de Wert, 2000; Jacobs et al, 2006). Usually, cloning is defined as the production of a genetic copy, a genetically identical organism or individual ('genetic duplication'). Some may deny that NT is a form of cloning because this application is not aimed at the conception of genetically identical individuals but at the prevention of severe genetic disorders. But what is, or should be, the essential or defining characteristic of cloning? Is it the aim, the technique and/or the results?

As there is much confusion about the terms used in the debate on human cloning (President's Council on Bioethics, 2002), a further clarification of the concept is needed for the moral evaluation. Several distinctions and definitions have been proposed (de Wert, 2000). A first distinction is between embryo cloning (when the original cell is derived from an embryo) and adult cloning (when the original cell is derived from an adult). A second distinction is between reproductive cloning and nonreproductive cloning. Whereas reproductive cloning aims at the conception of a child, non-reproductive cloning aims primarily at obtaining stem cell lines for research. It is therefore also called 'therapeutic cloning'. This latter distinction has, however, been criticized from two different sides. A first criticism comes from those who object to the distinction between reproductive and non-reproductive cloning, because all cloning would be reproductive. After all, so they argue, all human cloning entails the production of a cloned human embryo. This group prefers to use instead the 
terminology 'cloning to produce children' and 'cloning for biomedical research' (President's Council on Bioethics, 2002). A second criticism comes from those who argue that cloning should exclusively be reserved for 'cloning to produce children'. Instead of cloning for research, they use 'somatic cell nuclear transfer' (SCNT) or 'nuclear transplantation'. This is mainly inspired by the wish to avoid the negative connotations that stick to the term cloning. This distinction, in turn, is criticized because it only describes the technique but fails to convey the nature of the deed itself (President's Council on Bioethics, 2002).

Clearly, no consensus exists regarding the adequate terminology. Taking into account the preceding discussion, we use in this paper the following classification of human cloning:

1. Research cloning: all applications where cloning is used for research.

2. Reproductive cloning: all applications where cloning results in a new human being/individual.

a. Reproductive embryo cloning: when the original cell(s) is derived from an embryo.

b. Reproductive adult cloning: when the original cell(s) is derived from an adult human being.

Particularly 'reproductive' cloning is highly controversial and prohibited both by international organizations and by many national laws. The United Nations Declaration on Human Cloning (1998) calls upon member states to prohibit all forms of human cloning and the Council of Europe's Additional Protocol on the Prohibition of Cloning Human Beings forbids 'any intervention seeking to create a human being genetically identical to another human being, whether living or dead' (Council of Europe, 1998).

Does NT to prevent mtDNA disease amount to human cloning, and if so, to what category? It is important to have a look at the technique of NT. After all, the nuclear transplantation can be performed with:

1. The nucleus of an 'unfertilized' oocyte (NT type 1).

2. The pronuclei of a 'half fertilized' oocyte (the zygote; i.e. the sperm has penetrated the oocyte but no fusion or syngamy has yet occurred) (NT type 2).

3. The nucleus of a 'blastomere' of an embryo (NT type 3).

The first and second type, where either the nucleus of an unfertilized oocyte or the pronuclei of a zygote are transplanted, do not amount to human cloning, because no 
genetically identical twins are created. No duplication occurs. The second and third strategies differ only minimally in time. The period where the oocyte is 'partly fertilized', but the genetic material is not yet fused (the so-called presyngamy stage) constitutes only a thin, but significant line. The third type, where the nucleus of a blastomere is transplanted, does constitute human cloning, more specifically reproductive embryo cloning (de Wert, 2000). The fact that the new embryo has different mtDNA and thus is not entirely identical to the original embryo does not change this conclusion. After all, cloning is usually defined as sharing identical nuclear DNA (Cohen and Tomkin, 1994; Council of Europe, 1998; Health Council of the Netherlands, 2001). By the way, NT type 3 does not necessarily result in the birth of genetic identical individuals. This depends on the number of embryos transferred to a womb and subsequently on the number of children thus created (this will be discussed below).

The two conceptual questions regarding germ-line modification and cloning show that definitions and the choice of a specific type of technique raise different moral questions. Germ-line modification is at least as controversial as human cloning. Questions as whether modifications in the mtDNA should be the exception to the ban on germ-line modification (such as in Dutch law) or whether 'mitochondrial disease pose[s] a unique ethical argument for human cloning' (Roberts, 1999:265), demonstrate that the prevention of mtDNA disease by means of these technologies raises puzzling issues. We will now turn to the normative issues surrounding both OT and NT.

\subsection{Normative issues}

\subsubsection{The moral value of embryos}

Embryo research is required in the preclinical phase for the development of nearly every new reproductive technique. This is the case for OT, and this also applies to the development of NT. The aim of the preclinical studies is to check the safety and feasibility of NT and to make a preclinical risk assessment (which is discussed below). The use of embryos for research is unacceptable for those ascribing high or absolute moral status to embryos. According to this position, all wastage of embryos is dismissed, the discarding of supernumerary embryos after IVF included. For those ascribing low or no independent moral status to embryos, embryo research is justified if 'for good reasons' and on the condition that the providers of the gametes gave their consent. In many experiments embryos left over after IVF can be used, but for the development of NT it may be necessary to create embryos especially for research as well. Creating embryos for research is rather controversial. It is also suggested that creating human-animal cybrids may provide the opportunity to learn more about the 
biology of mitochondria (St John and Lovell-Badge, 2007). Also this practice is controversial.

Although embryos are needed in the preclinical phase, a moral advantage of NT over other strategies, such as PGD and PND, could be that eventually no embryos or fetuses are lost-it may, in the end, be 'embryo-saving'. After all, the whole rationale behind PGD is embryo selection. The embryos not selected for transfer will be discarded. In case of PND, an adverse test result may lead to a termination of pregnancy. NT type 1 on the contrary only uses oocytes and NT type 2 only sperm and oocytes. NT type 3 would in theory only use one embryo (although in practice probably more embryos would be created, mainly because of the inefficiency of the technique). Whether it is acceptable to use embryos as a means to save embryos and fetuses in the long run depends, again, upon one's view of the status of the embryo.

In their experiments on NT type 2 (pronuclear transfer), the Newcastle researchers use abnormally fertilized human embryos (Gardner et al, 2007). More precisely, they use tripronuclear zygotes leftover after IVF. Tripronuclear embryos clearly are not viable. This makes (provided one ascribes low status to embryos) their use ethically less problematic: they would not have been eligible for transfer, and thus would be discarded anyway. Although the use of tripronuclear embryos may not be that problematic, using such embryos does lead to a puzzling conceptual issue: what exactly constitutes an embryo? The Dutch Embryo Act (2002) defines an embryo as a cell or cluster of cells with the potential to develop into a human being (art 1c). Clearly, according to Dutch law, non-viable embryos such as tripronuclear zygotes are not embryos. In theory, researchers thus can use them for whatever reason they like and without ethical review. This seems counterintuitive and a gap in the law (de Wert, 2001; Dondorp and de Wert, 2005; Olsthoorn-Heim et al, 2006). Likewise, when the embryo is defined as a fertilized oocyte, then the resulting 'organism' of all techniques in which other 'modes of production' are used (such as somatic cell nuclear transfer, i.e. cloning) would, strictly speaking, not be embryos either. That may lead to counterintuitive conclusions as well. To the extent that this organism would be capable of growing into a human being it would have the same claim to protection as do embryos resulting from fertilization (Dondorp and de Wert, 2007). The question, therefore, that urges on further reflection is: what makes something a human embryo (e.g. Devolder, 2006)?

\subsubsection{Health and safety risks}

The introduction of OT in the clinic has been a controversial process. In the initial applications of OT for women experiencing repeated embryonic development failure, 
a relatively high number of chromosomal abnormalities and birth defects has been reported (Jacobs et al, 2006; Brown et al, 2006), but it is unclear whether this is related to the technique of OT (Barritt et al, 2001b). Commentators criticized the premature introduction of OT in the clinic. They pointed at the absence of basic preclinical research and suitable experimental controls. They furthermore argued that, when applying 'pioneering' methods, the safety and efficacy should be evaluated in animal models first, followed by a public discussion and ethical review (de Wert, 1999; Parens and Juengst, 2001; Hawes et al, 2002). Subsequently, the Food and Drug Administration declared in 2001 that OT and related protocols are subject to formal review and approval (Zoon, 2001). It was emphasized that before widespread application is considered, conducting animal research and (pre)clinical trials is important to address the unanswered questions regarding both the efficacy and safety of the technique (Templeton, 2002; Brenner, 2004). It is, by the way, questioned whether obtaining such review and approval will be feasible: 'the controversial nature of such human gamete manipulation has resulted in a premature de facto ban on the clinical trials necessary to address these issues' (Malter, 2002:119).

Preclinical scientific research to assess the feasibility and safety of NT is currently being performed with (tripronuclear) embryos left over after IVF (see above). When NT proves to be successful and effective in preclinical feasibility studies, the step to the clinic may one day be considered. Some argue that huge problems need to be overcome before entering into the clinical phase may be considered (Poulton et al, 2006). Regarding both OT and NT, two possible health risks are emphasized.

First, it is unknown whether a mixture of mtDNA from two different origins is safe (Thorburn et al, 2001). In the case of NT, small amounts of affected mtDNA may come along with the nucleus or pronuclei, resulting in mtDNA heteroplasmy (Spikings et al, 2006; Bowles et al, 2008). Obviously, it is important to know the amount of mtDNA that may come along. If the amount of mutant mtDNA would exceed the threshold to disease expression, then NT may overreach its goal. In the case of OT, there is an inherent mixture of mtDNA. Evidence of mtDNA heteroplasmy was present in several children born after OT (Brenner et al, 2000; Barritt et al, 2001a,c). Although some bring to the fore that there is no reason to consider the minimal proportion of detected donor mitochondria observed in the offspring as harmful (Barritt et al, 2001a), the effects on children are unknown.

Second, much is still unknown about epigenetic factors such as nucleo-mitochondrial interaction (St John et al, 2004; Poulton et al, 2006; Bowles et al, 2007). What will happen in the case of NT when the mtDNA is replaced? Some argue that scientific knowledge is insufficient to assess the risk/benefit ratio of NT (Szebik, 1999). 
Although determining acceptable health risks to mother and future child is a tricky enterprise for all new reproductive technologies, a complicating factor is that NT generates irreversible changes. Whatever effect is produced, it will be passed on in the germ-line to successive generations. Sufficient knowledge about the possible risks is therefore even more important. Article $5 \mathrm{a}$ of the United Nations Universal Declaration on the Human Genome and Human Rights (1997) states that 'research, treatment or diagnosis affecting an individual's genome shall be undertaken only after rigorous and prior assessment of the potential risks and benefits pertaining thereto'. But how to put this into practice? The introduction of IVF and ICSI showed that the safety of assisted reproductive technologies can only be proven after many years. How to adequately assess the potential risks and benefits in order to govern new reproductive technology? A principle often referred to is the Precautionary Principle. Although this principle comes in many versions, the strong version counsels that we should refrain from acting until safety is established through clear evidence: 'better safe than sorry' (Sunstein, 2002-2003) (or the Hippocratic prescription: in dubio abstine). Where some argue that taking precautions makes good science (e.g. Howard and Saunders, 1999), others argue that it stifles discovery or paralyzes scientific and technical progress (Harris and Holm, 1999; Sunstein, 2002-3). To govern the introduction of new reproductive technology one could also adhere to a 'proof first' approach, placing the burdens on the regulator to demonstrate a high risk of serious harm (Harris, 2007). Intermediate approaches may be conceivable as well.

Most if not all will agree that a decent minimum of safeguards for the health of the children is morally required-or perhaps even the first consideration (Peters, 2004; President's Council on Bioethics, 2004). Applying new technique without those safeguards resembles the premature introduction of new drugs without proper research (Pennings et al, 2007b). The question of course is when enough safeguards are made to justifiably introduce experimental reproductive and genetic technologies in general, and OT/NT in particular, in the clinic. Furthermore, a related question to be addressed is whether (and if so under what conditions) the introduction of a potentially risky technique is justified when safe alternatives, such as oocyte donation, are at hand. This requires a determination of the weight assigned to genetic parenthood (Peters, 2004; Graumann and Haker, 1998).

\subsubsection{Ethical aspects of reproductive embryo cloning in the context of mtDNA disease}

We concluded above that only NT type 3 , transplantation of the nucleus of a blastomere, amounts to reproductive embryo cloning. Using the pronuclei instead of the nucleus of a blastomere was crucial for the research license given to Newcastle 
University (to perform experiments with pronuclear transfer) (Human Fertilization and Embryology Authority [HFEA] summary decision RO153). After an initial rejection, the HFEA in second instance approved the Newcastle protocol as it concerns transfer of the 'pronuclei'. Cloning is thus avoided. As mentioned above, reproductive cloning is prohibited both by international organizations and many national laws. The current HFE Act (1990) and Reproductive Cloning Act (2001) for example prohibit reproductive cloning. 'The Appeal Committee accepted the view that the zygote at no stage contains a single nucleus. First it has two haploid pronuclei, then two diploid pronuclei, and then it enters mitosis' (HFEA summary decision RO153). Because the pronucleus is not the same as the nucleus, the HFE Act does not prohibit research involving pronuclei.

Data may also indicate that pronuclear transfer is more efficient than transfer in other stages (Taylor and Turnbull, 2005). Let us, however, do a thought experiment: what if the transfer of the nucleus of a blastomere, NT type 3, would be more efficient and safer than the transfer of the pronuclei? A relevant question then is whether the moral arguments to avoid reproductive cloning would outweigh technical efficiency and thus, perhaps, better results. Suppose the choice is made for NT type 3 and the nucleus of a blastomere is transferred. This may cause a dilemma. On the one hand, the creation of cloned children can be avoided by using only one blastomere of an embryo. The other side of the coin, however, is that the remainder of the embryo will be discarded. Therefore, one might opt to 'save' this embryo by using all its blastomeres. Perhaps up to four (or even more) clones may thus be created. One may have to choose between limiting the loss of embryos and avoiding the creation of genetically identical children (de Wert, 1999). What should be preferred: avoiding reproductive cloning, saving blastomeres or reproductive efficiency?

The principal question is whether it is categorically wrong to (reproductively) clone human beings. The moral debate on reproductive cloning is rather complex. Provided it would be safe, there may well be some moral reasons in favor of cloning. It may, for example, be used as an assisted reproductive technique in case of infertility, or (as in our discussion) to avoid genetic disease (Brock, 1998). Furthermore, some argue that the onus is on the opponents to produce arguments that reproductive cloning would be harmful (Dawkins, 1998). Others, on the other hand, dismiss reproductive cloning because it causes a 'yuk reaction' (Midgley, 2000), or because it would be unnatural. Other critics of reproductive cloning argue that it would violate a child's right to an open future, a concept coined by Feinberg (1980). As there will be a substantial time gap between the start of both lives, the clone has to live in the shadow of its 'original' (Holm, 1998; Buchanan et al, 2000). This argument, however, does not hold true for embryo cloning, at least insofar as twins thus conceived have a simultaneous start in 
life; this is not different from twins in the natural course of events (Bonnicksen, 1995; de Wert, 2000). Although they start their lives as genetically identical twins, they also begin their biography at the same time. They will thus be in ignorance of the future choices of the other (Buchanan et al, 2000). However, what if out of one single blastocyst different embryos would be made, cryopreserved, and be transferred sequentially? In that case, a clone would, again, have a (much) older twin. This may affect the sense of freedom of this child, or its right to an open future. It may have the feeling that it knows too much about itself, although this argument has been criticized for its genetic determinism (Brock, 1998; Buchanan et al, 2000; de Wert, 2000).

In any case, NT type 3 seems ethically more complex than the other variants of NT. Although the ethical debate has shifted primarily to research cloning (for obtaining stem cell lines), the context of mtDNA disease shows that the ethical debate on reproductive cloning remains important as well.

\subsubsection{Germ-line modification: the moral implications of modifying the mtDNA}

Another complex issue regards the debate on germ-line modification. Different types or arguments, both in favor and against, have been put forward. Consequentialist objections, for example, emphasize the impossibility to foresee the consequences of germ-line modification (Health Council of the Netherlands, 2001; Salvi, 2001). We already touched upon this when discussing the health and safety risks. Deontological objections bring to the fore that children born as a result of the intervention will not have the possibility to give consent. Furthermore, the child will inherit a manipulated genome, which can be perceived as a violation of its genetic integrity. Article 24 of the United Nations Universal Declaration on the Human Genome and Human Rights (1997) mentions that germ-line intervention is a practice that could be contrary to human dignity. However, human dignity is a controversial concept (Salvi, 2001), not further defined in the Declaration. Nevertheless, it is usually defined as having intrinsic worth and deserving respect. At the liberal philosophical level, human dignity is used to indicate that persons should always be treated as ends in themselves and never merely as means. Bearing this in mind, if germ-line modification is used to diminish suffering, would human dignity be adversely affected? One could even argue that human dignity is promoted, as modification of the human germ-line is the only means of preventing/treating a certain condition.

If the goal of the germ-line modification is clearly therapeutic, and safety can be guaranteed, we may have good reasons to assume that this future person would also have given consent. He/she will probably not regret that its genetic integrity has been violated. 
The ethical debate on germ-line modification focused entirely on altering the nuclear DNA. Neither OT nor NT would involve modification of the nuclear DNA. What about the ethics of modifying the mtDNA?

The general assumption is that mtDNA does not really constitute our genetic makeup; it does not influence our phenotype, as it only governs cellular energy production. Modification of essential or defining characteristics is considered to be ethically more problematic, because it determines one's identity or personality. Modifying the nuclear DNA is therefore often regarded more problematic than modifying the mtDNA. This is for example shown by the exceptions made in the Dutch Embryo Law and the proposals in the HFE Act (2007) review. It is also shown by the approval of the research license to the researchers of Newcastle University, where the Appeal Committee 'accepted that mtDNA is not associated with identity or predetermined characteristics of the individual' (HFEA summary decision RO153).

Whereas there seems to be a strong consensus that the mtDNA does not influences our 'character', little is known about the exact role and function of the mtDNA (Thorburn et al, 2001). One study suggests mtDNA involvement in cognitive functioning in mice (Roubertoux et al, 2003). Another study detects evidence of associations between mtDNA variation and susceptibility to alcoholism (Lease et al, 2005). How much certainty and evidence is there that mtDNA does not influence characteristics and physical appearance? It might be premature to conclude that modifying mtDNA is 'ethically irrelevant/neutral'. Some commentators note that we cannot exclude that the mtDNA is able to influence our individuality, since the mtDNA determines a part of the function of the mitochondria, and these on its turn influence energy production of the (neural) cells (Szebik, 1999). Similarly, others note that the mtDNA is usually ignored in policy debates about genetic engineering, 'on the basis of the weak assumption that it does not have significant phenotypic effects. But mitochondria do govern cellular energy production, and we are learning more about the downstream and far-reaching effects of that function on human physiology and (through the brain) on human behavior' (Parens and Juengst, 2001:397). Although this seems improbable in the case of mtDNA, there is always the possibility that a gene product has unexpected effects.

Clearly, further discussion on the acceptability of germ-line modification in general and modification of the mtDNA in particular is necessary before considering to introduce OT and/or NT for mtDNA disease in clinical practice.

\subsubsection{The moral implications of having three genetic parents}

OT and NT result in an embryo that inherits the vast majority of its DNA from the intentional mother and father, plus a proportion of mtDNA from the oocyte donor. To 
put this into perspective: the donor would contribute $0.1 \%$ of her DNA (the 37 mitochondrial genes) while the intentional parents contribute the remaining $99.90 \%$ of their DNA ( $\sim 24000$ nuclear genes). Strictly speaking, this may lead to the birth of a child with three genetic parents: the intentional parents delivering the nuclear DNA and the donor passing on the mtDNA (Health Council of the Netherlands, 2001). What are the moral implications of having three genetic parents?

The moral implications particularly relate to the interests of the child and the implications for the family. Some would consider NT to be unnatural or undermining the traditional family. This, however, are familiar objections to new reproductive technologies. In the early days of assisted reproductive technology, the impact of collaborative strategies (gamete donation, surrogacy) on the welfare of the child was already questioned. As Robertson (1994:13) puts it: 'Laboratory manipulation of embryos, the splitting of gestational and genetic parenthood, and prenatal screening risk producing children who are physically or psychologically injured by the techniques in question. Of special concern is the impact of children of several sets of genetic and social parents, some of whom the child will never know, which arise in the collaborative use of gamete donors and surrogates'. Collaborative reproduction is always more problematic than natural reproduction, as it introduces a third party into the usual situation of two-party parenthood and the 'traditional' genetic, gestational and social unity of reproduction might be separated.

As different collaborative strategies in the context of reproductive medicine are currently broadly accepted, the main concern regarding - and the novelty of -OT and NT is the mixing of mtDNA and the insertion of mtDNA of a third party into the cell. Some argue that this splitting of genetic motherhood should be considered the main ethical issue in the debate about OT and NT (Robertson, 1999). The psychological, legal and social consequences of having three genetic 'parents' are largely unknown. Moreover, it is debatable whether the oocyte donor, contributing her mtDNA, should be considered as a 'genetic parent' at all (Mertes and Pennings, 2008).

\subsubsection{Ethical aspects of oocyte donation in the context of mtDNA disease}

OT and NT require collaboration from oocyte donors in both the preclinical phase and in possible future clinical applications. Oocyte donation raises the same ethical questions as assisted conception in general or sperm donation in particular, such as the meaning of family values, sexuality, parenthood, gender relations and commodification (e.g. Cohen, 1996). However, as different assisted reproductive technologies are currently broadly accepted, we focus on another point of attention: the welfare of the oocyte donor. The first issue concerns genetic parenthood. Women donating oocytes for reproductive purposes in the context of mtDNA disorders will 
pass on their mtDNA to a child. However, given that oocyte donors normally donate both nuclear DNA and mtDNA, donation of the mtDNA will probably be more easily accepted (Robertson, 1999) and psychologically less burdensome.

A second issue concerns the risks and burdens of oocyte retrieval. The donating woman has to undergo ovulation induction and oocyte retrieval, like in normal oocyte donation (except for cases where an IVF patient donates some of her oocytes). Relevant issues are how the health and safety risks involved can be weighed properly, how autonomous decision-making can be guaranteed and how exploitation of women can be avoided (de Wert, 1999; Mertes and Pennings, 2007; Pennings et al, 2007a). It is proposed to treat women who donate oocytes for research purposes like other healthy research subjects in clinical trials (Mertes and Pennings, 2007). This proposal may be extended to those who donate oocytes for preclinical studies in the context of NT. Perhaps this could be the proper model for oocyte donation in the clinical phase as well. This depends on how the contribution of mtDNA is morally evaluated.

A third point concerns the difficult access to and the scarcity of donor oocytes. One could argue that, since oocytes are scarce, they should be used for reproductive purposes and not for research. Others may reply that the appropriate person to determine the destiny of oocytes is the donor herself. After all, some women will have fewer problems with donating oocytes for research than with donating for reproduction since they will not be confronted with a genetically related child.

Regardless of the purpose for which the oocytes are donated, one should pay attention to minimization of risks, avoidance of undue influence and guarantee of informed consent (de Wert, 1999; Mertes and Pennings, 2007; Pennings et al, 2007b).

\subsubsection{Nuclear transfer: down the slippery slope?}

In discussions about (bio)medical technology, the 'slippery slope' or 'thin end of the wedge' is an often-heard argument. Introducing or accepting a technology or application $A$ that in itself is not morally problematic, would be problematic if doing so makes it impossible (logically or empirically) to avoid the subsequent introduction or acceptance of another technology or application B that is morally unacceptable (van der Burg, 1992; Burgess, 1993; McGleenan, 1995; Health Council of the Netherlands, 2001). In the logical version of the slippery slope, accepting $A$ deprives one of valid arguments to reject $B$. The empirical version entails the prediction that accepting $A$ will lead to a climate of acceptance of $B$ as well. In both versions, the message is that anyone who regards B as undesirable, should reject A (Lamb, 1988; de Wert, 2005). In the case of NT, at least three slippery slopes can be discerned. 
The slippery slope towards germ-line modification of the nuclear DNA

This slippery slope argument runs as follows:

i. Once germ-line modification of the mtDNA is accepted, eventually modification of nuclear DNA will be accepted.

ii. Subsequently, once germ-line modification is accepted for therapeutic uses, it will lead to the application for non-medical uses, i.e. enhancement (Health Council of the Netherlands, 2001).

Possible arguments in favor of those concerns are that once germ-line modification is successfully applied, it may lead to a climate wherein other modifications of the germline indeed may be investigated. Furthermore, the line between medical and nonmedical uses is a troublesome one. On the other hand, arguments to refute this concern may be that no technique is currently capable of genetically altering characteristics of future human beings. Besides this, it is not the nuclear DNA that is altered during the procedure of NT, but the mtDNA. This may yield less ethical controversy than altering the nuclear DNA, which is, as we have discussed above, generally perceived as the locus of our characteristics and personality traits. Finally, this slippery slope argument assumes enhancement is wrong. Suppose NT indeed leads to enhancement, the question remains whether enhancement indeed is categorically wrong and therefore should be prohibited at all times, under all circumstances.

The slippery slope towards reproductive cloning

The second variant of the slippery slope argument concerns a gliding scale from NT to reproductive cloning.

i. Earlier, three types of NT were discerned, using an unfertilized, a half-fertilized or a fertilized oocyte (i.e. a blastomere of an embryo). Although the first and second types do not implicate cloning, some critics may argue they nevertheless should be prohibited: once they are accepted, they may well lead to the third type of nuclear transfer, which clearly is a type a reproductive embryo cloning.

ii. Subsequently, reproductive embryo cloning may eventually lead to reproductive adult cloning.

This slippery slope argument assumes that human cloning, more in specific reproductive embryo cloning, is to be condemned. Again, the fundamental ethical question to be addressed is whether it would always be, categorically, wrong to 
(reproductively) clone embryos. Even if that would indeed be the case, then it still does not automatically follow that accepting NT type 1 or 2 will inherently lead to NT type 3. NT type 3, on its turn, does not automatically have to lead to reproductive adult cloning.

The slippery slope towards misuse of NT

The third slippery slope regards the use of NT by other groups and with other aims than the prevention of mtDNA disorders. It may for example be used as an assisted reproductive technique for older, perimenopausal women seeking to have children (Parks, 1996; Eisenberg and Schenker, 1997; Barnett and McKie, 2004). Also in this slippery slope a debatable assumption is made, namely, that perimenopausal women should not reproduce in this way.

\subsubsection{Social justice in health care}

Another principle that needs to be scrutinized concerns the fair distribution of scarce resources. Justice and equal access to health care are among the most urgent issues in genetics and assisted reproductive medicine (Pennings et al, 2008). An argument against NT could be that mitochondrial diseases are rare, and mitochondrial diseases caused by a mutation in the mtDNA even more. NT therefore has a limited applicability and should not be given priority. Moreover, the development of the technique is time-consuming and expensive. Hence, it can be argued that it is inefficient and unfair to spent scarce health care resources on this type of research, even more when alternatives such as adoption and oocyte donation are available. Furthermore, although NT might prove to be successful in preventing the transmission of mtDNA disease, the mother (and other patients) will not benefit herself, as no curative treatment is available (Rubenstein et al, 1995). Money may be better spent on developing treatment, to help those people already suffering from a mitochondrial disease.

On the other hand, although the prevalence of mtDNA disease is incomparable with, for example cardiac disease, recent data identify mtDNA disorders as an important cause of genetic diseases (Taylor and Turnbull, 2005). The fact that not many people are known with mtDNA disorders may be related to the fact that there is no single hotspot among the mitochondrial disorders. Furthermore, many take the position that providing assisted reproductive and genetic technology to people at high risk of an affected child respects both the principle of justice and the principle of avoiding harm (Pennings et al, 2008). If we continue the analogy with cardiac disease, one might add that NT offers chances at the very beginning of life. Contrarily, many therapies and research concerning cardiac disease are centered at the end of life. The net gain 
of NT may be considerable. Moreover, the development and experiments with NT may yield interesting spin-off effects.

This difficult issue cannot be solved within the scope of this paper. Even if one argues that innovative techniques like NT should be developed, this still leaves the issue of reimbursement unsettled. The proper allocation of resources is actually a much wider moral and political issue that is and will be on top of the political agenda in the coming years.

\subsection{Conclusion}

In this paper, we identified the complex conceptual and normative issues that arise with regard to OT and NT to prevent mtDNA disorders. The first conceptual question we addressed is whether these techniques amount to germ-line modification. We concluded that both OT and NT encompass a type of germ-line modification in which only the mtDNA is changed. The consequences and the ethical significance of modifying the mtDNA need further scrutiny. The second conceptual question we addressed is whether NT amounts to human cloning. This depends on whether fertilized or unfertilized oocytes are used. Transferring the nucleus of a blastomere (NT type 3) indeed constitutes reproductive embryo cloning, making it ethically more complex than the other variants of NT. Normative issues concern, among others, the moral value of embryos, the health and safety risks for children conceived as a result of one of these techniques, the implications of having 'three genetic parents', the ethics of oocyte donation and the proper allocation of scarce resources. Further interdisciplinary debate and research should determine whether it is morally acceptable to introduce OT and NT in the clinic, and if so, under which conditions. 



\section{Chapter 7}

Ethics of nuclear transfer to prevent mitochondrial DNA disorders: revisiting the debate on germ-line modification and reproductive cloning.

Bredenoord AL, Dondorp WJ, Pennings G, de Wert G.

Submitted for publication 


\section{Abstract}

Preclinical experiments are currently performed to examine the feasibility of various types of nuclear transfer (NT) to prevent mitochondrial DNA (mtDNA) disorders. Clinical application would not only entail germ-line modification, but in one variant of the procedure also reproductive cloning (RC). Most objections against germ-line modification refer to modification of the nuclear genome, while in the application of NT considered in this paper the mitochondrial genome is the target of modification. Similarly, most objections against $\mathrm{RC}$ refer to reproductive adult cloning, while the relevant variant of NT to prevent mtDNA disorders would amount to reproductive embryo cloning. In this paper, we will first clarify these conceptual differences. We will then argue that if NT to prevent mtDNA disease were to become safe and effective, dismissing it because it involves germ-line modification and possibly also $R C$ is unjustified. 


\subsection{Introduction}

Mitochondrial DNA (mtDNA) disorders usually are severe disorders, caused by defects in energy production. Patients show a wide variety of symptoms, but generally the most energy demanding tissues such as the central nervous system, heart and skeletal muscles, liver and kidney are affected. Recent studies indicate that about 1 in 5000 people have, or are likely to develop mtDNA disease (Schaefer et al, 2008) and as many as 1 in 200 newborns carry one of the common mtDNA mutations (Elliott et al, 2008), making mtDNA disease one of the most common inherited neuromuscular disorders. As there is no curative treatment, helping carriers of mtDNA mutations to have healthy children has been a central focus of attention (Taylor and Turnbull 2005; Chinnery et al, 2006).

One future reproductive option to prevent the transmission of an mtDNA mutation from mother to child could be nuclear transfer (NT) (or 'mitochondrial gene replacement'). In case of NT to prevent mtDNA disorders, the mtDNA (which exists outside the nucleus, in the cytoplasm) is changed or replaced (Bredenoord et al, 2008c). This should result in healthy offspring with the nuclear genes of the parents, but without their mtDNA mutation (Gardner et al, 2007). NT does not entail genetic selection of an embryo or fetus; it is a type of genetic intervention or modification aimed at correcting the genetic cause of the disease, whereby the oocyte or embryo is reconstructed in its earliest stage. The exact procedure depends on the stage in which it is performed, which (in theory) could be three (de Wert 2000; Brown et al, 2006; Roberts 1999):

\section{Transfer of the nucleus of an unfertilized oocyte (NT type 1)}

The nuclear DNA of a donated oocyte from a healthy woman is removed. The nucleus of an oocyte of the prospective mother is then inserted into the enucleated donor oocyte. Subsequently, the reconstructed oocyte will be fertilized using the partner's sperm. The resulting embryo is then transferred to the prospective mother's womb.

2. Transfer of the pronuclei of the zygote (NT type 2)

An oocyte of the prospective mother is fertilized using her partner's sperm, as well as a donated oocyte of a healthy woman (the oocytes have to be in the same stage). When the oocytes are 'half fertilized', i.e. the sperm has penetrated the oocyte but no fusion has yet occurred, the two pronuclei are taken out of the donated zygote. Subsequently, the pronuclei of the intentional parents (containing their nuclear DNA) are transferred to the enucleated donor zygote. The resulting embryo is then transferred to the prospective mother's womb. 
3. Transfer of the nucleus of a blastomere of an embryo (NT type 3)

The nuclear DNA of a donated oocyte from a healthy woman is removed. An oocyte of the prospective mother is fertilized using her partner's sperm. The nucleus of a blastomere of the resulting embryo is then transferred to the enucleated donor oocyte. The resulting embryo is then transferred to the prospective mother's womb.

In (at least) two places in the world, NT is under preclinical development. Recently, researchers at the Oregon National Primate Research Center reported technical successes in NT type 1 (Tachibana et al, 2009). In a non-human primate model, Tachibana and colleagues transferred the spindle-chromosomal complex of a mature oocyte to an enucleated donor oocyte, resulting in three thus far healthy primates. Following the statements of the researchers (e.g. Kuehn, 2009), application for FDA approval to start the first human clinical trials will just be a matter of time. In the United Kingdom, experiments are performed to determine the feasibility of NT type 2 (Brown et al, 2006; Gardner et al, 2007). To our knowledge, NT type 1 and type 3 have not been applied clinically. A clinical application of NT type 2 has been reported once, resulting in a triplet pregnancy but no live birth (Zhang et al, 2003; Baruch et al, 2005).

No consensus exists regarding which type of NT would eventually have the best credentials, although the scientific community seems to focus particularly on the development of NT type 1 and 2 (e.g. Sato et al, 2005; Brown et al, 2006; Gardner et al, 2007; Tachibana et al, 2009). This, however, may be influenced by the ethical and political context: NT type 3 is considered to be a more controversial technique as it may amount to reproductive cloning $(\mathrm{RC})$. For example, when the Human Fertilisation and Embryology Authority (HFEA) had to decide on the research license for experiments on NT type 2, it explicitly mentioned that transfer of a nucleus of a cell of an embryo is prohibited (which, depending on the definition, could be perceived as RC; see below) (HFEA summary decision RO153). Although the current scientific interest seems focused on NT type 1 and 2, we do not want to exclude NT type 3 beforehand from our analysis merely because it would be politically more sensitive. After all, it is conceivable that NT type 3 turns out to be the most feasible or safest route. ${ }^{1}$

The rationale behind developing NT is that all existing reproductive options for carriers of mtDNA mutations have their limitations (Bredenoord et al, 2008a,b). NT may therefore be a valuable additional option. As having healthy children is for many

\footnotetext{
${ }^{1}$ One can for example imagine that smaller amounts of pathogenic mitochondria come along when only one nucleus is transferred (NT type 1 and type 3) instead of two pronuclei (NT type 2).
} 
people one of the most important desires in life, helping couples to fulfill this desire is a legitimate aim of a reproductive technique such as NT for mtDNA disease.

However, NT to prevent mtDNA disorders would seem to entail a form of germ-line modification and may in one variant of the procedure also imply reproductive cloning $(\mathrm{RC})$. As these are morally highly controversial issues, the question arises how these implications would reflect on the moral acceptability of NT to prevent mtDNA disorders. Here, it is important to note that most if not all moral objections that have been raised against germ-line modification refer to modification of the nuclear genome, while in the application of NT considered in this chapter the mitochondrial genome is the target of modification. Similarly, most objections against RC refer to reproductive adult cloning, while the relevant variant of NT to prevent mtDNA disorders would amount to reproductive embryo cloning (REC).

In this chapter, we will first clarify the applicability of the concepts of germ-line modification and RC. We will then examine whether and to what extent the main objections brought forward against germ-line modification and $\mathrm{RC}$ also apply in the context of NT to prevent mtDNA disease. We will presuppose the safety of the relevant forms of germ-line modification and $\mathrm{RC}$ as a preliminary condition, and focus on the objections of principle that share an unease regarding alteration or manipulation of the genetic make-up of future generations. ${ }^{2}$

\subsection{Germ-line modification}

We use the term germ-line modification to refer to any biomedical intervention that modifies the genome that a person can transmit to his or her child and the child's entire lineage (Chapman and Frankel, 2005). Not all genetic modifications affect the germ-line, but genetic modifications in the still pluripotent cells of the early embryo do. Not surprisingly, the concept is usually taken as referring to germ-line modifications of the nuclear genome, i.e. the DNA present in the nucleus of each cell. After all, this comprises $99,90 \%$ of the total amount of DNA (about 24.000 genes). However, each cell also contains a second genome, located in the mitochondria. These are tiny organelles in the cytoplasma surrounding the nucleus; they serve as the 'powerhouses' of the cell. The mitochondrial genome is extremely small, containing about $0.1 \%$ of the total amount of DNA (37 genes). The relevant genes

\footnotetext{
${ }^{2}$ We make no attempt to cover every possible objection as formulated in the extensive discussions about germ-line modification and RC. We selected the categorical objections and leave aside conditional issues, including safety issues - that would, in view of their importance, require a separate analysis. Furthermore, by focusing on germ-line modification and RC, we will not examine other possible objections to NT in the context of mtDNA disease (such as the use of embryos and the question whether NT results in three genetic parents and if so, how we should evaluate this. For a review we refer to Bredenoord et al, 2008c).
} 
are all involved in the cell's energy metabolism, whereas the nuclear DNA has a much wider range of functions. Transmission is also different. Unlike the nuclear genome, the mitondrial DNA is only transmitted maternally and in a less predictable (non-Mendelian) way. However, these differences (limited quantity, specific function, maternal and non-Mendelian transmission) do not change the fact that modifications of the mitochondrial genome in early embryonic cells can be transmitted to further generations. As this is the core of the concept of germ-line modification, there is no good reason for not also applying the concept to germ-line modifications of the mitochondrial genome.

\subsection{Reproductive (embryo) cloning}

Strictly speaking, cloning means the production of a genetic copy. We refer to reproductive cloning as all applications of human cloning that result in the birth of a child genetically identical to an embryo or a born individual (Bredenoord et al, 2008c). In contrast to reproductive adult cloning, where the clone is a 'copy' of a born individual, the original cell in the application of NT in question would be derived from an embryo. This would therefore be an instance of reproductive embryo cloning (REC).

Whereas NT type 1 and 2 do not involve REC, NT type 3 does. After all, NT type 3 entails the transfer of the nucleus of a blastomere to an enucleated oocyte. This results in two identical embryos. If an 8-cell blastomere would be used, up to eight blastomere nuclei may in theory be used to create identical embryos. If those embryos are subsequently transferred to the womb, this may result in the birth of children genetically identical both to the 'original embryo' and to each other. Following the above definition of $\mathrm{RC}$, a child is still to be considered a clone if it is the only individual ever born as a result from copying the 'original' embryo. Following a more narrow definition, this is not the case. According to this alternative definition of $\mathrm{RC}$, being a clone presupposes genetic identity with at least one other born individual. Whereas the first (broader) definition refers to the fact that a child is the result of genetic copying, the second looks at the consequence of the procedure: the birth of genetically identical individuals. According to the latter definition, whether NT type 3 implies REC depends on a) the number of embryos transferred and $b$ ) whether this leads to a pregnancy and live birth of at least two (identical) children. If only one cloned embryo is transferred to the womb, and the embryo does not split, this will not result in the birth of genetically identical children. If, however, more embryos are transferred, this may result in the birth of several clones. 


\subsection{Do moral objections against germ-line modification and RC also apply to NT for mtDNA-disease?}

As it is clear that NT aimed at preventing the transmission of mtDNA disease would be a form of germ-line modification and also that NT type 3 may indeed amount to $\mathrm{RC}$, we need to address the moral significance of these conceptual conclusions. In this section, we will briefly discuss four lines of moral objections that are brought against germ-line modification and/or RC. Where possible, a combined discussion of these two issues will be presented. The question to be answered is whether those objections amount to valid moral arguments against NT aimed at preventing the transmission of mtDNA disease.

\subsection{1. "It is repugnant and unnatural"}

A first objection against germ-line modification and RC is that they would be repugnant. According to Kass (2002), repugnance may be the expression of wisdom, beyond reason's power to articulate why something would be wrong. If, and insofar as, Kass means that emotional reactions should not directly be dismissed as they may point to important considerations that otherwise may be overlooked, then we agree. However, the second step should always be to subject these emotions to rational analysis, because otherwise it would imply a permit for moral prejudice (Nussbaum, 2004). The critical scrutiny of arguments is precisely necessary to prevent ethics to get bogged down in gut feelings. After all, our moral feelings may be misleading and put us in the wrong direction. For example, some people may have strong moral feelings about homosexuality or about the inferiority of some sections of the population. Ethical reflection and analysis may teach us that we do not have convincing arguments to hold and defend such a position (Gillon, 1999).

An adjacent variant of this objection is that germ-line modification and $\mathrm{RC}$ would be unnatural or artificial. There has been an extensive debate on this so-called argument of nature, with the dominant conclusion that it is fallacious. In the context of RC, Fukuyama (2002) has for example deployed this argument. He calls for an absolute prohibition of RC because it involves a highly unnatural form of reproduction. This seems rather thin as a counterargument, requiring further explanation of why unnaturalness would be morally problematic or relevant. Unnaturalness as such cannot be a valid argument - if we would take it seriously, we should stop practicing medicine (and many other human activities). The proponents of this objection will probably also regard germ-line modification and RC in the context of mtDNA disease to be repugnant and unnatural. For the reasons outlined above, we do not hold these objections to be decisive, neither in general, nor in the context of mtDNA disease. Nevertheless, perhaps those appealing to repugnance or 
unnaturalness have underlying, more fundamental arguments to reject germ-line modification and $\mathrm{RC}$ ?

\subsection{2. "We are creatures, not creators"}

The second line of objections against germ-line modification and RC expresses feelings of uneasiness regarding human intervention in the creation and shaping of future persons, albeit that different authors refer to different underlying reasons for not violating 'genetic integrity' (Lappé, 1991). This type of argument rests on the notion that 'we are creatures, not creators' (Cole-Turner, 2003: 195) and is particularly targeted at enhancement, i.e. the use of (genetic) technology aimed at adding, modifying or improving traits or characteristics not related to disease (Baruch et al, 2005). Many authors accept germ-line modification and RC for medical uses, while rejecting nonmedical uses. They however often insufficiently explain why a certain technique is acceptable in the first instance, while wrong in the second. Take for example Sandel (2007), who criticizes genetic engineering because it would express a stance of mastery and dominance to the world. 'Genetic engineering to create designer babies is the ultimate expression of the hubris that marks the loss of reverence for life as a gift' (Sandel, 2007:127). Hubris is a well-known theme in Greek tragedy, in which the main actor, by challenging the gods or their laws, is inevitably heading to his downfall. Similarly, by denouncing genetic engineering a type of hubris, the objection is that these are acts of excessive pride or arrogance that are likely to lead to nothing good.

One could of course argue here that Sandel's objection is solely targeted at enhancement, not at medical uses such as NT for mtDNA disorders, but that is exactly the problem: there is a tension between asserting that genetic engineering is a type of hubris and at the same time allowing germ-line modification for the prevention of serious medical disorders. If genetic engineering is a type of hubris, does it matter for which purpose the technique is used? It is one of two things: either one conditionally accepts germ-line modification and $\mathrm{RC}$ and discusses the proper mode and scope of their use, or one dismisses all uses as forms of hubris.

There are two further reasons why the above objection is not convincing. First, the validity of the view depends on a contestable normative anthropology. An anthropology that contends that people should not explore the limits of their knowledge and capacities, and that seems to summon people to know their place. If one does not share this specific portrayal of mankind, this argument is not convincing. Contrarily, we would rather encourage people to explore new technology and new paths for curing disease rather than invoke distrust of technology. 
Second, in so far as this objection asserts that we eventually would lose our reverence for life as a gift, it may actually be interpreted as a slippery slope argument. The question is whether the dreaded position at the end of the slope indeed would be morally unacceptable. Would it be problematic that people would not perceive life as a gift? And does seeing life as a gift automatically imply that one should not alter it? If there would be a slippery slope here, the first steps have been set long ago, with the introduction of ART.

\subsection{3. "It is a violation of human dignity"}

A third line of objections against germ-line modification and RC holds that they violate human dignity. Dignity is a much contested and vague concept and no agreement on the meaning of the term exists (President's Council, 2008; Van der Graaf and Van Delden, 2009). It is mostly used to indicate that human beings have intrinsic moral worth, but this forces us to specify what gives human beings this intrinsic worth (Kass, 2008). Since human dignity comes in such competing versions, it is understandable that authors have dismissed the concept as useless or vague (see for an overview Beyleveld and Brownsword, 2001; Van der Graaf and Van Delden, 2009). We will nevertheless try to scrutinize this objection by using two opposed conceptions as formulated by Beyleveld and Brownsword (2001): human dignity as empowerment or as constraint. These two conceptions make clear that human dignity can be deployed both in support of autonomy and as a constraint on autonomy (Beyleveld and Brownsword, 2001).

The first conception, human dignity as empowerment, is particularly used in the post Second World War international treaties on human rights. The intrinsic dignity of human beings is the foundational idea, the justification for the recognition of human rights (Beyleveld and Brownsword, 2001). As Caulfield and Brownsword (2006:72) have put it: 'In this reading, human dignity is an engine of individual empowerment, reinforcing individual autonomy and the right to self-determination'. In so far as human dignity is equated with the capacity for autonomous action, this understanding of human dignity will be discussed in the next section, when we address autonomy and the right to an open future.

The concept of human dignity is also and increasingly used as a justification for condemning practices such as germ-line modification and RC. Recent international treaties have used human dignity in this way. The Council of Europe for example states that the deliberate creation of genetically identical human beings (i.e. $R C$ ) is contrary to human dignity (CoE, 1998). Those treaties deploy human dignity as an argument to reject $\mathrm{RC}$ or germ-line modification, but they do not further explicate how 
human dignity is to be understood or what its foundations are. It is, in our opinion, important that these treaties further underpin why and how they use human dignity, in order to enable a moral dialogue.

Some authors do explain why human dignity should be deployed as a regulatory constraint. Fukuyama (2002), for instance, views human dignity as based in the common nature, or essence, that human beings share. This essence, which he calls factor $X$, is the sum of all typically human characteristics such as morality, emotions, language et cetera. According to Fukuyama, this essence is threatened by biotechnology. As human beings have proven to be particularly interested in the utilitarian goal of avoiding pain and suffering, he expects us to use genetic engineering to meet this goal at the cost of other human aims and qualities. We may for example try to reduce aggressiveness, but the price we may have to pay is that this results in people with a flatter character. Or we may succeed in prolonging life, but an increased longevity may affect the quality of life. In the end, Fukuyama states, biotechnology will involve trade-offs in which human complexity and human nature are at stake.

To alter gene A may indeed have (unforeseen) side-effect B. If genetic engineering were to be deployed on a large scale, human nature may perhaps indeed be threatened. But how does Fukuyama picture people deploying RC and germ-line modification on a massive scale? This is currently rather far-fetched and also way beyond the application of germ-line modification and $\mathrm{RC}$ in our discussion, namely to prevent the transmission of an mtDNA mutation. It is important to emphasize the context in which germ-line modification and RC would occur. The mtDNA, with its 37 genes, is not characterized by the genetic variation typical of the nuclear DNA. It seems therefore less sensitive to the threat as presented by Fukuyama. Furthermore, Fukuyama already himself answers how the possible negative effects of genetic technology should be encountered: by preventing misuse of the technology and by imposing appropriate conditions for its use, not by rejecting the technology at such. Another difficulty is that, although he makes a distinction between the use of biotechnology for therapy and for enhancement, Fukuyama fails to explain why human nature will not be threatened in the first application while it would when used for enhancement. Furthermore, it is also not clear why he needs his concept of 'essence' or 'nature' to underpin why biotechnology should be regulated. We agree that this should be the case, but not so much because of speculative reasons such as the threat to human nature, but more so to guarantee safety and prevent premature uses. 


\subsection{4. "It is a violation of the child's right to an open future"}

A fourth line of objections is that germ-line modification and RC would violate - what Feinberg (1980) in another context coined - the child's right to an open future. Although debate is possible about the interpretation of this concept, taking it as a negative anticipatory autonomy right would be the most relevant and appropriate in our discussion. This means a right to have one's future options kept open until one is capable of making one's own decisions. We differentiate here between germ-line modification and $\mathrm{RC}$ as the arguments run slightly differently.

Does germ-line modification violate a child's right to an open future?

Habermas (2003) has argued that genetic modification may entail a prejudgment of specific life projects, which may imperil autonomy. It threatens the child being the author of its life, because others have already framed it, or the child could have the feeling he/she is tailored towards its parents' expectations. In other words: by choosing the genetic make-up of a child, its right to an open future is violated. As we endorse the position that in a pluralist liberal society one should allow people to be the authors of their lives (Dekker, 2008), it would indeed be problematic when germline modification would restrict a child's options in life, or when it would predetermine a child towards specific life projects. It is therefore important to assess whether germline modification of the mtDNA may indeed potentially threaten the child's right to an open future.

Does modification of the mtDNA violate a child's right to an open future?

The moot point of the argument is the fear that it would become possible to alter presumed 'essential characteristics' of the genetic make-up of future generations. For this reason, germ-line modification of the nuclear DNA has led to more ethical controversy than modification of the mtDNA. The argument would be that the nuclear DNA contains the ingredients for our characteristics, not the mtDNA (e.g. Rubenstein et al, 1995; Sato, 2005). The HFEA for example explicitly starts from the premise that mtDNA is not associated with 'identity or predetermined characteristics of the individual' (HFEA summary decision RO153). However, the assumption that modification of the mtDNA is ethically less problematic than modification of the nuclear DNA because it would not involve the possibility of altering essential characteristics is contestable for two reasons.

Firstly, although the dominant view in science is that only the nuclear DNA contains the ingredients for our characteristics, this is not a fully settled issue. Usually, mtDNA is not considered important for our individuality, also since thousands of people have more or less identical mitochondria. The mtDNA would only govern cellular energy 
production. However, different authors have questioned the scientific assumption that the mtDNA is not associated with essential characteristics. Some studies, although not uncriticized, suggest associations between the mtDNA and cognitive capabilities (Roubertoux et al, 2003; Moreno-Loshuertos et al, 2006). Other authors have remarked that mitochondria may have a significant but unknown biological function other than energy production (Nagao et al, 1998). In fact, little is known about the exact role and function of the mtDNA (Thornburn et al, 2001) and little is known about the significance of altering the match between nuclear and mitochondrial complements (Bonnicksen, 1998; Bowles et al, 2007; St John et al, 2004; Poulton et al, 2006). Although perhaps hypothetical, the mtDNA may influence nuclear gene expression and this, in turn, may influence essential characteristics of the resulting child (Szebik, 1999; Parens and Juengst, 2001).

A second reason for criticizing the assumption that modification of the mtDNA would not involve the possibility of altering essential characteristics, is that none of the key terms used to make this distinction (such as 'essential characteristics' and 'identity determining characteristics') are ever precisely defined. The HFEA for example does not explicate how it understands 'identity or predetermined characteristics of the individual'. Although it is remarkable that key concepts are not defined, this should not come as a surprise, because what is it that separates one person from another? And what exactly constitutes our identity?

Does modification of the mtDNA affect the identity of the future child?

A relevant question here is whether germ-line modification leads to the birth of a different person (Ossorio, 2003). In case of other reproductive techniques, such as preimplantation genetic diagnosis (PGD), one chooses one embryo over another for implantation. If one decides to transfer embryo $A$ instead of embryo $B$, this clearly leads to the birth of a different person. In case of germ-line modification, this is less clear. The embryo is not substituted but modified: does this affect the identity of the person who is born?

Although it is beyond the scope of this chapter to create a coherent account of identity, conceptual clarity may come from Parfit (1984). He discerns two types of identity: qualitative identity and numerical identity. Qualitative identity means that two persons or two objects are exactly alike (Parfit uses the example of two white billiard balls). Numerical identity means that two persons or objects are one and the same (one thing or person rather than two). For example, Barack Obama is numerically identical to the President of the United States: they are one and the same person. Identical twins may be qualitative identical, meaning they are exactly alike. Numerically, though, they are different: they are two different persons. In some 
circumstances, it is meaningful to say that a person has qualitatively changed, but not numerically. A person who has had an accident may be qualitatively changed, meaning that this person's character has changed - when he changes a lot, he becomes a different person in a qualitative sense (DeGrazia, 2005). Numerically though, it is the same person - once we exist, our numerical identity is quite robust through change (Parfit, 1984, DeGrazia, 2005).

When a gamete or the early embryo is modified, debate is possible about whether this changes the numerical identity of the future person: will germ-line modification result in the same human being, or does it result in a distinct human being as would otherwise have originated (DeGrazia, 2005)?

This metaphysical question, however, is outside the scope of this chapter. Here, it is sufficient to say that a germ-line modification will at least affect the qualitative identity of the future person. Ossorio (2003) uses the speculative example of germ-line modification for Lesch-Nyhan syndrome, an early-onset disease characterized by mental retardation, self-mutilation and muscular and neurological abnormalities. If one would replace the gene underlying Lesch-Nyhan syndrome in the early embryo, the person who would be born would theoretically have a normal IQ, a normal life expectancy, no muscular and neurological abnormalities et cetera. Ossorio concludes that this person's identity has been changed, as it will have a different life experience and a different character. It would be difficult to maintain that a germ-line modification does not somehow affect the (qualitative) identity of the future person. Even if mtDNA only has a basic cellular function, then it is still meaningful to say that germ-line modification of the mtDNA is likely to change the (qualitative) identity of the future person. After all, a person without a mtDNA disease will have a different phenotype, a different life experience, a different biography and perhaps also a different character. This means that the dichotomy between modification of the nuclear DNA and modification of the mtDNA is untenable from this perspective: no matter whether one modifies a (pathogenic) nuclear gene or a (pathogenic) mitochondrial gene, the identity of the future person will be changed.

However, even if a germ-line modification of the mtDNA is likely to alter the identity of the child, a clinical application of NT to prevent mtDNA could still be compatible with the position that one should not violate the child's right to an open future. To prevent that a child is predetermined towards a specific plan of life, it seems reasonable to only allow modification that broadens so-called 'general purpose means' (Buchanan et al, 2000), which are useful for nearly all plans of life. In other words, we should only allow genetic modifications of which we can assume that they give children traits that are useful for all conceptions of a good life (Dekker, 2008). Although debate is 
possible about what general purpose means exactly are, being healthy should clearly be included ${ }^{3}$. Health, after all, is a sine qua non for many (though not all) plans of life. Disabilities and disorders often (though not always) mean that people have less good lives than they would have had (Glover, 2006). It is reasonable to assume that a child with a serious neurological or muscular disorder will experience the disease (and the underlying mtDNA mutation) as a barrier for many life plans. This is not to say that these children cannot have satisfactory lives. But if germ-line modification can safely avoid the frequently devastating effects of the mtDNA mutation, it is reasonable to presume that the future child will have more options in life. Interestingly, also Habermas would probably allow this application, as he allows germ-line modification 'for the goal of avoiding evils which are unquestionably extreme and likely to be rejected by all' (Habermas, 2003:43).

To conclude, the mtDNA modification is likely to alter the identity of the child, but it does not necessarily limit the child's future options. On the contrary, the child may even have more options in life. Whether the child's improved health compensates for the drastic intervention is an assessment that only can be made case-by-case, depending on the likelihood of developing disease symptoms, the expected seriousness of the disease and the expected 'costs' in the trade-off (including the risks of the intervention).

\section{Does $R C$ violate a child's right to an open future?}

Regarding $\mathrm{RC}$, the strong version of this argument counsels that a child's right to an open future may be violated in the sense that the clone has to live in the shadow of its 'original'. The child's right to ignorance about its future would be violated, as the clone, being genetically identical to someone else, knows too much about itself (Jonas, 1974). The strong version, however, is untenable: it rests on too strong an account of genetic determinism. Genetic identity does not equal personal identity (Gillon, 1999). The clones would not be identical persons, as personality and character are not determined by genetics alone, but by a complex interaction of social, environmental and indeed genetic factors (British Medical Association, 1999; Buchanan et al, 2000; de Wert, 2000). Currently existing monozygotic twins show a broad range of similarities and dissimilarities, thus demonstrating that sharing identical genotype and environment does not result in identical persons (Harris, 1997). The weaker version, however, should be taken more seriously. This version of

\footnotetext{
${ }^{3}$ Whether and to what extent the concept of 'general purpose means' would also be appropriate for assessing the acceptability of modifications intended to enhance human traits is outside the scope of this chapter.
} 
the argument counsels that the child's right to an open future may be violated as the child has the feeling that it knows too much about itself.

It is useful to emphasize here that this argument is primarily developed in the context of reproductive adult cloning. What about its validity in the context of REC? This argument would in any case not hold true in two situations regarding REC. The first exception would be when only one child is born. Following the more narrow definition of $\mathrm{RC}$, the child is not to be regarded as a clone in the first place, which means that the question whether cloning would affect the child's open future does not even arise. Although in terms of the broader definition the resulting child would indeed be a clone, this has no implications for the moral acceptability of NT type 3, as the child will not have to live with another 'co-clone'. Being alone as a clone, the child will not have to suffer from living in the shadow of a genetically identical individual. The second exception is when the twins, conceived by means of REC, are born simultaneously; this would not be different from identical twins in the natural course of events (which can be considered as naturally occurring clones) (Bonnicksen, 1995; British Medical Association, 1999; De Wert, 2000). Although they start their lives as genetically identical twins, and although they may share many characteristics, they also begin their biography at the same time. They will thus be ignorant of the future choices of the other (Buchanan et al, 2000).

In our opinion, the main ethical bottleneck regarding REC concerns the possible asynchrony between two (or more) cloned children. What if out of one single blastocyst different embryos would be made, cryopreserved for future use, and transferred later? This could result in a 'delayed twin' (British Medical Association, 1999): a clone with a (much) older twin-brother or -sister (or several identical sibs). This may indeed affect the sense of freedom of the delayed twin. The delayed twin may experience psychological distress by growing up in the shadow of an older sibling. After all, as the clones will grow up in the same family, they will share not only identical DNA but also a similar environment. If the delayed twin is transferred shortly after its older sibling, they may differ only one or two years in age. The delayed twin may (wrongly) think that the choices and the course of life of its older twin is an inevitable path. Or perhaps the parents may have this perception. We do not think that a right to an open future is violated merely because a clone or his/her surrounding believe that the clones future is already determined, when that belief is false and based on an erroneous genetic determinism (Brock, 2002). Nevertheless, the clone may still experience psychologically stress.

The first question, then, is whether the delayed twin(s) would have an acceptable quality of life. One could argue here, following Parfit (1984), that as long as a 
child/clone does not have a life so awful that it would be better off dead, bringing it into existence will not harm the child. This is because the alternative for this child would have been non-existence (this child could only be born as a clone). Insofar as violating the interests of the future child is concerned, this line of reasoning is generally accepted. There is, however, less consensus about whether this settles the issue with regard to justifying assisted reproduction. Although no harm may be done in the sense of a violation of the child's interests, this does not make it a matter of moral indifference what quality-of-life the child can be expected to have (Arras, 1990; Steinbock and McClamrock, 1994; Parker, 2005; Glover, 2006; Bredenoord et al, $2008 b)$. It is still meaningful to say in a more general sense that bringing a child into the world that will seriously suffer is to cause avoidable harm. The reasonable welfare standard, which we defended earlier as the best way to take into account the welfare of the child, allows ART (RC and NT included) insofar as there is 'no high risk of serious harm' for the resulting child (de Wert, 1998; Pennings, 1999; Bredenoord et al, 2008b). This standard entails the view that for ART to be justified, the child to be must have a reasonable chance of an acceptable quality of life.

The question is whether the delayed twin would be at high risk of serious psychosocial harm. Clearly, no hard evidence exists. Nevertheless, we doubt whether the delayed twin will psychologically suffer to such an extent that this provides a contra-indication in light of the reasonable welfare standard. Whether the twins differ one year or 20 years in age may also influence the psychological wellbeing of the delayed twin, but also this is speculative. Some have brought forward the view that the delayed twin may even have an advantage, because he or she may learn from the older twin (Brock 1998). In any case, to conclude that RC would result in a high risk of serious psychological harm for the child is premature. Nevertheless, if people in the daily surroundings of the clones would treat them as copies or negatively approach them, then psychosocial harm would become a self-fulfilling prophecy. Not merely because the younger twin would feel restricted by the older brother or sister, but because it would feel restricted by the expectations and (negative) feelings in his/her surroundings. So, this objection may become true if we allow it to be true.

Although it is understandable that the scientific community has focused on NT type 1 and 2, as these stages do not amount to RC, NT type 3 may be acceptable as well, even when a delayed twin would be brought into the world. However, it remains impossible to know the precise psychosocial consequences for the resulting children. Would it therefore be better to avoid the existence of delayed twins as much as reasonably possible? This could be done by transferring several cloned embryos 
simultaneously, or by starting a new (stimulation/NT) cycle if a couple desires to have another child.

\section{Avoiding delayed twins by multiple embryo transfer}

By transferring several cloned embryos simultaneously, the resulting children will start their lives simultaneously. Although speculative, one may for reasons outlined above expect that this causes no psychological harm (for it is similar to naturally occurring monozygotic twins). Transferring all blastomeres of the embryo may in theory result in up to 8 children. What about the transfer of 2, 3 or 4 embryos? The dilemma would then be as follows. Transferring several cloned embryos simultaneously may on the one hand avoid possible psychological problems, but on the other hand seriously increase medical complications (for the twins as well as the mother) and parental stress (and this may in turn cause stress in the children) (Braude, 2007). Furthermore, data indicate that the quality of life of children born after ART increase if they are born one at a time (Fauser et al, 2005). With regard to regular IVF, a strong consensus exists that, because of the health risks of multiple pregnancies, no more than two embryos should be transferred - and the current tendency in IVF is even heading towards single embryo transfer (Braude, 2007).

Whereas the psychological risks for delayed twins are speculative, the psychological and health risks in the context of multiple pregnancies are known to be substantial. When balancing these, it is preferable to avoid the known and substantial risks and to transfer only one or maximally two cloned embryos simultaneously.

\section{Avoiding delayed twins by starting a new cycle}

Choosing to transfer only one or two cloned embryos leads to the question what should be done with the remaining blastomeres or cloned embryos. One option would be to cryopreserve all cloned embryos. The question of course is whether cryopreservation of these reconstructed embryos would be feasible (the cryopreservation of biopsied embryos for example has not been very successful so far), but let us suppose that it would. The primary reason for doing this would be to avoid having to start a new stimulation/NT cycle if after unsuccessful transfer the couple would want a further try. But what if the procedure was successful and two years later the parents return with the request to help them expand their family? One option may then be to thaw and transfer one of the cryopreserved embryos. The other option would be to start a new stimulation/NT cycle. The dilemma here is whether avoiding delayed twins compensates for the efforts, costs and risks of starting a new stimulation/NT cycle. When deciding about this, we also have to balance the known risks and burdens of ovarian stimulation versus the speculative psychological risks for the delayed twins. As no further data regarding NT are at 
hand, we do not feel adequately equipped to make this appraisal at this moment. It is currently sufficient to conclude that although we do not consider the fact that NT type 3 involves $\mathrm{RC}$ to be a decisive objection, it may in practice lead to complex ethical considerations. Other things equal, this constitutes an argument to at least initially focus on the development of the other types of NT.

\subsection{Conclusion}

In this paper we discussed the moral acceptability of germ-line modification and $\mathrm{RC}$ in the context of NT to prevent mtDNA disorders. Modification of the mtDNA is often considered less controversial than modification of the nuclear DNA, as the presumption is that it is the nuclear DNA that contains the ingredients for our characteristics. Modification of the mtDNA would not affect the identity of the future child. However, even if the mtDNA only has a basic cellular function then it is still meaningful to say that a germ-line modification of the mtDNA is likely to change the (qualitative) identity of the future person. This means that the dichotomy between modification of the nuclear DNA and modification of the mtDNA is untenable from this perspective: no matter whether one modifies the nuclear genome or the mitochondrial genome, the identity of the future child will be changed. Nevertheless, as health is a sine qua non for many plans in life, modification of the mtDNA would still be an acceptable application of germ-line modification.

Regarding REC, we conclude that there are no a priori arguments that render REC morally unjustified. If NT type 3 results in the birth of one child, then (other things equal) this type of NT is ethically comparable to NT type 1 and 2. The simultaneous birth of two (cloned) twins should not be considered morally unjustified either. Although bringing into existence a delayed twin raises concerns about a child having to grow up in the shadow of an older clone, it remains speculative whether this would lead to psychosocial harm. The argument would therefore not amount to a decisive objection against NT type 3. On the other hand, the fact that the relevant concern is not completely implausible either does provide a prima facie reason to focus at least initially on the further development of NT type 1 and 2. A further reason for this is that NT type 3 may in clinical practice lead to a complex appraisal of the relative weight of avoiding delayed twins versus avoiding a multiple pregnancy.

We conclude that the fact that NT with the aim of preventing the transmission of mtDNA disease would involve germ-line modification and possibly also RC cannot convincingly be construed as categorical moral objection against the possible use of this technology. Of course, the overall acceptability of using NT for this purpose would also depend on the safety and efficacy of the procedure. 


\section{Chapter 8}

Avoiding transgenerational risks of mitochondrial DNA disorders: a morally acceptable reason for sex selection?

Bredenoord AL, Dondorp WJ, Pennings G, de Wert G. Human Reproduction, in press 


\section{Abstract}

In this chapter we discuss sex selection not intended to help a couple avoid having a child with a severe genetic disorder, but to avoid possible health risks further along the line of generations. Sex selection may be put to this use in the context of preventing mitochondrial DNA (mtDNA) disorders by means of preimplantation genetic diagnosis (PGD) and possibly in the future also through nuclear transfer (NT; also known as mitochondrial gene replacement). A relevant analogy can be found in the context of PGD for X-linked diseases, where sex selection against healthy female carrier embryos would have the same twofold purpose of a) avoiding difficult reproductive decisions for the future child and b) avoiding transmission of the mutation to a possible third generation.

Because sex selection would still be done for reasons of health, this application should not give rise to the moral concerns associated with sex selection for nonmedical reasons. However, the proportionality of adding the relevant procedures to PGD or NT is a relevant concern. We discuss post- and preconceptional sexselection strategies. We conclude that if PGD is already part of the procedure, either as the central technology or as a back-up test after NT, preferentially transferring male embryos could in principle be a morally acceptable way of reducing possible burdens and risks. To start an IVF/PGD-cycle especially for this purpose would be disproportional. The alternative approach of preconceptional sex selection may be morally justified as a means to increase the chances of obtaining male embryos. 


\subsection{Introduction}

Whereas sex selection is highly controversial for nonmedical uses, it is generally accepted for medical reasons (e.g. Health Council, 1995; ASRM, 1999; Pennings, 2002). This is usually defined as sex selection to avoid the birth of a child with a severe genetic disorder. Actually, preimplantation genetic diagnosis (PGD) was first introduced to select female embryos in the case of X-linked disorders (Handyside et al, 1990). In this chapter we discuss sex selection not intended to help a couple avoid having a child with a genetic disorder, but to avoid possible health risks further along the line of generations.

The reason for bringing up this issue is that sex selection may be a helpful additional measure in the context of preventing the transmission of mitochondrial DNA (mtDNA) disorders. As there is no curative treatment, helping carriers to have healthy children has been a central focus of attention. New techniques to achieve this are PGD and possibly in the future nuclear transfer (NT), also known as mitochondrial gene replacement. When applying PGD for mtDNA disorders, it is conceivable that only affected embryos are available for transfer. In that case, PGD would only be undertaken as a 'risk-reducing' strategy (Bredenoord et al, 2008b). Provided, however, that only embryos with a mutant load (far) below the threshold for disease expression are transferred is it very likely that the child will be free of the relevant disease (Bredenoord et al, 2009).

In case of NT, a donated oocyte is enucleated and replaced with the nuclear DNA from a woman carrying an mtDNA mutation. To obtain these oocytes, both the oocyte donor and the recipient woman are required to undergo hormone stimulation and oocyte retrieval. The nuclear transplantation could be performed before and after fertilization. In the first case, the nucleus of an (immature or mature) oocyte of the prospective mother is inserted into the donated oocyte. This reconstructed oocyte is then fertilized with the prospective father's sperm. In the latter case, NT would be performed after fertilization, using either the pronuclei of the zygote or the nucleus of a blastomere of the embryo (Roberts; 1999, Brown et al, 2006; Bredenoord et al, 2008c). The resulting embryo is subsequently transferred to the prospective mother's womb. Theoretically, this procedure should lead to a child without mutant mtDNA. Whether this can be completely achieved in practice, however, is not yet clear. Preclinical experiments have suggested that it may be difficult to avoid small amounts of affected mitochondria coming along with the oocyte, the pronuclei or the nucleus of the recipient woman (Sato et al, 2005; Nakada et al, 2008). However, the recently published data from the first application of NT for this purpose in non-human primates were reassuring in this respect (Tachibana et al, 2009). In this study, it was shown that the procedure can be accomplished without significant mtDNA carryover 
from the prospective mother's oocyte (Tachibana et al, 2009). However, it remains to be seen whether a clinical application of NT would indeed result in a child free of mutant mtDNA. As the authors observe, a mutant load of $3 \%$ would be undetectable, meaning that heteroplasmy may still be present.

Earlier we defended the view that medically assisted reproduction is acceptable as long as there is no high risk of serious harm for the resulting child (de Wert, 1998; Pennings, 1999; Bredenoord et al, 2008b). From this perspective, both PGD and NT for mtDNA disorders could be morally acceptable as long as only embryos with a mutant load below the threshold to disease expression are transferred (Bredenoord et al, 2009). The fact that even under this condition there may still be residual health risks is not a morally compelling reason to refrain from PGD or NT for mtDNA disorders. After all, it is unlikely that the resulting child will be exposed to a high risk of serious harm.

However, an important remaining concern is the possibility of recurrence of mtDNA disease in the child's offspring (Tachibana et al, 2009). Due to the existence of a genetic bottleneck (Poulton and Marchington, 2002; Cree et al, 2008), a low-level mutant load in the second generation (the child to be conceived through PGD or NT), may rise to higher levels in the third generation (the couple's grandchildren). In the light of this concern, there is an important difference between further reproduction by either male or female offspring. As mitochondria are transferred maternally, male offspring will not pass on their mutant DNA to the next generation. This leads to the question whether the avoidance of transgenerational health risks provides a morally acceptable reason for sex selection.

\subsection{The case for sex selection}

The fact that a low mutant load in their child may increase in their possible grandchildren may well be a cause of concern for the prospective parents. They may argue that both the possible burden of difficult reproductive decisions for the second generation and the health risks for a possible third generation are important enough to be prevented if reasonably possible. For most mtDNA diseases, sex selection will not reduce the health risks for the second generation (these risks are the same in both sexes). However, it will allow second generation males and their partners to reproduce with confidence. After all, they will not be confronted with the same difficult decisions their parents had to face. As males do not transmit the mtDNA mutation, a possible third generation will not carry the mtDNA mutation. For the couple to avoid transmission of an mtDNA disease, this may be a reason for preferring a son.

Clearly, the reproductive burdens and possible health effects that adding sex selection to PGD or NT would help to avoid, are uncertain. It is neither self-evident 
that second generation females want to have children (although most women do), nor certain that the mutant load will indeed increase in their offspring. It is also true that these women will themselves have the option of using PGD or NT (or even better strategies available at that time) to avoid having an affected child, or that a cure or procedure to prevent or ameliorate mtDNA disease has become available.

A helpful analogy can be found in the context of PGD for X-linked diseases (de Wert, 2005). Whereas all daughters of a male patient affected by an X-linked disease, for example haemophilia, will be obligate (healthy) carriers, all his sons will be unaffected non-carriers. Sex selection against healthy female carrier embryos would in such cases have the same twofold purpose of a) avoiding difficult reproductive decisions for the child and b) avoiding transmission of the mutation to the third generation. Notwithstanding the uncertainty of these effects, it has been argued that sex selection for this double purpose would be acceptable in principle in such cases (de Wert, 2005).

Can we draw the same conclusion with regard to sex selection in the context of PGD or NT for mtDMA disease? It may be objected that the analogy is incomplete. The predictability and severity of the transgenerational health effects to be avoided through sex selection are considerably more certain in the case of X-linked disorders than in the case of mtDNA disorders. Of course, it is equally uncertain whether any daughter of a man with haemophilia will reproduce. But if she does, $50 \%$ of her sons will be haemophilia patients like their grandfather. By contrast, it is far more difficult to predict whether and to what extent a possible grandchild of a couple that used PGD or NT for mtDNA disease may be affected by a transgenerational rise in mutant load. We agree that the case for additional sex selection is indeed less obvious here than where PGD for X-linked disease is concerned. But also in this context, adding this step is still defendable in principle. After all, chances that a possible daughter will want to reproduce are high and any remaining uncertainties about the possibility of her having a seriously affected child may well lead to difficult reproductive decisionmaking.

\subsection{Sliding towards nonmedical sex selection?}

Sex selection is generally accepted as a way of avoiding the transmission of serious sex-linked diseases, but regarded as morally problematic if requested for nonmedical reasons, e.g. because the parents want a male heir to carry the name of the family. Moral objections refer to gender discrimination and undesirable demographic and social effects of large scale application (Health Council, 1995; ASRM, 2001; Pennings, 2002). Sex selection for the purpose discussed in this chapter differs from the traditional understanding of sex selection for medical reasons (which we will refer 
to as 'the medical model'), in that it is not aimed at avoiding health risks in the child to be conceived in the current procedure. To the extent that it is aimed at avoiding health risks, these will only materialize (if at all) in a possible third generation, whereas with regard to the child to be conceived the aim is to avoid psychosocial burdens of reproductive decision-making, not health risks in the strict sense. Although sex selection for these purposes would not fall within the bounds of the medical model, neither does it seem correct to present it as an instance of sex selection for nonmedical reasons. As in the above-mentioned analogy from the context of PGD for X-linked disease, we are dealing here with an intermediate form of sex selection: neither medical in the strict sense of the medical model, nor nonmedical as referring to parental preferences that have nothing to do with health or disease (de Wert, 2005).

Although the concerns raised by those nonmedical reasons do not apply to sex selection for the intermediate reasons discussed in this chapter, it can be objected that by accepting sex selection for other purposes than avoiding the birth of a child with a serious genetic disease, we are stepping on a slippery slope towards nonmedical sex selection. Slippery slope arguments claim that we should refrain from acts or policies that, although morally acceptable in themselves, would take us down a slipway towards acts and policies that would be morally unacceptable. There are two versions of this type of argument, empirical and logical (Spielthenner, 2009). The empirical version claims that accepting position A will, as a matter of fact, make us eventually accept position $B$ as well (now regarded as unacceptable). The logical version consists of the claim that by accepting position $A$ we are logically committed to also accept position $B$. With regard to sex selection as a means to avoid reproductive burdens and transgenerational health risks, one could either argue that once we start loosening up the medical model, we will end up accepting clear cases of nonmedical sex selection as well, or that we are logically committed to doing so once we accept sex selection for other reasons than avoiding serious disease in the child to be conceived.

As in all slippery slope arguments, the primary question is whether the imagined position at the bottom end of the slope is indeed morally objectionable. Athough sex selection for nonmedical reasons does indeed raise moral concerns, it is untenable to conclude that it would necessarily lead to unacceptable social consequences (Warren, 1985). Moreover, even if one would reject all instances of sex selection for nonmedical reasons, the supposed inevitability of sliding down from accepting intermediate cases to accepting or having to accept nonmedical sex selection is far from self-evident. It is important to note that sex selection as discussed in this chapter would still be for health-related reasons. Why would accepting these lead to 
also accepting the whole range of possible motives for nonmedical sex selection? In its empirical version, the argument is highly speculative and therefore noncompelling. The logical version can simply be refuted by pointing to the compatibility of accepting sex selection for the intermediate purposes discussed in this chapter with rejecting sex selection for all kinds of non-health related reasons. This leads us to conclude that the notion of a slippery slope towards nonmedical sex selection is not a convincing argument against additional sex selection in the context of PGD or NT for mtDNA disease.

\subsection{Proportionality}

A further objection to be considered is that the (material and immaterial) costs of sex selection to avoid reproductive burdens and possible transgenerational health risks are not in proportion to the expected benefits. As the benefits are uncertain, the costs must indeed be low to justify sex selection. These costs depend on the chosen procedure. Below, we will discuss two strategies of sex selection and scrutinize whether it is morally acceptable to add (one of) these to PGD and/or NT for mtDNA disease, and if so which route is morally preferable.

\subsection{Postconceptional sex selection}

Postconceptional sex selection, i.e. sex selection of IVF-embryos, requires PGD followed by selective transfer of a male embryo. To assess the proportionality of adding sex selection at this stage, at least two (related) factors should be taken into consideration.

A first factor is whether and how large an extra step is needed for sex determination (ASRM, 1999). (1) No extra step would be needed if information about the sex of the foetus is available as a by-product of PGD already performed in the context of preventing the transmission of mtDNA disease. (2) If not, sex determination may be added to PGD (i.e. included in the initial assay), supposing again that PGD would already be performed in the context of preventing the transmission of mtDNA disease. Whether this would involve no more than a small extra step also depends on whether this would require a further biopsy of an additional blastomere. (3) If PGD is not already performed (neither as the central approach nor as a back-up after NT), a complete IVF/PGD procedure would have to be done in order to be able to determine the sex of the embryos.

A second factor refers to how this information is subsequently used. One could aim to maximize the transgenerational benefits of adding sex selection to PGD or NT for mtDNA disorders by discarding all female embryos, also if they are healthy and no suitable (healthy and (morphologically) good quality) male embryos are available for transfer. An alternative conditional approach would allow healthy and good quality 
female embryos to be transferred, but only if no suitable male embryos are available. This is similar to an earlier proposal for conditional sex selection in the context of PGD for X-linked disorders, according to which carriers are only to be transferred when non-carriers are not available (De Wert, 2005).

\section{Should postconceptional sex selection be added to PGD for mtDNA disorders?}

If the woman undergoes IVF/PGD for mtDNA disorders anyway, information about sex may automatically become available (situation 1, as described above) or be obtained without significant material or immaterial extra costs (situation 2). Situation 3 is only relevant in the context of NT, where PGD is not necessarily part of the procedure (see below). With regard to situations in which PGD is the central technology, the necessary first step - sex determination - would not seem to be so costly as to render sex selection disproportional in advance. There is one qualification to this conclusion. Things may be more complicated if, in situation 2, a further biopsy would be needed, involving a small extra risk of embryo loss and theoretical extra health risks for the child to be. Whether the limited benefits to be expected from sex selection would outweigh these extra risks is indeed debatable.

The next issue regards the proportionality of using this information in the context of selecting embryos for transfer. The maximizing approach to postconceptional sex selection implies that, generally, $50 \%$ of the embryos (all female embryos) will be discarded. This is in addition to male or female embryos that are not suitable for transfer, either because of a mutant load above the threshold for disease expression, or because of low morphological quality (Bredenoord et al, 2009). By adding embryonic sex as a further criterion of the same importance as mutant load and quality, the maximizing approach will make it almost impossible to find an embryo that would be eligible for transfer.

This higher embryo-loss can be regarded as problematic from two perspectives: the moral status of the human embryo and the general cost-effectiveness of PGD for mtDNA diseases. As we ascribe low independent moral value to preimplantation embryos, the former argument is not decisive. Other things equal, the transgenerational benefits of sex selection may well outweigh the greater loss of (healthy) human embryos. However, this is to neglect the impact of embryo-loss on cost-effectiveness, where costs refer to material costs as well as to the burdens and risks for the women involved. If female embryos are always to be discarded, this may either lead to transferring male embryos of lesser quality or to starting a new treatment cycle more often than would otherwise be the case. This would negatively affect the take-home-baby rate, expose women to greater burdens and risks and lead to a lower general cost-effectiveness. As the benefits of transgenerational sex 
selection are uncertain and probably small at best, maximizing those benefits at the cost of these effects would clearly be disproportional.

The alternative conditional approach also involves the introduction of embryonic sex as a third selection criterion, but without making this equivalent to mutant load and quality. In this approach the selection process is in two steps. The first step is to select male or female embryos that carry a mutant load below the threshold to disease expression and are of good morphological quality. If this does not lead to sufficient male embryos suitable for transfer, suitable female embryos may still be transferred. Only if suitable embryos of both sexes are available, male embryos are to be selected. This avoids the negative effects of the maximizing approach on the cost-effectiveness of PGD for mtDNA disease, but does so at the cost of losing some of the transgenerational benefits that sex selection may add. As this entails even smaller benefits, the proportionality of the conditional approach hinges on whether the remaining benefits are still important enough to justify any extra steps that may be needed for sex determination not necessarily followed by sex selection. As long as these steps are small and do not involve more than minimal extra costs and risks, we are inclined to a positive answer. Provided the couple gave consent, the preferential transfer of male embryos in a conditional two-step approach would in our view be a morally acceptable way of reducing (rather than avoiding) reproductive burdens and transgenerational risks in their offspring.

\section{Should postconceptional sex selection be added to NT for mtDNA disorders?}

Again, the first relevant question is how embryonic sex is determined. Here also, this depends on whether PGD is done anyway. That might be the case if PGD is considered necessary as a confirmatory test after NT. This would be to ensure that the carryover (of affected mitochondria coming along with the oocyte, the pronuclei or the nucleus of the recipient woman) does not result in an unacceptable high mutant load and/or to ensure that all nuclear material has been removed from the cytoplasm of the enucleated donated oocyte (Poulton et al, 2009). However, whether such a confirmatory PGD would be feasible and useful is debatable, as many questions still are unanswered. For example, if the carryover results in the presence of pathogenic mitochondria, will these segregate evenly over the blastomeres of the embryo?

Let us, for the sake of argument, first assume that PGD would indeed be useful as a back-up test after NT. In that case, the reasoning would be largely similar to that already provided above with regard to adding postconceptional sex selection to PGD. The single difference is that with regard to NT, the disproportionality of restarting the whole procedure is enhanced by the necessary involvement of an oocyte donor, who 
would then also have to re-donate. This only strengthens our conclusion that a conditional 'two-step' procedure is the only morally acceptable approach to postconceptional sex selection for the purposes discussed in this paper. With regard to applying this after NT, an important caveat is that this approach would only be feasible if NT involves the reconstruction of several embryos at a time.

In the alternative scenario, PGD is considered unfeasible as a back-up test after NT. In that case, PGD would be added to NT solely for sex determination. Since this involves considerable additional costs, it is highly questionable whether these could be justified by the limited benefits that sex selection would add. Moreover, there is the further problem of whether a biopsy especially for this purpose can be justified, given additional risks of embryo loss (which may be larger after NT as the embryo is already manipulated) and also in light of possible health risks that this may theoretically entail for the future child. Because of the theoretical nature of the latter risks, PGD is considered acceptable if the procedure can be expected to lead to important health benefits. But precisely that is less clear where PGD would only be needed to add sex selection to NT. Part of this equation should also be that in the proposed 'two-step' approach sex determination is not necessarily followed by sex selection, thus further undermining the proportionality of adding PGD for this purpose. It is difficult to imagine that PGD, conducted solely for sex selection, could indeed be added to NT in a morally acceptable way.

\subsection{Preconceptional sex selection}

Alternatively, sex selection could be performed prior to fertilization. Preconceptional sex selection requires separation of X-bearing and Y-bearing spermatozoa, with subsequent selection for artificial insemination or IVF (ASRM, 1999). Several methods have been proposed, such as the use of albumin gradients. Although a recent case study reported the birth of a boy after sex selection by albumin gradients (Chen et al, 2008), the dominant view is that this method is not reliable for clinical use (Aleahmad et al, 2009).

The most promising preconceptional technique, already used in clinical practice, is sperm sorting by flow cytometry. Recent studies seem to indicate that this method may be conducted safely and relatively effectively (Schulman and Karabinus, 2005; Karabinus, 2009), but clinical follow up is morally imperative to establish the long term safety as well. In comparison with postconceptional sex selection, preconceptional methods have the important benefit of not involving the discarding of embryos. In theory, preconceptional sex selection would allow maximizing the transgenerational benefits of producing boys without undermining the effectiveness of PGD or NT for mtDNA disease. In practice, it is important to note that even what is 
currently the most promising method for preconceptional sex selection is not fail-safe. Particularly with regard to the selection of Y-bearing sperm, the effectiveness of the procedure does not far exceed the range of $80 \%$ (Karabinus, 2009). This may be regarded as important enough, as it would at least considerably increase the chances of obtaining male embryos. And if an $80 \%$ result would not be seen as preempting the need for postconceptional sex selection, it would certainly provide a much better starting position for attempts at selecting male embryos without compromising the effectiveness of PGD or NT for mtDNA disease.

Other benefits are that preconceptional sex selection is less intrusive and costly than IVF/PGD, assuming that the latter procedures would be specifically done for sex determination and selection. This, of course, will not be the case if PGD is already being done in the context of avoiding the transmission of mtDNA disease.

\section{Should preconceptional sex selection be added to PGD for mtDNA disorders?}

If the woman undergoes IVF/PGD for mtDNA disorders anyway, a simple and cheap method of sex determination is already available. But as the subsequent step of sex selection cannot be fully deployed without undermining the effectiveness of the procedures aimed at helping the couple have a healthy child, there might still be a case for preconceptional sex selection. At $80 \%$ selection of $Y$-bearing sperm, this may be regarded as an alternative approach that would be better able to achieve the transgenerational benefits that sex selection is expected to bring than the 'conditional' postconceptional approach. Alternatively, it may be regarded as a preselection step in a combined pre- and postconceptional approach which would come even closer to maximizing transgenerational benefits of sex selection. As neither of these options would negatively interfere with regular embryo selection, the proportionality of adding preconceptional sex selection (instead of or in combination with postconceptional sex selection) would depend on the extra costs this would entail. As long as these are expected to be considerable, they may well outweigh the limited benefits that can at best be achieved by adding sex selection to PGD or NT for mtDNA disorders.

Should preconceptional sex selection be added to NT for mtDNA disorders?

In cases where after NT for mtDNA disorders, PGD would be performed as a confirmatory step, the arguments for and against adding preconceptional sex selection would be essentially the same as just given. Preconceptional sex selection may be particularly interesting in those cases where after NT, the patient is not already having PGD. In those cases, preconceptional sex selection prior to IVF/NT (or NT/IVF, depending on whether the transplantation occurs before or after fertilization) would be the least costly method, leading at least to considerably 
increasing the chances of being able to transfer a suitable male embryo. In theory, combining this with adding PGD solely for conditional postconceptional sex selection after NT would allow improving on this. Apart from the disproportionally high extra costs that this would entail, it is questionable whether performing a biopsy especially for this purpose could be justified by the small extra benefits this might yield.

\begin{tabular}{|c|c|}
\hline \multicolumn{2}{|c|}{$\begin{array}{l}\text { Postconceptional versus preconceptional sex selection: } \\
\qquad \text { a comparison }\end{array}$} \\
\hline Postconceptional sex selection & Preconceptional sex selection \\
\hline $\begin{array}{l}\text { Disproportional to start } \\
\text { IVF/PGD solely to avoid } \\
\text { transgenerational risk } \\
\text { - } \quad \text { Meaningful method if the couple } \\
\text { needs PGD anyway } \\
\text { 'Two-step' approach }\end{array}$ & $\begin{array}{l}\text { - In theory morally superior option: } \\
\text { less intrusive, less costly, no } \\
\text { embryo loss } \\
\text { - In practice: not fail-safe } \\
\text { - } \quad \text { Particularly interesting if the } \\
\text { couple does not need PGD }\end{array}$ \\
\hline
\end{tabular}

\subsection{Conclusion}

Even if the use of PGD or NT to prevent the vertical transmission of mtDNA disorders leads to a healthy child, there is the possibility that a latent mutant load will rise again above the level of disease expression in a third generation. Given the maternal inheritance of mtDNA disease, this also depends on whether the child to be conceived is a girl and whether she will decide to have children herself. Since most women want to reproduce, helping the present couple to have a boy could be a meaningful way to avoid the transgenerational health risks and related reproductive burdens that might otherwise result from a clinical application of PGD or NT for mtDNA disorders. We have argued that adding sex selection for this purpose is morally acceptable in principle. Even though the health of the child to be conceived is not at stake (as required in the tradional justification of sex selection for medical reasons), the reason for choosing or preferring a boy is still health-related. The moral concerns associated with sex selection for nonmedical reasons do therefore not apply.

Sex selection can either be done post- or preconceptionally. We have considered the proportionality of adding these different methods to PGD or NT for mtDNA disorders. With regard to postconceptional sex selection, we have concluded that the only approach that may be acceptable is one in which sex selection only comes in after 
suitable embryos for transfer are already selected on the basis of health and morphological quality. A further requirement is that the steps needed for the preceding sex determination are small and do not involve more than minimal extra costs and risks. This would only leave room for postconceptional sex selection in cases where the woman is already having PGD, and do so at the price of giving up on part of the possible benefits of adding sex selection. Saying this, we are aware that the pressure already exerted on the embryo-selection process by conventional criteria (health, quality) is high, and that the notion of there being much of a choice left at the second stage of the conditional approach may well be quite theoretical. And the more it is, the further this undermines the proportionality of any extra steps needed for sex determination.

None of these problems arise with preconceptional sex selection. Whereas this would allow maximizing the benefits of adding sex selection to PGD or NT for mtDNA disorders, extra costs are the single proportionality-limiting factor. Currently, there are no fail-safe methods of preconceptional sex selection. For selection of Y-bearing sperm, the maximum yield of the most promising method is not much higher than $80 \%$. This may be considered important enough as it would at least considerably increase the chances of obtaining male embryos, thereby also providing a much better starting position for conditional (two-step) sex selection in the context of PGD. Given remaining uncertainties about the long-term safety of sperm sorting by flow cytometry, follow-up of any children born after adding this element to the procedure would be morally imperative, as it is for NT itself.

In sum: although adding sex selection to avoid the transgenerational health risks and related reproductive burdens that might otherwise result from a clinical application of PGD or NT for mtDNA disorders is morally recommendable in principle, there are important hurdles affecting the proportionality of this idea and therefore also the moral acceptability of putting it in practice. The only cases where this does not apply are the (sparse) situations in which after PGD for mtDNA disorders 1) no extra steps are needed for sex determination and 2) sufficient healthy and good quality embryos are available for transfer. In those situations we recommend to actively select male embryos. With regard to the possible clinical application of NT, these situations may only occur if PGD already serves as a back-up. If not, sex selection in the context of NT (for the purpose under consideration) will be a morally defendable proposition only if effective and safe methods for preconceptional sex selection become available against limited costs. Of course, this will then also be relevant for sex selection in the context of PGD, at least with a view of increasing the chances of selecting suitable male embryos through preselection of Y-bearing sperm. 

Chapter 9

General Discussion 



\subsection{Introduction}

Whereas assisted reproductive technology (ART) was initially developed and used for infertility problems, it has increasingly been deployed as a vehicle for genetic technology. In this thesis I discussed and analyzed the ethical issues that arise when ART is used with the aim of preventing the transmission of mitochondrial disorders, particularly those caused by a mutation in the mitochondrial DNA (mtDNA). As no curative treatment exist for these clinically heterogeneous, but usually severe disorders, avoiding the transmission of an mtDNA mutation from mother to child is considered to be of key importance by both parents-to-be and professionals. In this thesis I aimed to ethically evaluate four possible reproductive options for carriers of mtDNA mutations: two types of genetic selection, including prenatal diagnosis (PND) and preimplantation genetic diagnosis (PGD) and two types of genetic intervention or modification, including ooplasmic transfer (OT) and nuclear transfer (NT). Subgoals of this thesis are to make an inventory of the ethical issues involved in these various reproductive strategies and, where possible and appropriate, to develop ethical guidelines for their preclinical development and clinical use.

Whereas all options have been discussed, special attention has been given to PGD and NT for mtDNA disease. At the onset of the project resulting in this thesis, it became increasingly clear that OT is not feasible as a strategy to prevent the transmission of mtDNA disorders. It is also acknowledged that PND for mtDNA mutations has its limitations and is not always considered a satisfactory method to prevent the transmission of mtDNA disease. Therefore, researchers and clinicians have been searching for alternatives. Particularly PGD and NT emerged as conspicuous potential reproductive options for carriers of mtDNA mutations. That was the main reason for focussing on these reproductive strategies instead of OT and PND. The fact that both PGD and NT for mtDNA disorders are still in its infancy provided me with the opportunity to pro-actively examine the ethics of these emerging technologies, bypassing the reproach that 'ethics is always too late'.

As it is currently accepted that OT is not feasible as a strategy to prevent the transmission of mtDNA disorders, less attention has been paid to OT. In the initial applications of OT as a fertility technique for women experiencing repeated embryonic development failure, only small amounts of donor ooplasm (10-15\%) were transferred to the recipient's oocyte. To prevent the transmission of mtDNA disease, however, a much larger amount of donor ooplasm would be needed. It is seriously questioned whether it is possible to introduce such an amount of donor ooplasm into the oocyte. As OT seems unsuitable as a reproductive option for carriers of mtDNA mutations, clinically offering OT would be both futile and unethical. Moreover, OT is 
also not proven safe as an assisted reproductive technology in general, which was recently reconfirmed by Liang and colleagues (2009). This implies that OT should not only be dismissed as a reproductive option for carriers of mtDNA mutations, but also that clinically offering OT as a fertility technique would be premature.

Below, I will present the main conclusions of this thesis and identify questions that are of broader (future) interest to ethics at the interface of reproductive medicine and genetic technology. First, I shortly rehearse the classification we used in order to make the vast and variable amount of mtDNA mutations 'doable'. Then the main conclusions of the empirical study and topics for further empirical research are presented. Subsequently, I review the main conclusions of PND, PGD and NT for mtDNA disorders, elaborate issues that deserve further attention and identify questions for future ethics research. I finalize this thesis with the question whether there is a 'moral hierarchy' in the use of the reproductive options as discussed in this thesis.

\subsection{A further classification}

The terms 'mitochondrial disorders', 'mitochondrial DNA disorders' and 'mitochondrial mutations' are sometimes interchangeably used. Strictly speaking, however, these are separate categories, raising separate ethical questions. In chapter 2, we therefore first made a further classification of the different concepts.

\section{Mitochondrial disorders and mitochondrial DNA disorders}

An ethically relevant distinction exists between reproductive options for carriers of mitochondrial disease cause by a defect in the nuclear genome and for carriers of mtDNA mutations.

The first group of mutations shows a Mendelian pattern of autosomal recessive, dominant or X-linked inheritance. Offering ART, then, is comparable with 'standard' applications of ART. This is not to say that these applications are ethically unproblematic, but they do not raise novel ethical questions in addition to the ethical discussion regarding PND and PGD in general, such as discussions regarding the moral and ontological status of the embryo, the welfare of children conceived by means of ART, whether it is morally acceptable to terminate a pregnancy on genetic grounds, whether and how non-directive counselling should take place, whether and to what extent it is possible to obtain a truly informed consent and how the fertility specialist should take into account her dual responsibility towards the couple and the future child.

When applying ART for the second group of mitochondrial disorders, the 'mitochondrial DNA disorders', however, the ethical issues are intensified, sui generis 
and sometimes even still rather unexplored. This is due to the characteristics of mitochondrial genetics, particularly heteroplasmy. This means that there is a mixture of normal and mutant mtDNA in one person, the level of which can differ among tissues. If the mutant load - the ratio of mutant to normal mtDNA - exceeds a specific threshold, clinical features become manifest. However, the exact threshold to disease expression is often not known and genotype-phenotype correlations are often lacking, even within families. In view of the above, this thesis has focussed on the ethical questions raised by reproductive options for carriers of mtDNA mutations.

\section{Mitochondrial DNA mutations}

For the latter category, the term 'mtDNA disorders' is often used, which may suggest the existence of a uniform category of disorders. In reality, there is not a 'single hot spot', but a wide array of mtDNA mutations, causing a wide array of phenotypes. In order to make a more precise inventory of the ethical issues involved, we made a further classification of the different types of mtDNA mutations.

The first category regards the de novo mutations. For these mutations, future parents have a low recurrence risk of having an(other) affected child. The second category regards the stable mutations with a predictable outcome. When the prenatal or preimplantation test shows skewed results (i.e. a very high or a very low mutant load), reliable predictions regarding the health of the future child can be made. The interpretation of intermediate mutant loads, however, is problematic. The third category regards the unstable mutations with an unpredictable outcome. For those mutations, making reliable predictions regarding the health of the future child is very troublesome. The fourth category regards the mutations with an unknown outcome. These usually include private or family specific mutations for which insufficient information is available to determine whether these mutations allow reliable predictions. The fifth category regards the homoplasmic mutations. Homoplasmic mutations are present in $100 \%$ of the mtDNA, which implies that prenatal or preimplantation testing to determine the mutant load is useless. However, as some mutations show an unequal sex incidence, prenatal and preimplantation testing could be considered for sex selection (see below).

\subsection{Empirical studies: attitudes and experiences of professionals}

Whereas a scientific and scholarly ethical debate about the possible reproductive options for carriers of mtDNA mutations is developing, not much information regarding the experiences of people directly involved with mitochondrial genetics was at hand. What are their concerns, worries and points of view? What do they consider to be the major ethical issues? 
In order to seek answers for these questions, we set up an empirical study using qualitative interview design. People that are involved in their personal life with mitochondrial genetics, i.e. patients suffering mtDNA disease or carriers at risk of transmitting an mtDNA mutation to their offspring, and people that are professionally involved with mitochondrial genetics, i.e. clinicians, researchers and policy-makers, were selected for interviews. Although I only dedicated a separate chapter to the experiences of (medical) professionals with reproductive genetic counseling in the context of mtDNA disease, all interviews served as valuable input for this thesis.

The interviews with patients / couples with a wish for a child resulted in a varied picture. Couples who were already confronted with an mtDNA disease, for example when a deceased child has crudely confronted them with the existence of an mtDNA mutation in their family, felt as 'hands-on experts' better equipped to make reproductive decisions than completely 'new' patients. In contrast to the 'technical' approach of professionals, none of the couples were interested in knowing the exact recurrence risks. They were for example more concerned about their responsibilities towards their possible children, and whether you should have children if you know about the existence of a genetic mutation beforehand. As the amount of interviews turned out to be insufficient for meeting methodologically sound saturation levels, it is difficult to draw firm conclusions. Therefore, future research on the experiences of patients / couples with a wish for a child would be valuable for adjusting reproductive genetic counseling to their preferences.

Chapter 5 reports the interviews with researchers and clinicians. Our results show how professionals struggle with the handling of ambiguous data often linked to PND and PGD for mtDNA disorders. All professionals feel that the inherent challenges of reproductive genetic testing and counseling are intensified in the context of mtDNA disorders. Professionals tend to regard the uncertainties as conflicting with their task of providing accurate information when counseling couples. They struggle with the tension between on the one hand the ideal of reproductive autonomy and on the other hand the reality of complex clinical decision-making. Whereas part of the professionals hold the opinion that reproductive autonomy is not endangered, others doubt whether the nuances of mitochondrial genetics can still be explained. If professionals cannot make a decision, how on earth could the others?

Part of the professionals has therefore decided not to offer specific types of prenatal tests or they exclude testing for some mutations (for example the unstable mutations, with an unpredictable outcome). Those more positive towards testing introduce several mechanisms to control and reduce the uncertainties. They for example emphasize that only very experienced and well-equipped professionals in leading 
centers should offer this type of testing. Furthermore, those professionals are very anxious to find out the precise risks and to communicate these to their patients.

Although knowing the magnitude of any remaining risks is necessary for determining whether testing can be justifiably offered and although it is interesting from a scientific point of view, more precise calculations will not always provide couples involved with a better basis for decision-making. Besides the fact that people have a poor understanding of quantitative risk information, it can be questioned whether knowing the risks in detail will be helpful for them (see chapter 5). This is not only of relevance in the context of mitochondrial genetics. It is a point of concern in view of the growing expansion of genetic risk information in both clinical research and clinical practice. Furthermore, although professionals' feelings of discomfort are partly inherently linked to the characteristics of mitochondrial genetics, it may also be linked to the inherent limitations in genetic knowledge representing the state of the art. After all, novel developments in medical genetics will continuously confront professionals with ambiguous information.

Next to the above mentioned further empirical studies on the views and experiences of patients and couples with a wish for a child, an interesting area for further (empirical) research would be to examine how the growing use of genetic risk information can be incorporated in clinical research and practice in a morally acceptable way, so that both patients and professionals find their way in dealing with risk information.

\subsection{Prenatal diagnosis for mtDNA disorders}

When performing PND for mtDNA mutations, making reliable predictions regarding the health of the future child may be troublesome. As a consequence, one of the main ethical concerns regards the moral acceptability of terminating a pregnancy based on ambiguous and uncertain data. Is it morally acceptable to offer PND when the test result may be difficult to interpret? How to obtain informed consent - is it possible to explain the drawbacks of testing? What about the psychological and moral consequences of terminating a pregnancy based on ambivalent data? I reviewed these and other questions in chapter 2.

For the first category of mutations, the de novo mutations, I provisionally conclude that PND is morally acceptable, and perhaps even a better route than PGD. After all, one could question the proportionality of starting an IVF/PGD trajectory for a $4 \%$ risk of having an affected child. Moreover, the chance of becoming pregnant is higher 
with natural reproduction and the risk of an affected child, and subsequently of considering a termination of pregnancy, is small.

PND could be morally acceptable for the second category of mutations as well, the stable mutations with a predictable outcome. Nevertheless, the interpretation of results in the so-called grey zone (i.e. intermediate mutant loads) remains highly problematic. As a consequence, the couple may be confronted with difficult decisions regarding whether or not to terminate the pregnancy. Whereas considering selective abortion is always difficult, this is further complicated by the uncertainty of the test results.

PND for the third category of mutations, the unstable mutations with an unpredictable outcome, is ethically very challenging and troublesome for both the couple and professionals. After all, both the probability of the disease to occur and the severity of the disease may be difficult to predict. As PGD provides the possibility of selecting between several embryos, which increases the chance of having a mildly affected or unaffected child, and as PGD avoids the difficult decision about whether or not to terminate the pregnancy, PGD may, others things being equal, be a morally favorable route for carriers of these types of mutations.

It is difficult to make a general judgment regarding the ethical acceptability of PND for the fourth category of mutations, the (usually private or family specific) mutations with an unknown outcome. Often, insufficient information is available to determine whether these mutations allow reliable predictions. The acceptability also depends on the extent in which information can be obtained regarding the predictive value of testing.

The acceptability of PND for the fifth category of homoplasmic mutations does not depend on the reliability of testing, but on how one morally evaluates sex selective abortion. As homoplasmic mutations are present in $100 \%$ of the mtDNA, prenatal testing to determine the mutant load is useless. In some cases however, PND could be considered for sex selection. In case of Leber Hereditary Optic Neuropathy (LHON), for example, males have 50\% lifetime risk of blindness compared with $10 \%$ for females. Would it be morally acceptable to terminate the pregnancy in case of a male fetus? Although this is a question for future research, it is important to keep in mind that also other methods of sex selection exist, such as preconceptional sperm sorting by means of flow cytometry and postconceptional sex selection by means of IVF/PGD followed by selective transfer of a female embryo - these methods of sex selection are described in another context in chapter 8. With these latter methods, the selection would not occur when a pregnancy is already established, but in earlier stages of development. In several places in this thesis, I deployed a gradualist position regarding the status of the embryo, containing the view that the moral status of the embryo / fetus grows throughout pregnancy - which is in line with the dominant 
view that a 35-week old fetus has a totally different moral status than an 8-cell embryo in a petri dish. In line with this position, preconception or postconception sex selection before implantation should be morally preferred over sex selective abortion.

I conclude that PND could potentially serve as an acceptable option for couples at risk of transmitting an mtDNA mutation to their future offspring. Just as we have developed moral guidance for clinically introducing PGD for mtDNA disorders (chapter 4), a task for future research should be to also develop moral guidance for PND - particularly for the unstable mutations and the private, family-specific mutations.

\subsection{Preimplantation genetic diagnosis for mtDNA disorders}

PGD was initially developed to transfer embryos without the mutation in question. As described in chapter 3 , the goal of PGD is usually defined as allowing couples at risk to have a child not affected by the particular disease. PGD for mtDNA disorders is a new and controversial use of the technology. Traditional applications are targeted at Mendelian and chromosomal disorders. In those cases, it is in principle certain whether the relevant gene defect is present and which of the available embryos can safely be transferred. In case of PGD for mtDNA disorders, this is less clear. It is conceivable that in some cycles only affected embryos are available for transfer. In those cases, it is not the goal to transfer unaffected embryos but to reduce a couples' risk of having a severely affected child. As shown in this thesis, such a use of PGD raises several ethical questions. Is it morally acceptable to start an IVF/PGD procedure knowing that there may only be affected embryos? What about knowingly bringing a child into the world whose health may be (significantly) impaired? Is PGD acceptable as a means of reducing rather than eliminating genetic risk? If so, what amount of residual risk should we still consider acceptable? To what extent should one search for 'the best possible embryo'?

We met these and other questions when examining the ethics of PGD for mtDNA disorders. The moral evaluation has been undertaken in two steps. In the first step, we scrutinized the more fundamental question of whether PGD for mtDNA mutations would be morally acceptable as such. Instead of 'promising' parents a healthy child, PGD for mtDNA disorders may sometimes only reduce reproductive risk. There are many possible arguments against such a 'risk-reducing' PGD. For example, one could argue that the transfer of an embryo at risk is at odds with the welfare of the future child. We defended the reasonable welfare standard: (medically assisted) reproduction is morally acceptable as long as the resulting child has a reasonable chance of an acceptable quality of life; a high risk of serious harm would be the 
contra-indication. From this perspective, the transfer of an embryo at risk may be justifiable as long as the child will not run a high risk of serious harm. Another possible objection to PGD for mtDNA disorders is that such an application of PGD is too complex for parents too decide - and also our empirical studies show that many professionals consider mitochondrial genetics a 'test case for reproductive autonomy'. Nevertheless, to deprive competent couples from reproductive options because the decisions are too complex amounts to a form of paternalism that would be hard to justify. Offering (PND and) PGD in the context of mtDNA disease should therefore be accompanied by adequate counseling and sufficient time. A final example regards the objection that PGD for mtDNA disorders sets us on a slippery slope. There are several slippery slopes imaginable. For example, will the acceptance of risk reduction as an end of PGD set us on a slippery slope towards accepting increasingly lower percentages of risk reduction? Or will accepting PGD for disorders with a lower penetrance and a variable expression lead us to accept smaller and smaller health problems as a reason for genetic testing? In the end, so we argued, these are questions of proportionality, to be judged on a case-by-case basis. Having scrutinized the possible objections, we concluded that none of these provide an overriding argument to dismiss PGD for mtDNA disorders beforehand. Therefore, our second step was to elaborate which conditions should be met in order to justifiably introduce PGD in clinical practice - and this is where the really complex questions come in.

\subsubsection{Two starting points}

In chapter 4, we examined the questions that may successively arise when PGD for an mtDNA mutation is considered for application in clinical practice. We defended two starting points.

The first starting point regards the reasonable welfare standard (the child conceived by means of PGD for mtDNA disorders should have a reasonable chance of an acceptable quality of life). Using this standard has several implications. It for example implies that before PGD can be considered, it should be clear whether PGD is sufficiently reliable. It is important to ascertain that the cell taken for analysis adequately represent the composition of the embryo as a whole. This issue of representativity is of utmost importance from a moral point of view: would there not be a uniform distribution, then PGD is not reliable and may result in a severely affected child - which is at odds with the reasonable welfare standard. It also implies that an appropriate cut-off point is determined. A cut-off point is the threshold of mutant load above which no embryos are considered for transfer - and below which embryos are eligible for transfer. 
By avoiding a high risk of serious harm, the reasonable welfare standard formulates the lower limit of medical acting - corresponding to the principle of nonmaleficence (do not harm). If however, other things being equal, it is reasonably possible to reduce the risks of a medical treatment (or to enhance its benefits), ART included, then that is of course what the medical team should aim at - corresponding to the principle of beneficence. This is the rationale of the second starting point: continuous efforts should be made to minimize the risks as much as reasonably possible. This implies that although residual risks may well be acceptable, the parents and the medical team should make efforts to search for the embryo with the lowest mutant load. This may for example be done by generating more embryos. After all, the more embryos available, the higher the chances of obtaining no or low mutant load embryos. I discussed several possibilities for searching for 'better' embryos, such as starting a new IVF/PGD cycle. Although it is morally acceptable that a couple and the medical team confer about whether or not to start a new cycle, looking for better embryos should, however, not be seen as morally required if an embryo below the cut-off point is available - particularly because of the risks and burdens of an IVF/PGD cycle.

An interesting question for future research would be to examine how this moral obligation to reduce the risks as much as reasonably possible relates to the acceptability of certain forms of so-called coercive offers: if a couple asks for treatment $A$, then the physician could decide to only offer this treatment $A$ in combination with treatment $B$. Treatment $B$ is thus imposed as a condition for access to treatment $A$. In this thesis, there are many situations wherein such a coercive offer came up. For some mtDNA mutations, for instance, it is not exactly known whether the mutant load determined in the embryonic phase is a reliable predictor for the health of the child. Furthermore, the mutant load may change over time. Is it morally acceptable that the medical team imposes PND after PGD (or, possibly in the future, after NT), as a confirmatory back-up test? Or, to put it differently, is it morally acceptable that the medical team only gives access to PGD on the condition that the woman's intention is - if pregnant - to have a confirmatory PND and a termination of pregnancy in case of an adverse test result? Another example regards the imposition of embryo donation for research. Analysis of affected embryos may shed light on the mechanisms of mtDNA genetics and reduce the uncertainties currently linked to ART for mtDNA disease. Is it morally acceptable that the medical team only gives access to PGD to couples who agree to donate their leftover embryos for research? To elaborate these and other examples is outside the scope of this final chapter, but it would clearly be interesting for further research. 
There are three further issues raised by PGD for mtDNA mutations on which I would like to make some additional comments. Below, I will first shortly address the question to what extent we should comply with the wish to have genetically related children when other routes to have children may be safer. Second, I will make some additional comments regarding the 'reasonable welfare standard'. Third, I identify some important questions for further ethical debate in the context of medical scientific research with minors.

\subsubsection{Possible alternatives and the wish for genetically related children}

If after the IVF/PGD procedure an embryo carrying a low mutant load is transferred, the resulting child may develop clinical disease symptoms (and the same may count for a possible future application of NT for mtDNA disorders). One of the possible objections against such an application of PGD, as discussed in chapter 3 , is the argument that there are alternatives allowing the couple to have a healthy child. They could for example opt for IVF using donor oocytes or apply for adoption. We argue in chapter 3 that there are several reasons why these options would not count as real alternatives. A first reason is that the availability of donor oocytes and adoption is rather limited in reality. A second reason is that the majority of couples prefer to have a (fully) genetically related child. This led us to conclude that as long as there is no high risk of serious harm, it is up to the couple to decide how they balance the wish for a genetically related child with the possibility that this child may be affected.

This conclusion applies to the specific context of PGD for mtDNA disorders. It is questionable whether we should immediately extrapolate this to all reproductive technologies where a residual risk may occur. Is it always up to the future parents to decide how they balance the wish for a genetically related child with the possibility that this child may be affected? ART is often a risky, expensive, time-consuming and suboptimal procedure. To what extent should we accept risky ART when (much) safer routes are available as well? As these questions become more urgent in view of the development of NT for mtDNA disorders (see below), I identify some ingredients for further debate.

First, it is conceivable that we mistakenly exaggerate the value of genetic relatedness. Studies for example show that people experience social pressure, not only to have children, but also to have genetically related children (Strong, 1997). Other studies suggest that the absence of a genetic and/or gestational link between parents and their child does not have a negative impact on parent-child relationships or on the psychological well-being of mothers, fathers or children - although a caveat should be made for the fact that the children were very young at the time of this 
study; the results cannot be extrapolated to adolescents (Golombok et al, 2006). Suppose, for the sake of argument, that people are as happy with non-genetically related children as with genetically related children - or, to formulate it cautiously, that raising children without a genetic tie does not jeopardize positive family relationships or parental wellbeing. What are the implications for values such as reproductive autonomy, the welfare of the child or distributive justice? Second, although raising children without a genetic tie may result in satisfied parents, it may at the same time involve different experiences (Strong, 1997). Most of parental experiences will concur: the experience of child rearing, the creation of a person and a link to future persons will not depend on whether a child is genetically related. However, some experiences will differ. One may then for example think about sexual intimacy resulting in a baby, although this experience will be absent in case of ART as well. One could also think, in case of adoption, about the experience of pregnancy and childbirth. How should this consideration be taken into account? Third, as already mentioned above, even if a couple would be indifferent about whether or not their children are genetically related, then still ART may in reality be the best accessible route for them. Both adoption and oocyte donation are no easy shortcuts for having children. These alternatives may perhaps look attractive in theory, this is often not the case in practice.

For this moment, my conclusion is that as long as ART does not result in a high risk of serious harm for the resulting child, it is up to couples to decide how they balance genetic relatedness with other values. I take the choice about whether or not having genetically related children to be to a certain extent part of reproductive autonomy (Buchanan et al, 2000). This however is not to say that the desire for genetically related children trumps all other values, and in some cases it can be questioned whether the benefits of a specific technique resulting in a genetically related child still outweigh the risks of this technique.

\subsubsection{The welfare of the child: the reasonable welfare standard}

One of the possible objections against PGD for mtDNA disorders extensively addressed in chapter 3 regards the view that to knowingly conceive a child with a (significantly) higher risk of developing a disease is at odds with the welfare of the child. In reproductive medicine, doctors have a dual responsibility: towards the couple asking for help and towards the child conceived by means of their assistance. The dominant approach of assessing the acceptability of ART regards judging the welfare of the child conceived as a result of ART. The debate can be presented as different positions on the specter, ranging from the 'minimal threshold standard' to the 'maximum welfare standard'. Whereas the minimal threshold standard accepts 
ART as long as the future child has a life that is not so awful that it could have better been dead, the maximum welfare standard only accepts ART if it leads to a child with the best possible quality of life. In chapter 3 , we argue that none of these standards is satisfying. As already mentioned above, we defend an intermediate view: the 'reasonable welfare standard'.

A longstanding debate in philosophy regards the question whether future people can be harmed by being brought into existence. This debate has especially been influenced by what Parfit coined the 'nonidentity problem' (Parfit, 1984). Although it is beyond the scope of this final chapter to fully explore this philosophically complex and controversial issue, I would like to make some comments on the relationship between this debate and the reasonable welfare standard.

The nonidentity problem challenges many of our basic intuitions about obligations to future persons. For example, many people intuitively feel that it is wrong that a woman conceives a child using sperm of her deceased partner (post mortal insemination), or that it is wrong to use PGD when this results in a child with serious mitochondrial disease symptoms. The nonidentity problem, however, shows that only two types of acts can be harmful to the child: either bringing into existence a child that has a truly awful life, or bringing into existence a child with a life worth living while we had the alternative of bringing that same child into an existence that was substantially better (Roberts and Wasserman, 2009).

Neither applies to the examples above: although, in the first example, the resulting child would prefer to grow up with his father, he may still have a life worth living. Had the woman reproduced using sperm from a still living man, this would have resulted in another child. Similarly, although a child with mitochondrial disease symptoms will have harsh periods, she may still have a reasonable quality of life. Had the parents and the medical team decided to transfer an embryo without mutant mtDNA, this would have resulted in another child. Therefore, to transfer an embryo with mutant mtDNA would not amount to violating the interests of the child, as the only alternative to this particular child would be non-existence.

One could choose to accept the above and bite the bullet, which may imply that one accepts all applications of ART as long the child's life is expected to be not so awful that it would be better off dead (Roberts, 2009). Those adhering to the minimal threshold standard defend this view. In my opinion, however, this view is highly unsatisfying. Moreover, it would have far reaching implications for public policy and medical practice, as it would open the door for many applications of ART hitherto considered unacceptable. Although being brought into existence does not harm the 
child, this does not make it a matter of moral indifference what quality of life the child has. Precisely for this reason we defended the reasonable welfare standard: although we accept that a child cannot be harmed by being brought into existence (except when it has a life not worth living), this does not settle the issue regarding the justification of offering ART. As we conclude in chapter 3 , it is still meaningful to say that bringing a seriously handicapped or disabled child into the world is to cause avoidable suffering, falling short of parental and professional responsibilities.

As a consequence of accepting that no one can be harmed by being brought into existence, one cannot ground the reasonable welfare standard in a so-called 'personaffecting perspective'. Therefore, it should be grounded in a 'non-person affecting', or impersonal, view. From this perspective, an act may be wrong, not because it harmed a particular person, but for other reasons. One could for example argue that an act may be wrong when it contributes to overall suffering in the world. Therefore, parents and professionals have, within reasonable limits, the responsibility to prevent unnecessary suffering. One could also argue that an act may be wrong because it violates the interests of third parties, such as parents and siblings, professionals, or society. Parents may have an interest in not raising a diseased or handicapped child, for example because this would result in much stress, grieves and time-efforts. It may interfere with their plans and aims in life. Also siblings may have an interest in not being raised in a family with a diseased child. Or society may have a (financial) interest in ensuring that as few children as possible are born with disabilities (Hope and McMillan, 2003) - although this latter should never be an overriding consideration: a society actively promoting what kind of children we should have violates reproductive rights.

Some commentators have argued that the non-person affecting perspective is incoherent. For example, if one consistently denies specific applications of ART or stimulates the transfer of embryo A over embryo B with the aim to promote well-being in general, this raises 'the specter of an unconstrained obligation to procreate', as creating more persons can be a way to increase well-being (Roberts and Wasserman, 2009). However, I did not argue that people should promote well-being, but that they should not contribute to unnecessary suffering. Furthermore, I added the qualification that parents and professionals have the responsibility to prevent unnecessary suffering within reasonable limits. If parents and professionals can avoid serious suffering, limited opportunities or serious loss of happiness in future children, without substantial burdens, risks or costs, then they should do so (Buchanan et al, 2000). 
Although the non-person affecting perspective would be the most appropriate view, I do not claim that all difficulties are solved with the use of this perspective. Future research to further underpin the philosophical fundamentals of the reasonable welfare standard would be valuable.

\subsubsection{Non-therapeutic medical scientific research with minors}

A probably controversial recommendation made in chapter 4 is to at least initially genetically test the children born as a result of PGD for mtDNA disorders. The rationale would be to validate the $P G D$ procedure to ascertain that the genetic test taken in the embryonic phase accurately predicts the genotype (and phenotype) of the child. The same question will present itself in a possible future application of NT for mtDNA disorders. Also here, it would be of immediate relevance to know whether the child conceived as a result of NT carries mutant mtDNA. Not so much for the child itself (there is currently no curative treatment), but to validate the NT procedure by checking whether amounts of affected mitochondria will come along with the oocyte, pronuclei or nucleus of the recipient woman and if so, how much.

This type of genetic testing would entail non-therapeutic medical scientific research with minors (newborns). Non-therapeutic medical scientific research with minors is a contested issue, only considered acceptable when it meets some stringent conditions. Although most countries and international guidelines adhere to the socalled 'minimal risk' or 'minimal harm' standard (WMA, 2008), the precise filling-in of this standard (slightly) differs per country. In the United States, for example, the minimal risk standard allows Research Ethics Committees to approve nontherapeutic pediatric research when the risks are minimal, or a minor increase over minimal risk. Whereas 'minimal risk' is defined as the risks children face in daily life, or during the performance of routine physical or psychological examinations or tests, a 'minor increase over minimal risks' is not defined (Wendler and Emanuel, 2005; Wendler, 2009). In the Netherlands, the minimal harm standard is interpreted more stringently, only allowing pediatric research when it does not involve more than negligible risk and minimal burden to the child.

It is debatable whether genetic testing of newborns in the context of PGD or NT for mtDNA disease would meet the minimal harm standard - no matter whether interpreted widely or narrowly. We nevertheless cautiously concluded that genetic testing could be acceptable in this very particular context. This conclusion is in line with the emerging international debate on whether the minimal risk standard is still appropriate. It is increasingly recognized that too strong conditions not only protect children, but also withhold them the possible benefits of scientific research. 
Therefore, several proposals are made for modifying the current framework. Wendler (2009), for example, argues that the level of research risks to which it is appropriate to expose children depends on their maturity level. While younger children should be exposed to only very low research risks, it can be appropriate to expose older children to somewhat higher risks. In the Netherlands, a government advisory committee ('the Doek Committee') very recently concluded that at present insufficient research aimed at progress in pediatric medical science and the development of quality care for ill children is carried out (Commissie Doek, 2009). As they conclude that Dutch legislation (the Medical Research Act) forms a hinder to important types of medical research involving children, the Doek Committee makes several recommendations for cautiously widening the criteria for research with children. They for example advice to alter the current 'no, unless' principle (research with children is in principle unacceptable, unless it meets some stringent conditions) into a 'yes, if' principle (research with children is in principle acceptable).

I expect the report of the Doek Committee to cause some important policy changes in the Netherlands, also because the Dutch Central Committee on Research involving Human Subjects in practice sometimes already deployed a flexible interpretation of the minimal risk requirement, approving research that strictly speaking would involve more that minimal risks to the minor (Commissie Doek, 2009). It however remains to be seen whether these policy changes will allow genetic testing of newborns as described in chapter 4 . The Doek Committee recommends to slightly widening the criteria for interventional medical research involving children, but maintains the current framework for observational medical research with children under the age of 12. The reason is that whereas in interventional medical research there may be a chance that the child can benefit from being a study participant, this is unlikely in observational medical research. As children under the age of 12 require the greatest level of protection, the Committee recommends to maintain the current framework for judging the acceptability of observational medical research - with the caveat that to be in line with the Convention on Human Rights and Biomedicine (Council of Europe, 1997 art 17) the word negligible risk should be replaced with minimal risk. This would thus imply that the minimal risk standard would remain the framework for judging the acceptability of genetic testing of newborns. However, the Committee also acknowledges that there are differences between children and recommends to allow Research Ethics Committees to give the particular circumstances of a specific category of children more weight in their proportionality assessment. This may be of relevance for our case under study, involving a very specific type of research for a very specific group, namely children born as a result of PGD (of NT) for mtDNA disorders. 
It is clear that future research is required to discuss, firstly, the acceptability of nontherapeutic genetic testing for scientific purposes (also in the context of (competent) adults) and, secondly, the tenability of the current conditions for conducting nontherapeutic scientific research with children, and specifically newborns.

\subsection{Nuclear transfer for mtDNA disorders}

NT for mtDNA disorders is a treasury for ethicists. It concerns a technology where debates on embryo research, reproductive cloning, germ-line modification, the social meaning of novel families, oocyte donation and international regulation come together. Unfortunately, to scrutinize all these elements separately would have required another thesis. Therefore, we had to make a selection. As described in chapter 6, we first identified the ethical issues involved in NT for mtDNA mutations, both the ethical issues surrounding the development of NT in the laboratory as well as ethical issues associated with a possible clinical application. Furthermore, we distinguished the conceptual issues from the ethical issues.

We identified two conceptual issues. The first conceptual issue regards the question whether NT is a type of germ-line modification. The mitochondrial genome differs in many aspects from the nuclear genome: first, the mitochondrial genome contains only a tiny amount of the total amount of DNA; second, it has a very specific function (providing the cell's energy metabolism); third, it has its own transmission pattern (maternal and non-Mendelian). However, notwithstanding these differences between the nuclear DNA and the mtDNA, the distinctive feature of germ-line modification is that the genetic modification can be transmitted to further generations. Therefore, there is no good reason for not also applying the concept of germ-line modification to modification of the mitochondrial genome.

The second conceptual issue regards the question whether NT is a type of human cloning. This actually is a rather complex question, also depending on which definition of cloning one deploys. One variant of NT, in which the nucleus of a blastomere is transferred to an enucleated oocyte, may indeed amount to reproductive cloning. More in particular, it may amount to reproductive embryo cloning. If one deploys a 'broader' definition, then reproductive cloning refers to genetic copying - in our case the copying of an embryo. If one deploys a 'narrow' definition, then genetic copying is necessary but not sufficient: reproductive cloning also presupposes genetic identity with at least one other born individual. In that case, transferring the nucleus of a blastomere to a donor oocyte would only involve reproductive cloning as long as at least two identical embryos are thus made, transferred and carried to term. 
Next to these conceptual issues, the ethical issues associated with NT for mtDNA disease are manifold. For example, one could argue that NT leads to a child with two genetic mothers, one providing the nuclear DNA, the other the mtDNA. This provokes a discussion about the moral implication of three genetic parents and the social meaning of genetic parenthood. Other ethical questions regard the acceptability of oocyte donation, both for research and for reproductive purposes. Another major point of concern regards the health and safety risks of NT (see below).

Whereas chapter 6 identified the normative issues involved and aimed to map the moral landscape, chapter 7 deepened the ethical issues involved with germ-line modification and reproductive cloning. Although controversial, particularly when NT would imply reproductive cloning, our analysis shows that no categorical objections to germ-line modification and/or reproductive cloning exists that would provide arguments to reject NT beforehand. For example, some people have argued that germ-line modification would violate the child's right to an open future. This means a right to have one's future options kept open until one is capable of making one's own decisions. We argue that a clinical application of NT to prevent mtDNA could still be compatible with the position that one should not violate the child's right to an open future. To prevent that a child is predetermined towards a specific plan of life, it seems reasonable to only allow modification that broadens what Buchanan and colleagues (2000) have coined 'general purpose means', which are useful for nearly all plans of life. As health is a sine qua non for many plans of life, this could be considered a 'general purpose means'. The precise and further filling in of what general purpose means exactly are would be an interesting topic for further research.

Germ-line modification of the nuclear DNA is usually considered to be more controversial than modification of the mtDNA. The reason is that modification of the mtDNA would not entail the possibility to alter essential characteristics of the individual. In chapter 7 , we problematise this position. First, although it indeed is the dominant view in science that only the nuclear DNA contains the ingredients for our characteristics, this is not a fully settled issue. Second, opponents of germ-line modification often use terms such as 'essential characteristics' or 'identity determining characteristics', but they rarely define them. How could one use these concepts as important preemptive arguments without elucidating the concepts?

This led us to analyze the question whether germ-line modification indeed affects the identity of the person born. We discern two types of identity, namely qualitative identity and numerical identity, and argue that even if the mtDNA would have a basic cellular function, then it is still meaningful to say that modification of the mtDNA is 
likely to at least change the qualitative identity of the future child: the person without the mtDNA disease will have a different experience, with a different biography and perhaps even a different character than a person suffering from mitochondrial disease. This means that the dichotomy between germ-line modification of the nuclear DNA and germ-line modification of the mtDNA is untenable from this perspective.

Recently, two studies have shown that a specific variant of NT is feasible in nonhuman primates (Tachibana et al, 2009) and in human oocytes (Craven et al, 2010). Due to these technical breakthroughs, it seems only a matter of time before a first-in human clinical trial is considered. The health and safety risks of NT, however, are a major point of concern. First, it is unknown whether a mixture of mtDNA from two different origins is safe. Second, much is still unknown about epigenetic factors such as nucleo-mitochondrial interactions. Third, in a clinical application of NT it may be difficult to avoid small amounts of affected mitochondria coming along with the oocyte, the pronuclei or the nucleus of the recipient woman. Although it is unlikely that this small amount of mutant mtDNA leads to clinical disease symptoms in the resulting child, it may rise to clinically relevant amounts in a next generation. This also counts when in case of PGD a low mutant low embryo is transferred. Due to the existence of a genetic bottleneck, a low-level mutant load in the second generation (the child to be conceived through PGD or NT) may rise to higher levels in the third generation (the couple's grandchildren). As mitochondria are transferred maternally, male offspring will not pass on their mutant DNA to the next generation. This leads to the question whether the avoidance of transgenerational health risks provides a morally acceptable reason for sex selection.

In chapter 8, we examine the moral acceptability of adding postconceptional or preconceptional sex selection to PGD and/or NT for mtDNA disorders. With postconceptional sex selection we refer to sex selection of IVF-embryos, which requires PGD followed by selective transfer of a male embryo. With preconceptional sex selection we refer to sex selection prior to fertilization, which requires separation of $X$-bearing and Y-bearing spermatozoa, with subsequent selection for artificial insemination or IVF. In chapter 8 we argue that adding sex selection for this purpose is morally acceptable in principle. Even though the health of the child to be conceived is not at stake (as required in the traditional justification of sex selection for medical reasons), the reason for choosing or preferring a boy is still health-related. However, the proportionality of adding post- and/or preconceptional sex selection to PGD or NT for mtDNA disease is a relevant concern; there are important hurdles affecting the proportionality of this idea and therefore also the moral acceptability of putting it in 
practice. The only cases where this does not apply are the (sparse) situations in which after PGD for mtDNA disorders 1) no extra steps are needed for sex determination and 2) sufficient healthy and good quality embryos are available for transfer. In those situations we recommend to actively select male embryos.

The recent technical successes in NT underscore the urgent need for a further ethical debate on the moral acceptability of NT for mtDNA disorders. In this General Discussion we aim to give a further impetus for this debate by identifying two issues that ask for short-term further commentary: the issue of so-called 'three genetic parents' and the question of how new ART, among which NT, could be introduced into the clinic into a morally acceptable way.

\subsubsection{Three genetic parents}

NT for mtDNA disorders results in a child carrying the nuclear genome from her parents and the mitochondrial genome from the oocyte donor. This invokes both conceptual questions and ethical questions. Below we will highlight the main ingredients for a further debate.

Conceptually, the moot point is whether the child conceived by means of NT indeed has three genetic parents. The intentional parents will contribute the nuclear DNA, which is about $99.90 \%$ of the total amount of DNA, i.e. about 24000 nuclear genes. The oocyte donor will contribute the cytoplasm, which contains the mitochondrial genome. This is $0.1 \%$ of the total amount of DNA, namely the 37 mitochondrial genes. When the Human Fertilisation and Embryology Authority (HFEA) assigned a research license to Newcastle University to investigate the feasibility of a specific type of NT, the media announced 'babies with three parents ahead' (e.g. Barnett and McKie, 2004; Randerson, 2004; Hirschler, 2008). The novelty of NT indeed is that three persons contribute their DNA to a child (although NT shares this novelty with OT). Could we already differentiate between social, rearing, gestational and genetic parenting, should we now add the 'mitochondrial mother' to this list?

A distinction can be made between parenthood and genetic parenthood. Different accounts of parenthood exist, genetic parenthood being one of these (Kolers and Bayne, 2001; Fuscaldo, 2003; 2006; Bayne and Kolers, 2003; 2006). It grounds parenthood in direct genetic derivation. Whereas direct genetic derivation may once have been an obvious concept, new reproductive technologies urge us to specify what it exactly entails (Mertes and Pennings, 2008). After all, does passing on one's mtDNA amount to 'direct genetic derivation'? Recently, Mertes and Pennings (2008) made efforts to specify the account of genetic parenthood. One of their criteria for 
genetic parenthood is that genetic information is passed on from parent to child. The authors conclude that this criterion is not fulfilled in the context of NT to prevent mtDNA disease, as only a very limited transfer of genetic information occurs. They however do not specify how much genetic information should be passed on to the child in order to fulfill this criterion. Clearly, the mtDNA donor would only contribute a very small amount of genetic information, but it is genetic information though.

Whereas Mertes and Pennings do not ascribe genetic parenthood to the oocyte donor, Robertson (1999) does. By characterizing the provider of the nuclear DNA as the 'major genetic mother' and the provider of the mtDNA as the 'minor genetic mother', he seems to assume that the mtDNA donor indeed is a genetic parent. Clearly, there is no consensus regarding this conceptual question. This is, by the way, not unusual when searching for definitions in debates on new reproductive technologies: conceptual and ethical discussions are often intertwined. Our understanding of motherhood, fatherhood and family is normatively determined, often resulting in prescriptions disguised as descriptive definitions. In other words, a definition is often more than mere terminology (Macklin, 1991).

Even if there would be common agreement regarding a coherent definition of genetic parenthood, and whether the mtDNA donor should be included, then still this would not settle the question whether it is morally acceptable to bring a child into the world by means of NT for mtDNA disorders if it requires the involvement of the genetic material of three people. Some have argued that, because of the (unknown) psychological, legal and social consequences, this splitting of genetic motherhood should be considered the main ethical concern in the debate about NT to prevent mtDNA disease (Robertson, 1999; Szebik, 1999).

Once one makes use of a gamete donor, a third person is involved in the usually twoperson enterprise of making a baby. In case of NT, not only three persons are involved, but also their DNA - albeit a very tiny amount. What are the consequences for the welfare of the child? Does the contribution (and involvement) of DNA from three persons negatively affect the identity of the child? How should we take into account the interests of the oocyte donor? Is this type of oocyte donation morally different from other types of collaborative reproduction?

Compared to 'normal' oocyte donation, the genetic contribution in case of NT is quantitatively much smaller, because it just regards a small amount of DNA (and of course the cytoplasm). In full gamete donation, the child will inherit half of its genes from the donor. Robertson (1999) has argued that as oocyte donors normally donate both nuclear DNA and mtDNA, donation of the mtDNA (which is $0.1 \%$ of the total amount of DNA) should be morally acceptable as well. This comparison however 
does not entirely hold true, as in full gamete donation only two genetic parents exist, whereas in case of NT the child carries a mixture of three types of DNA. This may be more confusing for the child and may raise questions about kinship and identity (Robertson, 1999). The genetic contribution in case of NT is also likely to be qualitatively much smaller. If the mtDNA only has a basic cellular function, then it is out of question that the child recognizes characteristics of herself stemming from the oocyte donor. If the mtDNA does have a more significant role, then the child may indeed recognize some characteristics, or it may be confused about its identity, for example because it may feel (biologically) attached to two mothers.

In view of the current preclinical developments of NT for mtDNA disorders, the conceptual discussion regarding genetic parenthood as well as the moral discussion regarding the acceptability (and social meaning) of this type of collaborative reproduction should be addressed timely.

\subsubsection{How, if ever, could one justifiably introduce NT into the clinic?}

During the last decades, medical research involving human beings has been highly regulated and embedded in ethical procedures and standards. However, whereas review and formal oversight has been established for pharmacological products and medical treatments, this is not the case for new technology at the intersection of assisted reproductive and genetic technology. So far, many answers on safety questions in ART are determined largely by experience and the practices that evolve with the technique as it is used, using a 'try it and see approach' (Hawes et al, 2002).

Attention is now been drawn to this gap. The European Society of Human Reproduction and Embryology signalized that the widespread adoption of new techniques frequently takes place without the necessary evaluation of their efficacy, effectiveness and safety consequences (Pennings et al, 2007). Furthermore, the President's Council (2004) and the Medical Research Council (2004) concluded that professional societies and clinicians should develop a more systematic mechanism for reviewing experimental procedures before they become part of standard clinical practice. Some even argue that the lack of such a framework 'presents one of the most striking pieces of unfinished business for research ethics' (Kimmelman, 2008:240).

So, the issue is not whether guidelines for introducing new ART should be developed but what these guidelines should look like. The topicality of this issue was recently shown by two papers reporting technical successes that may soon allow clinical trials for mitochondrial gene replacement by means of (pro)nuclear transfer (Tachibana et al, 2009; Craven et al, 2010). Following the statements of Mitalipov's team (e.g. Kuehn, 2009), application for FDA approval to start the first human clinical trials will 
just be a matter of time. This gives rise to a large array of ethical questions. How to make the step from non-human primate research to the first-in-human trial? When to launch the first trial, with how many participants, and who should be eligible for inclusion? How to assess the risks and benefits, when so many factors are still unknown? I identify three issues that ask for further elaboration when considering the first-in-human trial of NT for mtDNA disorders. These three issues are far from conclusive, but they highlight which issues should at least be addressed.

A first step regards a clarification of the risks and benefits at stake. The possible benefits of NT for mtDNA disorders, the birth of a (healthy) child, seem clear. Some have argued, however, that this only counts in cases in which a child would not be brought into being in some other way (Malek, 2007). Thus, this would only count when a couple would otherwise choose not to reproduce. In view of the availability of reproductive alternatives, such as oocyte donation, PND and PGD, it is highly questionable whether this would be the case here.

What, then, about the risks of NT for mtDNA disease? When dealing with germ-line modification, uncertainty, unpredictability and irreversibility of (direct and side) effects exist (Walters and Palmer, 1997; Editorial Nature, 2008). Because of these factors, the framework for assessing the potential benefits of NT on the one hand and the health and safety risks on the other hand becomes a pivotal issue. It is at the same time also highly problematic to make a risk-benefit assessment: assessing risks (defined as the probability that harm occurs) is a difficult enterprise.

Although preclinical studies can shed important light on the risks of the technique, proof of safety of NT for mtDNA disorders can in the end only be given by doing it. Nevertheless, extensive efforts should be made in the preclinical phase to a) estimate the risks of a clinical application and b) reduce the risks as much as possible. Two types of preclinical studies can contribute to these aims, the first one being preclinical animal studies. It is reassuring in this sense that Tachibana and colleagues report the results of their experiments on nonhuman primates. Nevertheless, the use of animal models, particularly the use of primates, is an ethically contentious issue as such. Furthermore, some risks or damage of the procedure may only manifest after years. This provides an argument to wait some years with human applications of NT - perhaps even until the primates have reproduced themselves. The second type of preclinical studies regards embryo research. Part of this research may be conducted with embryos left over after IVF. Researchers at Newcastle University for example use abnormally fertilized (uni- and tripronuclear) zygotes left over after IVF for their experiments on NT for mtDNA disorders (Gardner et al, 2007; Craven et al, 2010). It may be necessary to also 
create embryos carrying an mtDNA mutation, for example for studying mtDNA segregation over the blastomeres of the embryo and for practising the technique. As discussed in chapter 4 , the creation of human embryos for research is in many countries both legally prohibited and morally rejected. The widespread prohibition of such research, however, wrongly sends the message that the moral value of early embryos outweighs the interests of prospective parents and future children (De Wert, 1999). Women and children should not be turned into trial subjects for the sake of protecting embryos - in this context, McLaren compared the transfer of embryos that have been exposed to experimental techniques with making the first test of a new aircraft-guidance system on a crowded Boeing 747 (McLaren, 1989:469; Health Council, 1998).

A second step is how to assess the risk-benefit ratio. A too pushy attitude may lead to accidents, but if one had been too cautious, many innovations (such as IVF) would have never existed. How to find an acceptable balance? A principle that has been used in decision-making in environmental and public health matters is the Precautionary Principle (PP) (Sunstein, 2002-3; Gardiner, 2006; Hughes, 2006; Kopelman et al, 2004). As some argue that the best way to see the PP is as an approach to making decisions under ignorance (Resnik, 2004) or to address uncertain risks (van Asselt and Vos, 2006), it may be a valuable principle for developing evaluation criteria to assess the risk-benefit ratio in NT. This, however, requires an examination of the definition and scope of the PP, as no less than $27($ !) versions of the PP exist. The strong version counsels that we should avoid harm until safety is established through clear evidence: 'better safe than sorry' (Sunstein, 20023) (or the Hippocratic prescription: in dubio abstine). Where some argue that taking precautions makes good science (e.g. Howard and Saunders, 1999), others argue that it stifles discovery or has paralyzing effects (Holm and Harris, 1999; Sunstein, 2002-3). A middle road is taken by those who argue that a weak or adjusted version of the PP could be valuable (Gardiner, 2006; Hughes, 2006). The basic constituents of the PP are the existence of a risk, the possibility of harm and (scientific) uncertainty concerning the actual occurrence of this harm (EGE, 2007). Clearly, key terms such as harm (e.g. what actually constitutes harm in the context of reproductive medicine, in view of Parfit's non-identity problem) need further elaboration as well.

A third step is to guarantee an adequate informed consent. While new ART widens reproductive options for couples it at the same time makes them dependent on experts - and these experts may have a double interest as a researcher and a 
clinician. It may be fruitful to explore the role, position and responsibilities of Research Ethics Committees in view of this possible tension.

If preclinical studies show promising results, then one of the most challenging questions is when it is justified to make the move from bench to bedside. When are enough safeguards made to justifiably introduce NT for mtDNA disorders into the clinic? Surprisingly, whilst this translational step is probably the most important one, it is at the same time one of the least elaborated step, with no clear guidance being available. Clearly, this sketchy overview shows that here lies an important task for further ethics research. This deserves a prominent place on the ethical agenda now that the first human applications of NT for mtDNA disorders seem imminent.

\subsection{A moral hierarchy?}

I will finalize this thesis with the question whether it is morally neutral which reproductive option a couple prefers to avoid their future child having an mtDNA disorder. One of the main conclusions is that all strategies, with the exception of ooplasmic transfer (OT), could potentially serve as acceptable reproductive options for couples at risk of transmitting an mtDNA mutation to their future offspring. I use the adjective 'potentially' to indicate that none of the strategies is unconditionally acceptable. All reproductive options ask for a further filling-in. Whereas PND and $P G D$ require further qualifications for a responsible use in clinical practice, NT requires further preclinical research before a clinical application could be appropriate.

The conclusion that all strategies could potentially serve as acceptable reproductive options gives rise to the question whether there is a 'moral hierarchy' in the use of these techniques. If several options are available, does it matter which strategy is opted for? Could one of the options be morally preferable, or is it morally indifferent which strategy is used? Does reproductive autonomy imply that couples have unqualified access to all reproductive options, or could there be reasons for restricting access? These questions are not only relevant in the context of mtDNA disorders, but raises in many other situations as well.

The key word in this discussion regards the concept of subsidiarity. This principle is particularly known as a political principle, counselling that all actions in social and political life should be performed by the lowest possible unit of government. It for example is an important principle in the European Union, where it entails that only those tasks which cannot be performed effectively on a national or local level are allowed to be performed on the central European level (Hancock et al, 2002). In the health care context, taking into account the subsidiarity of a certain treatment usually implies taking into account whether other (less intrusive) possibilities or interventions 
are available. In a clinical trail, for example, one should not include 500 research participants if 250 will do as well to answer the research question. And if the research question can be answered by means of taking a blood sample one should not take a biopsy. Translated to our discussion, the principle of subsidiarity contends that when various interventions are available to prevent the transmission of an mtDNA mutation, one should opt for the approach that offers the least possible costs. This includes the material costs, such as laboratory costs and the salaries of the personal involved, as well as the immaterial costs, such as the physical, moral and emotional burden of a certain treatment. If a strategy would provide the same outcome against lower costs, should that strategy then prevail?

Although this is an obvious question at face value, answering the question actually involves a complicated normative 'calculation'. After all, formulating which reproductive strategy can be conducted with the least possible costs requires a further filling in of what constitutes, or of what we should perceive to be the least material and immaterial costs. Is the safest strategy the least costly intervention? Or is the cheapest intervention the least costly intervention? Or perhaps the least burdensome intervention should be considered the least costly?

Peters (2004) has defended the view that a moral obligation exists to use the safest procreative method reasonably available, which particularly arises when parents and providers have a choice between a risky route and a safer one. To accept this obligation may imply that couples should always opt for adoption or oocyte donation, which, after all, could often be considered the safest routes. Safety indeed is an important consideration when opting for a reproductive strategy, as the costs of using an unsafe technique could be rather high - particularly the immaterial costs. However, exclusively focusing on the safety would not do justice to other relevant considerations, such as the wish for a genetically related child or the material costs of a procedure.

One could also argue that the least costly intervention is the intervention that has the best credentials in delivering a baby: the intervention with the highest 'take home baby rate' (THBR). After all, a fast result may reduce both the material costs and the immaterial costs (as people may have less stress). The ethical justification relies on the optimal use of community resources to maximise well-being (Pennings and Ombelet, 2007). However, directing couples to the intervention with the highest THBR may imply directing couples towards PND. After all, the pregnancy rate after IVF is lower than with natural conception (Poulton and Marchington, 2002). This, on its turn, has the disadvantage that couples may be confronted with the difficult decision about whether or not to terminate their pregnancy. Moreover, to make this decision is known to be psychologically burdensome for a couple, as well as 
physically burdensome for the mother - and in chapter 2 we argued that this may even be intensified in the context of mtDNA disease. In other words: the immaterial costs may be fairly high.

If we take a closer look at the filling in of 'the least costs', it becomes clear that this is a highly personal assessment, depending on personal values and perceptions of what constitutes an acceptable way of having a child given the circumstances. Hitherto, there is both in general and in the context of mtDNA disorders no option that does not involve material and immaterial costs. Having to reproduce by means of one of the reproductive interventions as described in this thesis is, in some way, a tragic situation as all interventions offer some serious moral disadvantages. In this sense, choosing a reproductive option may also be perceived as choosing the lesser evil. If, or as long as, not one strategy clearly towers above the others, it seems reasonable to allow the couple to prioritise and balance the pros and cons of the available interventions according to their own values (and these are considerations in which reasonable people may come to different conclusions). Of course, this is not without any qualifications. After all, the moral minimum as defended in this thesis regards the avoidance of a high risk of serious harm for the child, added with continuous efforts to reduce these risks as much as reasonably possible. Furthermore, this conclusion should be considered a provisional fixed point, requiring adjustment when new relevant data would give cause to do so. 
References 

Abramsky L. Counselling around prenatal testing. In: Prenatal Diagnosis: The Human Side-Abramsky L, Chapple J, eds. (2003) London: Nelson Thornes, pp 70-82.

Aleahmad F, Gourabi H, Zeinali B, Ashtiani SK, Baharvand H. Separation of X- and Y-bearing human spermatozoa by sperm isolation medium gradients evaluated by FISH. Reprod Biomed Online (2009) 18:475-478.

American Society for Reproductive Medicine. Sex selection and preimplantation genetic diagnosis. Fertil Steril (1999) 72:595-598.

American Society for Reproductive Medicine. Preconception gender selection for nonmedical reasons. Fertil Steril (2001) 75:861-864.

Amor DJ and Cameron C. PGD gender selection for non-Mendelian disorders with unequal sex incidence. Hum Reprod (2008) 23:729-734.

Arras JD. AIDS and reproductive decisions: having children in fear and trembling. Milbank Q (1990) 68:353-382.

Asselt MBA van and Vos EIL. The Precautionary Principle and the Uncertainty Paradox. J Risk Research (2006) 9(4):313-336.

Barnett A and McKie R. Babies with three parents ahead. The Observer (2004) October 17.

Barritt JA, Brenner CA, Malter HE, Cohen J. Mitochondria in human offspring derived from ooplasmic transplantation. Hum Reprod (2001a) 16:513-516.

Barritt JA, Brenner CA, Malter HE, Cohen J. Rebuttal: interooplasmic transfers in human. Reprod Biomed Online (2001b) 3:47-48.

Barritt JA, Willadsen S, Brenner C, Cohen J. Cytoplasmic transfer in assisted reproduction. Hum Reprod Update (2001c) 7:428-435.

Baruch S, Huang A, Pritchard D, Kalfoglou A, Javitt G, Borchelt R, Scott J, Hudson K. Human Germline Genetic Modification: Issues and Options for Policymakers (2005) Washington DC: Genetics \& Public Policy Center.

Bayne T and Kolers A. Toward a pluralistic account of parenthood. Bioethics (2003) $17: 221-242$.

Bayne T and Kolers A. Parenthood and Procreation. In: The Stanford Encyclopedia of Philosophy-Zalta EN, ed. (2006) (Winter 2003 Edition).

http://plato.stanford.edu/entries/parenthood/

Beauchamp TL and JF Childress. Principles of Biomedical Ethics (2009) New York and Oxford: Oxford University Press (6 ${ }^{\text {th }}$ edition).

Benatar D. Reproductive freedom and risk. Hum Reprod (2006) 21:2491-2493. 
Bewley S. Ethical issues in prenatal diagnosis. In: Prenatal Diagnosis. The Human Side-Abramsky L, Chapple J, eds. (2003) London, pp 1-16.

Beyleveld D and Brownsword R. Human dignity in bioethics and biolaw (2001) Oxford: Oxford University Press.

Bickerstaff $\mathrm{H}$, Flinter F, Yeong CT, Braude P. Clinical application of preimplantation genetic diagnosis. Hum Fertil (2001) 4:24-30.

Blerkom J van, Sinclair J, Davis P. Mitochondrial transfer between oocytes: potential applications of mitochondrial donation and the issue of heteroplasmy. Hum Reprod (1998) 13:2857-2868.

Blok RB, Gook DA, Thorburn DR, Dahl HHM. Skewed segregation of the mtDNA nt 8993 (T-G) mutation in human oocytes. Am J Hum Genet (1997) 60:1495-1501.

Blyth E, Cameron C. The welfare of the child. An emerging issue in the regulation of assisted conception. Hum Reprod (1998) 13:2339-2341.

Boivin $\mathrm{J}$ and Pennings $\mathrm{G}$. Parenthood should be regarded as a right. Arch Dis Child (2005) 90:784-785.

Bonnicksen AL. Ethical and policy issues in human embryo twinning. Cambr $Q$ Healtc Ethics (1995) 4:268-284.

Bonnicksen AL. The politics of germline therapy. Nat Genet (1998) 19:10-11.

Borry P, Stultiens L, Nys H, Cassiman JJ, Dierickx K. Presymptomatic and predictive genetic testing in minors: a systematic review of guidelines and position papers. Clin Genet (2006) 70:374-381.

Bouchet C, Steffann J, Corcos J, Monnot S, Paquis V, Rötig A, Lebon S, Levy P, Royer G, Giurgea I, Gigarel N, Benachi A, Dumez Y, Munnich A, Bonnefont JP. Prenatal diagnosis of MELAS syndrome: contribution to understanding mitochondrial DNA segregation during human embryo fetal development. J Med Genet (2006) 43:788-792.

Bowles EJ, Campbell KH, St John JC. Nuclear transfer: preservation of a nuclear genome at the expense of its associated mtDNA genome(s). Curr Top Dev Biol (2007) 77:251-290.

Bowles EJ, Tecirlioglu RT, French AJ, Holland MK, St John JC. Mitochondrial DNA transmission and transcription after somatic cell fusion to one or more cytoplasts. Stem Cells (2008) 26:775-782.

Boxall A, Turner L. Problems surrounding late prenatal diagnosis. In: Prenatal Diagnosis. The Human Side-Abramsky L, Chapple J, eds. (2003) London, pp 122137. 
Braude P. Preimplantation genetic diagnosis for genetic susceptibility. N Engl J Med (2006) 6:541-543.

Braude P. One child at a time. Reducing multiple births after IVF (2007) Report of the Expert Group on Multiple Birth after IVF. http://www.hfea.gov.uk/docs/MBSET_report_Final_Dec_06.pdf

Bredenoord AL, Pennings G, Smeets HJ, de Wert G. Dealing with uncertainties: ethics of prenatal diagnosis and preimplantation genetic diagnosis to prevent mitochondrial disorders. Hum Reprod Update (2008a) 14:83-94.

Bredenoord AL, Dondorp WJ, Pennings G, de Die-Smulders CEM, de Wert G. PGD to reduce reproductive risk: the case of mitochondrial DNA disorders. Hum Reprod (2008b) 23:2392-2401.

Bredenoord AL, Pennings G, de Wert G. Ooplasmic transfer and nuclear transfer to prevent mitochondrial DNA disorders: conceptual and normative issues. Hum Reprod Update (2008c) 14:669-678.

Bredenoord AL, Dondorp W, Pennings G, de Die-Smulders C, Smeets B, de Wert G. Preimplantation genetic diagnosis for mitochondrial DNA disorders. Ethical guidance for clinical practice. Eur J Hum Genet (2009) 17:1550-1559

Brenner CA. Ooplasmic transplantation. J Assis Reprod Genet (2004) 21:27-28.

Brenner CA, Barritt JA, Steen Willadsen DVM, Cohen J. Mitochondrial DNA heteroplasmy after human ooplasmic transplantation. Fertil Steril (2000) 74:573-578.

Brewaeys A. How to care for the children? The need for large scale follow-up studies. Hum Reprod (1998) 13:2347-2349.

Briggs DA, Power NJ, Lamb V, Rutherford AJ, Gosden RG. Amplification of DNA sequences in polar bodies from human oocytes for diagnosis of mitochondrial disease. The Lancet (2000) 335:1520-1521.

British Medical Association. Human 'cloning': a discussion paper for the World Medical Association (1999) http://www.bma.org.uk/ap.nsf/Content/Humancloning

Britten N. Qualitative interviews in medical research. BMJ (1995) 311:251-253.

Brock DW. Cloning human beings: an assessment of the ethical issues pro and con. In: Clones and Clones. Facts and Fantasies About Human Cloning-Nussbaum MC, Sunstein CR, eds. (1998) New York and London: W.W. Norton \& Company, pp 141164.

Brock DW. Human Cloning and Our Sense of Self. Science (2002) 296(5566):314316. 
Brown DT, Samuels DC, Michael EM, Turnbull DM, Chinnery PF. Random genetic drift determines the level of mutant mtDNA in human primary oocytes. Am J Hum Genet (2001) 68:533-536.

Brown DT, Herbert M, Lamb VK, Chinnery PF, Taylor RW, Lightowlers RN, Craven L, Cree L, Gardner JL, Turnbull DM. Transmission of mitochondrial DNA disorders: possibilities for the future. The Lancet (2006) 368:87-89.

Buchanan A, Brock DW, Daniels N, Wikler D. From Chance to Choice: Genetics and Justice (2000) Cambridge: Cambridge University Press.

Burg W van der. The slippery-slope argument. J Clin Ethics (1992) 3:256-268.

Burg $\mathrm{W}$ van der and $\mathrm{T}$ van Willigenburg. Introduction. In: Reflective EquilibriumBurg W van der and van Willigenburg T, eds. (1998) Deventer: Kluwer, pp 1-25.

Burgess JA. The great slippery-slope argument. J Med Ethics (1993) 129:169-174.

Burley J and Harris J. Human cloning and child welfare. J Med Ethics (1999) 25:108113.

Caldwell PHY, Murphy SB, Butow PN, Craig JC. Clinical trials in children. The Lancet (2004) 364:803-811.

Campbell KHS, McWhir J, Ritchie WA, Wilmut I. Sheep cloned by nuclear transfer from a cultured cell line. Nature (1996) 380:64-66.

Carelli V, Baracca A, Barogi S, Pallotti F, Valentino ML, Montagna P, Zeviani M, Pini A, Lenaz G, Baruzzi A, Solaini G. Biochemical-clinical correlation in patients with different loads of the mitochondrial DNA T8993G mutation. Arch Neurol (2002) 59:264-270.

Caulfield $\mathrm{T}$ and Brownsword R. Human dignity: a guide to policy making in the biotechnology era? Nature Rev Genet (2006) 7:72-76.

Cavaghan C. Defending the Genetic Supermarket (2007) London: Routledge.

Chen $\mathrm{CH}$, Chen IC, Wang YC, Liu JY, Wu GJ, Tzeng CR. Boy born after gender preselection following successive gestational androgen excess of maternal luteoma and female disorders of sex development. Fertil Steril (2008) 91:2732.e5-7.

Chen SH, Escudero T, Cekleniak NA, Sable DB, Garrisi MG, Munné S. Patterns of ovarian response to gonadotropin stimulation in female carriers of balanced translocation. Fertil Steril (2005) 83:1504-1509.

Chervenak FA, McCullough LB, Campbell S. Is third trimester abortion justified? $\mathrm{Br} J$ Obstet Gyn (1995) 102:434-435. 
Chinnery PF, Howell N, Lightowlers RN, Turnbull DM. MELAS and MERFF. The relationship between maternal mutation load and the frequency of clinically affected offspring. Brain (1998) 121:1889-1894.

Chinnery PF, Taylor DJ, Brown DT, Manners D, Styles P, Lori R. Very low levels of the mtDNA A3243G mutation associated with mitochondrial dysfunction in vivo. Ann Neurol (2000) 47:381-384.

Chinnery PF and Turnbull DM. Epidemiology and treatment of mitochondrial disorders. Am J Med Genet (Semin Med Genet) (2001) 106:94-101.

Chinnery PF. Inheritance of mitochondrial disorders. Mitochondrion (2002) 2:149155.

Chinnery PF, DiMauro S, Shanske S, Schon EA, Zeviani M, Mariotti C, Carrara F, Lombes $A$, Laforet $P$, Ogier $H$, Jaksch $M$, Lochmüller $H$, Horvath $R$, Deschauer $M$, Thorburn DR, Bindoff LA, Poulton J, Taylor RW, Matthews JN, Turnbull DM. Risk of developing a mitochondrial DNA deletion disorder. The Lancet (2004) 363:592-596.

Chinnery PF, Majamaa K, Turnbull D and Thorburn D. Treatment for mitochondrial disorders. Cochrane Database of Systematic Review (2006) Art. No.: CD004426. DOI: 10.1002/14651858.CD004426.pub2.

Chou YJ, Ou CY, Hsu TY, Liou CW, Lee CF, Tso DJ, Wei YH. Prenatal diagnosis of a fetus harboring an intermediate load of the A3243G mtDNA mutation in a maternal carrier diagnosed with MELAS syndrome. Prenat diagn (2004) 24:367-370.

Ciafaloni E, Santorelli FM, Shanske S, Deonna T, Roulet E, Janzer C, Pescia G, DiMauro S. Maternally inherited Leigh syndrome. J Pediatr (1993) 122:419-422.

Clarke A. Genetic screening and counselling. In: A Companion to Bioethics-Kuhse $\mathrm{H}$, Singer P, eds. (1998a) Blackwell Companions to Philosophy, Blackwell Publishers Ltd.

Clarke A. The Genetic Testing of Children (1998b) Oxford and Washington, DC: Bios Scientific Publishers.

Cohen CB. New Ways of Making Babies. The Case of Egg Donation. The National Advisory Board on Ethics in Reproduction. (1996) Indiana University Press Bloomington and Indianapolis.

Cohen $\mathrm{J}$ and Tomkin $\mathrm{G}$. The science, fiction, and reality of embryo-cloning. Kennedy Inst Ethics (1994) 4:193-203.

Cohen J, Scott R, Schimmel T, Levron J, Willadsen S. Birth of infant after transfer of anucleate donor oocyte cytoplasm into recipient eggs. The Lancet (1997) 350:186187. 
Cohen J, Scott R, Alikani M, Schimmel T, Munné S, Levron J, Wu L, Brenner C, Warner C, Willadsen S. Ooplasmic transfer in mature human oocytes. Mol Hum Reprod (1998) 4:269-280.

Cohen J, Wells D, Munné S. Removal of 2 cells from cleavage stage embryos is likely to reduce the efficacy of chromosomal tests that are used to enhance implantation rates. Fertil Steril (2007) 87:496-503.

Cole-Turner R. Human Limits. Theological Perspectives on Germ-Line Modification. In: Designing our Descendants. The Promise and Perils of Genetic Modification Chapman AR, Frankel MS, eds. (2003) Baltimore and London: The Johns Hopkins University Press, pp 188-198.

Combelles $\mathrm{CMH}$. What are the trade-offs between one-cell and two-cell biopsies of preimplantation embryos? Hum Reprod (2008) 23:493-498.

Commissie Doek. Advies medisch-wetenschappelijk onderzoek met kinderen in Nederland. Den Haag, 26 november 2009 [in Dutch].

Council of Europe. Convention for the Protection of Human Rights and Dignity of the Human Being with regard to the Application of Biology and Medicine: Convention on Human Rights and Biomedicine (1997) Orvieto. ETS no 164.

Council of Europe. Additional Protocol to the Convention for the Protection of Human Rights and Dignity of the Human Being with regard to the Application of Biology and Medicine, and the Prohibition of Cloning Human Beings (1998) Paris. ETS no 168.

Council for International Organizations of Medical Sciences. International Ethical Guidelines for Biomedical Research Involving Human Subjects (2002) Geneva: CIOMS.

Craven L, Tuppen HA, Greggains GD, Harbottle SJ, Murphy JL, Cree LM, Murdoch AP, Chinnery PF, Taylor RW, Lightowlers RN, Herbert M, Turnbull DM. Pronuclear transfer in human embryos to prevent transmission of mitochondrial DNA disease. Nature (2010) advance online publication 14 April 2010 DOI:10.1038/nature08958

Cree LM, Samuels DC, de Sousa Lopes SC, Rajasimha HK, Wonnapinij P, Mann JR, Dahl $\mathrm{HH}$, Chinnery PF. A reduction of mitochondrial DNA molecules during embryogenesis explains the rapid segregation of genotypes. Nat Genet (2008) 40:249-54.

Dahl HHM, Thorburn DR, White SL. Towards reliable prenatal diagnosis of mtDNA point mutations: studies of nt8993 mutations in oocytes, fetal tissues, children and adults. Hum Reprod (2000) 15(Suppl 2):246-255.

Dahl HHM and Thorburn DR. Mitochondrial diseases: beyond the magic circle. Am J Med Genet (Semin Med Genet) (2001) 106:1-3. 
Dawkins R. What's Wrong with Cloning? In: Clones and Clones. Facts and Fantasies Human Cloning-Nussbaum MC, Sunstein CR, eds. (1998) New York and London: W.W. Norton \& Company, pp 54-66.

Dean NL, Battersby BJ, Ao A, Gosden RG, LinTan S, Shoubridge EA. Prospect of preimplantation genetic diagnosis for heritable mitochondrial DNA diseases. $\mathrm{Mol}$ Hum Rep (2003) 9:631-638.

DeGrazia D. Human Identity and Bioethics (2005) Cambridge: Cambridge University Press.

Dekker TJ. The Illiberality of Perfectionist Enhancement. Med Health Care Philos (2009) 12: 91-8.

Delden JJM van and van Thiel G (1998) Reflective equilibrium as a normativeempirical model in bioethics. In: Reflective Equilibrium-Burg $\mathrm{W}$ van der and van Willigenburg T, eds. (1998) Deventer: Kluwer, pp 251-259.

Denzin NK and Lincoln YS, eds. The Sage Handbook of Qualitative Research (2005) Thousand Oaks: Sage Publications, $3^{\text {rd }}$ edition.

Devolder K. Creating and sacrificing embryos for stem cells. J Med Ethics (2005) 31:366-370.

Devolder K. What's in a name? Embryos, entities and ANTities in the stem cell debate. J Med Ethics (2006) 32:43-48.

DiMauro S, Hirano M, Schon EA. Approaches to the treatment of mitochondrial diseases. Muscle Nerve (2006) 34:265-283.

Dondorp WJ and de Wert GMWR. Embryonic Stem Cells Without Moral Pain? (2005) The Hague: Centre for Ethics and Health/Health Council of the Netherlands.

Dondorp WJ and de Wert GMWR. On waving the embryos banner. Embryonic stem cell research and moral reasoning in recent reports from the USA, the Netherlands and France. In: Ethics, Law and Society-Gunning J, Holm S, eds. (2007) III. Aldershot: Ashgate.

Draper $\mathrm{H}$ and Chadwich $\mathrm{R}$. Beware! Preimplantation genetic diagnosis may solve some old problems but it also raises new ones. J Med Ethics (1999) 25:114-120.

Dresser R. Preimplantation genetic diagnosis as medical innovation: reflections from The President's Council on Bioethics. Fertil Steril (2006) 85:1633-1637.

Duncan RE and Delatycki MB. Predictive genetic testing in young people for adultonset conditions: where is the empirical evidence? Clin Genet (2006) 69:8-16.

Duncan RE, Gillam L, Savulescu J, Williamson R, Rogers JG, Delatycki MB. "Holding your breath": interviews with young people who have undergone predictive genetic testing for Huntington disease. Am J Med Genet part A (2007) 143A:1984-1989. 
Dworkin R. Life's Dominion. An Argument about Abortion, Euthanasia, and Individual Freedom (1993) New York: Random House.

Editorial Nature. New sources of sex cells. Nature (2008) 452:913.

Eisenberg VH and Schenker JG. Pregnancy in the older woman: scientific and ethical aspects. Int J Gyn Obst (1997) 55:163-169.

Elliott HR, Samuels DC, Eden JA, Relton CL, Chinnery PF. Pathogenic mitochondrial DNA mutations are common in the general population. Am J Hum Genet (2008) 83(2):254-60.

Enns GM, Bai RK, Beck AE, Wong LJ. Molecular-clinical correlations in an family with variable tissue mitochondrial DNA T8993G mutant load. Mol Genet Metab (2006) 88:364-371.

ESHRE PGD Consortium. Best practice guidelines for clinical preimplantation genetic diagnosis (PGD) and preimplantation screening (PGS). Hum Reprod (2005) 20:3548.

European Group on Ethics in Science and New Technologies to the European Commission. Opinion on the ethical aspects of nanomedicine (2007) Opinion no 21. Luxembourg: Office for Official Publications of the European Communities.

Evers-Kieboom G. Predictief testen van minderjarigen voor aandoeningen die later in het leven tot uiting komen: psychologische aspecten en multidisciplinaire aanpak. Tijdschr voor Geneeskunde (2006) 62:463-471 [in Dutch]

Faivre L, Cormier-Daire V, Chrétien D, von Klesit-Retzow JC, Amiel J, Dommergues M, Saudubray JM, Dumez Y, Rötig A, Rustin P, Munnich A. Determination of enzyme activities for prenatal diagnosis of respiratory chain deficiency. Prenat Diagn (2000) 20:732-737.

Fasouliotis SJ and Schenker JG. Preimplantation genetic diagnosis principles and ethics. Hum Reprod (1998) 13:2238-2245.

Fauser BCJM, Devroey $\mathrm{P}$, Macklon NS. Multiple birth resulting from ovarian stimulation for subfertility treatment. The Lancet (2005) 365:1807-1816.

Feinberg J. The Child's Right to an Open Future. In: Whose Child? Children's Rights, Parental Authority, and State Power-Aiken W, LaFollette H, eds. (1980) Totowa, NJ: Rowman and Littlefield. (Reprinted in Freedom\&Fulfillment 1992).

Fost N, Chudwin D, Wikler D. The limited moral significance of 'fetal viability'. Hastings Cent Rep (1980) 10:10-13.

Fukuyama F. Our Posthuman Future. Consequences of the Biotechnology Revolution (2002) New York: Picador.

Fulka J, Fulka H, St John JC. Transmission of mtDNA disorders: possibilities for the elimination of mutated mitochondria. Cloning Stem Cells (2007) 9:47-50. 
Fuscaldo G. What makes a parent? It is not black or white. J Med Ethics (2003) 29:66-67.

Fuscaldo G. Genetic ties: are they morally binding? Bioethics (2006) 20:64-76.

Gans Epner JE, Jonas HJ, Seckinger DL. Late-term Abortion. JAMA (1998) 280:724729.

Gardener SM. A Core Precautionary Principle. Journal of Political Philosophy (2006) 14:33-60.

Gardner JL, Craven L, Turnbull DM, Taylor RW. Experimental strategies towards treating mitochondrial DNA disorders. Biosci Rep (2007) 27:139-150.

Garret C and Margerison L. Difficult decisions in prenatal diagnosis. In: Prenatal Diagnosis. The Human Side-Abramsky L, Chapple J, eds. (2003) London, pp 146163.

Gaylin W and Jennings B. The Perversion of Autonomy. Coercion and Constraints in a Liberal Society (2003) Washington, DC: Georgetown University Press.

Geraedts J and de Wert G. Preimplantation Genetic Diagnosis. Clin Genet (2009) 76(4):315-25.

German National Ethics Council. Genetic Diagnosis Before and During Pregnancy (2003) Berlin: Nationaler Ethikrat. Opinion.

German National Ethics Council. Polar Body Diagnosis (2005) Berlin: Nationaler Ethikrat. Opinion.

Gillon R. Human reproductive cloning - a look at the arguments against it and a rejection of most of them. $J R$ Soc Med (1999) 2:3-12.

Gillon R. Is there a 'new ethics of abortion'? J Med Ethics (2001) 27:ii5-ii9.

Glover J. Choosing children. Genes, Disability, and Design (2006) Oxford: Clarendon Press.

Golombok S. New families, old values: considerations regarding the welfare of the child. Hum Reprod (1998) 13:2342-2347.

Golombok S, Murray C, Jadva V, Lycett E, MacCullum F, Rust J. Non-genetic and non-gestational parenthood: consequences for parent-child relationships and the psychological well-being of mothers, fathers and children at age 3. Hum Reprod (2006) 21:1918-24.

Goossens V, De Rycke M, De Vos A Staessen C, Michiels A, Verpoest W, Van Steirteghem A, Bertrand C, Liebaers I, Devroey P, Sermon K. Diagnostic efficiency, embryonic development and clinical outcome after the biopsy of one or two blastomeres for preimplantation genetic diagnosis. Hum Reprod (2008) 23:481-492. 
Graaf van der R and van Delden JJM. Clarifying appeals to dignity in medical ethics from an historical perspective. Bioethics (2009) 23:151-160.

Graff C, Wredenberg A, Silva JP, Bui TH, Borg K, Larsson NG. Complex genetic counselling and prenatal analysis in a woman with external ophthalmoplegia and deleted mtDNA. Prenat Diagn (2000) 20:426-431.

Graumann S and Haker H. Some conceptual and ethical comments on egg cell nuclear transfer. Polit Life Sci (1998) 17-19.

Grimes DA. The continuing need for late abortions. JAMA (1998) 280:747-750.

Handelingen Tweede Kamer. 2005-2006 30300 XVI, nr. 136 [in Dutch]

Guest G, Brunce A, Johnson L. How many interviews are enough? An experiment with data saturation and variability. Field Methods (2006) 18:59-82.

Haas RH, Parikh S, Falk MJ, Saneto RP, Wolf NI, Darin N, Cohen BH. Mitochondrial disease: a practical approach for primary care physicians. Pediatrics (2007) 120:1326-1333.

Habermas J. The Future of Human Nature (2003) Cambridge: Polity Press.

Hancock MD, Conradt DP, Peters BG, Safran W, White S, Zariski R. Politics in Europe (2002) Hampshire: Palgrave Macmillan ( $3^{\text {rd }}$ edition).

Handyside $\mathrm{AH}$, Kontogianni EH, Hardy K, Winston RM. Pregnancies from biopsied human preimplantation embryos sexed by Y-specific DNA amplification. Nature (1990) 344(6268):768-70.

Harding AE, Holt IJ, Sweeney MG, Brockington M, Davis MB. Prenatal diagnosis of mitochondrial DNA8993 T-G disease. Am J Hum Genet (1992) 50:629-633.

Harris J. "Goodbye Dolly?" The ethics of human cloning. J Med Ethics (1997) 23(6):353-60.

Harris J. Rights and reproductive choice. In: The Future of Human Reproduction. Ethics, Choice and Regulation-Harris J, Holm S, eds. (1998) Oxford: Clarendon Press, pp 5-37.

Harris J. Doctor's orders, rationality and the good life: commentary on Savulescu. $J$ Med Ethics (1999) 25:127-129.

Harris J. The welfare of the child. Health Care Anal (2000) 8:27-34.

Harris J. Enhancing Evolution. The Ethical Case for Making Better People (2007) Princeton and Oxford: Princeton University Press.

Harris M, Winship I, Spriggs M. Controversies and ethical issues in cancer-genetics clinics. Lancet Neurology (2005) 6:301-310. 
Harper J, ed. Preimplantation Genetic Diagnosis (2009) Cambridge: Cambridge University Press, $2^{\text {nd }}$ Edition.

Harper JC, De Die-Smulders C, Goossens V, Harton G, Moutou C, Repping S, Scriven PN, SenGupta S, Traeger-Synodinos J, Van Rij MC, Viville S, Wilton L, Sermon KD. ESHRE PGD consortium data collection VII: cycles from January to December 2004 with pregnancy follow-up to October 2005. Hum Reprod (2008) 23:741-755.

Hawes SM, Sapienza C, Latham KE. Ooplasmic donation in humans. The potential for epigenetic modifications. Hum Reprod (2002) 17(4):850-852.

Health Council of the Netherlands. IVF-related research (1998) Rijswijk: Health Council of the Netherlands. Publication no 1998/08E.

Health Council of the Netherlands. Nuclear Transplantation in Cases of Mutations in Mitochondrial DNA (2001) The Hague: Health Council of the Netherlands. Publication no $2001 / 07$.

Health Council of the Netherlands. Ethics and Health Monitoring Report (2003) The Hague: Health Council of the Netherlands. Publication no 2003/08.

Health Council of the Netherlands. Sex selection for non-medical reasons (1995) The Hague: Health Council of the Netherlands. Publication no 1995/11E.

Health Council of the Netherlands. Pre-implantation genetic diagnosis (2006) The Hague: Health Council of the Netherlands. Publication no 2006/01.

Heijnen E, Eijkemans M, De Klerk C, Polinder S, Beckers NG, Klinkert ER, Broekmans FJ, Passchier J, Te Velde ER, Macklon NS, Fauser BC. A mild treatment strategy for in-vitro fertilisation: a randomised non-inferiority trial. The Lancet (2007) 369:743-749.

Hirschler B. Scientists create three-parent embryos. Thomson Reuters (2008) 5 February.

http://www.reuters.com/article/scienceNews/idUSL0516465820080205

Holm S. Ethical issues in pre-implantation diagnosis. In: The Future of Human Reproduction. Ethics, Choice and Regulation-Harris J, Holm S, eds. (1998) Oxford: Clarendon Press. 176-190.

Holm S. A life in shadows: one reason why we should not clone humans. Camb $Q$ Healthc Ethics (1998) 7:160-162.

Holm S and Harris J. Precautionary principle stifles discovery. Nature (1999) 400:398.

Hope T and McMillan J. Ethical problems before conception. The Lancet (2003) 21;361(9375):2164. 
Howards CV and Saunders PT. Sensible precautions make good science. Nature (1999) $401: 207$.

Huang CC, Cheng TZ, Chang HH, Chang CC, Chen Cl, Liu J, Lee MS. Birth after the injection of sperm and the cytoplasm of tripronucleate zygotes into metaphase II oocytes in patients with repeated implantation failure after assisted fertilization procedures. Fertil Steril (1999) 72:702-706.

Hudson G, Carelli V, Spruijt L, Gerards M, Mowbray C, Achilli A, Pyle A, Elson J, Howell N, La Morgia C, Valentino MC, Huoponen K, Savontaus ML, Nikoskelainen E, Sadun AA, Salomao SR, Belfort R, Griffiths P, Man PYW, de Coo RFM, Horvath R, Zeviani M, Smeets HJT, Torroni A, Chinnery PF. Clinical expression of leber hereditary optic neuropathy is affected by the mitochondrial DNA-haplogroup background. Am J Hum Genet (2007) 81:228-233.

Hughes J. How Not to Criticize the Precautionary Principle. Journal of Medicine and Philosophy (2006) 31:447-464.

Human Fertilisation and Embryology Authority. Guidance on Preimplantation Testing (2003) http://www.hfea.gov.uk/en/601.html.

Human Fertilisation and Embryology Authority R0153. http://www.hfea.gov.uk/docs/R0153_How the decision_was made to licence this research_project_2_pdf.

Human Fertilisation and Embryology Authority. Code of Practice (2003) 6th edn.

Huoponen K, Puomilla A, Savontaus ML, Mustonen E, Kronqvist E, Nikoskelainen E. Genetic counseling in Leber hereditary optic neuropathy (LHON). Acta Ophthalmol Scand (2002) 80:38-43.

Hyun I. Fair payment or undue inducement? Nature (2006) 442:629-630.

Jacobs LJAM, de Coo IFM, Nijland JG, Galjaard RJH, Los FJ, Schoonderwoerd K, Niermeijer MF, Geraedts JPM, Scholte HR, Smeets HJM. Transmission and prenatal diagnosis of the T9176C mitochondrial DNA mutation. Mol Hum Rep (2005) 11:223228.

Jacobs LJAM, de Wert G, Geraedts JPM, de Coo IFM, Smeets HJM. The transmission of OXPHOS disease and methods to prevent this. Hum Reprod Update (2005) 12:119-136.

Jacopini G, Decruyenaere M, Harper R, Simpson SA. Case histories of prenatal testing for Huntington's disease. In: Prenatal Testing for Late-Onset Neurogenetic Disease-Evers-Kiebooms G, Zoeteweij MW, Harper PS, eds. (2002) Oxford: BIOS Scientific Publishers Ltd, pp 11-24.

Jonas H. Philosophical Essays: from Ancient Creed to Technological Man (1974) New York: Prentice Hall. 
Jonsen AR and Toulmin S. The Abuse Of Casuistry. A History of Moral Reasoning (1998) Berkeley and Los Angelos: University of California Press.

Kagawa $\mathrm{Y}$ and Hayashi Jl. Gene therapy of mitochondrial diseases using human cytoplasts. Gene Ther (1997) 4:6-10.

Karabinus DS. Flow cytometric sorting of human sperm: MicroSort clinical trial update. Theriogenology (2009) 71:74-79.

Kass LR. Life, Liberty, and the Defense of Dignity. The Challenge for Bioethics (2002) San Francisco: Encounter Books.

Kass LR. Defending Human Dignity. In: Human Dignity and Bioethics. President's Council on Bioethics (2008) Washington DC: The President's Council on Bioethics, pp 297-331.

Katz Rothman B. The Tentative Pregnancy. Prenatal Diagnosis and the Future of Motherhood (1986) Viking Penguin Inc.

Kimmelman J. The ethics of human gene transfer. Nature (2008) 9:239-244.

King DS. Preimplantation genetic diagnosis and the 'new' eugenics. J Med Ethics (1999) 25:176-182.

Klipstein S. Preimplantation genetic diagnosis: technological promise and ethical perils. Fertil Steril (2005) 83:1347-1353.

Kolers A and Bayne T. "Are you my mommy?" On the genetic basis of parenthood. $J$ Applied Philos (2001) 18:273-85.

Kopelman LM, Resnick D, Weed DL. What is the Role of the Precautionary Principle in the Philosophy of Medicine and Bioethics? J Med Philos (2004) 29:255-258.

Korenromp MJ, ledema-Kuiper HR, van Spijker HG, Christiaens GCML, Bergsma J. Termination of pregnancy on genetic grounds: coping with grieving. $J$ Psychosom Obstet Gynaecol (1992) 13:93-105.

Krey L, Liu H, Zhang J, Grifo J. Strategies to Improve Pregnancy Outcome. Ann N Y Acad Sci (2001) 943:26-33.

Kuehn BM. Scientists Probe Method to Prevent Inherited Mitochondrial Gene Diseases. JAMA (2009) 302:1409.

Kuhse $\mathrm{H}$. Patient-centred ethical issues raised by the procurement and use of gametes and embryos in assisted conception. In: Current Practices and Controversies in Assisted Reproduction-Vayena E, Rowe PJ, Griffin PD, eds. (2002) Geneva: World Health Organization, pp 305-319.

Kuliev A and Verlinsky Y. Preimplantation genetic diagnosis in assisted reproduction. Expert Rev Mol Diagn (2005a) 5:499-505. 
Kuliev A and Verlinsky Y. Place of preimplantation diagnosis in genetic practice. $\mathrm{Am}$ $J$ Med Genet (2005b) 134A:105-110.

Lamb D. Down the Slippery Slope (1988) London: Croom Helm.

Lanzendorf SE, Mayer JF, Toner J, Oehninger S, Saffan DS, Muasher S. Pregnancy following transfer of ooplasm from cryopreserved-thawed donor oocytes into recipient oocytes. Fertil Steril (1999) 71:575-577.

Lavery SA, Aurell R, Turner C, Castello C, Veiga A, Barri PN, Winston RM. Preimplantation genetic diagnosis: patient's experiences and attitudes. Hum Reprod (2002) 17:2464-2467.

Laurie G. In defense of ignorance: genetic information and the right not to know. Eur J Health Law (1999) 6:119-132.

Lashwood A. Preimplantation genetic diagnosis. In: Prenatal Diagnosis. The Human Side-Abramsky L, Chapple J, eds. (2003) London: Nelson Thornes, pp 107-121.

Lease LR, Winnier DA, Williams JT, Dyer TD, Almasy L, Mahaney MC. Mitochondrial genetic effects on latent class variables associated with susceptibility to alcoholism. BMC Genet (2005) 6(Suppl I):S158.

Leshinsky-Silver E, Perach M, Basilevsky E, Hershkovitz E, Yanoov-Sharav M, Lerman-Sagie T, Lev D. Prenatal exclusion of Leigh syndrome due to T8993C mutation in the mitochondrial DNA. Prenat Diagn (2003) 23:31-33.

Liang CG, Han Z, Cheng Y, Zhong Z, Latham KE. Effects of ooplasm transfer on paternal genome function in mice. Hum Reprod (2009) 24(11):2718-28.

Lloyd AJ. The extent of patient's understanding of the risk of treatments. Qual Health Care (2001) 10(Suppl I): i14-i18.

Macklin R. Artificial Means of Reproduction and Our Understanding of the Family. Hastings Cent Rep (1991) 21:5-11.

MacMahan J. The Ethics of Killing. Problems at the Margins of Life (2002) Oxford: Oxford University Press.

MacMahan J. Causing disabled people to exist and causing people to be disabled. Ethics (2005) 116:77-99.

Magli MC, Gianaroli L, Grieco N, Cefalu E, Ruvolo G, Ferraretti AP. Cryopreservation of biopsied embryos at the blastocyst stage. Hum Reprod (2006) 21:2656-2660.

Malek J. Understanding Risks and Benefits in Research on Reproductive and Genetic Technologies. J Med Philos (2007) 32:339-358.

Malter HE. Improving eggs: more questions than answers. J Ass Reprod Genet (2002) 19:118-120. 
Malterud K. Qualitative research: standards, challenges, and guidelines. The Lancet (2001) 358:483-488.

Man PYW, Turnbull DM, Chinnery PF. Leber hereditary optic neuropathy. J Med Genet (2002) 39:162-169.

Marchington D, Malik S, Banerjee A, Turner K, Samuels D, Macaulay V, Oakeshott $P$, Fratter C, Kennedy S, Poulton J. Information for genetic management of mtDNA disease: Sampling pathogenic mtDNA mutants in the human germline and in placenta. J Med Genet (2009) Nov 12 [Epub ahead of print].

Marteau TM and Dormandy E. Facilitating informed choice in prenatal testing: how well are we doing? Am J Med Genet (2001) 106(3):185-190.

McFarland R, Taylor RW, Turnbull DM. Mitochondrial Disease - Its Impact, Etiology, and Pathology. Curr Top Dev Biol (2007) 77:113-155.

McGleenan T. Human gene therapy and slippery slope arguments. J Med Ethics (1995) 21:350-355.

McLaren A. IVF: regulation or prohibition? Nature (1989) 342:469-470.

Medical Research Council. Assisted reproduction: a safe, sound future (2004) London: Medical Research Council.

Mertes $\mathrm{H}$ and Pennings $\mathrm{G}$. Oocyte donation for stem cell research. Hum Reprod (2007) 22(3):629-34.

Mertes $\mathrm{H}$ and Pennings $\mathrm{G}$. Embryonic stem cell-derived gametes and genetic parenthood: a problematic relationship. Cambr Q Healthc Ethics (2008) 17(1):7-14.

Midgley M. Biotechnology and monstruosity: why we should pay attention to the 'yuk factor'. Hastings Center Report (2000) 30:7-15.

Mol A. Meer technologie: meer praten. Medisch Contact (1995) 51/52:1652-1654 [in Dutch].

Moreno-Loshuertos R, Acin-Perez R, Fernandez-Silva P, Movilla N, Perez-Martos A, Rodriguez de Cordoba S, Gallardo ME, Enriquez JA. Differences in reactive oxygen species production explain the phenotypes associated with common mouse mitochondrial DNA variants. Nat Genet (2006) 38:1261-8.

Mumford SE, Corrigan E, Hull MGR. Access to assisted reproduction: a framework of regulation. Hum Reprod (1998) 13:2349-2355.

Munnich A, Rotig A, Chretien D, Saudubray JM, Cormier V, Rustin P. Clinical presentation of mitochondrial disorders in childhood. J Inherit Metab Dis (1996) 19:521-527. 
Murray TH. New reproductive technologies and the family. In: New Ways of Making Babies. The Case of Egg Donation-Cohen CB, ed. (1996) Bloomington and Indianapolis: The National Advisory Board on Ethics in Reproduction. Indiana University Press, pp 51-69.

Nagao Y, Totsuka Y, Atomi Y, Kaneda H, Lindahl KF, Imai H, Yonekawa $\mathrm{H}$. Decreased physical performance of congenic mice with mismatch between the nuclear and the mitochondrial genome. Genes Genet Syst (1997) 73:21-27.

Nakada K, Sato A, Hayashi J. Reverse genetic studies of mitochondrial DNA-based diseases using a mouse model. Proc Jpn Acad Ser B Phys Biol Sci (2008) (84)5:155165.

National Bioethics Advisory Commission. The Science and Application of Cloning. In: Clones and Clones. Facts and Fantasies about Human Cloning-Nussbaum MC, Sunstein CR, eds. (1998) New York/London: WW Norton \& Company, pp 29-40.

Niermeijer MF, de Wert G, Dondorp W. Preimplantation genetic diagnosis for cancer. Lancet Oncol (2006) 10:794-795.

Nussbaum MC. Hiding from humanity. Disgust, shame, and the law (2004) Princeton: Princeton University Press.

Nys $\mathrm{H}$, Casabona CMR, Desmet $\mathrm{C}$. Legal aspects of prenatal testing for late-onset neurological diseases. In: Prenatal Testing for Late-Onset Neurogenetic DiseaseEvers-Kiebooms G, Zoeteweij MW, Harper PS, eds. (2002) Oxford: BIOS Scientific Publishers Ltd, pp 83-106.

Olsthoorn-Heim ETM, de Wert GMWR, Winter HB, te Braake ThAM, Heineman MJ, Middelkamp A, Nierse CJ. Evaluatie Embryowet (2006) Den Haag: ZonMw. Reeks evaluatie regelgeving: deel 20 .

Ossorio PN. Inheritable Genetic Modifications: Do We Owe Them to Our Children? In: Designing our Descendants. The Promise and Perils of Genetic ModificationChapman AR, Frankel MS, eds. (2003) Baltimore and London: The Johns Hopkins University Press, pp 252-271.

Parens E and Juengst E. Inadvertently crossing the germ line. Science (2001) 292:397.

Parfit D. Reasons and Persons (1984) Oxford: Oxford University Press.

Parks JA. A closer look at reproductive technology and postmenopausal motherhood. Can Med Assoc J (1996) 154:1189-1191.

Parriego M, Solé M, Aurell R, Barri PN, Veiga A. Birth after transfer of frozen-thawed vitrified biopsied blastocysts. J Assist Reprod Genet (2007) 24:147-149.

Parker M. The welfare of the child. Hum Fertil (2005) 8:13-17. 
Parker M. The best possible child. J Med Ethics (2007) 33:279-283.

Patton M. Qualitative research and evaluation methods (2002) Thousand Oaks, CA: Sage Publications.

Pennings G. Measuring the welfare of the child: in search of the appropriate evaluation principle. Hum Reprod (1994) 14:1146-1150.

Pennings G. Personal desires of patients and social obligations of geneticists: applying preimplantation genetic diagnosis for non-medical sex selection. Prenat diagn (2002) 22:1123-1129.

Pennings G. The physician as an accessory in the parental project of HIV positive people. J Med Ethics (2003) 29:321-324.

Pennings $G$ and de Wert G. Evolving ethics in medically assisted reproduction. Hum Reprod Update (2003) 9:397-404.

Pennings G, Bonduelle M, Liebaers I. Decisional authority and moral responsibility of patients and clinicians in the context of preimplantation genetic diagnosis. Reprod Biomed Online (2003) 5:509-513.

Pennings and Ombelet. Coming soon to your clinic: patient friendly ART. Hum Reprod (2007) 22:2075-9.

Pennings G, de Wert GM, Shenfield F, Cohen J, Tarlatzis B, Devroey P. ESHRE task force on ethics and law 12: oocyte donation for non-reproductive purposes. Hum Reprod (2007) 22:1210-1213.

Pennings G, de Wert G, Shenfield G, Cohen J, Tarlatzis B, Devroey P. ESHRE Task Force on Ethics and Law 13: the welfare of the child in medically assisted reproduction. Hum Reprod (2007a) 22:2585-2588.

Pennings G, de Wert G, Shenfield F, Cohen J, Tarlatzis B, Devroey P. ESHRE Task Force on Ethics and Law 14: equity of access to assisted reproductive technology. Hum Reprod (2008) 23:772-774.

Peters PG. How Safe Is Safe Enough? Obligations to the Children of Reproductive Technology (2004) Oxford: Oxford University Press.

Pettman R, Hurley $\mathrm{T}$, Robinson B, Scott $\mathrm{H}$, Kronick JB. Prenatal diagnosis by amniocentesis and chorionic villus biopsy of mtDNA mutation 8993T>G. J Inherit Metab Dis (2007) 30:404.

Polit DF and Beck CT. Nursing Research: Principles and Methods (2003) Philadelphia: Lippincott Williams \& Wilkins, $7^{\text {th }}$ Edition.

Poulton $\mathrm{J}$ and Turnbull DMA. $74^{\text {th }}$ ENMC international workshop: mitochondrial diseases 19-20 November 1999, Naarden, The Netherlands. Neuromuscul Disord (2000) 10:460-462. 
Poulton $\mathrm{J}$ and Marchington DR. Progress in genetic counselling and prenatal diagnosis of maternally inherited mtDNA disease. Neuromuscul Disord (2000) 10:484-487.

Poulton $J$ and Marchington DR. Segregation of mitochondrial DNA (mtDNA) in human oocytes and in animal models of mtDNA disease: clinical implications. Reproduction (2002) 123(6):751-5.

Poulton J, Kennedy S, Oakeshott P, St John J. Nuclear transfer to prevent mitochondrial DNA diseases. The Lancet (2006) 368:841.

Poulton J, Kennedy S, Oakeshott P, Wells D. Preventing transmission of maternally inherited mitochondrial DNA diseases. BMJ (2009) 338:345-349.

Purdy LM. Assisted reproduction. In: A Companion to Bioethics. Blackwell Companions to Philosophy-Kuhse H, Singer P, eds. (1998) Blackwell Publishers Ltd, pp 163-172.

Prentice ED and Gordon BG. Institutional Review Board Assessment of Risks and Benefit associated with Research. In: Ethical and Policy Issues in Research Involving Human Participants. Volume II: Commissioned Papers and Staff Analysis-National Advisory Bioethics Committee (2001) Bethesda, Maryland, pp 1-16.

President's Council on Bioethics. Human Cloning and Human Dignity. An Ethical Inquiry (2002) Washington, DC: The President's Council on Bioethics.

President's Council on Bioethics. Reproduction and Responsibility. The Regulation of New Biotechnologies (2004) Washington, DC: The President's Council on Bioethics.

President's Council on Bioethics. Human Dignity and Bioethics (2008) Washington DC: The President's Council on Bioethics.

Randerson J. Scientists seek to create 'three-parent babies'. New Scientist (2004) 19 October.

Rawls J. A Theory of Justice (1971) Cambridge: The Belknap Press of Harvard University Press, Revised Edition.

Resnik DB and Langer PJ. Human Germline Gene Therapy Reconsidered. Hum Gene Ther (2001) 12:1449-1458.

Resnik DB. The Precautionary Principle and Medical Decision Making. J Med Philos (2004) 29:281-299.

Rhoden NK. Trimesters and technology: revamping Roe v. Wade. Yale Law J (1986) 96:639-697.

Rhodes R. Why Test Children for Adult-Onset Genetic Diseases? Mt Sinai J Med (2006) 73:609-616. 
Rice M. Very early detection? British group gauges public interest in embryo testing for cancer. J Nat Cancer Inst (2006) 98:156-157.

Roberts RM. Prevention of human mitochondrial (mtDNA) disease by nucleus transplantation into an enucleated donor oocyte. Am J Med Genet (1999) 87:265-66.

Roberts M. The Nonidentity Problem. In: Stanford Encyclopedia of Philosophy-Zalta EN, ed. (2009 Edition) http://plato.stanford.edu/entries/nonidentity-problem/

Roberts MA and Wasserman DT, eds. Harming Future Persons. Ethics, Genetics, and the Nonidentity Problem (2009) Dordrecht Heidelberg London New York: Springer.

Robertson JA. Children of Choice. Freedom and The New Reproductive Technologies (1994) Princeton: Princeton University Press.

Robertson JA. Reconstituting eggs: the ethics of cytoplasm donation. Fertil Steril (1999) 71:219-221.

Robertson JA. Extending preimplantation genetic diagnosis: the ethical debate. Ethical issues in new uses of preimplantation genetic diagnosis. Hum Reprod (2003) 18:465-471.

Robertson S and Savulescu J. Is there a case in favour of predictive genetic testing in young children? Bioethics (2001) 15:26-49.

Roubertoux PL, Sluyter F, Carlier M, Marcet B, Maarouf-Veray F, Chérif C, Marican C, Arrechi P, Godin F, Jamon M, Verrier B, Cohen-Salmon C. Mitochondrial DNA modifies cognition in interaction with the nuclear genome and age in mice. Nature Genet (2003) 35:65-69.

Rubenstein DS, Thomasma DC, Schon EA, Zinaman JC. Germ-line therapy to cure mitochondrial disease: protocol and ethics of in vitro ovum nuclear transplantation. Camb Q Healthc Ethics (1995) 4:316-339.

Sacred Congregation for the Doctrine of Faith. Donum Vitae. Instruction on Respect for Human Life in Its Origin and the Dignity of Procreation. (1987).

Salvi M. Shaping individuality: human inheritable germ line gene modification. Theor Med (2001) 22:527-542.

Sandel M. The Case against Perfection (2007) Cambridge and London: The Belknap Press of Harvard University Press.

Sato A, Kono T, Nakada K, Ishikawa K, Inoue S, Yonekawa H, Hayashi J. Gene therapy for progeny of mito-mice carrying pathogenic mtDNA by nuclear transplantation. Proc Natl Acad Sci U S A (2005) 102:16765-70.

Savulescu J. Should doctors intentionally do less than the best? J Med Ethics (1999) 25:121-126. 
Savulescu J. Procreative beneficence: why we should select the best children. Bioethics (2001) 15:413-426.

Schaefer AM, McFarland R, Blakely EL, He L, Whittaker RG, Taylor RW, Chinnery PF, Turnbull DM. Prevalence of mitochondrial DNA disease in adults. Ann Neurol (2008) 63(1):35-9.

Schapira AHV. Mitochondrial disease. The Lancet (2006) 368:70-82.

Schulman JD and Karabinus DS. Scientific aspects of preconception gender selection. Reprod Biomed Online (2005) 10 Suppl 1:111-5.

Schwartz B. The Paradox of Choice: Why More is Less (2004) New York: Harper Perennial.

Sermon K, Van Steirteghem A, Liebaers I. Preimplantation genetic diagnosis. The Lancet (2004) 363:1633-1641.

Shahine LK, Caughey AB. Preimplantation genetic diagnosis: the earliest form of prenatal diagnosis. Gynecol Obstet Invest (2005) 60:39-46.

Shiloh S, Gerad L, Goldman B. The facilitating role of information provided in genetic counseling for counselees' decisions. Genet Med (2006a) 8(2):116-124.

Shiloh S, Gerad L, Goldman B. Patients' Information Needs and Decision-Making Processes: What Can Be Learned From Genetic Counselees? Health Psychol (2006b) 25:211-219.

Shoubridge EA. Asexual healing. Nature (2009) 461:354-355.

Smith T. Ethics in Medical Research. A Handbook of Good Practice (1999) Cambridge: Cambridge University Press.

Soini S, Ibarreta D, Anastasiadou V, Aymé S, Braga S, Cornel M, Coviello DA, Evers-Kiebooms G, Geraedts J, Gianaroli L, Harper J, Kosztolanyi G, Lundin K, Rodrigues-Cerezo E, Sermon K, Sequeiros J, Tranebjaerg L, Kääriäinen H on behalf of ESHG and ESHRE. The interface between assisted reproductive technologies and genetics: technical, social, ethical and legal issues. Eur J Hum Genet (2006) 14:588645.

Sparrow R. Cloning, parenthood, and genetic relatedness. Bioethics (2006) 20:308318.

Spielthenner G. A Logical Analysis of Slippery Slope Arguments. Health Care Anal advance online publication 9 June 2009; DOI 10.1007/s10728-009-0117-0.

Spikings EC, Alderson J, John JC St. Transmission of mitochondrial DNA following assisted reproduction and nuclear transfer. Hum Reprod Update (2006) 12:401-415. 
Sprang ML, Mark G, Neerhof MG. Rationale for banning abortions late in pregnancy. JAMA (1998) 280:744-747.

Spruijt L. Leber's Hereditary Optic Neuropathy. In: Encyclopedia of Life Sciences (2007) Chichester: John Wiley \& Sons, Ltd. DOI: 10.1002/9780470015902.a0005541.

Steffann J, Frydman N, Gigarel N Burlet P, Ray PF, Fanchin R, Feyereisen E, Kerbrat V, Tachdjian G, Bonnefont JP, Frydman R, Munnich A. Analysis of mtDNA variant segregation during early human embryonic development: a tool for successful NARP preimplantation diagnosis. J Med Genet (2006) 43:244-247.

Steffann J, Gigarel N, Corcos J, Bonniere M, Encha-Razavi F, Sinico M, Prevot S, Dumez Y, Yamgnane A, Frydman R, Munnich A, Bonnefont JP. Stability of the m.8993T>G mutation load during human embryo fetal development has implications for the feasibility of prenatal diagnosis in NARP syndrome. J Med Genet (2007) 44:664-669.

Steinbock B. Preimplantation genetic diagnosis and embryo selection. In: $A$ Companion to Genethics-Burley J, Harris J, eds. (2002) Blackwell Companions to Philosophy, pp 175-190.

Steinbock B. Prenatal testing for adult-onset conditions: cui bono? Reprod Biomed Online (2007) 15(Suppl 2):38-42.

Steinbock B and McClamrock R. When Is Birth Unfair to the Child? Hastings Center Report (1994) 6:15-21.

St John $J$ and Lovell-Badge R. Human-animal cytoplasmic hybrid embryos, mitochondria, and an energetic debate. Nature Cell Biol (2007) 9:988-992.

St John J, Lloyd RE, Bowles EJ, Thomas EC, Shourbagy SE. The consequences of nuclear transfer for mammalian foetal development and offspring survival. A mitochondrial DNA perspective. Reproduction (2004) 127:631-641.

Strauss AL and Corbin J. Basics of qualitative research: techniques and procedures for developing grounded theory (1998). London: Sage Publications.

Strong C. Ethics in Reproductive and Perinatal Medicine. A New Framework (1997) New Haven \& London: Yale University Press.

Sue CM, Quigley A, Katsabanis S Kapsa R, Crimmins DS, Byrne E, Morris JG. Detection of MELAS A3243G point mutation in muscle, blood and hair follicles. $J$ Neurol Sci (1998) 161:36-39.

Sunstein CR. The Paralyzing Principle. Regulation winter (2002-3):32-37.

Sutton A. Prenatal Diagnosis: Confronting the Ethical Issues (1990) London: The Linacre Centre for the Study of the Ethics of Health Care. 
Szebik I. Altering the mitochondrial genome: is it just a technical issue? Response to 'germ line therapy to cure mitochondrial disease: Protocol and ethics of in vitro ovum nuclear transplantation'. Cambr Q Healthc Ethics (1999) 8:369-374.

Tachibana M, Sparman M, Sritanaudomchai H, Ma H, Clepper L, Woodward J, Li Y, Ramsey C, Kolotushkina O, Mitalipov S. Mitochondrial gene replacement in primate offspring and embryonic stem cells. Nature (2009) 461:367-372.

Tatuch Y, Christodoulou J, Feigenbaum A, Clarke JT, Wherret J, Smith C, Rudd N, Petrova-Benedict R, Robinson BH. Heteroplasmic mtDNA mutation (T-G) at 8993 can cause Leigh disease when the percentage of abnormal mtDNA is high. $A m J$ Hum Genet (1992) 50:852-858.

Taylor RW and Turnbull DM. Mitochondrial DNA mutations in human disease. Nat Rev Genet (2005) 6:389-402.

Templeton A. Ooplasmic transfer - proceed with care. N Engl J Med (2002) 346:773775.

Thorburn DR. Mitochondrial disorders: prevalence, myths and advances. J Inherit Metab Dis (2004) 27:349-363.

Thorburn DR and Dahl HHM. Mitochondrial disorders: genetics, counseling, prenatal diagnosis and reproductive options. Am J Med Genet (Semin Med Genet) (2001) 106:101-114.

Thorburn DR, Dahl HHM, Singh KK. The pros and cons of mitochondrial manipulation in the human germ line. Mitochondrion (2001) 1:123-127.

Tooley M. Personhood. In: A Companion to Bioethics-Kuhse H, ed. (1998) Blackwell Publishers Ltd, Blackwell Companions to Philosophy, pp 117-126.

Tooley M. Abortion and Infanticide. In: Bioethics-Kuhse H, Singer P, eds. (1999) Blackwell Publishers Ltd, Blackwell Philosophy Anthologies.

Tuppen HA, Blakely EL, Turnbull DM, Taylor RW (2009) Mitochondrial DNA mutations and human disease. Biochim Biophys Acta (2009) Sept 15 [Epub ahead of print].

United Nations Universal Declaration on the Human Genome and Human Rights (1997) http://portal.unesco.org/en/ev.php-

URL ID=13177\&URL_DO=DO_TOPIC\&URL_SECTION=201.html

United Nations Declaration on Human Cloning (1998) http://daccessdds.un.org/doc/UNDOC/GEN/N04/493/06/PDF/N0449306.pdf?OpenEI ement.

United Nations Educational, Scientific and Cultural Organization. Report of the IBC on Pre-implantation Genetic Diagnosis and Germ-line Intervention (2003) Paris: UNESCO, Division of the Ethics of Science and Technology. 
Verlinsky Y and Kuliev A. The Encyclopedia of Visual Medicine Series (2000) The Parthenon Publishing Group. An Atlas of Preimplantation Genetic Diagnosis.

Visser MRM, Janssen AJGM, Enschedé M, Willems AFMN, Braake ThAM te, Harmsen K, Smets EMA, Haes de JCJM, Gevers JKM. Evaluatie Wet afbreking zwangerschap (2005) Den Haag: ZonMW. Reeks evaluatie wetgeving [in Dutch].

Volmink J, Siegfried NL, van der Merwe L, Brocklehurst P. Antiretrovirals for reducing the risk of mother-to-child transmission of HIV infection. Cochrane Database Syst $\operatorname{Rev}(2007)$ 24. CD003510.

Warren MA. Gendercide. The implications of sex selection (1985) New Yersey: Rowman and Allenheld.

Warren MA. Abortion. In: A Companion to Bioethics-Kuhse H, Singer P, eds. (1998) Blackwell Companions to Philosophy, Blackwell Publishers Ltd, pp 127-134.

Wendler D. Minimal risk in pediatric research as a function of age. Arch Pediatr Adolesc Med (2009) 163(2):115-8.

Wendler D and Emanuel E. What is a "Minor" Increase over Minimal Risk? J Pediatrics (2005) 147(5):575-578.

Wendler D and Varma S. Minimal risk in pediatric research. J Pediatr (2006) 149:855-861.

Wert $G$ de. Ethics of intracytoplasmic sperm injection: proceed with care. Hum Reprod (1998a) 13(Suppl 1):219-227.

Wert $G$ de. The post-menopause: playground for reproductive technology? Some ethical reflections. In: The Future of Human Reproduction. Ethics, Choice and Regulation-Harris J, Holm S, eds. (1998b) Oxford: Clarendon Press, pp 221-237.

Wert $\mathrm{G}$ de. Met het oog op de toekomst. Voortplantingstechnologie, erfelijkheidsonderzoek en ethiek. (1999) Amsterdam. Thela Thesis [in Dutch]

Wert $G$ de. Human cloning: the case of the (preimplantation) embryo, and ethical exploration. In: Assisted Conception. Research, Ethics and Law-Gunning J, ed. (2000) Ashgate Dartmouth: Aldershot, etc, pp 83-07.

Wert G de. Humane embryonale stamcellen als Heilige Graal. Een ethische reflectie. Filosofie \& Praktijk (2001) 22:34-56 [in Dutch].

Wert $\mathrm{G}$ de. Ethical aspects of prenatal testing and preimplantation genetic diagnosis for late-onset neurogenetic disease: the case of Huntington's disease. In: Prenatal Testing for Late-Onset Neurogenetic Disease-Evers-Kiebooms G, Zoeteweij MW, Harper PS, eds. (2002) Oxford: BIOS Scientific Publishers Ltd, pp 129-157.

Wert $G$ de. Handelingen met geslachtscellen en embryo's. Gezondheidsraad, Signalering Ethiek \& Gezondheid 2003 (2003) 11-50 [in Dutch] 
Wert G de. Preimplantation genetic diagnosis: the ethics of intermediate cases. Hum Reprod (2005) 20:3261-3266.

Wert $G$ de, Liebaers I, van de Velde $\mathrm{H}$. The future (r)evolution of preimplantation genetic diagnosis/human leukocyte antigen testing: ethical reflections. Stem Cells (2007) 25:2167-2172.

Walters LR and Palmer JG. The Ethics of Human Gene Therapy (1997) New York and Oxford: Oxford University Press.

Wertz DC, Fletcher JC, Berg K. Review of Ethical Issues in Medical Genetics (2003) Human Genetics Programme, World Health Organization.

White SL, Shanske S, Biros I, Warwick L, Dahl HM, Thorburn DR, DiMauro S. Two cases of prenatal analysis for the pathogenic $T$ to $G$ substitution at nucleotide 8993 in mitochondrial DNA. Prenat Diagn (1999a) 19:1165-1168.

White SL, Collins VR, Wolfe R, Cleary MA, Shanske S, DiMauro S, Dahl HM, Thorburn DR. Genetic counseling and prenatal diagnosis for the mitochondrial DNA mutations at nucleotide 8993. Am J Hum Genet (1999b) 65:474-482.

White SL, Shanske S, McGill JJ, Mountain H, Geraghty MT, DiMauro S, Dahl HH, Thorburn DR. Mitochondrial DNA mutations at nucleotide 8993 show a lack of tissue or age-related variation. J Inherit Metab Dis (1999c) 22:899-914.

Wivel NA and Walters L. Human germ-line gene therapy: the case of its development and use. J Med and Phil (1993) 14:593-612.

World Health Organisation. Safe Abortion: Technical and Policy Guidance for Health Systems (2003) Geneva: World Health Organisation.

World Medical Association. Declaration of Helsinki. Ethical Principles for Medical Research Involving Human Subjects (1964) Amendment $59^{\text {th }}$ WMA General Assembly, Seoul 2008.

Zhang J, Zhuang GL, Zeng Y, Acosta C, Shu YM, Grifo J. Pregnancy Derived From Human Nuclear Transfer. Fertil Steril (2003) 80:S56.

Zoeteweij MW, Nys K, Harper PS, Simpson SA, Dürr A, Jacopini G, Yapijakis C, Kieboom GE. An overview of prenatal testing for Huntington's disease in six European countries. In: Prenatal Testing for Late-Onset Neurogenetic DiseaseEvers-Kiebooms G, Zoeteweij MW, Harper PS, eds. (2002) Oxford: BIOS Scientific Publishers Ltd, pp 25-43.

Zoon KC. Letter to sponsors/researchers-human cells used in therapy involving the transfer of genetic material by means other than the union of gamete nuclei (2001) www.fda.gov/cber/ltr/cytotrans070601.htm.

Zuuren van F, Schie van EC, Baaren van NK. Uncertainty in the information provided during genetic counselling. Patient Educ Couns (1997) 32:129-139. 
Summary 

Whereas assisted reproductive technology (ART) was initially used for infertility problems, it has increasingly been deployed as a vehicle for genetic technology. This thesis examines ethical issues that arise at the interface of ART and genetics. More in particular, this thesis examines a specific case: the ethical issues that arise when reproductive genetic technology is used to prevent the transmission of mitochondrial disorders, particularly those caused by a mutation in the mitochondrial DNA (mtDNA). Whereas the ethical issues at the crossroads of ART and genetics are complex, this is even more so when reproductive genetic technology is deployed in the context of disorders caused by a mutation in the mtDNA.

When people talk about genes, they usually refer to the nuclear genome. All human cells, however, contain two different genomes. One indeed is located in the nucleus and follows a Mendelian pattern of autosomal recessive, dominant or X-linked inheritance. This genome is by far the largest, containing about $22.000-24.000$ genes. The other genome is located in the mitochondria. These are tiny organelles, which exist outside the nucleus, in the cytoplasm. They serve as the 'powerhouses' of the cell. The mitochondrial genome is extremely small, containing 37 genes, which is about $0.1 \%$ of the total amount of DNA. Mitochondrial DNA disorders are clinically heterogeneous but usually severe disorders, involving defects in energy production. As there is no curative treatment, helping carriers of mitochondrial mutations to have healthy children has been a central focus of attention. This thesis offers an ethical evaluation of four reproductive genetic technologies used with the aim of preventing the transmission of mtDNA disease: prenatal diagnosis (PND), preimplantation genetic diagnosis (PGD), ooplasmic transfer (OT) and mitochondrial gene replacement by means of nuclear transfer (NT). Addressed are both the ethical issues 'at the bench', when a technology is preclinically (in the laboratory) developed as well as the ethical issues 'at the bedside', when a technology is offered to patients in the clinic.

PND and PGD involve genetic testing and selection of embryos and foetuses. PND refers to the genetic testing of a fetus, either by means of chorionic villus sampling (around the $12^{\text {th }}$ week of gestation), or by means of amniocentesis (week 14-18 of gestation), possibly followed by a termination of pregnancy. In case of PGD, embryos created by means of in vitro fertilization (IVF) are analysed for a particular genetic mutation. After the genetic analysis, one or two unaffected embryos are transferred to the womb.

OT and NT involve genetic intervention or modification. OT encompasses the transfer of donor ooplasm with normal mitochondria to the oocyte of a woman who 
carries mutant mtDNA in her oocytes. NT involves the transfer of the nuclear DNA of a woman carrying an mtDNA mutation into an enucleated donor oocyte. This should result in healthy offspring carrying the mtDNA of the oocyte donor and the nuclear genome of the prospective parents. The nuclear transplantation can be performed before and after IVF, using the nucleus of an unfertilized oocyte, the pronuclei of the zygote or the nucleus of a blastomere of an embryo.

Chapter 2 offers an overview of the moral landscape of PND and PGD for mitochondrial disorders. The terms 'mitochondrial disorders', 'mitochondrial DNA disorders' and 'mitochondrial mutations' are sometimes interchangeable used. Strictly speaking, however, these are separate categories, raising separate ethical questions. Therefore, we made a further classification of the different concepts. An ethically relevant distinction exists between applying ART for carriers of mitochondrial disease caused by a defect of the nuclear genome and for carriers of mtDNA mutations. The first group of mutations shows a Mendelian inheritance pattern. Offering ART, then, is comparable with 'standard' applications of ART. This is not to say that these applications are ethically unproblematic, but they do not raise novel ethical questions.

When applying ART for the second group of mitochondrial disorders, the 'mtDNA disorders', however, the ethical issues are intensified. Mitochondrial genetics varies from Mendelian genetics in several aspects. As a consequence, diseases due to a defect in the mtDNA show some special characteristics. Firstly, many mtDNA mutations are heteroplasmic. This means that there is a mixture of normal and mutant mtDNA in one person, the level of which can differ among tissues. If the mutant load, i.e. the ratio of mutant to normal mtDNA, exceeds a specific threshold, clinical features become manifest. However, the exact threshold to disease expression is often not known and genotype-phenotype correlations are often lacking, even within families. Secondly, the mtDNA is maternally inherited. Due to the so-called genetic bottleneck, extreme shifts in mutant load can be observed between mother and child and also siblings can have variable levels of mutant load. The characteristics of mtDNA make it difficult to estimate recurrence risks for a couple and if one decides to conduct PND or PGD, it is due to heteroplasmy often very difficult to interpret the test results and to predict the phenotype of the child. A limited number of mtDNA mutations allow reliable predictions, though results in the 'grey zone' are problematic. As a consequence, we show that these applications of PND and PGD confront both clinical practice and society at large with several ethical questions and issues for further debate, among which the acceptability of suboptimal genetic testing, the value and research use of embryos, terminating a pregnancy based on ambiguous data, the evaluation of late abortion, the ethics of PGD for 
disorders with an incomplete penetrance and variable expression, the possible transfer of embryos with residual health risks, the acceptability of risks and drawbacks of genetic reproductive technology in general, and the scope and limits of reproductive autonomy and professional responsibility.

Chapter 3 goes more deeply into the subject of PGD for mtDNA disorders. Traditional applications of PGD are targeted at Mendelian and chromosomal disorders. In those cases, it is in principle certain whether the relevant gene defect is present and which of the available embryos can safely be transferred. In case of PGD for mtDNA disorders, this is less clear. It is conceivable that in some cycles only affected embryos are available for transfer. So instead of 'promising' parents a healthy child, PGD in these cases can only aim at reducing reproductive risk. From an ethical point of view, such a 'risk-reducing' PGD raises challenging questions about parental and medical responsibilities. The main argument in favour of PGD is that it offers couples at risk the opportunity of reducing their chances of having a severely affected child. Another important argument in favour of PGD concerns reproductive autonomy. This is usually defined as the right to control one's own procreation unless there is a compelling reason for denying a person that control. The rationale behind this principle is that in a liberal democratic society, the presumption is that people should be free to make their own choices according to their own values.

There are many possible arguments against such a 'risk-reducing' PGD. For example, one could argue that the transfer of an embryo at risk is at odds with the welfare of the future child. We defended the reasonable welfare standard: (medically assisted) reproduction is morally acceptable as long as the resulting child has a reasonable chance of an acceptable quality of life; a high risk of serious harm would be the contra-indication. From this perspective, the transfer of an embryo at risk may be justifiable as long as the child will not run a high risk of serious harm. Another possible objection to PGD for mtDNA disorders is that such an application of PGD is too complex for parents too decide - and also our empirical studies show that many professionals consider mitochondrial genetics a 'test case for reproductive autonomy'. Nevertheless, to deprive competent couples from reproductive options because the decisions are too complex amounts to a form of paternalism that would be hard to justify.

Although potential objections are manifold, we conclude that none of them supplies convincing moral arguments to regard risk-reducing $P G D$ as unacceptable. Nevertheless, introducing this new application of PGD in clinical practice will raise further complex issues of determining conditions for its responsible use. 
In chapter 4 we examine the ethical questions that may successively arise when a couple asks for PGD for an mtDNA mutations and elaborate which conditions should be met in order to justifiably introduce PGD for mtDNA disorders in clinical practice. We defended two starting points. The first starting point regards the reasonable welfare standard (the child conceived by means of PGD for mtDNA disorders should have a reasonable chance of an acceptable quality of life). Using this standard has several implications. It for example implies that before PGD for mtDNA disorders can be considered, it should be clear whether PGD is sufficiently reliable. It is important to ascertain that the cell taken for analysis adequately represent the composition of the embryo as a whole. It also implies that an appropriate cut-off point is determined. A cut-off point is the threshold of mutant load above which no embryos are considered for transfer - and below which embryos are eligible for transfer. We scrutinize how to determine these cut-off points and how to use them in clinical practice - for example, when parents ask to take more or less risks. By avoiding a high risk of serious harm, the reasonable welfare standard formulates the lower limit of medical acting. If however, other things being equal, it is reasonably possible to reduce the risks of a medical treatment (or to enhance its benefits), ART included, then that is of course what the medical team should aim at. This is the rationale of the second starting point: continuous efforts should be made to minimize the risks as much as reasonably possible. This implies that although residual risks may well be acceptable, the parents and the medical team should make efforts to search for the 'best possible' embryo, meaning the embryo with the lowest mutant load and morphologically sound. We discuss several possibilities for searching for 'better' embryos, such as generating more embryos or starting a new IVF/PGD cycle.

Finally, we discuss whether follow-up studies should be conducted after the IVF/PGD procedure. Genetic testing of children born as a result of IVF/PGD for an mtDNA mutation could validate the PGD procedure by ascertaining that the genetic diagnosis made at the embryonic stage is a reliable predictor of the genotype and phenotype of the resulting child. This type of genetic testing would entail non-therapeutic medical scientific research with minors and is morally complex. A possible tension exists between the need to ensure that PGD is safe, effective and reliable and the need to protect the participating children. After weighing the many ethical pitfalls of genetically testing minors, we recommend to consider conducting a genetic test after birth.

Chapter 5 reports the results of the interviews that have been conducted with researchers and clinicians. Whereas a scientific and ethical debate about the possible reproductive options for carriers of mtDNA disease is developing, not much 
information regarding the views and experiences of professionals exists. The results of our empirical study illustrate the impact on professionals of reproductive decisionmaking in the context of mtDNA disease. Professionals struggle with the handling of ambiguous data often linked to PND and PGD for mtDNA disorders, and all professionals feel that the inherent challenges of reproductive genetic testing and counseling are intensified in the context of mtDNA disorders. Professionals tend to regard the uncertainties as conflicting with their task of providing accurate information when counseling couples. They struggle with the tension between on the one hand the ideal of reproductive autonomy and on the other hand the reality of complex clinical decision-making. Whereas part of the professionals hold the opinion that reproductive autonomy is not endangered, others doubt whether the nuances of mitochondrial genetics can still be explained. Some have therefore decided not to offer specific types of prenatal tests or they exclude testing for some mutations. Those more positive towards testing introduce several mechanisms to control and reduce the uncertainties. They for example emphasize that only very experienced and well-equipped professionals in leading centers should offer this type of testing. Furthermore, those professionals are very anxious to find out the precise risks and to communicate these to their patients.

Although knowing the magnitude of any remaining risks is necessary for determining whether testing can be justifiably offered and although it is interesting from a scientific point of view, more precise calculations will not always provide couples involved with a better basis for decision-making. Besides the fact that people have a poor understanding of quantitative risk information, it can be questioned whether knowing the risks in detail will be helpful for them. Instead, professionals could pay attention to the consequences of living with a child with a mtDNA disorder, the range of possible clinical manifestations, the expected quality of life and the possibilities and limitations for the child (and its siblings). Here, the role of the counselor is to help people see what the possible consequences of the different reproductive options are. This implies the counselor and couple should discuss the uncertainties and moral dilemmas involved, the different reproductive strategies and their pros and cons. This chapter may be perceived as an example of how professionals deal with the inherent limitations in genetic knowledge representing the state of the art. After all, novel developments in medical genetics will continuously confront professionals with ambiguous information. Part of the uncertainty in mitochondrial genetics may be caused by insufficient knowledge and may therefore be reduced by future research. However, part of the uncertainty may be inherently linked to mtDNA genetics. In that case, reproductive counseling and decision-making will always remain complex in the context of mtDNA disease. 
Chapter 6 identifies the ethical issues involved in OT and NT for mtDNA mutations, both the ethical issues associated with the development of OT and NT in the laboratory as well as ethical issues associated with a possible clinical application. Furthermore, we distinguish the conceptual issues from the ethical issues. The first conceptual issue regards the question whether OT and NT should be classified as forms of germ-line modification. The second conceptual issue regards the question whether NT is a type of human cloning. The ethical issues associated with OT and NT for mtDNA disease are manifold. For example, one could argue that NT leads to a child with two genetic mothers, one providing the nuclear DNA, the other the mtDNA. This provokes a discussion about the moral implication of 'three genetic parents' and the social meaning of genetic parenthood. Other ethical questions regard the acceptability of oocyte donation: oocytes are needed both for research and for reproductive purposes. Another major point of concern regards the health and safety risks for the child conceived by means of NT.

Whereas chapter 6 mapped the moral landscape of OT and NT for mtDNA disorders, chapter 7 deepens the ethical issues involved with germ-line modification and reproductive cloning. First, we discuss whether NT entails germ-line modification. The mitochondrial genome differs in many aspects from the nuclear genome: first, the mitochondrial genome contains only a tiny amount of the total amount of DNA; second, it has a very specific function (providing the cell's energy metabolism); third, it has its own transmission pattern. However, the distinctive feature of germ-line modification is that the genetic modification can be transmitted to further generations. Therefore, there is no good reason for not also applying the concept of germ-line modification to modification of the mitochondrial genome. Second, we discuss whether NT entails reproductive cloning. The answer to this question also depends on which definition of cloning one deploys. One variant of NT, in which the nucleus of a blastomere is transferred to an enucleated oocyte, may indeed amount to reproductive cloning. More in particular, it may amount to reproductive embryo cloning. If one deploys a 'broad' definition, then reproductive cloning refers to genetic copying - in our case the copying of an embryo. If one deploys a 'narrow' definition, then genetic copying is necessary but not sufficient; reproductive cloning, then, also presupposes genetic identity with at least one other born individual. In that case, transferring the nucleus of a blastomere to a donor oocyte would only involve reproductive cloning as long as at least two identical embryos are thus made, transferred and carried to term.

Subsequently, we examine the validity of the main categorical (non-safety) arguments used against germ-line modification and reproductive cloning. Opponents have for instance argued that germ-line modification and reproductive cloning are 
repugnant and unnatural, or that they violate human dignity. Others have argued that germ-line modification would violate the child's right to an open future. This means a right to have one's future options kept open until one is capable of making one's own decisions. The moot point of the argument is the fear that it would become possible to alter presumed 'essential characteristics' of the make up of future generations. For this reason, germ-line modification of the nuclear DNA has led to more ethical controversy than modification of the mtDNA. We challenge the assumption that modification of the mtDNA is ethically less problematic than modification of the nuclear DNA because the first would not involve the possibility of altering essential characteristics. Even if mtDNA has only a basic cellular function, then it is still meaningful to say that germ-line modification of the mtDNA is likely to change the identity of the future person. However, even if NT may alter the identity of the child, then a clinical application of NT to prevent mtDNA, so we argue, could still be compatible with the position that one should not violate the child's right to an open future.

We conclude that were NT for mtDNA disease to become safe and effective, dismissing it because it involves germ-line modification and possibly also reproductive cloning is unjustified.

In chapter 8, we examine the moral acceptability of adding sex selection to PGD and/or NT for mtDNA disorders. A recent study has shown that a specific variant of NT is feasible in non-human primates. Due to this technical breakthrough, it seems only a matter of time before a first-in human application is considered. The health and safety risks for the child conceived by means of NT, however, are a major point of concern. In a clinical application of NT it may be difficult to avoid small amounts of affected mitochondria coming along with the oocyte, the pronuclei or the nucleus of the recipient woman. Although it is unlikely that this small amount of mutant mtDNA leads to clinical disease symptoms in the resulting child, it may rise to clinically relevant amounts in a next generation. This is also relevant when in case of PGD a low mutant low embryo is transferred. Due to the existence of a genetic bottleneck, a low-level mutant load in the second generation (the child to be conceived through PGD or NT) may rise to higher levels in the third generation (the couple's grandchildren). As mitochondria are transferred maternally, male offspring will not pass on their mutant DNA to the next generation. This leads to the question whether the avoidance of transgenerational health risks provides a morally acceptable reason for sex selection.

We discuss post- and preconceptional sex selection strategies. With postconceptional sex selection we refer to sex selection of IVF-embryos, which 
requires PGD followed by selective transfer of a male embryo. With preconceptional sex selection we refer to sex selection prior to fertilization, which requires separation of X-bearing and Y-bearing spermatozoa, with subsequent selection for artificial insemination or IVF. We argue that adding sex selection for this purpose is morally acceptable in principle. Even though the health of the child to be conceived is not at stake (as required in the traditional justification of sex selection for medical reasons), the reason for choosing or preferring a boy is still health-related. However, the proportionality of adding post- and/or preconceptional sex selection to PGD or NT for mtDNA disease is a relevant concern. If PGD is already part of the procedure, either as the central technology or as a back-up test after NT, preferentially transferring male embryos could be a morally acceptable way of reducing possible risks and burdens. To start an IVF/PGD-cycle especially for this purpose would be disproportional. In theory, preconceptional sex selection is a morally superior option: it is less intrusive, less costly and it does not involve embryo loss. In practice, however, preconceptional sex selection is not fail-safe, especially not for selecting $Y$ bearing sperm. Nevertheless, preconceptional sex selection may be morally justified as a means to increase the chances of obtaining male embryos.

Chapter 9 looks back at the main results, conclusions and limitations of this thesis and forward to questions for future research. We conclude that all strategies, with the exception of OT, could potentially serve as acceptable reproductive options for couples at risk of transmitting an mtDNA mutation to their future offspring. As OT is unsuitable as a reproductive option for carriers of mtDNA mutations and not proven safe, clinically offering OT would be both futile and unethical. With regard to PND, PGD and NT, the adjective 'potentially' is used to indicate that none of these strategies is unconditionally acceptable. All reproductive options ask for a further filling in: whereas PND and PGD require further qualifications for a responsible use in clinical practice, NT requires further preclinical research before a clinical application could be appropriate (and the ethics of a first-in-human application of NT for mtDNA asks for further debate as well). Having to reproduce by means of one of the reproductive interventions as described in this thesis is, in some way, a tragic situation as all interventions offer some serious moral disadvantages. If, or as long as, not one strategy clearly towers above the others, it seems reasonable to allow the couple to prioritise and balance the pros and cons of the available interventions according to their own values (and these are considerations in which reasonable people may come to different conclusions). Of course, this is not without any qualifications. After all, the moral minimum as defended in this thesis regards the avoidance of a high risk of serious harm for the child, added with continuous efforts to reduce these risks as much as reasonably possible. 
Samenvatting 



\section{Ethiek op het snijvlak van voortplantingsgeneeskunde en genetische technologie: mitochondriële ziekten als casus}

Hoewel aanvankelijk ontwikkeld om mensen met vruchtbaarheidsproblemen te helpen kinderen te krijgen, wordt voortplantingstechnologie steeds vaker ook gebruikt als 'kruiwagen' voor genetische technologie. Technieken op het snijvlak van voortplantingsgeneeskunde en genetica, 'reproductieve genetische technologie', roepen complexe ethische vraagstukken op. Dit geldt des te meer wanneer deze technologie wordt gebruikt ter preventie van ziekten die veroorzaakt worden door een mutatie (wijziging) in het mitochondrieel DNA (mtDNA), de zogenaamde 'mitochondriële DNA-ziekten'.

Wanneer mensen over genen praten, bedoelen ze daar meestal onze kerngenen mee, oftewel het kern-DNA. Alle menselijke cellen bevatten echter twee verschillende soorten DNA. Eén daarvan is gelegen in de celkern en volgt een Mendeliaans overervingspatroon, waarbij de genen voor de ene helft via de moeder en voor de andere helft via de vader worden geërfd. Met ongeveer 22.000 tot 24.000 genen is dit verreweg het grootste genoom.

Het ander genoom, dat uitsluitend via de moeder wordt doorgegeven, ligt in de mitochondriën. Deze kleine organellen liggen buiten de celkern, in het cytoplasma van de cel. Het zijn de 'energiefabriekjes' van de cel. Met slechts 37 genen is het mitochondrieel genoom heel klein - het maakt slechts $0.1 \%$ van ons totale DNA uit. Mutaties in het mtDNA kunnen echter leiden tot een breed scala aan veelal ernstige ziekten, meestal in organen of weefsels die veel energie nodig hebben. Patiënten melden zich in de kliniek met uiteenlopende aandoeningen, waaronder spierziekten, lever- en hartfalen, diabetes, blindheid, doofheid en neurologische aandoeningen. Een goede behandeling is er vaak niet. Daarom proberen draagsters van mitochondriële mutaties veelal te voorkomen dat ze de mutatie doorgeven aan hun nageslacht. In theorie hebben stellen met een kinderwens vier mogelijkheden om te proberen gezonde, genetisch eigen kinderen te krijgen: prenatale diagnostiek, preimplantatie genetische diagnostiek (PGD), ooplasmatransplantatie (OT) en celkerntransplantatie (in dit proefschrift 'nuclear transfer', NT, genoemd). Dit proefschrift biedt een ethische evaluatie van het gebruik van deze reproductieve genetische technologieën ter preventie van mtDNA-ziekten. Daarbij wordt zowel aandacht besteed aan ethische vragen die rijzen in het laboratorium, wanneer een techniek ontwikkeld wordt, als aan ethische vragen die zich voordoen aan het bed, wanneer een techniek in de kliniek wordt aangeboden.

Prenatale diagnostiek is het genetisch testen van een foetus door middel van een vlokkentest (rond de twaalfde week van de zwangerschap) of vruchtwaterpunctie 
(week veertien tot achtien). Bij aanwezigheid van de gezochte mutatie kunnen ouders overwegen de zwangerschap af te breken.

Bij PGD (ook wel embryoselectie genoemd) worden door middel van in vitro fertilisatie (IVF) gemaakte embryo's onderzocht op de specifieke genetische mutatie. Dit gebeurt meestal in het achtcellig stadium. Gezonde embryo's komen vervolgens in aanmerking voor plaatsing in de baarmoeder, met als doel de totstandbrenging van een zwangerschap.

In geval van OT wordt ooplasma van een gedoneerde eicel, zonder de celkern maar met mitochondriën, in de eicel van een draagster van een mtDNA-mutatie geïnjecteerd.

In geval van NT wordt het kern-DNA van een draagster van een mtDNA-mutatie getransplanteerd naar een 'ontkernde' donoreicel (dat wil zeggen dat de kern uit de eicel is gehaald). De nieuwe eicel die daardoor ontstaat heeft het kern-DNA van de wensmoeder en de mitochondriën (met het mtDNA) van de eiceldonor. De transplantatie kan zowel voor als na de bevruchting (door middel van IVF) plaatsvinden, dus bij de onbevruchte en bij de bevruchte eicel (embryo).

In hoofdstuk 2 wordt het morele landschap van prenatale diagnostiek en PGD voor mitochondriële ziekten in kaart gebracht. De termen mitochondriële ziekten, mitochondrieel DNA ziekten en mitochondriële mutaties worden vaak door elkaar gebruikt. Strikt gesproken zijn het echter verschillende categorieën, met verschillende ethische vragen. Daarom wordt in dit hoofdstuk een verdere classificatie van de verschillende concepten en de daarmee gepaard gaande ethische vragen gemaakt. Er bestaat een ethisch relevant onderscheid tussen het gebruik van reproductieve genetische technologie voor draagsters van mitochondriële ziekten die veroorzaakt worden door een mutatie in de kerngenen en voor draagsters van mtDNA-mutaties. Het gebruik van reproductieve genetische technologie ter voorkoming van het doorgeven aan het nageslacht van de eerste groep mutaties is vergelijkbaar met de 'standaard' toepassingen van diezelfde technologie in de voortplantingsgeneeskunde. Dit betekent niet dat deze toepassingen ethisch onproblematisch zijn, maar zij geven geen aanleiding tot nieuwe ethische vragen. Wanneer reproductieve genetische technologie gebruikt wordt voor hulp bij voortplanting aan draagsters van een mutatie in de tweede groep, de mtDNA-mutaties, dringen zich nieuwe ethische vragen op. Dit komt door de specifieke kenmerken van de mitochondriële genetica.

Ten eerste zijn veel mtDNA-mutaties heteroplasmisch. Heteroplasmie wil zeggen dat er in één individu zowel gemuteerd als normaal mtDNA is, waarbij een hele reeks aan verhoudingen tussen afwijkend en normaal mtDNA mogelijk is. Als de 'mutant load', dat wil zeggen de verhouding tussen gemuteerd en normaal mtDNA een 
bepaalde drempel overschrijdt, kunnen ziektesymptomen optreden. De exacte drempel is echter vaak niet bekend. Ten tweede vindt de overerving van het mtDNA plaats langs maternale lijn: kinderen erven hun mitochondriën van hun moeder en derhalve geven alleen dochters het mtDNA weer door aan de volgende generatie. Door het mechanisme van de genetische flessenhals (genetic bottleneck) kunnen tussen generaties grote verschuivingen in mutant load optreden. Variatie in de ernst van de ziekte door verschillen in de verhouding tussen gemuteerd en normaal mtDNA komen zelfs binnen families en tussen broers en zussen voor.

Door deze kenmerken is het moeilijk om voor dragers met kinderwens de risico's op een aangedaan kind in te schatten. Als een stel met behulp van prenatale diagnostiek of PGD een kind probeert te krijgen, is het door heteroplasmie vaak moeilijk om de resultaten van de genetische test te interpreteren en de gezondheid van het toekomstig kind te voorspellen. Voor slechts een beperkt aantal mtDNAmutaties zijn betrouwbare voorspellingen te doen, en zelfs voor deze mutaties zijn testuitslagen in de tussenliggende grijze gebieden problematisch. Als gevolg hiervan roepen zowel prenatale diagnostiek en PGD voor mtDNA mutaties een grote variëteit aan ethische vragen op. Mogen suboptimale genetische tests worden aangeboden? Mogen embryo's gebruikt (en gekweekt) worden voor wetenschappelijk onderzoek? Is het geoorloofd om embryo's met mogelijke gezondheidsrisico's in de baarmoeder te plaatsen? Is het aanvaardbaar om een zwangerschap af te breken op grond van ambivalente informatie, en zo ja, tot wanneer in de zwangerschap mag dat? Een andere vraag betreft de ethiek van PGD voor ziekten met een incomplete penetrantie en variabele expressie (dat wil zeggen dat niet iedereen met de mutatie ziek wordt en dat een variabiliteit in ernst en verschijnselen van de ziekte bestaat). Relevante overwegingen zijn ook de reikwijdte van het principe van reproductieve autonomie, de professionele verantwoordelijkheid van artsen en de aanvaardbaarheid van risicovolle voortplantingstechnologie.

In hoofdstuk 3 wordt dieper ingegaan op de ethische aanvaardbaarheid van PGD voor mtDNA-ziekten. Traditionele toepassingen van PGD zijn gericht op Mendeliaanse en chromosomale ziekten, waarbij in principe duidelijk is of het gemuteerde gen in het embryo aanwezig is en welk embryo teruggeplaatst kan worden. Dit is minder duidelijk voor mtDNA-ziekten en het is goed denkbaar dat slechts aangedane embryo's voorhanden zijn. In deze gevallen kan PGD het risico op een aangedaan kind wel verminderen, maar niet wegnemen. Het belangrijkste argument vóór een dergelijke risico-reducerende PGD is dat het stellen met een kinderwens de mogelijkheid biedt het risico op een ernstig aangedaan kind te verminderen en de hogere risico's van een natuurlijke voortplanting te omzeilen. Een ander belangrijk argument voor PGD is het principe van reproductieve autonomie: de 
vrijheid van mensen om zelf te beslissen óf ze kinderen willen, en zo ja hoe, wanneer, hoeveel, met wie en onder welke omstandigheden. De overheid grijpt alleen in als daar dwingende redenen voor zijn. De gedachte achter dit principe is dat in een liberaal-democratische maatschappij, waarin verschillende opvattingen over het goede leven bestaan, mensen zo veel mogelijk vrijheid moet worden gegeven om hun eigen beslissingen te nemen volgens de waarden die voor hen belangrijk zijn juist ook bij hoogstpersoonlijke zaken als het al dan niet krijgen van kinderen.

Er zijn verschillende argumenten tégen een dergelijke risico-reducerende PGD in te brengen. Zo is bijvoorbeeld te betogen dat het in de baarmoeder plaatsen van een embryo als sprake is van een bekend risico dat deze zal uitgroeien tot een kind dat later ziek wordt, niet in overeenstemming is met de verantwoordelijkheid van de arts om rekening te houden met het welzijn van het toekomstig kind. In dit proefschrift wordt de standaard verdedigd dat (medisch geassisteerde) voortplanting moreel aanvaardbaar is zolang het resulterende kind een redelijke kans heeft op een acceptabele kwaliteit van leven; de contra-indicatie is een 'hoog risico op ernstige schade'. We noemen dit de 'redelijke welzijnsstandaard' (reasonable welfare standard). Vanuit dit perspectief is het terugplaatsen van een embryo aanvaardbaar zolang de gezondheidsrisico's niet zo groot zijn dat het toekomstig kind een grote kans heeft om ernstig aangedaan te zijn. Ook al is PGD voor mtDNA-ziekten niet zonder bezwaren, geen van deze bezwaren vormt een doorslaggevend argument om deze (nieuwe) toepassing van PGD op voorhand af te wijzen. Wel zal de introductie in de kliniek aanleiding geven tot een reeks complexe ethische vragen en er zullen dan ook voorwaarden geformuleerd moeten worden voor een verantwoord gebruik in de kliniek.

In hoofdstuk 4 bespreken we de ethische vragen die rijzen wanneer een stel daadwerkelijk bij de kliniek aanklopt voor PGD voor een mtDNA mutatie. We formuleren de voorwaarden en zorgvuldigheidsvereisten waaraan voldaan moet zijn wil PGD voor een mtDNA-mutatie ethisch te verantwoorden zijn. We verdedigen hierbij twee uitgangspunten, die beide consequenties hebben voor het ontwikkelen en aanbieden van PGD voor mtDNA-ziekten.

Het eerste uitgangspunt, de redelijke welzijnsstandaard, impliceert dat van tevoren duidelijk moet zijn dat PGD voor de betreffende mutatie voldoende betrouwbaar is; er dient vastgesteld te worden dat de geanalyseerde cel representatief is voor de overige cellen van het embryo. Een andere implicatie van de redelijk welzijnsstandaard is dat van tevoren een drempelwaarde (cut-off point) moet worden vastgesteld. Embryo's met een mutant load boven deze drempelwaarde komen niet in aanmerking voor terugplaatsing. Vervolgens bespreken we hoe een dergelijke drempelwaarde per mutatie vastgesteld kan worden en hoe deze vervolgens 
gehanteerd kan worden in de kliniek - bijvoorbeeld wanneer een stel vraagt meer of juist minder risico's te nemen.

Het voorkómen van een hoog risico op ernstige schade zien we als de morele ondergrens van het medisch handelen. Daarnaast verdedigen we als tweede uitgangspunt de morele inspanningsverplichting om de risico's van een medische behandeling zoveel als redelijkerwijs mogelijk te verminderen. Dit impliceert dat hoewel restrisico's acceptabel zijn, toekomstige ouders en het medisch team zich tegelijkertijd moeten inspannen om het best mogelijke embryo te zoeken. Hiermee bedoelen we een kwalitatief goed embryo met een zo laag mogelijke mutant load. We bediscussiëren verschillende manieren waarop naar betere embryo's gezocht kan worden, bijvoorbeeld door het genereren van meer embryo's of door het opstarten van een nieuwe IVF/PGD-cyclus.

Tot slot bespreken we of, en zo ja welk follow-up onderzoek moet worden gedaan na het PGD-traject. Het genetisch testen van de kinderen geboren door middel van PGD voor een mtDNA-mutatie zou kunnen uitwijzen of PGD inderdaad betrouwbaar is voor de mutatie in kwestie. Het kan uitwijzen of de genetische diagnose in het embryonale stadium een goede voorspeller is van de genetische opmaak en de gezondheid van het kind na de geboorte. Een dergelijke test komt echter neer op het doen van niet-therapeutisch wetenschappelijk onderzoek met minderjarigen, een complex en gevoelig thema. Er bestaat een mogelijk spanningsveld tussen enerzijds de behoefte vast te stellen of PGD veilig, effectief en betrouwbaar is en anderzijds de bescherming van het kind. Ondanks de verschillende ethische valkuilen doen we de aanbeveling dat het genetisch testen van pasgeborenen tenminste moet worden besproken en overwogen.

In hoofdstuk 5 worden de resultaten van de interviews met onderzoekers en artsen gerapporteerd. Kenmerkend voor prenatale diagnostiek en PGD voor mtDNAmutaties is de mogelijk dubbelzinnige en onzekere informatie. Professionals worstelen hiermee: zij ervaren het reproductief genetisch testen en counselen in de context van mtDNA-ziekten als uitermate lastig. Ze vinden dat de onzekerheden soms conflicteren met hun taak om accurate informatie te verschaffen aan paren en worstelen met het spanningsveld tussen enerzijds het ideaal van reproductieve autonomie en anderzijds de complexe realiteit van de klinische besluitvorming. Een deel van de professionals is dan ook van mening dat de onzekerheden en technische details van de mitochondriële genetica een bedreiging vormen voor de autonome besluitvorming van paren. Zij betwijfelen of de nuances van de mitochondriële genetica nog wel uitgelegd kunnen worden. Sommigen hebben daarom besloten bepaalde prenatale tests niet (meer) aan te bieden voor mtDNA-mutaties, of dat voor bepaalde mtDNA-mutaties niet meer te doen. Diegenen die positiever tegenover 
reproductief genetisch testen staan, introduceren verschillende mechanismen om de onzekerheden te beheersen en te verminderen. Ze benadrukken bijvoorbeeld dat alleen zeer ervaren en goed geoutilleerde professionals in leidende centra deze tests mogen aanbieden. Voorts zijn deze professionals er op gebrand om de risico's heel precies te berekenen en in detail met hun patiënten te bespreken.

De grootte van de risico's is natuurlijk van belang om te bepalen of het ethisch verantwoord is om bepaalde genetische tests aan te bieden. Dat is ook van belang vanuit een wetenschappelijk perspectief. Echter, een heel precieze risicoberekening zal paren waarschijnlijk niet helpen bij het maken van beslissingen. Naast het gegeven dat mensen moeite hebben met het verwerken van kwantitatieve risicoinformatie, kan men zich afvragen of kennis van heel gedetailleerde risico's behulpzaam is bij het maken van reproductieve beslissingen. Daarom doen we verdere suggesties voor de counseling. Professionals zouden in de counseling vooral aandacht moeten besteden aan wat het wil zeggen om te leven met een kind met de desbetreffende mtDNA-ziekte, aan de aard van de mogelijke ziektesymptomen, aan de te verwachten kwaliteit van leven en aan de voor het kind te verwachten mogelijkheden en beperkingen. Het is de rol van de counselor mensen te helpen de mogelijke consequenties van de verschillende reproductieve opties te (over)zien. Dit betekent dat de counselor en het paar de onzekerheden, de morele dilemma's en de voors en tegens van de verschillende reproductieve strategieën met elkaar bespreken.

De empirische studie kan gezien worden als een illustratie van hoe professionals omgaan met de beperkingen en onzekerheden die inherent zijn aan toepassing van de nieuwste wetenschappelijke kennis. Nieuwe ontwikkelingen in de medische genetica zullen professionals voortdurend confronteren met dubbelzinnige informatie. Voor zover de onzekerheden waarmee de toepassing van de mitochondriële genetica gepaard gaat, voortkomen uit een gebrek aan kennis, kan wetenschappelijk onderzoek die onzekerheden verminderen. Voor zover het echter gaat om de inherente complexiteit van de mitochondriële genetica zal een restonzekerheid (en daarmee een ingewikkeld counseling- en besluitvormingsproces) onvermijdelijk blijven.

In hoofdstuk 6 wordt het morele landschap van OT en NT voor mtDNA-ziekten in kaart gebracht. We maken een onderscheid tussen conceptuele en normatieve vraagstukken. Een eerste conceptueel punt is of OT en NT vormen van kiembaanmodificatie zijn. Een tweede conceptueel punt is of NT een vorm van kloneren is. Daarna inventariseren we de normatieve vragen. NT resulteert bijvoorbeeld in een kind met genetisch materiaal van twee vrouwen, van wie één het kern-DNA doorgeeft en één het mtDNA. Dit leidt tot een discussie over de morele 
implicaties van het hebben van drie genetische ouders en de maatschappelijke betekenis van genetisch ouderschap. Een andere ethische vraag betreft de aanvaardbaarheid van eiceldonatie: zowel voor onderzoeksdoeleinden als voor het tot stand brengen van een zwangerschap zijn gedoneerde eicellen nodig. Ook de mogelijke gezondheidsrisico's voor het toekomstig kind waarmee de toepassing van OT en NT gepaard kan gaan zijn een belangrijk punt van aandacht.

In hoofdstuk 7 wordt dieper ingegaan op twee specifieke ethische aspecten van OT en NT: kiembaanmodificatie en reproductief kloneren. De discussie over kiembaanmodificatie heeft zich vooral toegespits op wijziging van het kern-DNA. In een aantal opzichten verschilt het mtDNA van het kern-DNA. Ten eerste maakt het mtDNA slechts een klein onderdeel uit van het totale DNA van een mens. Ten tweede heeft het mtDNA een heel specifieke functie (de cel van energie voorzien). Ten derde heeft het mtDNA haar eigen overervingmechanisme. We beargumenteren dat NT voor mtDNA-ziekten ondanks deze verschillen toch als een vorm van kiembaanmodificatie moet worden gezien. Kenmerkend voor kiembaanmodificatie is immers dat er een wijziging in het DNA wordt aangebracht die doorgegeven wordt aan volgende generaties. $\mathrm{Er}$ is daarom geen goede reden om alleen bij het aanbrengen van wijzigingen in het kern-DNA van kiembaanmodificatie te spreken en niet óók als het gaat om verandering van het mtDNA.

Hierna bespreken we of NT een vorm van reproductief kloneren is. Het antwoord op deze vraag hangt af van de definitie. Eén variant van NT, waarin de kern van een blastomeer (één cel uit een achtcellig embryo) in een ontkernde donoreicel wordt geplaatst, zou inderdaad als een vorm van reproductief kloneren kunnen worden gezien, meer in het bijzonder als een vorm van reproductieve embryoklonering. In een brede definitie verwijst reproductief kloneren naar genetisch kopiëren - in onze discussie: het tot stand brengen van genetisch identieke embryo's. In een smalle definitie is genetisch kopiëren noodzakelijk maar niet voldoende: voordat van reproductief kloneren gesproken kan worden dient er sprake te zijn van genetische identiteit met ten minste één geboren individu. Als die definitie wordt gevolgd zou het transplanteren van een kern van een blastomeer naar een gedoneerde eicel alleen reproductief kloneren behelzen wanneer dit resulteert in de geboorte van ten minste twee genetisch identieke kinderen.

Hierna onderzoeken we de validiteit van de belangrijkste argumenten tegen kiembaanmodificatie en reproductief kloneren. We gaan hier in op de categorische bezwaren, niet op de gezondheidsrisico's en veiligheidsaspecten. Tegenstanders zeggen bijvoorbeeld dat kiembaanmodificatie en reproductief kloneren weerzinwekkend en onnatuurlijk zijn, of dat ze de menselijke waardigheid aantasten. Anderen betogen dat kiembaanmodificatie het recht van een kind op een open 
toekomst schendt: we zouden de toekomst van kinderen zo open mogelijk moeten houden zodat ze, wanneer ze daartoe later zelf in staat zijn, hun eigen keuzes kunnen maken. De kern van dit argument is de vrees dat het mogelijk wordt essentiële kenmerken van toekomstige personen te veranderen. Mede om deze reden heeft kiembaanmodificatie van het kern-DNA tot meer ethische controverse geleid dan wijziging van het mtDNA. We betwisten de veronderstelling dat modificatie van het mtDNA ethisch minder problematisch is dan wijziging van het kern-DNA omdat wijziging van het mtDNA niet tot wijziging van essentiële kenmerken zou kunnen leiden. Zelfs als het mtDNA een basale cellulaire functie vervult, dan nog kan men verwachten dat modificatie van het mtDNA de identiteit van de toekomstige persoon verandert. We beargumenteren dat de ethische aanvaardbaarheid van NT voor mtDNA-ziekten niet zozeer afhangt van de vraag of de identiteit van een toekomstig persoon wordt veranderd (elke genetische modificatie zal dat waarschijnlijk doen), maar van de vraag of het recht van een kind op een open toekomst wordt geschonden. Dit hoeft niet het geval te zijn bij een klinische toepassing van NT voor mtDNA-ziekten. Mits veilig en effectief, is het gegeven dat NT voor mtDNA ziekten kiembaanmodificatie en mogelijk ook reproductief klonen behelst geen goede reden om het af te wijzen.

In hoofdstuk 8 onderzoeken we of het ethisch aanvaardbaar is om geslachtsselectie toe te voegen aan PGD en/of NT voor mtDNA ziekten. Een recente wetenschappelijke studie heeft aangetoond dat NT uitvoerbaar is bij primaten. Door deze technische doorbraak lijkt het slechts een kwestie van tijd voordat een eerste toepassing bij de mens overwogen wordt. De mogelijke aan toepassing van NT verbonden gezondheidsrisico's zijn echter een belangrijk punt van aandacht. Men verwacht dat het moeilijk te voorkomen zal zijn dat tijdens de transplantatie kleine hoeveelheden aangedane mitochondriën meekomen met de kern of voorkernen van de eicel van de wensmoeder. Ook al valt niet te verwachten dat dit tot ziektesymptomen in het resulterende kind leidt, dan nog kan het tot ziektesymptomen in een volgende generatie leiden. Dit is ook het geval wanneer bij PGD een embryo met een lage mutant load wordt teruggeplaatst. Door het mechanisme van de genetische flessenhals kan een lage mutant load in de tweede generatie (het kind geboren door middel van PGD) stijgen tot een hogere mutant load in de derde generatie (de kleinkinderen van het paar). Aangezien de mitochondriën via de moeder worden geërfd, zal bij mannelijk nageslacht de mutatie niet verder doorgegeven worden. Dit leidt tot de vraag of het voorkómen van transgenerationele gezondheidsrisico's een ethisch aanvaardbare reden is voor geslachtsselectie.

Geslachtsselectie kan plaatsvinden voor en na de conceptie. Bij preconceptionele geslachtsselectie (dus voor de bevruchting) worden de X-dragende en Y-dragende 
zaadcellen van elkaar gescheiden. Vervolgens wordt het Y-dragende sperma gebruikt voor de bevruchting. Bij postconceptionele geslachtsselectie worden embryo's tot stand gebracht door middel van IVF. Vervolgens worden alleen mannelijke embryo's gebruikt voor de totstandkoming van een zwangerschap.

We betogen dat geslachtsselectie ter preventie van transgenerationele gezondheidsrisico's in principe ethisch verdedigbaar is. Ook al staat de gezondheid van het toekomstig kind niet direct op het spel (waarmee geslachtsselectie traditioneel gesproken gerechtvaardigd wordt), de reden om voor een jongetje te kiezen heeft wel te maken met de gezondheid van het nageslacht. Een belangrijk punt van aandacht is echter de proportionaliteit: staan de materiële en immateriële kosten nog in redelijke verhouding tot het doel (het voorkomen van transgenerationele gezondheidsrisico's)? We beargumenteren dat het preferentieel terugplaatsen van mannelijke embryo's ethisch te verantwoorden is zolang PGD toch al wordt uitgevoerd. Het voor dit doel speciaal opstarten van een IVF/PGD-cyclus vinden we disproportioneel. In theorie heeft preconceptionele geslachtsselectie de voorkeur: het is minder invasief, minder duur en er worden geen embryo's weggegooid. In de praktijk blijkt geslachtsselectie in dit stadium echter feilbaar te zijn, met name wanneer Y-dragend sperma geselecteerd wordt. Niettemin zou preconceptionele geslachtsselectie een ethisch verantwoorde techniek kunnen zijn om de kans op mannelijk nageslacht te verhogen.

In hoofdstuk 9 wordt teruggeblikt op de belangrijkste resultaten, conclusies en beperkingen van dit proefschrift. Ook kijkt het vooruit naar belangrijke vragen voor toekomstig onderzoek. We concluderen dat alle strategieën, met de uitzondering van OT, potentieel aanvaardbare reproductieve mogelijkheden bieden voor dragers van mitochondriële mutaties. Aangezien en zolang OT ongeschikt is als een reproductieve optie voor dragers van mtDNA-ziekten en niet bewezen veilig, is het aanbieden van OT niet aan de orde. Voor prenatale diagnostiek, PGD en NT voor mtDNA-ziekten geldt dat geen van deze technieken onvoorwaardelijk acceptabel is: alle technieken zijn gebonden aan belangrijke voorwaarden en zorgvuldigheidsvereisten. Bij prenatale diagnostiek en PGD zijn er aanvullende criteria voor een verantwoord gebruik in de kliniek, voor NT is verder onderzoek in het laboratorium vereist voordat een klinische toepassing overwogen kan worden. Over de ethiek van een eerste toepassing van NT bij de mens is een nadere discussie noodzakelijk.

Dat mensen met een kinderwens zich tot een van de in dit proefschrift besproken technieken moeten wenden is in zekere zin tragisch, omdat alle reproductieve strategieën gepaard gaan moet moeilijke beslissingen en morele knelpunten. Zolang niet één van de strategieën duidelijk boven de andere uitsteekt (en ook moreel 
gesproken duidelijk te verkiezen is) is het redelijk om paren zelf de voor- en nadelen van de verschillende reproductieve strategieën te laten wegen. Het is belangrijk dat zij een beslissing kunnen nemen die te rijmen valt met hun eigen waarden en opvattingen, met hoe zij in het leven staan. Dat is hier natuurlijk niet de enige relevante overweging: het morele minimum zoals verdedigd in dit proefschrift luidt dat een hoog risico op ernstige schade voor het toekomstig kind voorkomen dient te worden, aangevuld met de inspanningsverplichting de risico's van een interventie zoveel als redelijkerwijs mogelijk te reduceren. 
Dankwoord 

Een proefschrift schrijven onder begeleiding van 'de twee Guido's' is een onvergetelijke ervaring. Twee heren, van dezelfde leeftijd, met dezelfde leerstoel. Toch zijn het heel verschillende personen die beide een andere rol in mijn promotietraject hebben gespeeld.

Prof. dr. G. de Wert, beste Guido, je scherpte, analytisch vermogen en enorme kennis van het vakgebied en daarbuiten zijn bewonderenswaardig. Als ik soms voor de $20^{\mathrm{e}}$ keer een tekst moest herzien had ik het weleens zwaar, maar ik heb er vooral van geleerd geen genoegen te nemen met eerste antwoorden en altijd nog een verbeteringsslag te maken. Ik hoop dat ik net zo'n goede onderzoeker als jij word! Daarnaast kijk ik met genoegen terug op onze leuke gesprekken en onze reizen naar de ESHRE congressen, waar ik verschillende onverwachte kwaliteiten van je leerde kennen, zoals je danstalent.

Prof. dr. G. Pennings, beste Guido, in onze eerste echte kennismaking in jouw mooie tuin nabij Leuven vroeg ik me enigszins wanhopig af of ik de gesprekken tussen jou en Guido ooit zou kunnen volgen. Maar naarmate we meer bijeenkomsten hadden begin ik de mitochondriën en de discussies beter te begrijpen. De flair waarmee jij ethiek bedrijft is aanstekelijk en een beetje jaloers ben ik wel op het gemak waarmee jij artikelen lijkt te schrijven.

Dr. W.J. Dondorp, beste Wybo, na ongeveer een jaar stapte jij van de Gezondheidsraad over naar onze afdeling en werd je mijn copromotor. Met de twee Guido's en jou erbij kon mijn project bijna niet meer mislukken. Naast je grote eruditie ken ik weinig mensen die zo vriendelijk doch constructief feedback kunnen geven. Dank daarvoor! Wat een luxe om als promovendus met drie van zulke heren zo uitgebreid over ethiek te kunnen praten, ik zal het zeker missen!

Dr. H.J. Smeets, beste Bert, als projectleider van MITOCIRCLE voegde je ook een workpackage Ethics aan het project toe. Deze inbedding van mijn promotieonderzoek in een internationaal consortium bood me een uitgelezen mogelijkheid om goede contacten te onderhouden met zowel het lab als de kliniek, en dat nog wel in een aantal Europese landen. Verschillende malen heb je me heel geduldig voorgetekend hoe de mitochondriën er uit zien, en hoe de overerving, de bottleneck en alle andere mechanismen werken. Dank voor het me wegwijs maken in de mitochondriële genetica!

Dr. C.E.M. de Die-Smulders, beste Christine, jij hebt me wegwijs gemaakt in de PGD en specifiek in PGD voor mitochondriële mutaties. Met je grote ervaring als zowel clinicus als onderzoeker wist je in onze discussies en artikelen ervoor te zorgen dat ook de klinische aspecten goed aan het voetlicht kwamen en we niet in theoretische spitsvondigheden verzandden. De gastvrijheid waarmee je me op congressen bij de afdeling klinische genetica inkaderde heb ik zeer op prijs gesteld. 
Geachte leden van de beoordelingscommissie, prof. dr. J. Geraedts, prof. dr. I.D. de Beaufort, prof. dr. ir. W. Bijker, prof. dr. J.L.H. Evers en prof. dr. J. Poulton, hartelijk dank voor het beoordelen van mijn manuscript. Prof. dr. J. Geraedts, beste Joep, gedurende het project hebben we regelmatig gesproken over PGD, meestal in de PGD-werkgroep. Dank dat je voorzitter wilde zijn van mijn beoordelingscommissie. Prof. dr. J.L.H. Evers, beste Hans, graag wil ik jou en de andere leden van de PGDwerkgroep in het azM bedanken, ik heb veel van jullie geleerd tijdens de maandelijkse besprekingen.

Prof. dr. J. Poulton, dear Jo, it was an honor to co-chair the ENMC meeting with you and thank you for your willingness to take part in the assessment committee and to travel to Maastricht for the defence.

During the research project I have had interviews with many patients, policy-makers and health care professionals in a number of European countries. I am very grateful for their time, their openness and their willingness to share their experiences with me. Prof. dr. P.F. Chinnery, dear Patrick, thank you for your willingness to have an ethicist in your laboratory at the Mitochondrial Research Group for a month. It was an eye-opener for me to spend some time with researchers at the bench.

De afdeling Health, Ethics \& Society was een vruchtbare plek om onderzoek te doen. Veel collega's hebben een waardevolle bijdrage geleverd, in discussies tijdens refereermiddagen, congressen, de lunch, bij het koffiezetapparaat of in café Thembi. Een paar mensen noem ik graag bij naam.

Dr. A. Krumeich, beste Anja, zonder jou had ik het empirisch onderzoek niet kunnen doen. Als er iemand expert op het gebied van kwalitatief onderzoek is dan ben jij het wel en zodoende ben ik je dankbaar dat je me wegwijs hebt gemaakt in de ins en outs van kwalitatief onderzoek. Prof. dr. R. Vos, beste Rein, dank voor het delen en overbrengen van je zeer brede kennis tijdens de ZTC-bijeenkomsten. Prof. dr. G. Widdershoven, beste Guy, dank dat je me meenam naar de onvergetelijke OxfordMount Sinai bijeenkomsten in New York en Oxford. Angelique, dank voor je hulp met het tijdig verzenden van de brieven voor de promotie.

Promoveren is soms een taai en eenzaam proces maar met collega-promovendi als Halime, Vivian, Bart, Josy, Patricia, Erik, Christi en Elleke sta je er bijna nooit alleen voor. Met name mijn kamergenoot Erik wil ik bedanken voor de ontzettend leuke tijd in Maastricht. Wat was je een fijne kamergenoot! Stiekem heb ik door jou ook veel meegepikt van jouw vakgebied, de Science\&Technology Studies, waarvoor dank! Christi en Elleke, met Erik erbij hebben we bijna alle biertjes van café Thembi geproefd en daarbij passeerden werkelijk alle onderwerpen de revue. Erik en ik zijn nu gepromoveerd, op naar jullie promoties! 
Ik heb een inspirerend nieuw onderkomen gevonden in de afdeling Medische Ethiek van het Julius Centrum van het UMC Utrecht. Prof. dr. J.J.M. van Delden, beste Hans, ik ben onzettend blij dat je me vorig jaar hebt aangenomen in het vertrouwen dat ik mijn proefschrift snel zou afronden en geleidelijk richting de research ethics zou schuiven. Dank voor het vertrouwen en de kansen en mogelijkheden die je biedt. Rieke, je schreef het al in jouw dankwoord: tien jaar geleden met elkaar in de collegebanken in Leiden, nu kamergenoten in het UMC. Ik hoop nog lang vriendin en collega van je te zijn! Beste Jonneke en Ghislaine, ook in jullie heb ik hele fijne nieuwe collega's gevonden. Nu jullie in de kamer naast ons zitten is er een heus 'ethiekblokje' in het Julius.

Mijn basis in de medische ethiek ligt in Leiden. Martine, jij was degene die in Leiden opperde dat ik maar eens een keuzevak medische ethiek moest gaan volgen. Moet je zien wat ervan gekomen is! Dank dat je me op dit spoor hebt gebracht en wat leuk dat je meegeschreven hebt aan het artikel over de interviews. Op naar jouw promotie! Bij mijn voormalige collega's in de afdeling Ethiek \& Recht van de gezondheidszorg van het LUMC, prof. dr. mr. Dick Engberts, dr. Dorothea Touwen en drs. Mirjam Houtlosser, heb ik mijn eerste vakken medische ethiek gevolgd, gewerkt als student-assistent en vervolgens als docent medische ethiek. Ook jullie wil ik bedanken dat jullie me vers uit de collegebanken hebben aangenomen als docent.

Lieve RISK clubgenootjes, het moet toch eens zwart op wit gezegd worden: wat zijn we een leuke club!

Lieve Shirin, het is elke keer weer leuk om met jou onder het genot van een wijntje werkelijk alles de revue te laten passeren. Binnenkort jouw promotiefeest!

Lieve Arjan en Fabienne (voor mij eigenlijk Willie), ik voel me helemaal sterk met jullie als paranimfen. Er kan op 18 juni niks meer mis gaan: krijg ik maagklachten dan wend ik me tot Arjan, voor alle vragen over genetica verwijs ik door naar Fabienne.

Arjan en Reinier, wat ben ik trots dat ik twee van zulke lieve grote broers heb. En gelukkig hebben jullie leuke vrouwen uitgekozen!

Lieve pap en mam, als er twee mensen zijn die onvoorwaardelijk voor me klaar staan, al mijn verhalen aanhoren en oprecht geïnteresseerd zijn in alles wat ik doe, dan zijn jullie het wel. Dank!

Lieve Barbara, een paar weken na de start van mijn promotietraject leerde ik je kennen. Al die jaren had jij een rotsvast vertrouwen in een goede afloop. Dit boek is nu af, maar ik hoop met jou aan mijn zij nog veel meer te schrijven! 



\section{Curriculum Vitae}



Annelien Bredenoord was born on August 1, 1979 in Utrecht, the Netherlands. In 1997 she graduated from College Blaucapel in Utrecht. Subsequently she studied Theology and Political Science at Leiden University. During her studies she worked as a teaching assistant at Leiden University Medical Center (LUMC), department of Ethics \& Law in Health Care and as a political assistant for D66-Leiden (social-liberal political party). During spring 2002 she was an intern at the scientific office of D66 in The Hague. She obtained her Master of Art degree in Theology in 2003 and in Political Science in 2006.

From 2003 until 2005, she was a lecturer in Medical Ethics at Leiden University Medical Center. In 2005 she moved to Maastricht University, department of Health, Ethics \& Society and research institute GROW, to start the research presented in this thesis under the supervision of prof. dr. G. de Wert, prof. dr. G. Pennings and dr. W.J. Dondorp. Her project was based on the Workpackage Ethics of the EU $6^{\text {th }}$ Framework Program MITOCIRCLE: "Mitochondrial diseases: from bedside to genome to bedside" (project leader dr. H.J. Smeets). She was member of the PGD ethics committee of Maastricht University Medical Center, taught ethics at the Medical School and supervised the NOMET-course, the national course for members of Research Ethics Committees. In September 2008 she obtained a grant of the Ter Meulen Fund, Royal Netherlands Academy of Arts and Sciences, to pay a study visit to the Mitochondrial Research Group of the University of Newcastle-upon-Tyne.

As of October 2008, she is employed as an assistant professor of medical ethics at the Julius Center of the University Medical Center Utrecht. She teaches ethics at the Medical School and is a member of the Consultation Committee of Reproductive Medicine and the Research Ethics Committee of UMC Utrecht. In her current ZonMW-funded research project, she examines whether individual genetic data should be disclosed to research participants in genetics/genomics research, and if so, which results, and by whom. In her spare time, Annelien has been member and chair of route66 and contributed to the D66 election program 2010. 